\title{
Translational research on spinal cord injury and cell- based therapies : a focus on pain and sensorimotor disturbances
}

Citation for published version (APA):

van Gorp, S. F. J. (2014). Translational research on spinal cord injury and cell-based therapies : a focus on pain and sensorimotor disturbances. [Doctoral Thesis, Maastricht University]. BOXPress. https://doi.org/10.26481/dis.20140314sg

Document status and date:

Published: 01/01/2014

DOI:

10.26481/dis.20140314sg

Document Version:

Publisher's PDF, also known as Version of record

Please check the document version of this publication:

- A submitted manuscript is the version of the article upon submission and before peer-review. There can be important differences between the submitted version and the official published version of record.

People interested in the research are advised to contact the author for the final version of the publication, or visit the DOI to the publisher's website.

- The final author version and the galley proof are versions of the publication after peer review.

- The final published version features the final layout of the paper including the volume, issue and page numbers.

Link to publication

\footnotetext{
General rights rights.

- You may freely distribute the URL identifying the publication in the public portal. please follow below link for the End User Agreement:

www.umlib.nl/taverne-license

Take down policy

If you believe that this document breaches copyright please contact us at:

repository@maastrichtuniversity.nl

providing details and we will investigate your claim.
}

Copyright and moral rights for the publications made accessible in the public portal are retained by the authors and/or other copyright owners and it is a condition of accessing publications that users recognise and abide by the legal requirements associated with these

- Users may download and print one copy of any publication from the public portal for the purpose of private study or research.

- You may not further distribute the material or use it for any profit-making activity or commercial gain

If the publication is distributed under the terms of Article $25 \mathrm{fa}$ of the Dutch Copyright Act, indicated by the "Taverne" license above, 


\title{
Translational research on spinal cord injury and cell-based therapies; a focus on pain and sensorimotor disturbances
}

\author{
PROEFSCHRIFT \\ ter verkrijging van de graad van doctor aan de Universiteit Maastricht, op gezag van de Rector \\ Magnificus, Prof dr. Luc Soete, volgens het besluit van het College van Decanen, in het openbaar te \\ verdedigen op vrijdag 14 maart 2014 om 10.00 uur door \\ Sebastiaan Franciscus Johannes van Gorp
}




\section{Promotores}

Prof. Dr. E.A.J. Joosten

Prof. Dr. M. van Kleef

\section{Copromotores}

Dr. J. Patijn

Dr. R. Deumens (Université Catholique Louvain, België)

\section{Beoordelingscommissie}

Prof. dr. R.J.E.M. Smeets (voorzitter)

Prof. dr. M. de Kock (Université Catholique Louvain, België)

Prof. dr. B.W.W. Kramer

Prof. dr. Y. Temel

Dr. J. van Zundert (Ziekenhuis Oost-Limburg, België) 


\section{Content - Chapters}

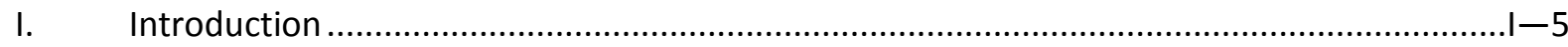

II. Exploring the causes of the large variety in reported pain prevalences in spinal cord injury

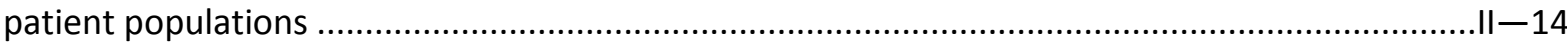

III. Spinally- and supraspinally-mediated pain-like response thresholds in the thoracic spinal cord contused rat; assessment and interpretation $. .111-38$

IV. Backwards locomotion on a rotating rod; a highly sensitive test to evaluate thoracic spinal cord contusion injury in rats? IV-48

V. Evaluation of olfactory ensheathing cell transplantation for spinal cord injury therapy in a rat hemisection model . $\mathrm{V}-60$

VI. Evaluation of human neural stem cell transplantation for spinal cord injury therapy in a rat lumbar injury model $\mathrm{VI}-70$

VII. Tumorigenesis safety study of human fetal neural precursor cells in $\mathrm{SCl}$ rats VII-106

VIII. Immunosuppression for human cell-based therapies in immunocompe-tent rats ...... VIII-134

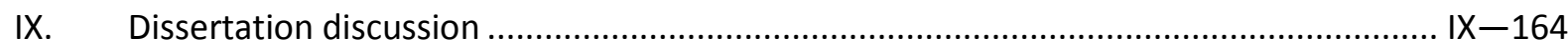

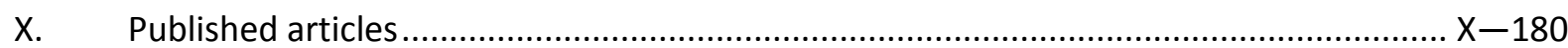

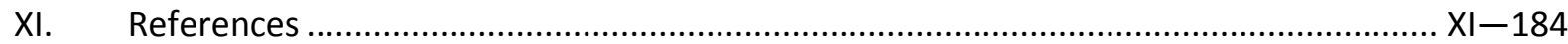

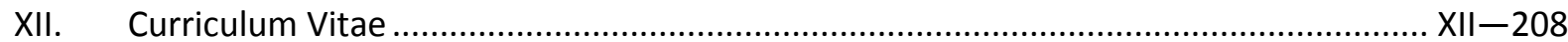

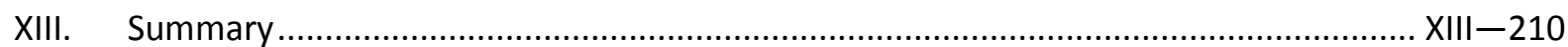

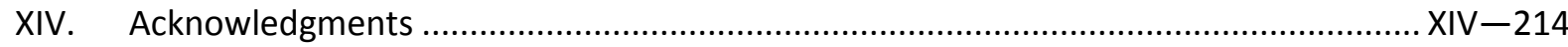




\section{Personal foreword}

I became interested in Neuroscience and in particular Spinal Cord Injury (SCl) research during the first years of my medical study at the University of Maastricht and I was further stimulated during my internship at the department of Pain Management and Anesthesiology. From this point onwards it became clear to me that chronic neuropathic pain is a major issue for many $\mathrm{SCl}$ patients. Pain, however, is clearly not the only problem after $\mathrm{SCl}$, and multiple sensory and motor modalities are usually altered by the injury (e.g. walking, spasticity and/ or the perception of heat and/or touch). Unfortunately, the gross similarity between all modalities stops here. While pain, for example, is marked by a relative over-activation in the nociceptive/sensory system, the motor system is often troubled by inactivity. Any $\mathrm{SCl}$ treatment strategy will, therefore, face a major challenge due to this dualistic aspect, which is even more difficult to tackle considering that the nociceptive/sensory system and motor system are anatomically intertwined. What is clear is that one should not investigate the effect of the treatment at hand on one sole modality while ignoring the other. The above explains why I choose the main theme of this thesis to be $\mathrm{SCl}$ treatment, keeping a special focus on SCl-related pain, but not letting non-pain related consequences of $\mathrm{SCl}$ get out of sight. 


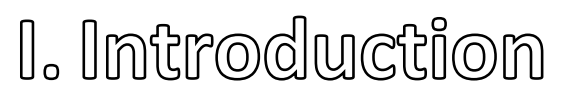




\section{Dissertation introduction}

The incidence of $\mathrm{SCl}$ is relatively low, with incidence rates from 1 to 8 per 100,000 and prevalence rates up to 80 per 100,000 (Wyndaele 2006). Due to the fact that most SCls have a traumatic cause related to sports, car/sports accidents, or violence, the patient population with new SCls is relatively young. Fortunately, modern medicine enables most SCl patients to have long life expectancies. The ancient Greek physician Hippocrates ( 400 B.C.), founder of Western medicine, deemed most SCI patients were destined to die, and it was only by the mid- $20^{\text {th }}$ century, a time at which $\mathrm{SCl}$ care revolutionized, that this view changed. The improvement in SCl care was, ironically, mostly thanks to World War II, as army hospitals at the time were one of the first to establish $\mathrm{SCl}$ care units (encouraged by physician Donald Munro; 1898-1978). The dogma that most SCls are "an ailment not to be treated", which can even be traced back about 5000 years to the writings of an Egyptian physician (most likely Imhotep), was finally broken. This change was also fueled by recent medical discoveries, the most important of which was the discovery of penicillin (Alexander Fleming; 18811955), resulting in the fact that infections like pyelitis were no longer a major cause of death in SCI patients. In current times, most SCl persons, at least in highly developed Western world countries, achieve a fairly independent societal integration and long-term survival with nearly normal life expectancies. This sets the scene for the $\mathrm{SCl}$ research community, as the secondary complications of $\mathrm{SCl}$, like pain and spasticity, are becoming more and more relevant issues for $\mathrm{SCl}$ patients. For instance, $\mathrm{SCl}$ patients with significant pain are generally willing to trade a chance of recovery or loss of certain physiologic functions (sexual, bowel-bladder control) for the relief of pain (Nepomuceno 1979). In addition, the intensive and chronic aspects of SCl care is making this debilitating condition expensive, yielding high costs for highly developed western societies (estimated to approach \$7 billion each year in the United States) and, at the same time, causing it to remain unavailable in developing countries (Burns 2012, McDonald 2002).

\section{Current SCl treatment options are limited}

Modern treatment of $\mathrm{SCl}$ can be divided into two phases; one timed to the immediate/acute phase and the other timed to the post-acute phase. In the acute phase, the treatment aims at prevention of further damage to the spinal cord. This is the reason why trauma patients are immobilized (e.g. by a backboard and rigid collar) as soon as possible (Bernhard 2005). Next, the spine is stabilized either by surgical intervention, a rigid brace, and/or axial traction. In the early 1990's a steroid antiinflammatory drug, methylprednisolone (MP), was systematically added to the acute phase treatment as it was believed to suppress the deteriorating effects of immune cells near the site of injury and reduce the damage these cells inflict on the spinal cord. The efficacy of MP treatment is, 
however, debatable (Hurlbert 2000). The three large randomized controlled trials with MP in SCI patients show only very modest effects, if any, on gain of neurological status (Bracken 1985, Bracken 1993, Bracken 1998). Furthermore, this therapeutic effect does not seem to result in any functional improvement, raising the question whether the risks for adverse effects due to high dose MP should be taken at all (Matsumoto 2001). In the Netherlands, MP is not recommended for the treatment of SCI (Vandertop 1998). Treatment timed to the post-acute phase, is initiated by a rehabilitation team through physical, communicational, and emotional support as well as education. In a follow-up, this multidisciplinary treatment is further extended through additional vocational and recreational therapy, all together providing opportunities for socialization and normal lifestyles. Secondary complications of $\mathrm{SCl}$, such as a variety of pain problems, spasticity, bladder/bowel control function, and autonomic dysreflexia are also intensively addressed in the post-acute phase, but treatments are few and if available often with poor efficacy. For instance, currently available drug treatments for persistent pain and spasticity after $\mathrm{SCl}$ have limited therapeutic effect and are often badly tolerated (Teasell 2010). In conclusion, current SCI treatments can, at best, offer a partial prevention of further loss of function after the initial trauma and a limited improvement in secondary complications, but major health problems remain.

\section{SCl pathophysiology}

A great deal of $\mathrm{SCl}$ research focuses on the question whether normal function of an injured spinal cord can be restored. Unfortunately, the development of $\mathrm{SCl}$ regenerative treatments has proven to be extremely difficult. The adult mammalian spinal cord shows an intrinsic poor regenerative capacity. $\mathrm{SCl}$ also results in the formation of scar tissue and cavities (cysts/syringes) which are impediments for regeneration of severed axons. Since countless fibers, which project to a wide variety of structures, run intertwined through the spinal cord, reconnecting and retraining of neural circuits is an extremely difficult task (Deumens 2005). A further complicating factor in the design of $\mathrm{SCl}$ treatments is the fact that loss of spinal cord tissue and the formation of cysts and scar tissue is not merely the result of the primary injury, but is also due to a series of secondary pathophysiological processes which occur from minutes up to months after injury. These processes are triggered by the hemorrhages and swelling after the vascular damage, which is, together with necrosis and disruption of axons, one of the components of the primary injury. Secondary damage is the consequence of ischemia, lack of nutrients, oxidative stress, excitotoxicity (cell death due to an excessive excitatory drive due to massive release of the neurotransmitter glutamate), electrolyte imbalance of intra- vs. extracellular compartments, and inflammatory reactions regulated by both resident immune cells and leukocytes infiltrating the spinal cord parenchyma via a breach in the blood-brain barrier (Alexander 2009, Hagg 2006). In response to the injury, spinal cord support cells 
shield off the injury site by creating an impenetrable barrier that is mostly made up of extracellular matrix molecules like collagen-based proteoglycans intermingled with reactive astrocytes and microglial cells; i.e. the glial scar (Bruce 2000, Fawcett 1999, Gris 2007, Silver 2004, Thuret 2006). The scar inhibits a further expansion of the secondary damage zone and eventually forms the lining of the cavity/-ies that form at the lesion epicenter (Faulkner 2004). Although restriction of further expansion of the lesion is basically an important protective mechanism, the cavities and scars, at the same time represent a mechanical and chemical barrier for axonal growth and, thus, prevent regeneration. Furthermore, while poorly understood, progressive enlargement of the cavity, a condition called (progressive) posttraumatic syringomyelia is known to be a fairly common additional complication (Brodbelt 2003). This condition forms a risk for further deterioration of patient, including the gradual onset/worsening of secondary complications, especially pain (Wang 1996).

\section{SCl pain: what does it entail and how does it work?}

Albeit still challenging it is generally clear what is needed for spinal cord repair; not only neuroprotection but also promoting regrowth of severed axons and replacement of lost cells followed by retraining of preserved or newly formed neural circuits. What is needed for treatment of persistent pain after $\mathrm{SCl}$ is, unfortunately, much less evident. This starts from a relatively poor understanding of the underlying pathophysiological mechanisms of chronic pain after SCl. Moreover, experimental findings obtained in animal studies are often difficult to translate the clinical arena, partially due limitations in systematically investigating pain in (rodent) animal models. The lack of verbal communication about perceived pain by animals makes analysis of pain even more difficult than in humans. In the experimental pain field the golden standard and most frequently used to test and assess pain in rodents involves measurement of evoked pain-like responses such as paw withdrawal following mechanical or thermal stimulation of the skin (Christensen 1996, Hao 1991). In this respect, below-level SCI pain (pain perceived/projected in areas below the level of injury) is considered to be reflected by reductions in withdrawal thresholds to hindpaw stimulation in animal models of contusion or hemisection of the mid/low thoracic spinal cord (Bennett 2000b, Christensen 1996, Hains 2006). With the use of this technique, many types of SCI models are characterized by reductions in the threshold of hindpaw withdrawal following mechanical or thermal stimulation, indicating the involvement of hyperexcitabile neuronal circuits (Hains 2005). Hyperexcitability can be due to several processes including the formation of new dorsal horn circuitries (Christensen 1997), imbalances in inhibitory systems of the spinal cord (Kim 2010), and other mechanisms (Gwak 2011a). From a clinical point of view it is known that a partial preservation of the large ascending spinothalamic tract (located in the ventrolateral spinal white matter) is related to hyperexcitability 
and, thus, required for an augmentation of evoked pain (Garcia-Larrea 2002). However, concerns are raised that paw withdrawal responses are not necessarily related to increased pain sensitization after $\mathrm{SCl}$, as such responses often do not coincide with pain behaviors requiring higher brain centers (e.g. expected escape behavior to painful contexts) and even occur in spinalized animals (Baastrup , Yezierski 2010). It has, therefore, been proposed that these responses more closely relate to the spastic syndrome (a collection of symptoms related to exaggerated spinal reflexes induced by CNS lesions). Only a few studies/research groups have yet used the more appropriate escape/avoidance behavior test as a measure of pain in animal models of SCI (Baastrup 2010b, Vierck 1995).

Animal studies have shown that sprouting of nerve fibers contributes to neuronal hyperexcitability, not only in the nociceptive system, but also in systems that relate to motor and autonomic functions, thereby triggering spasticity and autonomic dysreflexia (Brown 2012). In this perspective, the occurrence of inhibitory cues like scar tissue and cysts are not necessarily bad, as they would to some extent prevent neuronal hyperexcitability. On the other hand, nerve growth/regeneration of injured motor axons is desired for regaining motor functionality. It is vital to realize that the structural plasticity in nerve fibers, with the formation of new neuronal connections, creates a risk for hyperexcitability, also in the nociceptive/sensory system (Deumens 2008), and thus a potential increase of secondary complications (see figure I-1). With respect to central pain (pain caused by a lesion or disease of the central somatosensory nervous system; definition of the International Association for the Study of Pain; IASP), mechanisms of hyperexcitability seem to be at least involved in the hypersensitivity to stimuli (the evoked pain component). Whether the same concepts of hyperexcitability are also relevant for the spontaneous (non-evoked) pain component of central pain, is less evident. By definition, spontaneous pain is pain due to changes that are internal and selfcontained with respect to the somatosensory system (Bennett 2012). Then, spontaneous pain has no (apparent) trigger, shunting a role of hyperexcitability in this phenomenon. Alternatively, spontaneous pain might be related to a yet unknown 'trigger' or source. In support of this latter possibility, it was recently reported that $\mathrm{SCl}$ patients with an incomplete injury showed signs of neuronal hyperexcitability (heat evoked hyperpathia, dynamic mechanical allodynia), which often preceded the onset of spontaneous pain (Zeilig 2012). 


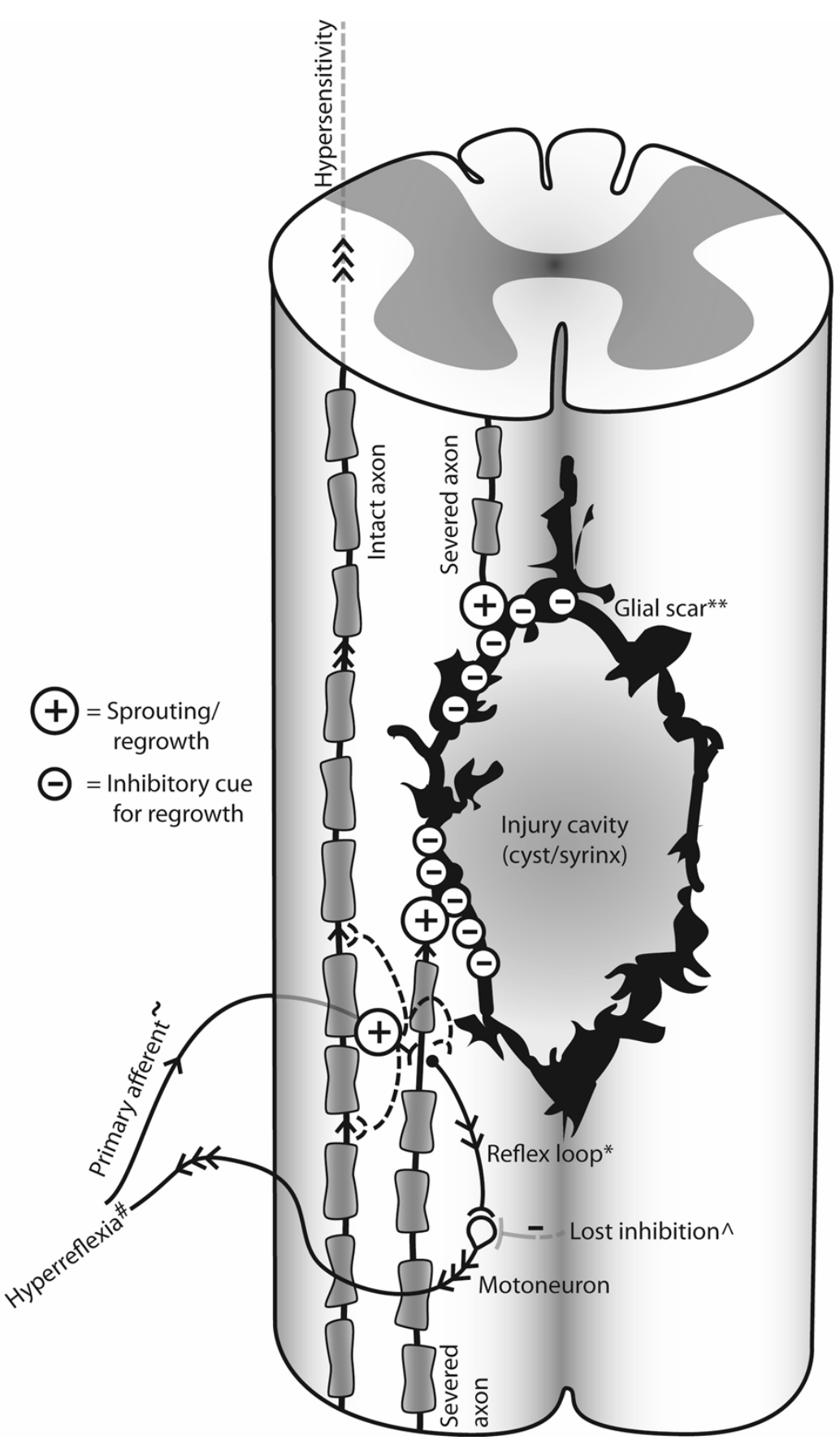

Figure 1-1: Abstract drawing of a section of an injured spinal cord, after formation of an injury cavity and glial scar**. Shown are severed axons which (endogenously) attempt sprouting/regrowth(+) after injury. However, the latter is inhibited(-), mainly due to the glial scar**. Also shown are the primary afferents $\sim$ which are also sprouting in response to the injury and gain increased connectivity to neurons projecting to supraspinal levels and interneurons of the reflex loop*, resulting in hypersensitivity and hyperreflexia", respectively. In addition, reflex loops (with variable complexity) typically lose inhibitory tone^ ${ }^{\wedge}$ wen located at/below the injury, causing reflexes to become easily activated, resulting in a variety of hyperreflexia"-related features (i.e. the spastic syndrome). 
Hence, processes of neuronal hyperexcitability, as yet identified in animal models, may also play a role in spontaneous pain, but the source of the spontaneous generator, or actual pain generator, remains to be identified. Some studies claim a role for the spinothalamic tract as residual function and activation of this tract seems to plays a role in spontaneous pain even with clinically complete SCls (Hari 2009, Wasner 2008). Others have emphasized that patients with complete interruption of the spinothalamic tract can also develop spontaneous pain, suggesting that the pain generator (and hyperexcitability) ought to reside present somewhere above the level of injury (Finnerup 2003a, Melzack 1978).

So far, studies investigating differences in characteristics between $\mathrm{SCl}$ patients with and without pain remain inconclusive, as studies typically report different findings (Finnerup 2012a). However, these differences in findings might be due to the distinct characteristics of studies, a fact that is also reflected in the high level of heterogeneity between SCI pain prevalence studies (Dijkers 2009). The causes for this heterogeneity might uncover strong determinants for pain after $\mathrm{SCl}$, but are yet poorly investigated.

\section{Advances in $\mathrm{SCl}$ treatment: what is needed?}

What is clear from the above is that, when considering treatments to promote regrowth and regeneration following $\mathrm{SCl}$, one should at least prevent the further induction of neuronal hyperexcitability which worsens secondary outcomes, like pain and the spastic syndrome. Already some time ago, it was found that injured axons of CNS neurons could grow into peripheral nerve grafts implanted into the injured spinal cord, and it was found that the CNS itself contained inhibitory signals for axonal growth (David 1981). Later, inhibitory signals/molecules present in the injury site and scar were identified and targeted; for the most studied ones being proteoglycans (chondroitin sulfate proteoglycans (McKeon 1995)) and molecules associated with myelin/myelin debris (Nogo, myelin associated glycoprotein, and oligodendrocyte myelin glycoprotein (Fournier 2001, Mueller 2005)). The balance within the CNS favoring inhibition over promotion of neurite outgrowth might be reversed by applying growth/neurotrophic factors to the injury site (e.g. Nerve growth factor (NGF), Glial derived neurotropic factor (GDNF), neurotrophin-3 (NT-3)) (Fortun 2009, Houweling 1998, Koelsch 2010). Furthermore, cell-based therapies can also provide beneficial effects through an array of mechanisms. For instance, immature astrocytes may be transplanted into the injured spinal cord as these cells are able to produce neurotrophic factors, scavenge excess/excitotoxic neurotransmitters, and limit immune responses after $\mathrm{SCl}$ (Krenz 2000, Okada 2006, Rothstein 1996). Treatments based on the above mentioned application of neurotrophic factors, however, carry the risk of worsening secondary outcomes of $\mathrm{SCl}$ by unspecific increase in 
neuronal plasticity, which may lead to hyperexcitability. Indeed, it has been shown that, at least in animals, treatment with Nerve growth factor or transplantation of (certain types of) astrocytes can lead to increased neuronal excitability (Davies 2008, Gwak 2003, Hofstetter 2005). Besides these risks, the transplantation of immature, neurite-outgrowth promoting astrocytes into the lesion gap of $\mathrm{SCl}$ rats has in some cases even been found to fail in stimulating regrowth of severed axons or functional recovery (Deumens 2006b, Joosten 2004). Hence, selection of the appropriate cells for transplantation may be of utmost importance as cell-based therapies are often regarded as one of the most promising treatments for $\mathrm{SCl}$ considering that grafted cells can be used to both stimulate regrowth of severed axons and replace lost cells.

\section{Olfactory ensheathing cells}

One cell-based therapy of particular interest in the field of $\mathrm{SCl}$ and repair is the transplantation of olfactory ensheathing cells (OEC). OEC are derived from the olfactory system as these cells reside in the olfactory bulb, olfactory nerve, and even in the olfactory mucosa, supporting the growth of axons all the way from the nasal epithelium into the CNS throughout the life of mammals. The extraordinary neurite outgrowth promoting capacities of these glial-like cells (Deumens 2006c), and the fact that they can be used in an autologous transplantation strategy while requiring minimal pregrafting in vitro manipulations make these cells a prime candidate for $\mathrm{SCl}$ repair. Another major characteristic of (transplanted) OEC is their ability to intermingle with reactive astrocytes and migrate through scar tissue, thereby transforming the neurite outgrowth inhibitory scar into a permissive area (Raisman 2007). Moreover, OEC form channel-like structures that serve as a substrate for regrowth of severed central axons through the growth restrictive injury site, which then may result (after remyelination) in functional improvement (Li 1997, Lopez-Vales 2006, RamonCueto 2000). In addition, no signs of neuronal hyperexcitability have been noted below the level of injury after OEC treatment in rodent models (Lopez-Vales 2007, Ramon-Cueto 2000). OEC specifically acquired from the lamina propria (instead of the olfactory bulb), however, have shown to induce autotomy behavior in areas below the level of injury, which suggests the occurrence of pain (Richter 2005). The use of such interventions in human patients is, however, still made difficult as human injuries are often characterized by large cavities which are mainly absent in standard animal models of SCl (Bunge 1993). A further issue is potential adverse effects of OEC transplantation. In an experimental model where OEC were injected into the injury site in rodent SCI models neuronal hyperexcitability has been noted at the level of injury (Bretzner 2010), an effect that might be due to the release of neurotrophic factors by OEC (Woodhall 2001). Unfortunately, OEC transplantation in repair of experimental spinal lesions with large cativities was up till now not very successful. The use of poly $(\mathrm{D}, \mathrm{L})$-lactide matrices carrying aligned OEC on their surface into large spinal lesion cavities 
(dorsal hemisection T13-L1) of adult rats has only marginal effects on severed axons (i.e. corticospinal axons were found more closely to the lesion site, but not not cross the lesion). This lack of axon regeneration could, however, be related to the characteristics of the scaffold material; the break-down products of poly $(\mathrm{D}, \mathrm{L})$-lactide are acidic which may be detrimental for good integration of the scaffold within the spinal cord lesion; also the matrices used only supported seeding OEC on the matrix surface leaving the grafted cells directly exposed to the toxic environment of the injured spinal cord (Deumens 2006a).

\section{(Human) neural stem cells}

Another promising cell-based therapy for repair of the injured spinal cord involves (human) neural stem cells. These cells have the potential to provide cell-replacement for both neurons and glia. However, it has been -and still can be- a main problem that neural stem cells tend to mostly differentiate into astrocytes, which, in some cases, can support neuronal hyperexcitability (Hofstetter 2005, Martino 2006, Setoguchi 2004). Neuronal and oligodendroglia differentiation would certainly lead to better therapeutic effects of neural stem cell treatment (Abematsu 2010, Cummings 2005, Keirstead 2005). Neurons derived from neural stem cells are able to fully integrate into the host circuitry and their oligodendrocytes effectively remyelinate axons (Englund 2002, Keirstead 2005). Human fetal spinal cord derived neural stem cells (HSSC) grown in a monolayer, have shown to give rise to neurons, astrocytes, and oligodendrocytes in a rodent $\mathrm{SCl}$ model (Yan 2007). Furthermore, these HSSC were shown to be able to reduce spasticity (and improve locomotor function) after intraspinal grafting in a rodent $\mathrm{SCl}$ model of spasticity, which was attributed to their differentiation in inhibitory ( $\gamma$-aminobutyric acid-ergic (GABA) and glycinergic neurons (Cizkova 2007). Lack of inhibition might be an important mechanism for neuronal hyperexcitability underlying pain and spasticity after SCl (Gwak 2011b). Furthermore, by improving the inhibitory tone in the dorsal horn it was shown that also injury-induced aberrant sprouting of sensory nerves was reduced in a rodent SCI model (Liu 2004). Hence, transplantation of HSSC holds promise to induce functional recovery and reduce/prevent secondary complications after $\mathrm{SCl}$. One concern with the use of stem cells, however, is that they might continue to proliferate and fail to differentiate, thereby forming tumors (Amariglio 2009). Another concern with the assessment of the efficacy of human stem cells in rodent $\mathrm{SCl}$ models is graft rejection. Successful translation of cell replacement therapies which utilize xenogeneic or allogeneic cell grafts depends on the design of safe and effective immunosuppression protocols which will permit long-term survival and maturation of grafted cells. Current clinical and experimental immunosuppression protocols typically use single or combined immunosuppressive drug regimen with drugs delivered orally, intraperitoneally, intravenously, or subcutaneously in a single daily dose or divided into multiple daily doses (Barraclough 2011, 
MacGregor 1995). While in human clinical settings a targeted plasma concentration of a variety of immunosuppressive drugs can be effectively achieved through drug dose titration, the accomplishment of a comparable consistency in targeted plasma levels in animal studies remains a major challenge. Identifying a consistent slow-release formula, such as liposomes, biodegradable microspheres, or biodegradable pellets, would aid tremendously as an effective, safe and simple method for immunosuppression in laboratory animals.

\section{Validity of functional outcome measures}

Furthermore a valuable preclinical assessment of cell-based therapies not only depends on adequate cell survival in laboratory animals, but, as with other therapeutic assessments, also on the validity of functional outcome measures in these experimental animals. Ideally, one would like to use tests which perfectly correlate with the situation in human SCl-patients and are validated in such a way, but serious limitations exist. First, the trauma causing the $\mathrm{SCl}$ in humans differs often, if not always, from experimental models as human $\mathrm{SCl}$ are highly variable, if not unique, among individual patients whereas animal models basically comprise standardized reproducible lesion types. Second, no pharmacological treatment has yet given consistent results in human $\mathrm{SCl}$ patients, despite several positive outcomes reported in experimental SCl-models. It is still remarkable that the few large randomized controlled trials of promising (pharmacological) treatments (judged on positive preclinical studies) all resulted in a negative clinical outcome (Tator 2006). Thus, apart from the inherent difficulty of developing an effective $\mathrm{SCl}$ treatment, the common ways of assessing therapy efficacy in $\mathrm{SCl}$ models does not optimally correspond with the clinical problem in human $\mathrm{SCl}$ patients. As mentioned above, widely used sensory/pain outcome measures in preclinical SCl models are heavily criticized for low translational value towards sensory/pain problems in human patients (Baastrup 2010b, Hofstetter 2003); it is hypothesized that paw withdrawal after SCl correspond better with the spastic syndrome. The spastic syndrome in humans basically consists of positive (i.e. gained, as opposed to lost) motor signs of the upper motor neuron syndrome, such as spasticity (the occurrence of velocity dependent reflexive increase in muscle tone upon passive muscle stretch), hyperreflexia (exaggeration of normal reflexes), spasms (involuntary muscle contractions, often due to some kind of stimulation), and clonus (automatic repetitions of reflex responses); negative signs are decreases in muscle tone, weakness, loss of voluntary control). The spastic syndrome, however, is typically deemed less prevalent in rodent models of SCl than in human SCI (Bennett 1999), although it is possible that the spastic syndrome is merely less evident in rodents instead of less prevalent (Baastrup 2010b, Bennett 1999). In human SCI patients, the spastic syndrome occurs when negative signs of the upper motor neuron syndrome are obvious; i.e. walking becomes a problem, however these 'spastic syndrome-related' paw withdrawal responses are seen in adequately walking 
rats (Christensen 1996). This difference between species could be a reason why symptoms of the spastic syndrome are overlooked in rodents, as the overall clinical image is very different (walking rats vs. paraplegic humans). It would, thus, be interesting to know whether the 'paw withdrawals spasms' also occur in the acute phase after $\mathrm{SCl}$ in experimental $\mathrm{SCl}$ pain models. During this phase motor function is still very poor and any motor activity would indeed be indicative for the onset of spasm-like reflexes. This could then be further evidenced by the use of animals with severe SCI, which are known to recover minimally and show poor voluntary movement, even during the postacute phase. This approach may allow studying if such spasm-like reflex behavior can remain for longer times after injury. As spasticity (one sign of the spastic syndrome) is defined as 'a velocitydependent increase to tonic muscle stretch', this aspect of the spastic syndrome might, next to 'paw withdrawal spasms', simply not have been adequately assessed yet in the experimental SCI-field (Baastrup 2010b). In this context, one might expect a decrease in escape behavior to stimuli causing paw withdrawals after $\mathrm{SCl}$ in more severely injured animals when compared to uninjured animals.

It is unmistakably clear that major interspecies differences exists with respect to the spastic syndrome and its interpretations as a discrepancy between positive and negative signs of the upper motor neuron syndrome seem obvious. Indeed, the spastic syndrome goes unnoticed in most animal models of SCl, while in the clinic the term 'spastic syndrome' is often used almost synonymously for the upper motor neuron syndrome (Ivanhoe 2004). The difference in extent of negative motor signs after $\mathrm{SCl}$ might be due to a high spinal autonomous motor function in animals, when compared to humans. Spinalized laboratory quadrupeds (like rodents) are renowned for their ability to show impressive walking patterns, as long as their weight is supported for most of the time (Grillner 1973). However, primates are likely to have lost this spinal autonomy of hindlimb locomotion (Eidelberg 1981). This poses a further problem on widely used functional motor outcome assessments in SCI models (mostly rodent models), as the sensitivity of such tests to detect reconnection of supraspinal structures with spinal targets may be low. Indeed, only relatively severe injuries achieve some chronic locomotion deficits, suggesting regenerative effects can be easily missed using such functional tests (Basso 1995, Basso 1996). Furthermore, although spinal autonomous locomotion patterns are important for rodent/quadruped SCl locomotion recovery, it is believed that primates depend more on spinal cord regeneration (Rosenzweig 2010). A serious re-evaluation of currently used functional tests is required and development of new methods to test behavioral recovery of sensory as well as motor outcome after $\mathrm{SCl}$ are urgently needed. 


\section{Objectives of research in this thesis are:}

1) Explore causes for the heterogeneity in prevalence rates, and find determinants for pain after $\mathrm{SCl}$,

2) Systematically explore tools (sensory and motor assays, and immunosuppression protocols) for the preclinical and translational evaluation of $\mathrm{SCl}$ treatments, and

3) Test the relevance and feasibility of candidate cell-based therapies (OEC and $\mathrm{HSSC}$ ) for SCI treatment in preclinical models.

\section{Outline and research questions of this thesis}

It is evident that $\mathrm{SCl}$ represents a multifaceted clinical challenge. Although improvements in health care have given $\mathrm{SCl}$ patients a good life expectancy and autonomic position in society, major health problems and complications remain. Chronic, poorly treatable pain still is one of the most severe among the various complications, and thus of high relevance when designing $\mathrm{SCl}$ treatments. Unfortunately, pain after $\mathrm{SCl}$ is poorly understood as predictors or mechanisms for pain syndromes after $\mathrm{SCl}$ remain obscure. Reports suggesting an association between patient characteristics (e.g. level or cause of injury) and the onset of pain after $\mathrm{SCl}$ vary, and thus remain inconclusive. On the other hand, the reported pain prevalence after $\mathrm{SCl}$ also varies tremendously, suggesting important differences between studies. Dissimilarities in study characteristics/methodology (and patient populations) can cause differences in the relationships found between patient characteristics and the occurrence of pain. To know which between-study differences result in differences in pain prevalence can further aid in the identification of those patient characteristics which relate most to pain after $\mathrm{SCl}$, and might help to isolate the mechanisms of pain after $\mathrm{SCl}$. Therefore, the first research question of this thesis is:

1. Is it possible to identify which study and/or population characteristics show a relationship with the differences in reported pain prevalence rates in $\mathrm{SCl}$ patient populations?

Chapter 2 explores which differences between studies are causing dissimilar pain prevalence outcomes. A systematic review of studies reporting pain prevalence in populations with $\mathrm{SCl}$ is performed and study and (sub-) population characteristics are compared using meta-analytic methods.

Development of $\mathrm{SCl}$ therapies requires preclinical research, which in turn relies largely on animal models, mostly rodents. Compared to in vitro models, animal models offer the advantage of 
assessing functional benefits of the tested therapy through behavioral tests. However, differences between animals and humans might be highly relevant when assessing $\mathrm{SCl}$ outcomes. The high biomechanical stability of a quadruple gait, compared to human bipedal walking, allows more locomotion control to be executed through spinal reflexes. These spinal reflexes are then difficult to distinguish from actual recovery of $\mathrm{SCl}$ (i.e. restoration of circuitry with supraspinal structures), destabilizing the value of animal models for $\mathrm{SCl}$ outcomes. This is not only relevant for locomotion assessments after $\mathrm{SCl}$, but also for pain tests. The cornerstone of pain assessments in animal models relies on evoked pain-like responses. Therefore, the second research question of this thesis is:

2. Can we differentiate between spinal reflex mediated functionality and supraspinally mediated functionality in rodent $\mathrm{SCl}$ models?

In Chapter $\mathbf{3}$ we explore the clinical relevance of paw withdrawal responses as observed in a widely used rat model of $\mathrm{SCl}$ and $\mathrm{SCl}$ pain: a thoracic spinal cord contusion injury, which is inflicted by a standardized weight-drop (Gruner 1992, Hains 2006). Furthermore, in Chapter 4 we test which behavioral assay reflects recovery of supraspinally mediated locomotion functionality. However, due to the unavailability of a proven effective $\mathrm{SCl}$ treatment for locomotion recovery in humans (a positive control), in Chapter 4 we investigate which behavioral test is most sensitive for locomotion changes related to different $\mathrm{SCl}$ severities.

Due to their regeneration promoting properties, some cell-based therapies like Olfactory Ensheathing Cells (OEC) and human Fetal Spinal Neural Stem Cells (HSSC) form a promising treatment for SCl. Olfactory Ensheathing Cells (OEC) and Human Fetal Spinal Neural Stem Cells (HSSC) are particularly interesting. OEC naturally promote axonal growth into the CNS (Ramon-Cueto 1998). Moreover, they can be used for autologous grafting, circumventing the need for immunosuppression (Munoz-Quiles 2009). These properties are useful, but SCl in humans generally results in the formation of cavities (syringes) in the spinal cord which also requires structural bridging (Bunge 1993). This problem might be overcome by the use of a multichannel, biomatrix scaffold which aligns with the severed projection axons of the host spinal cord to transfer OEC to the injured spinal cord. On the other hand, HSSC can give rise to new neurons and glia cells, and provide growth-promoting factors, providing both support and a new supply of neurons to the injured spinal cord.

3. Does transplantation of OEC or HSSC into the lesion site provide functional improvement in an experimental $\mathrm{SCl}$ model? 
In Chapter $\mathbf{5}$ we investigate whether OEC seeded into a multichannel, collagen scaffold can facilitate axonal regrowth and functional recovery across an cavity created at the thoracic level of the adult rat spinal cord (Christensen 1996). In this model, the cavity is created by unilateral removal of a $2 \mathrm{~mm}$ long section of the spinal cord. In Chapter 6 we inject HSSC into and around a spinal cavity formed after traumatic spinal cord compression. This model uses a 15 minute compression (35grams) of a lumbar segment (L3), just above the lumbar segments innervating the hindpaw/sciadic nerve. In both OEC and HSSC transplantation approaches we explore the therapeutic effects on motor function, sensory thresholds, and/or evoked paw withdrawal thresholds (below the level of injury).

Although HSSC can both provide support as well as replenish cells to the injured spinal cord, they pose a risk as they are not fully differentiated and can continue to proliferate, and therefore might form tumors in the host spinal cord (Amariglio 2009). Moreover, autologous transplantation of fetal HSSC is not possible, necessitating continuous immunosuppression of the host, especially after xenotransplantation (in laboratory animals). Therefore, the last research question of this thesis is:

4. Is long-term HSSC engraftment safe for clinical use and achievable with the use of slowrelease immunosuppressive drug formulas?

Therefore, we assessed the safety of HSSC-therapy for intraspinal grafting, principally regarding their application in future clinical trials. Specific guidelines for such safety assessments of cell-based therapies are lacking. In Chapter $\mathbf{7}$ we report on the results and the considerations of a HSSC safety study. Its design was the result of a dialog with the Food and Drug Authority (FDA; the national drug authority in the United States) and its outcome has resulted in the approval of a first clinical trial with these fetal HSSC. Chapter 8 describes an adequate regime for long-term immunosuppression when grafting HSSC in immunocompetent rats without the need for laborious immunosuppression protocols. The latter is based on the performance of different slow-release formulas in comparison to the regularly used (twice) daily injections of immunosuppressive drugs. Both pharmacokinetics and cell-survival are used as main outcome measures to assess the quality of the immunosuppression. 
0. Exploring the causes of tine Darge

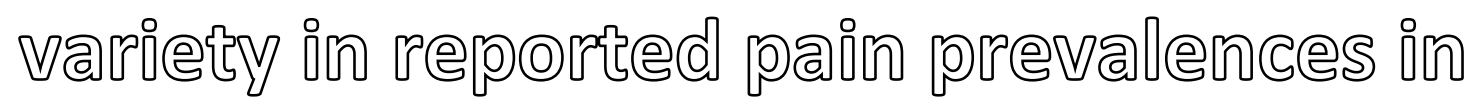

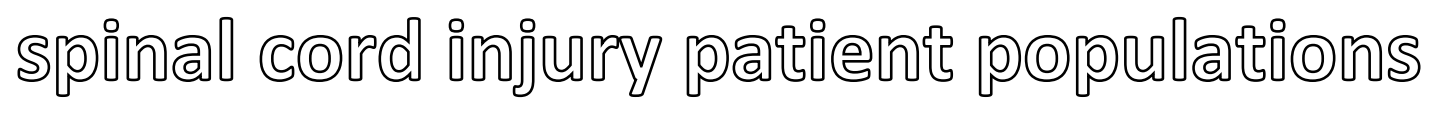




\title{
Pain prevalences and their large heterogeneity after spinal cord injury: a systematic review
}

Sebastiaan van Gorp; Alfons G. Kessels; Elbert A. Joosten; Maarten van Kleef; Jacob Patijn

\begin{abstract}
Pain prevalence studies are important as they illustrate the magnitude of pain problems in a certain patient population, such as patients living with a spinal cord injury ( $\mathrm{SCl}$ ). Strikingly, reported pain prevalence rates in $\mathrm{SCl}$ patients are found to vary greatly, while determinants for the differences between pain prevalence reports remain unclear. We here aim to identify determinants for the differences (heterogeneity) in pain prevalence reports through a systematic review of all $\mathrm{SCl}$ pain prevalence reporting studies. Literature search using MEDLINE, Cumulative Index to Nursing and Allied Health Literature (CINAHL), ISI Web of Knowledge, and Embase. Data abstraction was performed while blinded and was followed by meta-(regression)-analyses. We identified 82 studies. Study design related determinants of $\mathrm{SCl}$ pain prevalence reports were: pain definition strictness (mild, moderate, or high), primary study goal (pain study or not), data source (retrospective or not), and, in a limited number of cases, response/attrition rates. While correcting for latter items, populated characteristics found to determine pain prevalence rates were both proportion of patients with a depression and average time after injury (positive correlations). Between-study heterogeneity may remain even after the identification/correction of abovementioned causes of heterogeneity. Pain after $\mathrm{SCl}$ seems to relate to the duration of the injury and depression, yet, major causes of bias in reported pain prevalence are found to be related to the primary study goal (pain study or not), choice of pain definition, and the use of retrospective data.
\end{abstract}




\section{Introduction}

Treatment of Spinal Cord Injury (SCI) related pain is still a major problem (Finnerup 2012a, Haisma 2007, Teasell 2010). The reported prevalences of pain after SCl, however, vary considerably, with rates reported from 19 to as much as 96\% (Dijkers 2009, Finnerup 2001, New 2002, Raissi 2007, Siddall 2003). In a recent, and the only published, systematic review of SCI pain prevalence studies, it was concluded that a definite statement about a pain prevalence could not be made, due to this heterogeneity of the study population (Dijkers 2009). Identifying which factors cause this heterogeneity could reveal important information, as their identification supports their role as relevant pain determinants and/or study confounders. Unfortunately, the authors of the review reported to be unable to identify such factors, yet, this was not the primary aim of their study. By using a more extensive and somewhat different approach, it might have been possible to identify factors causing the heterogeneity in reported SCl pain prevalences.

First, less stringent exclusion criteria could have been adopted. For example, studies were excluded on the basis of reporting on an unsure, or too large (>25\%), portion of non-traumatic SCl. However, pain prevalence has not yet been consistently reported to differ due to $\mathrm{SCl}$ etiology and the influence of such factors can thus better be analyzed for their impact on heterogeneity if studies with more variation in those factors are not excluded (Ibrahim 2012, Mariano 1992, McKinley 2002b). Similarly, studies not explicitly reporting chronic pain problems were excluded. However, pain occurring in $\mathrm{SCl}$ patients is typically of a persistent nature and, regardless, the definition of 'chronic' remains ambiguous (Cruz-Almeida 2005, Purves 1998). And, again, it might have been possible to (partly) resolve the heterogeneity by analyzing the effect of the variation in pain definition strictness/sensitivity on $\mathrm{SCl}$ pain prevalence. Having more studies included in a systematic review might then also result in a more robust analysis of any other factors (Huedo-Medina 2006, Thompson 1994).

Secondly, quality assessment of the prevalence studies can be used by analyzing the impact of individual quality items, instead of an overall total quality scores (Stroup 2000). In this respect, it is especially important to realize that it is still uncertain which study quality items are actually relevant with respect to significantly differing $(\mathrm{SCl})$ pain prevalence rates.

Therefore, in this systematic review, we wanted to further investigate which study-design or studypopulation characteristic(s) might have an impact on the heterogeneity of $\mathrm{SCl}$ pain prevalence by including previously excluded and newly published studies, and performing a detailed metaregression analysis. 


\section{Methods}

A systematic literature review was conducted on studies reporting on $\mathrm{SCl}$ pain prevalence (i.e. descriptive studies and observational studies). As no protocols are available for performing systematic reviews on prevalence studies specifically, we used protocols for systematic reviews of studies on treatment or exposure effects, when applicable (Dickersin 2002, 2008, Stroup 2000). The following databases were used: MEDLINE, Cumulative Index to Nursing and Allied Health Literature (CINAHL), ISI Web of Knowledge, and Embase. The search was wide and the queries comprised of the terms 'pain', 'dysaesthesia', or 'dysesthesia', and 'spinal cord injury/-ies', 'spinal cord disease/-s', or 'tetra-/quadri- or paraplegia'. The abstract and/or publication title were used to identify articles which might fit the topic. Next, the full document was requested, translated to English, and screened whether these met our inclusion criteria or any exclusion criteria. Also, the reference lists from all screened articles were hand searched for any old and/or missed articles. The major clinical research groups on $\mathrm{SCl}$ pain were contacted for any missing publications. Last, the major journals were hand searched (ie. Pain, Spinal Cord/Paraplegia, the Journal of Spinal Cord Medicine, the American Journal of Physical Medicine \& Rehabilitation, and the Journal of rehabilitation research and development/United States Veterans Affairs).

\section{Inclusion criteria}

Studies were included if they reported on the prevalence of pain after $\mathrm{SCl}$, irrespective of the type of prevalence used (i.e. at a single time point or over a certain amount of time). In one case, only an abstract was available and the data from the abstract was used (Rintala 1991).

\section{Exclusion criteria}

If more than $15 \%$ of the included patients had missing data on the occurrence of pain, the study was excluded (e.g. as in a study by Kakulas and co-workers (Kakulas 1990)). In addition, a study was excluded in any of the following conditions:

- only a subtype of $\mathrm{SCl}$ pain was investigated (such as neuropathic pain)

- no demographic data was available on the patients without pain

- the study population was less than fifteen subjects 


\section{Data collection method}

For data abstraction, articles were blinded for journal, authors, involved institutions, dates, countries, and original language. Any references to these items were removed or replaced with dummy items to preserve story integrity. This was done to prevent subjective interpretations and predispositions becoming a source of bias. Blinding was performed in a digital document, if necessary aided by optical character recognition software (ABBYY FineReader 10). This resulted in identically formatted documents, including intact tables and graphics if those provided study results relevant to this review.

\section{Data items}

In addition to the reported pain prevalence, the following population and study characteristics were abstracted, when available (Table II-1). Studies which separately report on pain prevalence rates for population subgroups of patients who all either have or don't have one of the population characteristics we abstracted (e.g. a complete injury) are also split into 'sub-studies', based on these population subgroups (e.g. one sub-study only reflects the pain prevalence of the part of the study population with a complete injury). These sub-studies are used for separate meta-regression analyses of the study population characteristics. These sub-studies are replaced for their main study when their specific characteristic (e.g. prevalence of complete injury) is added to the metaregression analysis. This way, the results are more likely to display the actual relation of this characteristic with the pain prevalence instead of a random relation.

\section{Subgroup formation by pain definitions used}

Because of the potential effect of between-study differences in used pain definitions on the heterogeneity of reported pain prevalence rates, we classified studies in subgroups based on the restrictedness of their used pain definitions. Hence, pain definitions subgroups were made on the basis of the estimated probability that $\mathrm{SCl}$ patients with (mostly minor) pain problems fell below the pain detection threshold. To this end, the pain definitions used per study were abstracted and presented to six pain researchers independently. The researchers were blinded to all other data from the studies (including the reported pain prevalence), and individually allocated pain definitions in three groups of increasing restrictedness. Specifically, they were asked to assign 0,1 , or 2 points for the strictness of the pain definition, indicating:

0: Mild strictness (Hardly excluding any pain cases)

1: Moderate strictness (Excluding some pain cases)

2: High strictness (Excluding many pain cases)

Final scores were based on consensus when unanimity was not met. 


\begin{tabular}{ll}
\hline Population characteristics & Study characteristics \\
\hline Proportion of patients with: & Pain assessment tool \\
Pain interference & Pain definition \\
Pain catastrophizing & Pain recall periods \\
Complete SCl & Primary study goal (Pain study: yes/no) \\
Tetraplegia & Sample size \\
Traumatic SCl & Attrition/Response rate \\
Married/living together & Sample selection method \\
Professional work & Population source (SCl center: yes/no) \\
Depression & Study method \\
Gender ratio & Study method uniformity \\
Age at study & Retrospective data \\
Age at injury & In- and exclusion criteria \\
Time since injury & Publication year \\
Region studied: & Study duration \\
Europe, Canada, and Australia & Description non-responders \\
vs. & Pain classification/taxonomy used; \\
the USA & IASP (Siddall 2000), Bryce/Ragnarsson (Bryce \\
& 2006), or Cardenas (Cardenas 2002) \\
& Reporting of: (each item: yes/no) \\
& Neuropathic or nociceptive pain prevalences \\
& Anatomical pain locations \\
\hline Pablin descriptors \\
\hline
\end{tabular}

Table II-1: Data collected from included studies

\section{Study quality}

From the abstracted data, a quality score for each included study was calculated (maximal score: 18). The quality criteria used were based on those recommended by Leboef-Yde and Lauritsen (Leboeuf-Yde 1995), and Walker (Walker 2000). As the criteria were originally designed for studies on low-back pain, the descriptions were modified to fit the current study on SCI pain (Table II-2). 


\begin{tabular}{|c|c|}
\hline \multirow[t]{3}{*}{$\begin{array}{l}\text { A. Representation of the target } \\
\text { population }\end{array}$} & $\begin{array}{l}\text { 1. The study must at least entail the entire source population, a } \\
\text { random selected sample or a sample which represents the target } \\
\text { population. ( } 2 \text { pts.) }\end{array}$ \\
\hline & $\begin{array}{l}\text { 2. Either the reasons for nonresponses (to study invitation), the } \\
\text { non-responders, a comparison of responders and non- } \\
\text { responders, or the sample and a representative population are } \\
\text { described. ( } 2 \text { pts.) }\end{array}$ \\
\hline & 3. Response rate $\geq 90 \%$ ( 2 pts.) or between $70 \%$ and $90 \%$. (1 pt.) \\
\hline \multirow[t]{3}{*}{ B. Quality of the data } & $\begin{array}{l}\text { 4. Were the data from a pain prevalence study ( } 2 \text { pts.) or from a } \\
\text { study not specifically designed for that purpose. ( } 1 \text { pt.) }\end{array}$ \\
\hline & $\begin{array}{l}\text { 5. Data were collected on an identical fashion for all subjects. ( } 2 \\
\text { pts.) Otherwise (1 pt.) }\end{array}$ \\
\hline & $\begin{array}{l}\text { 6. Data were collected by means of a validated ( } 3 \text { pts.), or non- } \\
\text { validated questionnaire/interview }(2 \text { pts.), or retrospectively by } \\
\text { proxies or medical records. ( } 1 \text { pt.) }\end{array}$ \\
\hline \multirow[t]{4}{*}{ C. Study method/results description } & $\begin{array}{l}\text { 7. A description was given of the target population and the setting } \\
\text { the sample was drawn from. ( } 2 \text { pts.) }\end{array}$ \\
\hline & $\begin{array}{l}\text { 8. Description of the injury (etiology, level, completeness, and } \\
\text { timing), and patient (age, and gender). ( }>3 \text { items }=1 \mathrm{pt} \text {.) }\end{array}$ \\
\hline & 9. Final sample size stated. (1 pt.) \\
\hline & 10. Prevalence recall period should be stated. (1 pt.) \\
\hline
\end{tabular}

Table II-2: Used quality items/criteria (adapted from Leboef-Yde and Lauritsen (Leboeuf-Yde 1995), and Walker (Walker 2000))

Besides analyzing the effects of between-study differences in total quality scores and scores of individual quality items (Table II-2) on heterogeneity, non-stratified continuous data were also analyzed when applicable (e.g. for response rates, population size; see Table 1). Moreover, in a similar way we analyzed the influence of some additional dichotomous and continuous variables possibly reflecting the study quality. These variables were: a difference found in population characteristics between participants and non-participants, study had a cohort design (yes/no), year of publication, and items indicating an extensive pain investigation: 1) the use of the IASP/Siddal, Bryce/Ragnarsson, or Cardenas $\mathrm{SCl}$ pain classification, 2) the report of neuropathic pain prevalence, 3) the report of musculoskeletal pain prevalence, 4) anatomical pain locations, and 5) pain descriptors.

\section{Statistical procedures}

For each study, the standard error of the prevalence rates was calculated, using the standard formula for the standard error of a proportion and the mean of the reported prevalence rates (Chang 2001). Meta-regression analyses were then used to explore sources of bias and heterogeneity. More specifically, possible reasons for (remaining) heterogeneity $\left(I^{2}\right)$ were investigated using a random effects model and assessing linear correlations with reported pain 
prevalence of the overall study quality and the individual study quality items, general study characteristics, and patient/population characteristics. All individual study characteristics and population characteristic were also plotted against the reported pain prevalences in order to identify possible outliers.

For comparisons of means, one way analysis of variance (ANOVA) was used (with Bonferroni post hoc test), or a $t$-test in cases where only two levels of a factor were present. Weighted means were calculated using the size of the study populations and are inversely proportional to the variance of an observation/prevalence (analytic weights). Variations of the mean are given as standard deviations, unless stated otherwise. Statistics were performed using STATA 12 (StataCorp LP, College Station, TX, USA).

\section{Results}

Based on our keywords, the electronic search resulted in 5146 articles which were screened for original $\mathrm{SCl}$ pain prevalence information or references. This resulted in a total of 247 articles. An additional 73 articles were found through searching the references, hand searching and contact with research groups. From the total of 320 articles, 233 articles were excluded, leaving 87 articles for analysis. Reasons for exclusion were: no pain prevalence reported, no original study conducted; only pain patients studied; a too small study population; a subgroup of pain types investigated; overview or case study article.

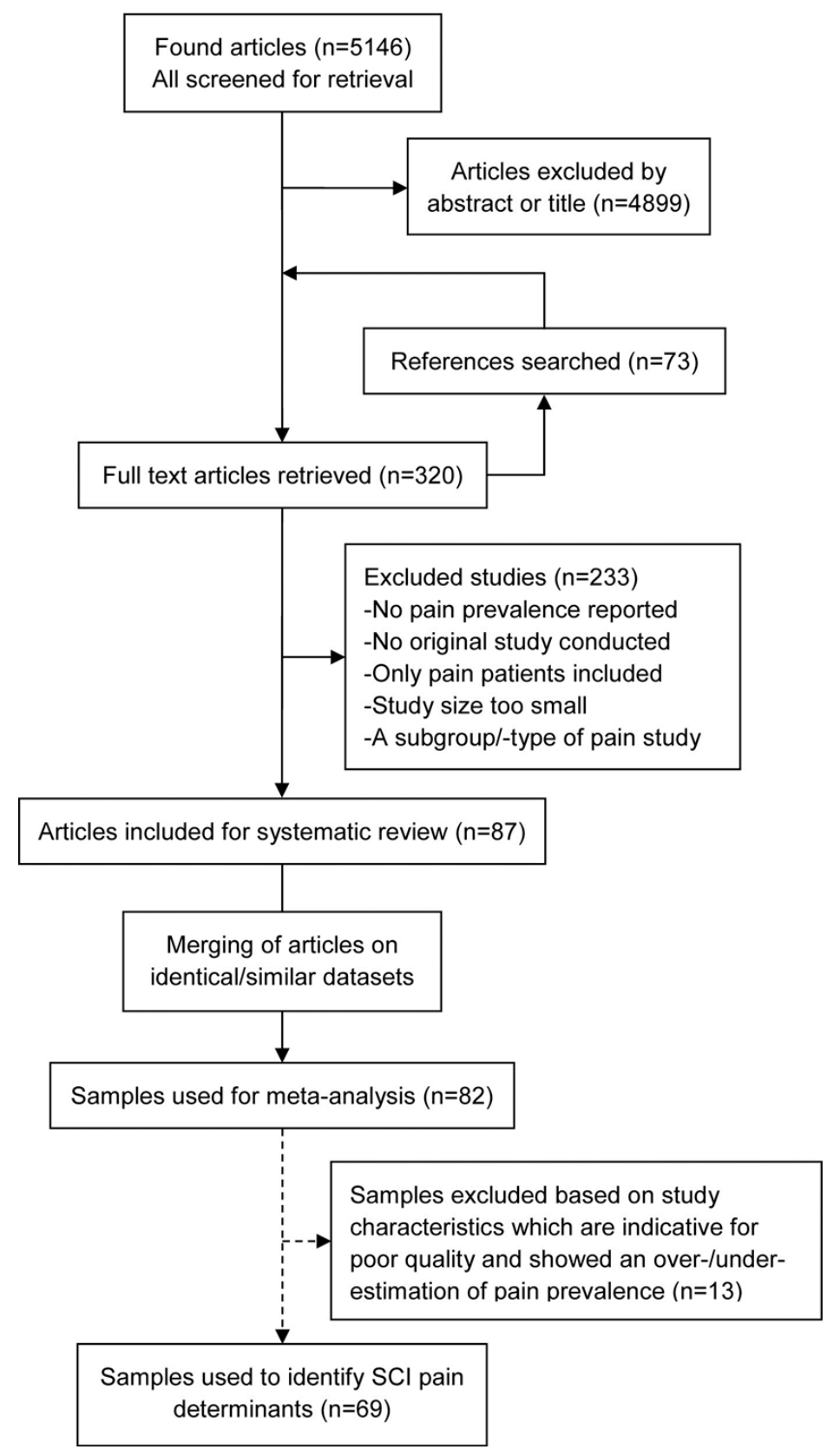

Figure II-1: Flow diagram summarizing systematic search and study inclusion 
In four cases, there were either two or three publications on apparently the same or very similar population/dataset: 1) Walter et al. (Walter 2000, Walter 2002), 2) Jensen et al. and Raichle et al. (Jensen 2005, Jensen 2007, Raichle 2007), 3) Gerhart et al. and Johnson et al. (Gerhart 1992, Johnson 1998), and 4) Klotz et al. and Ville et al. (Klotz 2002, Ville 2001). The study results from the two publications were joined together for this review, which resulted in a total amount of 82 samples (flow diagram: see Fig. II-1). Descriptive overall information per study is given in Table II-5.

\section{Prevalence rates and heterogeneity}

Significant heterogeneity was found between studies $\left(I^{2}=98 \%\right)$. Overall prevalence rate of $\mathrm{SCl}$ pain reported was $61 \pm 20 \%$ (weighted: $57 \pm 18 \%$ ), but due to the large heterogeneity, these numbers should only be used as an indication. In this line, average prevalence rates for the pain definition subgroups, i.e. mild, moderate, and high strictness used for the definition of pain cases were $67 \pm 19 \%$ ( $n=39$, weighted: $69 \pm 15 \%), 59 \pm 18 \%$ ( $n=33$, weighted: $59 \pm 17 \%)$, and $44 \pm 14 \%$ ( $n=11$, weighted: $39 \pm 7 \%$ ), respectively. Prevalence rates differed significantly across the three subgroups (ANOVA $\left.\mathrm{F}_{(2,80)}=6.91, P=0.002\right)$. Pain prevalence rates within subgroups also showed significant heterogeneity (from mild to high pain definition strictness; $l^{2}=97 \%, 97 \%$, and $93 \%$, respectively). Pain definition strictness correlated with reported pain prevalence rates (coef $=-10 \%, P<0.001, l^{2}=96.2 \%, R_{\text {adj }}^{2}=13 \%$; Fig. II-2).

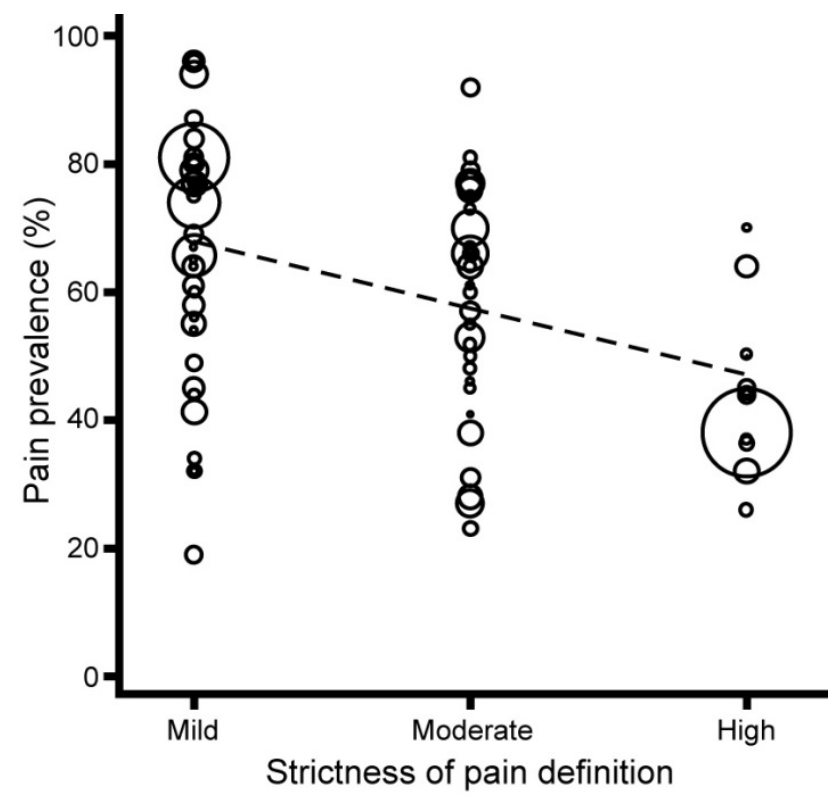

Figure II-2: Correlation of definition strictness with reported pain prevalence. Circle size represents study size. Coefficient: $-10 \%, P=0.001, I^{2}=96.2 \%$

\section{Study quality}

The distribution of the quality scores of the individual studies is shown in Figure II-3 (average quality was $12.1 \pm 2.6$ ). The quality scores of the mild to high pain definition strictness subgroups were $12.5 \pm 0.39$, $12.0 \pm 0.47$, and $12.82 \pm 0.82$, respectively, with no differences across pain definition subgroups $\left(F_{(2,80)}=0.45, \quad P=0.64\right)$. Metaregression analyses performed with 'pain definition strictness' as a covariate, showed a significant correlation between reported pain prevalence and the total quality score of each study (coef $=1.8 \%, \quad P=0.014$, $R_{\text {adj }}^{2}=19 \%$ ). We further investigated which 


\section{Individual study quality criteria}

Two individual quality criteria correlated with reported pain prevalence rates, if corrected for pain definition strictness (i.e. pain definition strictness was added to the meta-regression model as a covariable; results; Table II-3).

First, the quality criterion 'method used to acquire the pain prevalence (validated, nonvalidated questionnaires, or retrospective sources)' was correlated with reported prevalence numbers (coef $=11 \%$, $P=0.002$ ). This correlation is attributed to significantly lower

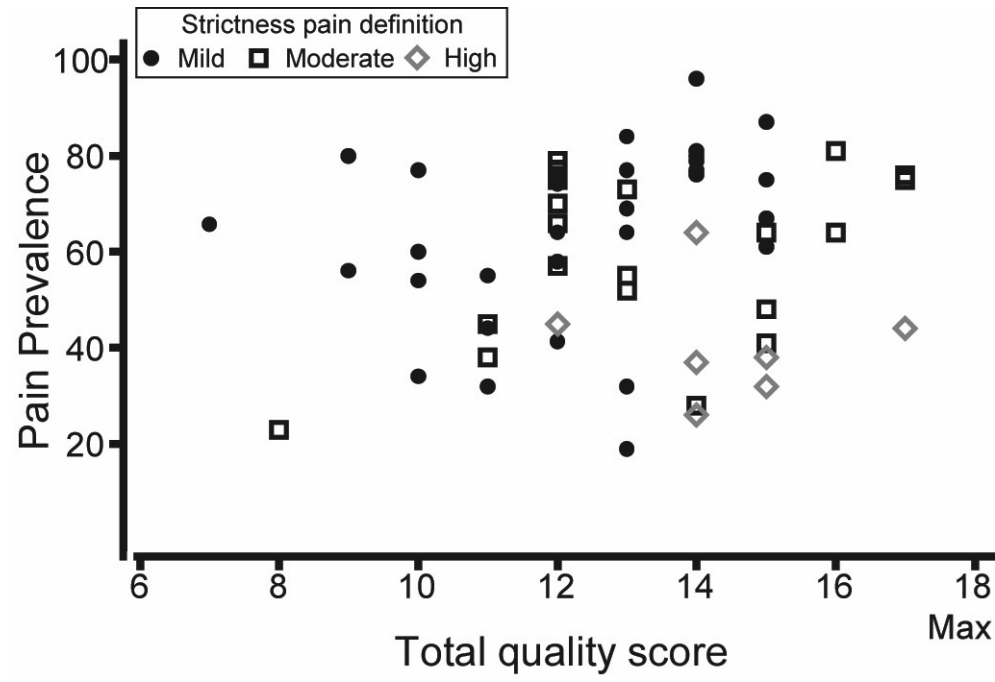

Figure II-3: Distribution of the overall/total study quality scores versus the reported pain prevalences. The different pain definition subgroups are grouped prevalence rates reported by studies using retrospective data (vs. other data sources; coef=-26\%, $\left.P<0.001, I^{2}=95.6 \%, \mathrm{R}_{\mathrm{adj}}^{2}=31 \%\right)$.

Second, the quality criterion 'whether or not the study's primary goal was to report pain prevalence' was positively correlated with reported pain prevalence rates (coef $=18 \%, P<0.001, I^{2}=94.9 \%$, $\left.\mathrm{R}^{2}{ }_{\mathrm{adj}}=36 \%\right)$. Hence, studies with a primary goal to report pain prevalence showed a higher number of patients suffering from pain. Moreover, this correlation was also present in all individual pain definition subgroups. From the pain definition strictness subgroup with hardly any cut-off to the ones with more strict definitions the correlation coefficients were $16 \%(P=0.009), 17 \%(P=0.005)$, and $18 \%(P=0.016)$, respectively (Fig. II-4). Also, the additional items 'whether or not a study reported on neuropathic pain prevalence' and 'whether or not a study reported on neuropathic pain prevalence' were found positively correlated with reported pain prevalence rates. However, the studies reporting on neuropathic and/or musculoskeletal pain prevalences are probably mostly the same studies as those with a primary study goal to report on pain prevalences. Therefore, we added the quality criterion 'whether or not the study's primary goal was to report pain prevalence' as covariable to the regression model and ran the meta-regression analysis again for the variables 'reported neuropathic pain prevalence' and/or 'reported musculoskeletal pain prevalence'. Neither of the latter two variables then remained correlated with pain prevalence, while the quality criterion 
'whether or not the study's primary goal was to report pain prevalence' remained significantly correlated with the reported pain prevalences (coef $=17 \%, P<0.001)$.

\begin{tabular}{|c|c|c|}
\hline \multirow[b]{2}{*}{ Study quality items: } & \multicolumn{2}{|c|}{$\begin{aligned} & \text { Correlation coefficient (\%) w/ Pain Prevalence } \\
& \text { Covariable: } \text { Pain definition strictness } \\
& \text { (Mild, Moderate, or High) } \\
& \text { Coefficient } \leq-8.7 \% ; P \leq 0.003\end{aligned}$} \\
\hline & Coefficient $(\%)$ & $\begin{array}{c}\mathrm{l}^{2} \\
\text { (\% residual variation } \\
\text { due to } \\
\text { heterogeneity) }\end{array}$ \\
\hline None & & 96.2 \\
\hline 1. Representative study population & 2.8 & 96.1 \\
\hline 2. Non-responders described & -0.1 & 96.2 \\
\hline $\begin{array}{l}\text { 3. Attrition / Response rate (continuous values) } \\
<90 \% \text { vs } \geq 90 \%\end{array}$ & $\begin{array}{c}-2.6\left(-0.1^{\dagger}\right) \\
-3.6\end{array}$ & $\begin{array}{c}96.2\left(96.0^{\dagger}\right) \\
96.2\end{array}$ \\
\hline 4. Primary study goal is pain prevalence & $17.6^{* * *}$ & 94.9 \\
\hline 5. Uniform data collection & 4.1 & 96.2 \\
\hline $\begin{array}{l}\text { 6. Data acquisition } \\
\text { Other vs retrospective } \\
\text { Other vs non-validated questionnaire } \\
\text { Other vs validated questionnaire }\end{array}$ & $\begin{array}{l}11.1^{\star \star} \\
-26.0^{\star \star *} \\
7.1 \\
5.4\end{array}$ & $\begin{array}{l}96.0 \\
95.6 \\
96.2 \\
96.2\end{array}$ \\
\hline 7. Description of population and its source & 7.9 & 96.2 \\
\hline 8. More detailed description of injury & 8.0 & 96.2 \\
\hline 9. Final sample size stated & $N / A^{\ddagger}$ & \\
\hline 10. Pain recall period stated & 4.5 & 96.2 \\
\hline \multicolumn{3}{|l|}{ Additional study characteristics: } \\
\hline Survey study (no vs. yes) & 7.3 & 96.2 \\
\hline Year of publication & $0.3^{*}$ & 96.1 \\
\hline $\begin{array}{l}\mathrm{SCl} \text { pain classification system used (no vs. } \\
\text { yes) }\end{array}$ & 11.9 & 96.2 \\
\hline Cohort study ( no vs. yes ) & 9.2 & 96.1 \\
\hline Patients from SCl center (no vs. yes) & -3.4 & 96.2 \\
\hline $\begin{array}{l}\text { Detailed pain info given (no vs. yes) } \\
\text { Neuropathic pain prevalence } \\
\text { Musculosckeletal pain prevalence } \\
\text { Anatomical locations } \\
\text { Pain descriptors }\end{array}$ & $\begin{array}{l}11.5^{(* *) \int} \\
12.6^{(*) \int} \\
8.2 \\
9.8\end{array}$ & $\begin{array}{l}95.8 \\
96.0 \\
96.1 \\
96.2\end{array}$ \\
\hline
\end{tabular}

Table II-3: Meta-regression results of pain prevalence rates and individual study quality items

+ Studies not reporting attrition rates excluded ( $n=61$ remaining),

‡ All studies stated final sample size,

S Correlation lost significance when "quality item 4: Primary study goal is pain prevalence" was added as a covariable to the model. ${ }^{*} \mathrm{P}<0.05, * * \mathrm{P}<0.01$. 
The additional study characteristic 'year of publication' also correlated with the reported pain prevalence rates (coef $=0.3 \%, \quad P=0.025$ ). However, after exclusion of the five studies published before 1960 (weighted prevalence: $32.6 \pm$ SD21\%; study quality: $8.0 \pm$ SD1.4), the relation between 'year of publication' and reported pain prevalence was lost (coef $=0.0 \%$, $P=0.928)$.

Hence, studies with retrospectively acquired data and studies from

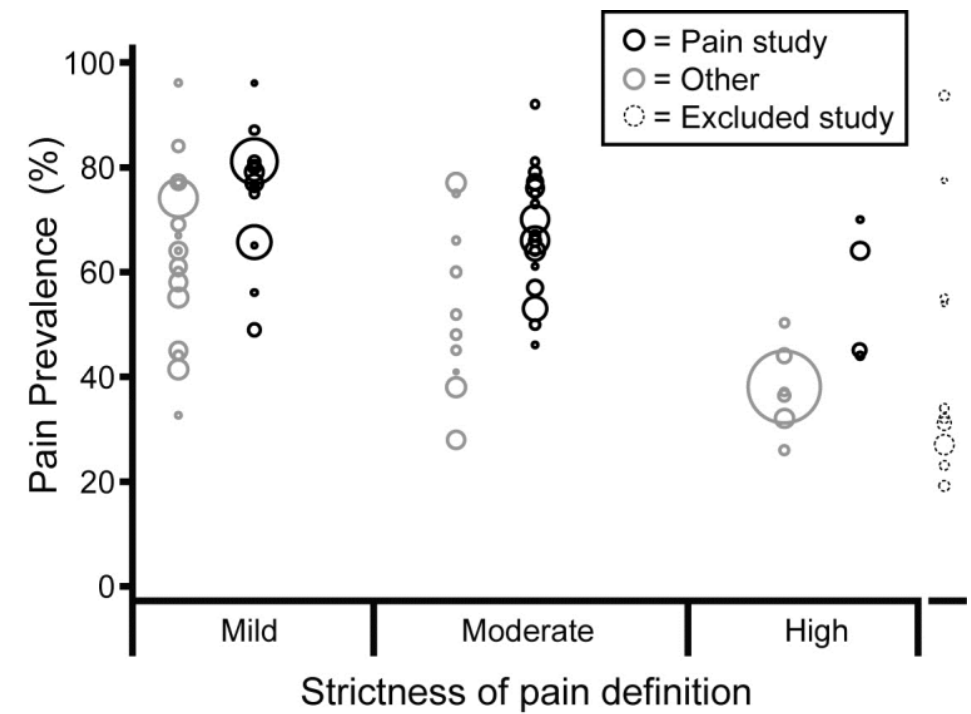

Figure II-4: The relation between de primary study goal (pain study or not) and the pain prevalence reported. Retrospective and old studies $(<1960)$ excluded and shown as interrupted circles before 1960 were excluded from further analyses ( $n=10$ excluded). In addition, when observing the graph in which the reported prevalence rates are plotted against the response/attrition rate (Fig. II5), three studies raised doubts on whether or not they should be interpreted as outliers (marked

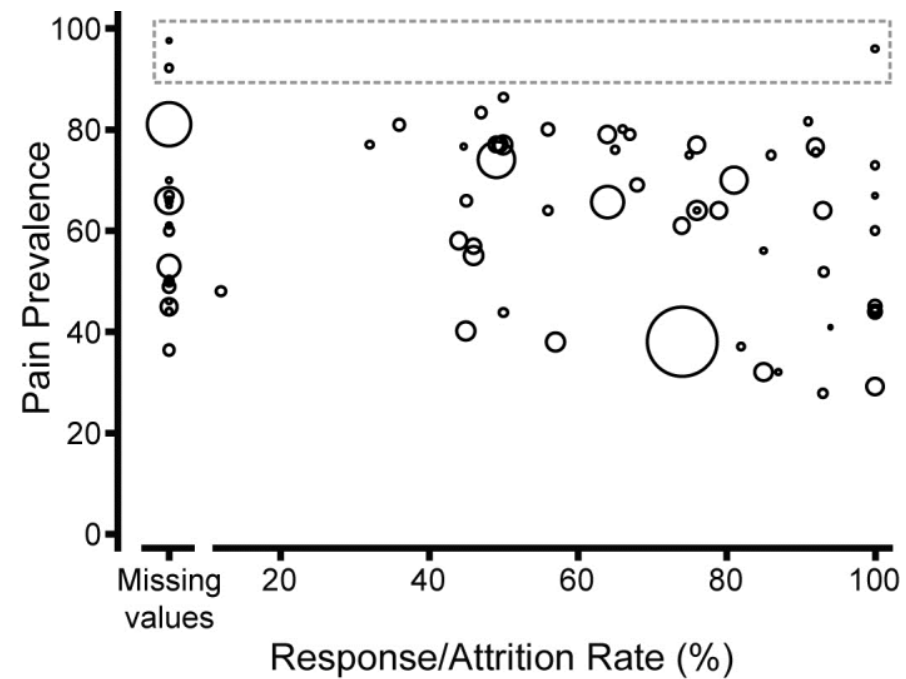

Figure II-5: The relation between de response rate and the pain prevalence reported. Note that the three studies with the highest prevalence rates (marked with the dotted box) are also studies which either reported a $100 \%$ response rate (mostly due to unlocatable potential participants), no response rates, and contain only small study populations. Retrospective and studies from before 1960 excluded with interrupted/dotted rectangle). These studies were small, were reporting either no response rates or (in one case) a $100 \%$ response rate, and reported the highest prevalence rates of all remaining studies. Therefore, we decided to exclude them for further analyses $(n=3$ excluded, $n=69$ remaining). In the excluded study with a $100 \%$ response rate all 54 eligible patients who the investigators were able to get in contact with had been included, other potential study participants were not locatable (Raissi 2007). 
Then, after exclusion of old (i.e. published before 1960) and retrospective studies, and correcting for the covariates 'primary study goal (pain study or not)' and 'pain definition strictness' $\left(l^{2}=93.1 \%\right.$, $\mathrm{R}_{\mathrm{adj}}^{2}=45 \%$ ), both the item 'whether or not there was a representative study population' as well as 'whether or not a study was a survey study' correlated with the reported pain prevalences (coef $=5.2 \%, P=0.003 \&$ coef $=7.6 \%, P=0.011$, respectively). This remained true when both items were simultaneously loaded in the meta-regression model (coef $=4.5 \%, P=0.008$ \& coef $=6.1 \%, P=0.035$, respectively). However, both or either of the latter items lost their correlation with the pain prevalence rates when adding any of the population characteristics (Table II-1) to the regression model, indicating a weak correlation, which made us decide to exclude them from the metaregression model.

Next, when loading the total quality scores of individual studies to the last-mentioned metaregression model showed that total quality scores no longer correlated with pain prevalence rates (coef $=0.9 \%, P=0.18$ ). Moreover, this was also true when the original total quality score was recalculated by subtracting the quality points assigned for the quality item 'primary study goal' (coef $=0.7 \%, P=0.21$ ).

\section{Possible determinants for $\mathrm{SCl}$ pain}

With the adjustments described above (i.e. inclusion of the four covariates 'pain definition strictness' and 'primary study goal' in the meta-regression model, and exclusion of retrospective studies, studies from before 1960, and the three outliers on the response-rate plot) meta-regression analyses were performed in an attempt to uncover possible determinants of the reported pain prevalence by loading individual study population characteristics in to the model. The population characteristics showing a significant correlation with the reported pain prevalence were 'Time after injury' (coef $=0.9 \%, \mathrm{P}=0.03, \mathrm{l}^{2}=95.1 \%, \mathrm{R}_{\mathrm{add}}^{2} \mathrm{j}=49 \%, \mathrm{n}=34$ ) and 'Depression prevalence' (coef $=0.6 \%$, $\mathrm{P}=0.003, I^{2}=69.7 \%, \mathrm{R}_{\text {adj }}^{2}=81 \%, \mathrm{n}=13$; Table II-4).

Then, remaining study quality items, study characteristics, or population characteristics are individually loaded in any of the two meta-regression models of the significantly correlated population characteristics, no additional correlations were identified. This was also true for the study characteristics 'representative study population' and 'whether or not a study was a survey study', which were earlier found to correlate with pain prevalence rates (see section above). We attempted to combine 'Depression prevalence' and 'Time after injury' in the meta-regression model, but due to insufficient studies reporting on both population characteristics, this was not possible. 


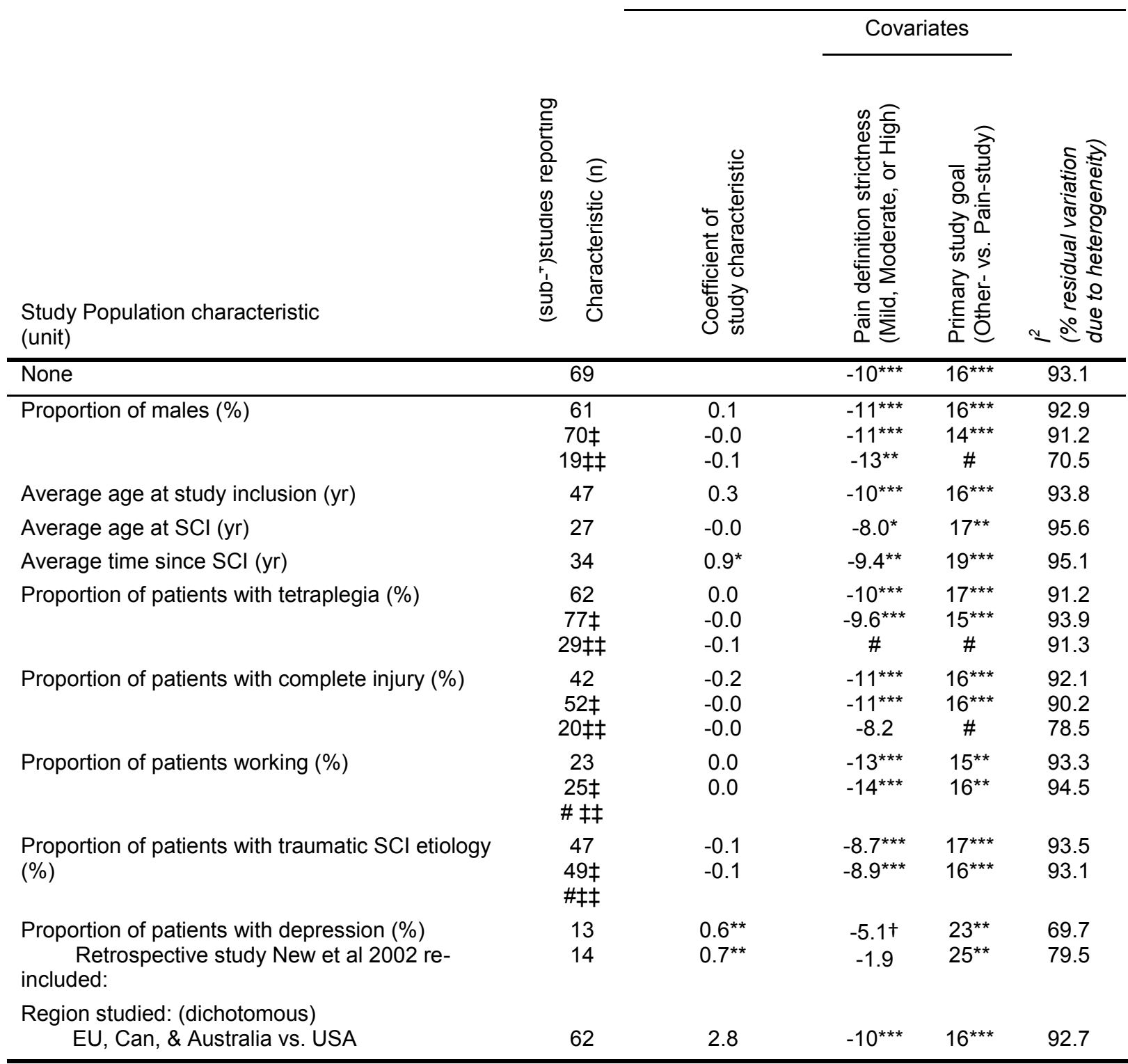

Table II-4: Meta-regression analyses of pain prevalence rates and individual study population characteristics

Retrospective studies, studies from before 1960, and response/attrition rate outliers are excluded.

₹ Studies which separately report on pain prevalence rates for population subgroups of patients who all either have or don't have the analyzed characteristic (e.g. a complete injury) are split into 'sub-studies', based on these population subgroups (e.g. one sub-study only reflects the pain prevalence of the part of the study population with a complete injury)

¥¥ Sub-studies only

* $\mathrm{P}<0.05, * * \mathrm{P}<0.01, * * * \mathrm{P}<0.001,+\mathrm{P}<0.10$

\# Removed from model; most substudies fall into only one of the categories of the covariable. 
After removal of 'pain definition strictness' and 'primary study goal' from the meta-regression model, 'Depression prevalence' remained significantly correlated with the reported pain prevalences (coef $=0.7 \%, P=0.03, l^{2}=86.5 \%, R_{a d j}^{2}=37 \%$ ), which was not true for 'Time after injury' (coef $=0.7 \%$, $\mathrm{P}=0.15, l^{2}=96.6 \%, \mathrm{R}_{\text {adj }}^{2} \mathrm{j}=3 \%$ ). Last, after including the three previously excluded studies (based on being outliers on the response-rate plot; Fig. II-5) 'Time after injury' no longer correlated with the reported pain prevalence rates (coef $=0.6 \%, P=0.08$ ). Otherwise, no major differences to the values reported in Table II-4 were noted.

\section{Discussion}

This is the first meta-analysis which systematically explored causes of the large heterogeneity in reported SCl pain prevalences. Foremost, we found that (1) pain definition strictness (stratified as mild, moderate, or high) showed a strong negative correlation with reported $\mathrm{SCl}$ pain prevalences (i.e. more strict definitions were associated with lower prevalence), and (2) 'whether or not a study was primarily a pain study' had a strong positive correlation with pain prevalence rates.

Furthermore, a positive correlation between pain prevalence and depression rates was found although this should be interpreted with some care, as not all of the included studies reported on depression prevalence rates $(n=13)$. Only rarely official criteria for depression are used, and most studies use an arbitrary cut-off score for the amount and/or severity of depressive symptoms. Much has been written about the well-established relationship between (chronic) pain and depression (Bair 2003, Henningsen 2006, Jann 2007). A bi-directional relationship, where depression increases the risk for higher pain levels and vice-versa, seems to be present for patients suffering from a disability (Gayman 2011, Saunders 2012), although others dispute the notion that there is any relation between depression and pain in SCl patients (Hassanpour 2012). Yet, the positive correlation found between $\mathrm{SCl}$ pain prevalence and depression prevalence is in line with a recently published five year longitudinal study performed in SCI patients, were depression onset was related to an increase in pain (Hoffman 2011).

Time after injury also came forward as a putative $\mathrm{SCl}$ pain determinant in this meta-analysis, with higher pain prevalences reported in studies performed longer after the onset of the SCl. Not enough studies reported on both time after injury and depression to see if there was a correlation between these variables, to exclude co-linearity. However, average depression prevalence over the first five years after $\mathrm{SCl}$ was reported to change very little, suggesting little overlap between depression and time after injury in the increase in pain (Hoffman 2011). In a prospective study, pain prevalence was indeed shown to increase in time, when compared between six months and five years (Siddall 2003). This effect is mostly due to the onset of pain shortly after the six months measurement as 
differences are less evident when comparing prevalence rates at two years post injury with those at six years (Jensen 2005). It is thus interesting that it was shown that, although depression and pain were independent in the acute phase after $\mathrm{SCl}$ (rehabilitation admission; week 2-3 post injury), a stronger relationship developed over time (Cairns 1996).

Differences in pain definitions are a clear source of heterogeneity between pain prevalence reports. It is thus unfortunate that it is impossible to predict more precisely how each difference in definition (recall period, duration, choice of words etc.) influences the reported prevalence. For meta-analyses of prevalence studies the question remains to what extent the exclusion of studies with a somewhat differing definition weighs up to the subsequent loss of statistical power (smaller size) of metaanalyses. Nonetheless, one needs to keep in mind that classification of pain definitions does not circumvent problems arising from the lack of reporting on the complete pain definitions with all its attributes as used in a study (e.g. recall period, frequency, duration, intensity, etc). Moreover, it is not always sure if reported attributes were consistently applied throughout the study.

Quality of studies to be included in systematic reviews is a major concern, but quality is not easily defined (Schulz 1995). It is doubtful that elimination of studies with below-average 'overall quality scores' prevents formulation of wrong conclusions (Moher 1998). Quality scores can be misleading, especially when designed in an ad-hoc manner (Emerson 1990, Greenland 1994). This is especially true for meta-analyses on pain prevalence studies, because no research is done on the influence of individual quality items. Strikingly, we found that higher quality studies failed to show increasingly consistent prevalence rates. In any case, it seems more important to analyze the impact of individual quality items instead of the overall quality score (Friedenreich 1994, Hasselblad 1992, Juni 1999).

In our study, only two quality items out of ten (i.e. primary study goal and method of data acquisition; retrospective or not) showed to be of main importance on the variance between reported $\mathrm{SCl}$ pain prevalence. First, whether or not the primary study goal was to study pain was positively correlated with reported $\mathrm{SCl}$ pain prevalence rates. This could mean that $\mathrm{SCl}$ pain prevalence studies tend to overestimate pain prevalence rates, for example, because patients without pain are less interested to participate in pain studies (Locker 1988, Rupp 2002, Schmidt 2011). Another explanation could be that the difference is a sign of publication bias (Dwan 2008). Second, retrospective studies tended to underreport pain prevalence rates. A not very surprising finding, since pain cases in retrospective studies are not actively sought and remain undetected.

The reported prevalences of complete injuries showed no trend for a relation with pain prevalence rates. Both clinical and preclinical data support the concept that specific damage and preservation of 
spinal cord structures are of particular relevance to (especially below-level) $\mathrm{SCl}$ pain (Finnerup 2003a, Finnerup 2007, Hall 2010), but these reports are yet inconclusive due to inconsistencies (Siddall 1999, Siddall 2003, Werhagen 2004, Werhagen 2007, Zeilig 2012). Some, but limited, damage to the spinothalamic tract is suggested to be important for the onset of pain, as below-level hypersensitivity seems to be a predictor for spontaneous central neuropathic pain (Finnerup 2012b, Zeilig 2012). Surprisingly, even in patients clinically having a complete injury some remaining fibers in the spinothalamic tract might play a role in below-level pain (Garcia-Larrea 2002, Zeilig 2012). Also level of injury (i.e. percentage tetraplegia) also did not appear as a determinant for $\mathrm{SCl}$ pain. The largest prospective cohort study performed on $\mathrm{SCl}$ pain merely reported a slight difference in pain prevalence between tetra- and paraplegic patients (Siddall 2003).

With respect to our use of general/overall pain, we recognize that a sole $\mathrm{SCl}$ pain entity does not exist, and that determinants we identified to be (or not to be) relevant for the prevalence of 'any pain', could thus be biased due to differences in frequencies of different pain syndromes underlying our overall pain prevalences. Yet, different pain syndromes might arise from a multitude of pathophysiological processes which are not all fully understood (Siddall 2002, Widerstrom-Noga 2009). Different processes might overlap because of final common pathways, such as neuronal hyperexcitability at different levels of the central nervous system or psycho-cognitive traits (CruzAlmeida 2009, Finnerup 2003b, Saab 2009, Zhao 2007). Therefore, determinants for any SCI pain should also be looked for in the larger general SCl pain population. Admittedly, the success of this approach may largely rely on (very) large numbers of patients, or, as done here, on meta-analyses. Of course, current clinical SCI pain classifications could also be useful to more selectively search for pain determinants, but pathophysiological overlap between these pain types might remain (Bryce 2006, Putzke 2003b, Ragnarsson 1997, Siddall 1997).

Last, we found residual heterogeneity, but one relevant method often used in meta-analyses might not be fully appropriate, at least when applied on pain prevalence studies. The standard approach to estimate within-study variation assumes that the size of the study is the most important factor for the accuracy of the prevalence estimate of an included study, which strongly determines the impact (and possible heterogeneity) of this study in the meta-analysis. This is, however, arguable in case of pain prevalence studies, as many equivocal factors come into play when one would like to accurately assess pain prevalence (attributes of pain definition, assessment method, etc). It does seem inappropriate that the within-study variation of pain prevalence rates are estimated with the same stringency and manner as for less ambiguous outcomes as, for example, mortality rates. Also, when using the standard formula for calculating the within-study variance estimate of a reported 
prevalence the variance automatically gets much narrower when the prevalence approaches its extremes (i.e. $0 \%$ or $100 \%$ ). However, a priori, in any random SCl patient population it is very unlikely that all patients experience pain. Hence, should we not estimate the variation of a pain prevalence differently to get a more realistic analysis of residual heterogeneity?

\section{Conclusion}

Variance in $\mathrm{SCl}$ pain prevalences is mostly due to the strictness of pain the definition used, whether or not the study was a pain study, but is also due to the use of retrospective data, the time since injury, and the prevalence of depression. Future $\mathrm{SCl}$ pain studies should clearly and more uniformly define and report pain definitions, report prevalences of depressive symptoms and time since injury and investigate them as pain determinants in $\mathrm{SCl}$ patients, and take special care not include bias by selecting for pain patients in pain (prevalence) studies. The identification of SCl pain determinants will be indispensable for the development of therapeutic approaches that effectively control SCI pain and ameliorate the quality of life of many SCI patients.

Table II-5: Included studies and their main characteristics

\begin{tabular}{|c|c|c|c|c|c|c|c|c|c|c|c|}
\hline $\begin{array}{l}\text { Article } \\
\text { (by year) }\end{array}$ & 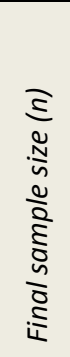 & 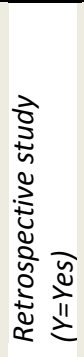 & 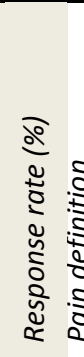 & 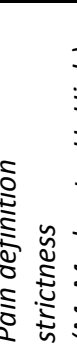 & 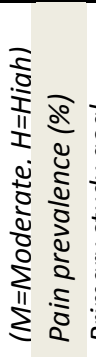 & 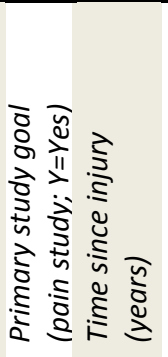 & 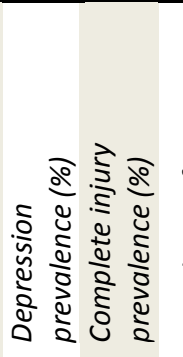 & 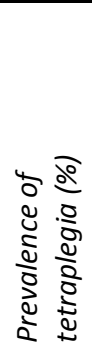 & 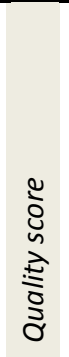 & 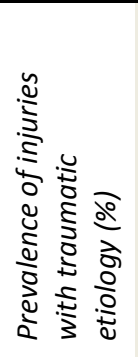 & $\begin{array}{r}\text { Pain definition } \\
\text { (patient setting) }\end{array}$ \\
\hline $\begin{array}{l}\text { Davis } \\
1947\end{array}$ & 471 & & & $\bar{M}$ & 27 & $\bar{Y}$ & & 16 & 8 & & $\begin{array}{l}\text { Pain severely enough to } \\
\text { consider active steps for its } \\
\text { relief }\end{array}$ \\
\hline $\begin{array}{l}\text { Kuhn } \\
\qquad 1947\end{array}$ & 113 & Y & 86 & M & 23 & & 71 & 9 & 8 & 100 & $\begin{array}{l}\text { Painful sensations; tingling } \\
\text { and burning to severe } \\
\text { agonizing, knife-like pains } \\
\text { (rehabilitation admission) }\end{array}$ \\
\hline $\begin{array}{l}\text { Munro } \\
\quad 1948\end{array}$ & 99 & $Y$ & 47 & & 34 & & & & 10 & & Pain (admission; at discharge) \\
\hline $\begin{array}{l}\text { Munro } \\
\quad 1950\end{array}$ & 228 & Y & & M & 31 & & 57 & 7 & 6 & & Pain (admission) \\
\hline $\begin{array}{r}\text { Botterell } \\
1954\end{array}$ & 125 & & & & 94 & Y & 40 & 16 & 8 & 100 & Pain or discomfort \\
\hline $\begin{array}{l}\text { Kaplan } \\
\qquad 1962\end{array}$ & 44 & & 85 & & 56 & Y & & & 9 & 46 & Pain (outpatient) \\
\hline $\begin{array}{l}\text { Burke } \\
\qquad 1973\end{array}$ & 214 & & 100 & $\mathrm{H}$ & 45 & Y & & & 12 & & $\begin{array}{l}\text { Continuous pain complaints } \\
\text { and/or regularly analgesic use } \\
\text { (admission) }\end{array}$ \\
\hline
\end{tabular}




\begin{tabular}{|c|c|c|c|c|c|c|c|c|c|c|c|c|c|}
\hline $\begin{array}{l}\text { Article } \\
\text { (by year) }\end{array}$ & 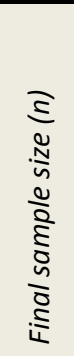 & 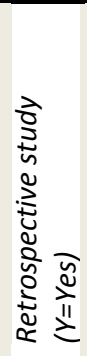 & 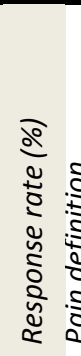 & $=$ & 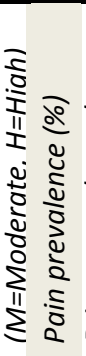 & 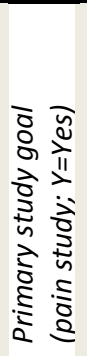 & 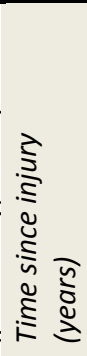 & 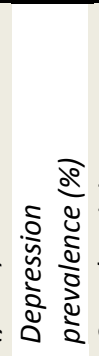 & 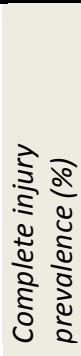 & 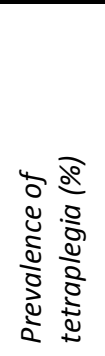 & 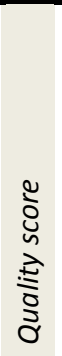 & 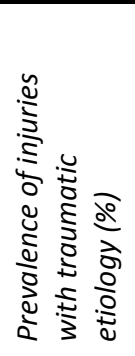 & $\begin{array}{r}\text { Pain definition } \\
\text { (patient setting) }\end{array}$ \\
\hline $\begin{array}{c}\text { Nepomuceno } \\
1979\end{array}$ & 200 & & 56 & & 80 & $\mathrm{Y}$ & & & & 45 & 9 & 100 & $\begin{array}{l}\text { Painful unpleasant sensations } \\
\text { (outpatients) }\end{array}$ \\
\hline $\begin{array}{l}\text { Richards } \\
1980\end{array}$ & 75 & & 32 & & 77 & & & & 71 & 37 & 10 & 88 & $\begin{array}{l}\text { Pain subsequent to } \mathrm{SCl} \\
\text { (outpatients) }\end{array}$ \\
\hline $\begin{array}{l}\text { Lamid } \\
\qquad 1985\end{array}$ & 64 & & & $\mathrm{H}$ & 44 & $Y$ & & & & & 9 & & $\begin{array}{l}\text { Significant pain and reduced } \\
\text { activity (mostly admission) }\end{array}$ \\
\hline $\begin{array}{l}\text { Woolsey } \\
1986\end{array}$ & 100 & & & M & 67 & Y & & & & 48 & 10 & & $\begin{array}{c}\text { Pain of which the patient is at } \\
\text { least aware }\end{array}$ \\
\hline $\begin{array}{l}\text { Rose } \\
1988\end{array}$ & 885 & & 81 & $M$ & 70 & Y & & & 42 & 35 & 12 & & $\begin{array}{l}\text { Pain; either at or below the } \\
\text { neurological level of injury } \\
\text { (mostly outpatient) }\end{array}$ \\
\hline $\begin{array}{l}\text { Beric } \\
\qquad 1990\end{array}$ & 178 & & & & 49 & Y & & & & 70 & 8 & 100 & $\begin{array}{l}\text { Unpleasant sensations for at } \\
\text { least three weeks, after injury }\end{array}$ \\
\hline $\begin{array}{l}\text { Frisbie } \\
1990\end{array}$ & 66 & & 100 & M & 73 & Y & 16 & & & & 13 & 100 & Altered sensations and pain \\
\hline $\begin{array}{l}\text { Krause } \\
1990\end{array}$ & 347 & & 79 & & 64 & & 15 & & & 57 & 12 & 100 & $\begin{array}{l}\text { Not no pain (mostly } \\
\text { outpatient) }\end{array}$ \\
\hline $\begin{array}{r}\text { Lundqvist } \\
1991\end{array}$ & 98 & & 93 & $\mathrm{H}$ & 26 & & 2.3 & & 37 & 48 & 14 & 100 & $\begin{array}{l}\text { Pain associated with } \mathrm{SCl} \\
\text { (outpatient) }\end{array}$ \\
\hline $\begin{array}{l}\text { Rintala } \\
1991\end{array}$ & 640 & & & M & 53 & Y & & & & & 8 & 100 & $\begin{array}{l}\text { Pain severely enough to } \\
\text { impair activities of daily living } \\
\text { (admission) }\end{array}$ \\
\hline $\begin{array}{r}\text { Summers } \\
1991\end{array}$ & 54 & & 75 & $M$ & 75 & & 12 & & & 35 & 12 & & Frequent pains (outpatient) \\
\hline $\begin{array}{l}\text { Brooks } \\
1992\end{array}$ & 84 & & 100 & & 60 & & & 30 & 62 & 19 & 10 & & Pain problem (outpatient) \\
\hline $\begin{array}{r}\text { Fenollosa } \\
1993\end{array}$ & 145 & & 45 & $M$ & 66 & Y & & & 57 & 26 & 12 & 83 & Pain (outpatient) \\
\hline $\begin{array}{c}\text { Knutsdottir } \\
1993\end{array}$ & 45 & & 76 & & 64 & & & & 29 & 56 & 13 & 100 & $\begin{array}{l}\text { Chronic pain; a burning, } \\
\text { sharp, aching, throbbing, or } \\
\text { shooting, etc, sensation for }> \\
6 \text { months (mostly outpatient) }\end{array}$ \\
\hline $\begin{array}{l}\text { Craig } \\
\qquad 1994\end{array}$ & 41 & & 87 & & 32 & & 0.5 & & 74 & 51 & 13 & 100 & $\begin{array}{l}\text { Pain of }>=\text { moderate severity, } \\
\text { after acute phase of } \mathrm{SCl} \\
\text { (mostly outpatient) }\end{array}$ \\
\hline $\begin{array}{r}\text { Stensman } \\
1994\end{array}$ & 17 & & 94 & $M$ & 41 & & & 35 & 100 & 59 & 15 & 100 & Not no pain (first admission) \\
\hline $\begin{array}{l}\text { Anke } \\
1995\end{array}$ & 46 & & & M & 46 & Y & 0.6 & & 39 & 50 & 13 & 100 & $\begin{array}{c}\text { >= Moderate pain in previous } \\
\text { week (mainly at last week } \\
\text { rehabilitation admission) }\end{array}$ \\
\hline
\end{tabular}




\begin{tabular}{|c|c|c|c|c|c|c|c|c|c|c|c|c|c|}
\hline $\begin{array}{l}\text { Article } \\
\text { (by year) }\end{array}$ & 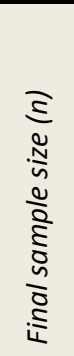 & 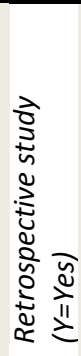 & 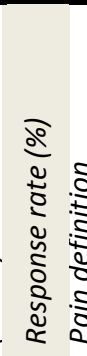 & 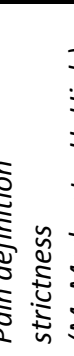 & 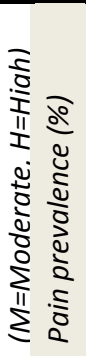 & 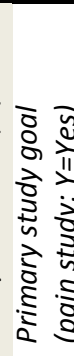 & 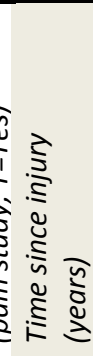 & 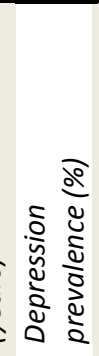 & 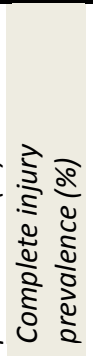 & 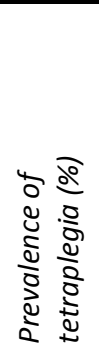 & 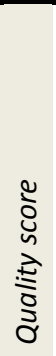 & 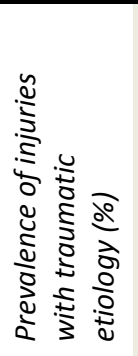 & $\begin{array}{r}\text { Pain definition } \\
\text { (patient setting) }\end{array}$ \\
\hline $\begin{array}{l}\text { Levi } \\
1995\end{array}$ & 353 & & 93 & $\mathrm{H}$ & 64 & $Y$ & 11 & & 40 & 43 & 14 & 100 & $\begin{array}{l}\text { Long-standing/chronic pain, } \\
\text { being a significant } \\
\text { problem/having a continuous } \\
\text { negative impact on quality of } \\
\text { life (mostly outpatient) }\end{array}$ \\
\hline $\begin{array}{l}\text { Anson } \\
1996\end{array}$ & 348 & & & & 45 & & & & & 56 & 8 & 91 & $\begin{array}{l}\text { Not no pain (first } \\
\text { rehabilitation admission) }\end{array}$ \\
\hline $\begin{array}{l}\text { Cairns } \\
1996\end{array}$ & 68 & & 65 & & 76 & $Y$ & 0.1 & 46 & 56 & 34 & 14 & & Pain (outpatient) \\
\hline $\begin{array}{r}\text { Kennedy } \\
1997\end{array}$ & 76 & & 66 & & 80 & $Y$ & 0.1 & & & 32 & 14 & 100 & $\begin{array}{l}\text { Pain during admission (first } \\
\text { rehabilitation admission) }\end{array}$ \\
\hline $\begin{array}{l}\text { New } \\
1997\end{array}$ & 24 & & & & 96 & $Y$ & & & 37 & 54 & 9 & 79 & $\begin{array}{l}\text { Not no pain (6 weeks post } \\
\text { injury, admission) }\end{array}$ \\
\hline $\begin{array}{r}\text { Stormer } \\
1997\end{array}$ & 901 & & & $M$ & 66 & $Y$ & & & & 58 & 9 & & $\begin{array}{l}\text { Pain or distressing } \\
\text { dysaesthesia, for }>=3 \text { months } \\
\text { or }>=6 \text { months with } \\
\text { remissions and relapses }\end{array}$ \\
\hline $\begin{array}{l}\text { Demirel } \\
1998\end{array}$ & 47 & & & M & 61 & $Y$ & & & 32 & 23 & 10 & 96 & $\begin{array}{l}\text { Pain related to the injury } \\
\text { during past } 4 \text { weeks } \\
\text { (outpatient) }\end{array}$ \\
\hline $\begin{array}{l}\text { Johnson } \\
1998 \\
\text { \& Gerhart } \\
1992\end{array}$ & 357 & & 100 & M & 28 & & 1 & 2 & & 53 & 14 & 100 & $\begin{array}{l}\text { Pain lasting or recurring for } \\
>=6 \text { months, not altered } \\
\text { sensations (outpatient) }\end{array}$ \\
\hline $\begin{array}{l}\text { Post } \\
1998\end{array}$ & 315 & & 74 & & 61 & & 3.6 & & & 41 & 15 & 73 & $\begin{array}{l}\text { Perceived health problem: } \\
\text { pain (outpatient) }\end{array}$ \\
\hline $\begin{array}{r}\text { Rintala } \\
1998\end{array}$ & 77 & & 92 & $M$ & 75 & $Y$ & 13.3 & & & 51 & 17 & 100 & $\begin{array}{c}\text { >= Moderate pain in previous } \\
\text { week (during rehabilitation } \\
\text { admission) }\end{array}$ \\
\hline $\begin{array}{r}\text { McKinley } \\
1999\end{array}$ & 41 & $Y$ & 84 & & 54 & & 0 & & 56 & 20 & 10 & 100 & Long-lasting pain (outpatient) \\
\hline $\begin{array}{l}\text { Meyers } \\
1999\end{array}$ & 114 & & 12 & M & 48 & & 12.1 & 42 & & 83 & 15 & & $\begin{array}{l}\text { Any pain (of either } \\
\text { musculoskeletal, visceral, or } \\
\text { neuropathic origin), as } \\
\text { established through } \\
\text { interview, at } 6 \text { months after } \\
\text { injury (outpatient) }\end{array}$ \\
\hline $\begin{array}{l}\text { Siddall } \\
1999\end{array}$ & 100 & & 56 & M & 64 & $\mathrm{Y}$ & 0.2 & & 36 & 51 & 16 & 100 & $\begin{array}{l}\text { Pain problem (mostly } \\
\text { outpatient) }\end{array}$ \\
\hline $\begin{array}{l}\text { Turner } \\
1999\end{array}$ & 164 & & 36 & & 81 & $\mathrm{Y}$ & 12.1 & & 49 & 47 & 14 & 72 & $\begin{array}{l}\text { Chronic pain problem during } \\
\text { previous year (outpatient) }\end{array}$ \\
\hline $\begin{array}{l}\text { Widerstrom- } \\
\text { Noga } \\
1999\end{array}$ & 430 & & 50 & M & 77 & & 8.1 & 65 & 35 & 56 & 12 & 100 & $\begin{array}{l}\text { Pain (rehabilitation } \\
\text { admission) }\end{array}$ \\
\hline
\end{tabular}




\begin{tabular}{|c|c|c|c|c|c|c|c|c|c|c|c|c|c|}
\hline $\begin{array}{l}\text { Article } \\
\text { (by year) }\end{array}$ & 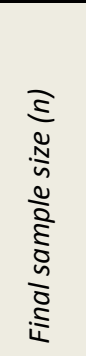 & 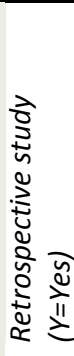 & 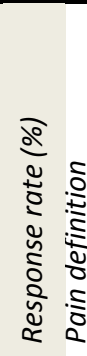 & E. & 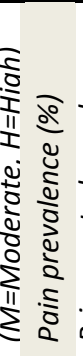 & 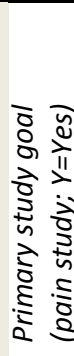 & 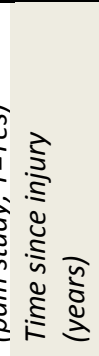 & 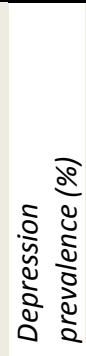 & 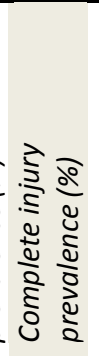 & 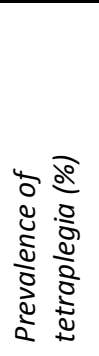 & 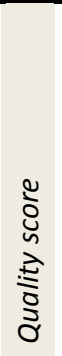 & 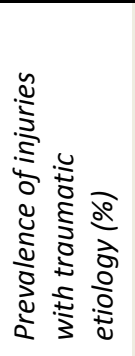 & $\begin{array}{r}\text { Pain definition } \\
\text { (patient setting) }\end{array}$ \\
\hline $\begin{array}{l}\text { Prysak } \\
2000\end{array}$ & 91 & & 50 & $M$ & 45 & & 17.5 & 22 & & 51 & 11 & & $\begin{array}{l}\text { Pain, started after } \mathrm{SCl} \text { and } \\
\text { persisted }>=4 \text { months } \\
\text { (mostly outpatient) }\end{array}$ \\
\hline $\begin{array}{c}\text { Ravenscroft } \\
2000\end{array}$ & 145 & & 67 & M & 79 & Y & 8.4 & & 50 & 43 & 12 & 86 & Chronic pain, during last year \\
\hline $\begin{array}{l}\text { Finnerup } \\
2001\end{array}$ & 330 & & 76 & & 77 & Y & 9.3 & & 48 & 36 & 14 & 75 & $\begin{array}{l}\text { Pain and unpleasant } \\
\text { sensations (outpatient) }\end{array}$ \\
\hline $\begin{array}{l}\text { Putzke } \\
2001\end{array}$ & 222 & & 100 & $\mathrm{H}$ & 44 & & & & 70 & 35 & 17 & 100 & Current pain (outpatient) \\
\hline $\begin{array}{l}\text { Turner } \\
2001\end{array}$ & 384 & & 64 & & 79 & Y & 12.2 & & 44 & 51 & 14 & 80 & $\begin{array}{l}\text { Pain causing moderate daily } \\
\text { interference during previous } \\
4 \text { weeks (mostly outpatient) }\end{array}$ \\
\hline $\begin{array}{l}\text { Klotz } \\
2002 \\
\& \text { Ville } \\
2001\end{array}$ & 1668 & & 49 & & 74 & & 12.9 & & 53 & & 12 & & $\begin{array}{l}\text { Pain (rehabilitation } \\
\text { admission) }\end{array}$ \\
\hline $\begin{array}{r}\text { McKinley } \\
2002\end{array}$ & 117 & & & M & 60 & & & 26 & 33 & 50 & 10 & 63 & $\begin{array}{l}\text { Post injury pain, requiring } \\
\text { pharmacological intervention; } \\
\text { (first rehabilitation admission) }\end{array}$ \\
\hline${ }^{\text {New }} 2002$ & 134 & Y & 100 & & 19 & & & 8 & & 33 & 13 & 0 & $\begin{array}{l}\text { Any pain, after acute phase of } \\
\text { SCI (mostly outpatient) }\end{array}$ \\
\hline $\begin{array}{l}\text { Vogel } \\
\qquad 2002\end{array}$ & 216 & & 68 & & 69 & & 14.2 & & & 57 & 13 & 91 & $\begin{array}{l}\text { Pain problem (mostly } \\
\text { outpatient) }\end{array}$ \\
\hline $\begin{array}{l}\text { Walter } \\
\qquad 2002 \\
\& \text { Walter } \\
2000\end{array}$ & 99 & & 100 & & 44 & & & 22 & & 41 & 11 & & $\begin{array}{l}\text { At least sometimes pain } \\
\text { (mostly outpatient) }\end{array}$ \\
\hline $\begin{array}{l}\text { Barrett } \\
\qquad 2003\end{array}$ & 88 & & 86 & & 75 & Y & 16.7 & & & 60 & 15 & 84 & $\begin{array}{c}\text { Pain, ache or unpleasantness } \\
>=\text { previous } 2 \text { weeks } \\
\text { Or } \\
\text { recurrent } \\
\text { during }>=4 \text { two-week periods } \\
\text { during the } \\
\text { previous year }\end{array}$ \\
\hline $\begin{array}{l}\text { Norrbrink Budh } \\
\quad 2003\end{array}$ & 456 & & 76 & M & 64 & Y & & & 32 & 46 & 15 & 75 & Pain (admission) \\
\hline $\begin{array}{l}\text { Putzke } \\
\qquad 2003\end{array}$ & 6128 & & 74 & $\mathrm{H}$ & 38 & & & & 56 & 51 & 15 & 100 & $\begin{array}{c}\text { Significant pain that } \\
\text { interfered with therapy } \\
\text { (during [first] rehabilitation } \\
\text { admission) }\end{array}$ \\
\hline $\begin{array}{l}\text { Siddall } \\
\qquad 2003\end{array}$ & 73 & & 91 & M & 81 & Y & & 25 & 38 & 49 & 16 & & $\mathrm{SCl}$ related pain \\
\hline $\begin{array}{l}\text { Yap } \\
2003\end{array}$ & 40 & & & $\mathrm{H}$ & 70 & $Y$ & & & 38 & 40 & 11 & 100 & $\begin{array}{l}\text { Pain of }>=\text { moderate daily } \\
\text { interference during previous } \\
4 \text { weeks (mostly outpatient) }\end{array}$ \\
\hline
\end{tabular}




\begin{tabular}{|c|c|c|c|c|c|c|c|c|c|c|c|c|c|}
\hline $\begin{array}{l}\text { Article } \\
\text { (by year) }\end{array}$ & 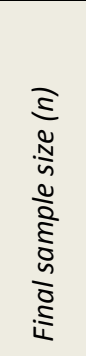 & 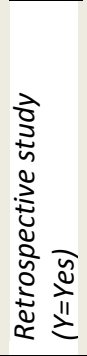 & 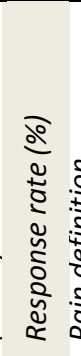 & 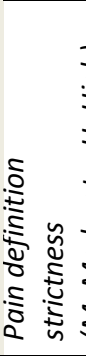 & 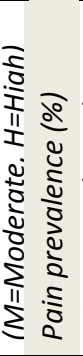 & 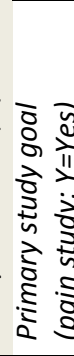 & 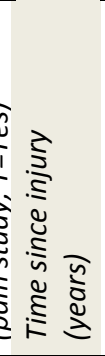 & 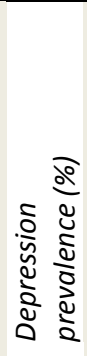 & 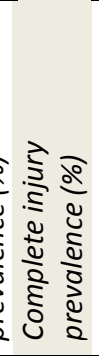 & 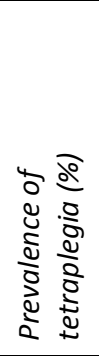 & 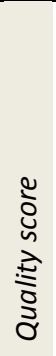 & 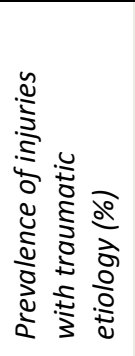 & $\begin{array}{r}\text { Pain definition } \\
\text { (patient setting) }\end{array}$ \\
\hline $\begin{array}{r}\text { Cardenas } \\
2004\end{array}$ & 2433 & & & & 81 & $Y$ & 1 & & 49 & 52 & 15 & 100 & $\begin{array}{l}\text { Not no pain during past } 4 \\
\text { weeks (mostly outpatient) }\end{array}$ \\
\hline Jan 2004 & 31 & & & & 65 & $Y$ & & & 58 & 39 & 10 & & $\begin{array}{c}\text { Pain problems during } \\
\text { previous year (mostly } \\
\text { outpatient) }\end{array}$ \\
\hline $\begin{array}{l}\text { Rintala } \\
2004\end{array}$ & 96 & & 50 & & 87 & $Y$ & 10.9 & & 44 & 50 & 15 & & $\begin{array}{c}\text { Pain (attending pediatric } \\
\text { tertiary care) }\end{array}$ \\
\hline $\begin{array}{r}\text { Saikkonen } \\
2004\end{array}$ & 76 & & 82 & $\mathrm{H}$ & 37 & & & 26 & 53 & 49 & 14 & 82 & $\begin{array}{c}\text { Pain disturbing daily life for } \\
\text { >= } 2 \text { weeks, on } 4 \text { occasions, } \\
\text { during previous year (mostly } \\
\text { outpatient) }\end{array}$ \\
\hline $\begin{array}{l}\text { Bloemen- } \\
\text { Vrencken } \\
2005\end{array}$ & 454 & & 46 & & 55 & & 13.3 & & 67 & 34 & 11 & 79 & $\begin{array}{c}\text { Pain problem during previous } \\
\text { year }\end{array}$ \\
\hline $\begin{array}{r}\text { Donnelly } \\
2005\end{array}$ & 66 & & & $M$ & 92 & $Y$ & 1 & & 55 & 55 & 13 & 100 & $\begin{array}{l}\text { Frequent pain during } \\
\text { previous half year }\end{array}$ \\
\hline $\begin{array}{l}\text { Rintala } \\
2005\end{array}$ & 348 & & 92 & $M$ & 76 & $Y$ & 17.5 & & & 46 & 17 & 78 & $\begin{array}{l}\text { Pain (at } 6 \text { months of } \\
\text { community living) }\end{array}$ \\
\hline $\begin{array}{l}\text { Meade } \\
2006\end{array}$ & 436 & & 57 & $M$ & 38 & & & & 22 & 55 & 11 & & $\begin{array}{c}\text { Chronic pain within previous } \\
\text { year }\end{array}$ \\
\hline $\begin{array}{l}\text { Jensen } \\
2007 \\
\text { \& Raichle } \\
2007 \\
\text { \& Jensen } \\
2005\end{array}$ & 147 & & 47 & & 84 & & 16.6 & & 44 & 50 & 13 & 76 & $\begin{array}{l}\text { Not no pain (8 months after } \\
\text { injury, mostly outpatient) }\end{array}$ \\
\hline $\begin{array}{r}\text { Kalpakjian } \\
2007\end{array}$ & 65 & & & $M$ & 66 & & 13.7 & & 66 & 46 & 10 & & $\begin{array}{c}\text { SCl-related pain > }=6 \text { months } \\
\text { (outpatient) }\end{array}$ \\
\hline $\begin{array}{l}\text { Pagliacci } \\
2007\end{array}$ & 403 & & 85 & $\mathrm{H}$ & 32 & & & & 50 & 37 & 15 & 100 & $\begin{array}{l}\text { Pain, excluding occasional } \\
\text { headaches or menstrual } \\
\text { cramps, >= once during } \\
\text { previous } 3 \text { months } \\
\text { (outpatient) }\end{array}$ \\
\hline $\begin{array}{l}\text { Raissi } \\
2007\end{array}$ & 54 & & 100 & & 96 & & & & & 6 & 14 & 100 & $\begin{array}{c}\text { Clinical problem: pain, during } \\
\text { previous half year (mostly } \\
\text { outpatient) }\end{array}$ \\
\hline $\begin{array}{l}\text { Wollaars } \\
2007\end{array}$ & 279 & & 49 & $M$ & 77 & $Y$ & & & 42 & 57 & 12 & 63 & $\begin{array}{l}\text { Chronic pain, >= mild or } \\
\text { infrequent problem, during } \\
\text { previous } 3 \text { months (mostly } \\
\text { outpatient) }\end{array}$ \\
\hline $\begin{array}{l}\text { Krahn } \\
2009\end{array}$ & & & & & & & & & & 56 & 14 & & $\begin{array}{c}\text { Chronic pain, >=mild problem } \\
\text { during previous year (mostly } \\
\text { outpatient) }\end{array}$ \\
\hline $\begin{array}{c}\text { Miladinovic } \\
2009\end{array}$ & 100 & & & $M$ & 50 & $Y$ & & & & 0 & 9 & & $\begin{array}{c}\text { Pain (start rehabilitation } \\
\text { admission) }\end{array}$ \\
\hline
\end{tabular}




\begin{tabular}{|c|c|c|c|c|c|c|c|c|c|c|c|c|c|}
\hline $\begin{array}{l}\text { Article } \\
\text { (by year) }\end{array}$ & 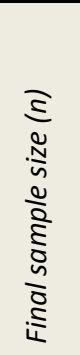 & 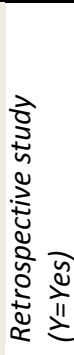 & 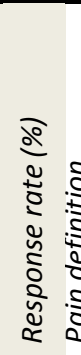 & 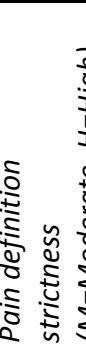 & 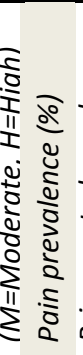 & 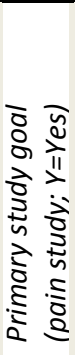 & 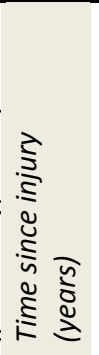 & 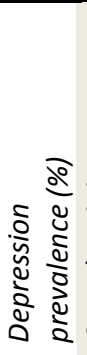 & 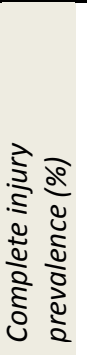 & 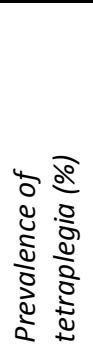 & 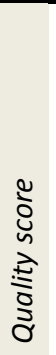 & 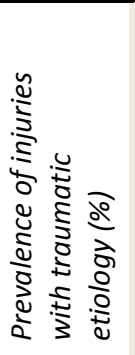 & $\begin{array}{r}\text { Pain definition } \\
\text { (patient setting) }\end{array}$ \\
\hline $\begin{array}{l}\text { Nicholson Perry } \\
2009\end{array}$ & 47 & & 45 & & 77 & & & 29 & 45 & 49 & 14 & & $\begin{array}{c}\text { Pain (start rehabilitation } \\
\text { admission) }\end{array}$ \\
\hline $\begin{array}{l}\text { Gupta } \\
\qquad 2010\end{array}$ & 276 & & 46 & $M$ & 57 & $Y$ & & & & 0 & 12 & 89 & $\begin{array}{l}\text { Bothersome, frequently } \\
\text { occurring, or periodic pain } \\
\text { (mostly outpatient) }\end{array}$ \\
\hline $\begin{array}{l}\text { Hitzig } \\
\qquad 2010\end{array}$ & 344 & & 44 & & 58 & & 19.1 & 26 & 35 & 46 & 12 & 93 & $\begin{array}{l}\text { Chronic pain during previous } \\
\text { year (outpatient) }\end{array}$ \\
\hline $\begin{array}{l}\text { Kennedy } \\
2010\end{array}$ & 80 & & & $\mathrm{H}$ & 50 & & & & 31 & 50 & 12 & & $\begin{array}{l}\text { Total of three Numerical } \\
\text { Rating Scores scores }>=11 \text {, } \\
\text { out of } 30 \text {; in last } 24 \mathrm{~h} \text {, at its } \\
\text { worst, and at its least in } \\
\text { previous week (mostly } \\
\text { outpatient) }\end{array}$ \\
\hline $\begin{array}{l}\text { Loo } \\
2010\end{array}$ & 453 & & 45 & & 41 & & 13.3 & & 65 & 34 & 12 & 72 & $\begin{array}{l}\text { Pain, as being } 1 \text { of the most } \\
\text { important condition (max. } 5 \text { ) } \\
\text { in previous year (mostly } \\
\text { outpatient) }\end{array}$ \\
\hline $\begin{array}{l}\text { Modirian } \\
2010\end{array}$ & 1295 & & 64 & & 66 & $Y$ & 14 & & 99 & 9 & 7 & 100 & $\begin{array}{c}>=\text { mild pain (mostly } \\
\text { outpatient) }\end{array}$ \\
\hline $\begin{array}{l}\text { Heutink } \\
2011\end{array}$ & 279 & & 49 & $M$ & 77 & $Y$ & & & & & 12 & & $\begin{array}{l}\text { Any pain (of either } \\
\text { musculoskeletal, spasm, } \\
\text { visceral, or neuropathic } \\
\text { origin), for more than } 6 \\
\text { months duration (outpatient) }\end{array}$ \\
\hline $\begin{array}{c}\text { Machacova } \\
2011\end{array}$ & 140 & & & $\mathrm{H}$ & 36 & & 10.5 & & & 44 & 8 & 100 & $\begin{array}{c}\text { Currently seeing a doctor for } \\
\text { pain (outpatient) }\end{array}$ \\
\hline $\begin{array}{r}\text { McKinley } \\
2011\end{array}$ & 30 & & 100 & & 67 & & & & 30 & 13 & 15 & 0 & $\begin{array}{l}\text { Pain (first rehabilitation } \\
\text { admission) }\end{array}$ \\
\hline${ }^{\text {New }} 2012$ & 45 & $\mathrm{Y}$ & 100 & & 77 & & & & 25 & 7 & 13 & 0 & $\begin{array}{l}\text { Pain which received } \\
\text { treatment or interfered with } \\
\text { function during admission } \\
\text { (rehabilitation admission) }\end{array}$ \\
\hline $\begin{array}{r}\text { Robertson } \\
2012\end{array}$ & 115 & Y & 58 & & 32 & & & & 23 & & 11 & 0 & $\begin{array}{l}\text { Pain on initial examination } \\
\text { (rehabilitation admission) }\end{array}$ \\
\hline Tan 2012 & 108 & & 100 & $M$ & 52 & & & & 55 & 91 & 13 & 0 & $\begin{array}{l}\text { Pain which received } \\
\text { treatment (rehabilitation } \\
\text { admission) }\end{array}$ \\
\hline $\begin{array}{l}\text { Ullrich } \\
\qquad 2012\end{array}$ & 87 & $Y$ & 62 & $M$ & 55 & & & & 52 & 37 & 13 & 93 & $\begin{array}{c}>=4, \text { out of } 10, \text { on Numerical } \\
\text { Rating Scale (rehabilitation } \\
\text { admission) }\end{array}$ \\
\hline
\end{tabular}


$\stackrel{n}{n}$ 


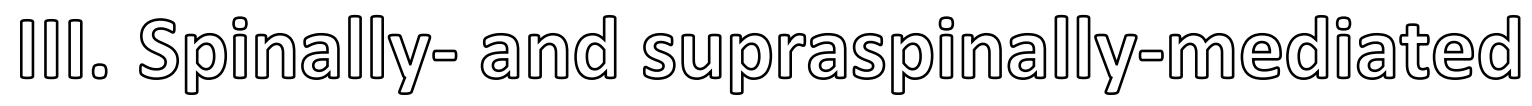 på̊n-líke response thresholds i̊n inhe

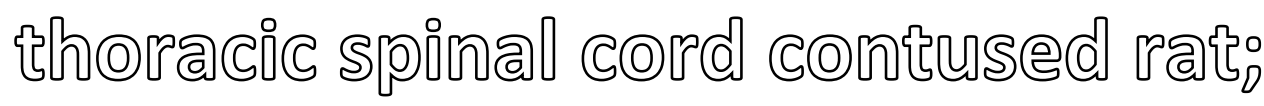

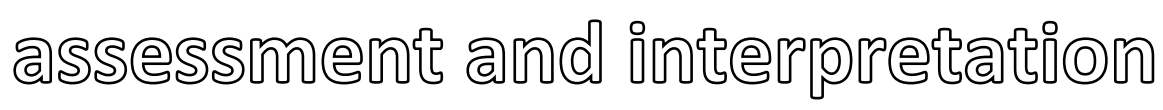




\title{
Translation of the rat thoracic contusion model; part 1 - supraspinally versus spinally-mediated pain-like responses and spasticity
}

Sebastiaan van Gorp; Ronald Deumens; Marjolein Leerink; Sandee Nguyen;

Elbert A. Joosten; Martin Marsala

\begin{abstract}
Below-level sensitivity assessment in animal models of spinal cord injury is complex. Stimulusevoked paw withdrawals can be mediated by below-level spinal cord reflexes. This makes interpretation of changes in below-level paw withdrawal response thresholds difficult, requiring both re-interpretation of prior results and novel behavioral sensory assessment methods. We here introduce a combinational-assessment design which uses the classical and (supraspinally mediated) escape-response tests for the assessment of pain-like sensitivity thresholds in acute and chronic stages of rat thoracic spinal cord contusion injury $(\mathrm{SCl})$ model. As increased hindpaw withdrawal responses after $\mathrm{SCl}$ correlate better with the spastic syndrome (not neuropathic pain), the correlation between withdrawal and spasticity response was also investigated. The escape response test can be used to assess SCl-induced changes in below-level sensitivity thresholds. These sensitivity thresholds were found to increase soon after moderate or severe $\mathrm{SCl}$, while, in parallel, hindpaw withdrawal thresholds decreased. However, the latter did not co-occur with spasticity, suggesting that $\mathrm{SCl}$-induced increased withdrawal responses are probably best interpreted as a form of hyperreflexia with pathophysiological analogies of spasms and/or clonus, or a species-specific phenomenon. Decreased below-level withdrawal thresholds do not reflect pain-like hypersensitivity in rodent models of (thoracic) $\mathrm{SCl}$, as hypoesthesia actually is found using our escape-response test. Paw withdrawals are inadequate to model pain, or spasticity, but could prove useful to model spasms or clonus, which are also significant clinical problems.
\end{abstract}




\section{Introduction}

Central neuropathic pain represents a severe, difficult to treat, and poorly understood aspect of $\mathrm{SCl}$, which, in addition, is complex to model and evaluate in animal models. In animal SCl models, pain is investigated by determining hypersensitivity, often in dermatomes below the level of injury (belowlevel). Classically, a decreased hind paw withdrawal threshold to stimuli is observed and considered to reflect a central neuropathic below-level pain state. However, a reduction in the threshold for below-level withdrawal responses (BL-WRs) can present itself without any associated changes in escape/avoidance behavior to those same stimuli (Baastrup 2010b). Reduced paw withdrawal thresholds does thus not necessarily implicate an increased aversion to the stimulus. Furthermore, much reduced brain activity evoked by electrical stimulation of skin corresponding to below-level dermatomes in similar (but anesthetized) $\mathrm{SCl}$ rats has been reported, which actually implicates a state of hypoesthesia, not hyperesthesia (Hofstetter 2003). Hence, BL-WRs are probably due to autonomic spinal hyperreflexia and one might even not be perceived/conscious at all. Also in human $\mathrm{SCl}$ patients below-level hypoesthesia is more the rule than the exception, even in patients with below-level central neuropathic pain (Celik 2012, Finnerup 2003c). Also, below-level pain in human $\mathrm{SCl}$ patients typically has a relative late onset, up to months, even years, after injury (Siddall 2003).

Therefore, we here test combination of existing paradigms in which sensitivity thresholds for belowlevel evoked thermal and mechanical stimuli are not assessed with BL-WRs, but with supra-spinally mediated Above-Level Escape Responses (AL-ERs) (Baastrup 2010b, Chaplan 1994, Detloff 2008, Vierck 1995), and see how they relate to the development of BL-WRs. AL-ER thresholds or latencies are defined as force (mechanical) or time (heat) required to elicit 1) escape or struggling behavior, or 2) vocalization. The possibility to behaviorally quantify below-level sensitivity not only allows for a better interpretation of (sensory-feedback-dependent) motor tests, but also allows evaluations of therapy efficacy on sensory function, including hypesthesia and hypersensitivity.

Furthermore, it is important to realize that $B L-W R s$ have now been postulated to represent mechanisms related to the spastic syndrome (instead of central neuropathic pain) (Baastrup 2010b). This spastic syndrome does not occur as evident in most $\mathrm{SCl}$ rats (including $\mathrm{SCl}$ rats) as it does in humans patients, in whom the most evident feature of the spastic syndrome often is the increased muscle resistance/activity (i.e. spasticity). However, spasticity in its most common and narrow definition, is defined as a velocity-dependent increase in muscle activity after tonic passive stretch (stretch hyperreflexia). Thus, such spasticity (and other reflex-like features of the spastic syndrome, such as spasms or clonus) can be easily overlooked in rats, as increases in spastic syndrome related muscle activity only needs to occur, or becomes more evident, after being evoked (e.g. by stretch). 
Therefore, in the present study we also investigate whether spasticity, defined as stretch/proprioceptive hyperreflexia (Marsala 2005), coincides with the nociceptive hyperreflexia seen with $\mathrm{BL}-\mathrm{WRs}$ in $\mathrm{SCl}$ rats.

\section{Animals, methods, and materials}

\section{Animals and surgeries}

All animal studies were approved by the University of California, San Diego institutional animal care and use committee. Twenty-two 12 weeks old Female Sprague-Dawley rats were anesthetized with isoflurane ( $5 \%$ induction, $2 \%$ maintenance; room air), placed on a Stereotaxic frame (Stoelting, Cat\# 51600 with Cat\# 51695, Wood Dale, IL, USA), and maintained at a core temperature of $37 \pm 0.3^{\circ} \mathrm{C}$ using a heating blanket. A Th9 laminectomy was performed using a dental drill. The MASCIS/NYU apparatus (WM Keck Center for Collaborative Neuroscience, Rutgers University, Piscataway, NJ, USA) was used for $\mathrm{SCl}$. The laminectomy site was filled with mineral oil in which the tip of a small thermocouple (Physitemp, Cat\# IT-14, Clifton, NJ, USA) was submerged and used to manually keep the spinal cord at $37 \pm 0.3^{\circ} \mathrm{C}$ with warmth from surgical lights (Fiber-Lite, Cat\# MI-150 \& BGG1823M, Dolan-Jenner, Boxborough, MA, USA). Next, oil and lamps were removed and the rod dropped using a height of 6.25 (mild $\mathrm{SCl}, \mathrm{n}=7$ ), 12.5 (moderate $\mathrm{SCl}, \mathrm{n}=5$ ), or $25 \mathrm{~mm}$ (severe $\mathrm{SCl}, \mathrm{n}=5$ ). Five sham operated animals were included.

Group designation was not known by the experimenters doing behavioral testing. SvG was involved in all behavioral testing, ML, and SN were involved in Basso, Beattie, Bresnahan (BBB) scoring.

\section{Post-surgical care}

Buprenorphine $(0.05 \mathrm{mg} / \mathrm{kg}$, s.c., Reckitt Benckiser, Richmond, VA, USA), $5 \mathrm{~mL}$ of Lactated ringer, and $10 \mathrm{mg} / \mathrm{kg}$ of Cefazolin (Novaplus/Sandoz, Holzkirchen, Germany) was given after surgery. Bladders were emptied manually twice daily. Sulfamethoxazole and Trimethoprim USP (200mg \& 40mg per $250 \mathrm{~mL}$ drinking water, Hi-Tech Pharmacal, Amityville, NY, USA) was given for at least 10-14 days or until autonomic bladder voiding occurred. Any ill-appearing animals received additional days of above-mentioned antibiotic treatments.

\section{Open field locomotion testing}

Locomotion recovery after $\mathrm{SCl}$ was monitored using a rating scale based on the BBB open field locomotor rating scale (0 to 21), as described before (Basso 1995, Basso 1996). 


\section{Sensory testing}

Hind paw withdrawal response thresholds (dorsally applied von Frey filaments; see below, this section) and supraspinally mediated 'above-level'-escape response (AL-ER) thresholds were assessed. An AL-ER is defined as an escape-attempt from the investigator's loose, but fully supportive, grip (Fig.1) and has to include the elaborate use of their front paws or a vocalization. The applied stimuli were either a gradually increasing force to the hind paws (using the Analgesy-Meter; Cat\# 37215, Ugo-Basile, Collegeville, PA, USA), or a constant heat stimulus (intensity 17, cut-off at 30 sec) to the hind paws (using an constant infrared heat source; Cat\# 37360, Ugo-Basile, Collegeville,

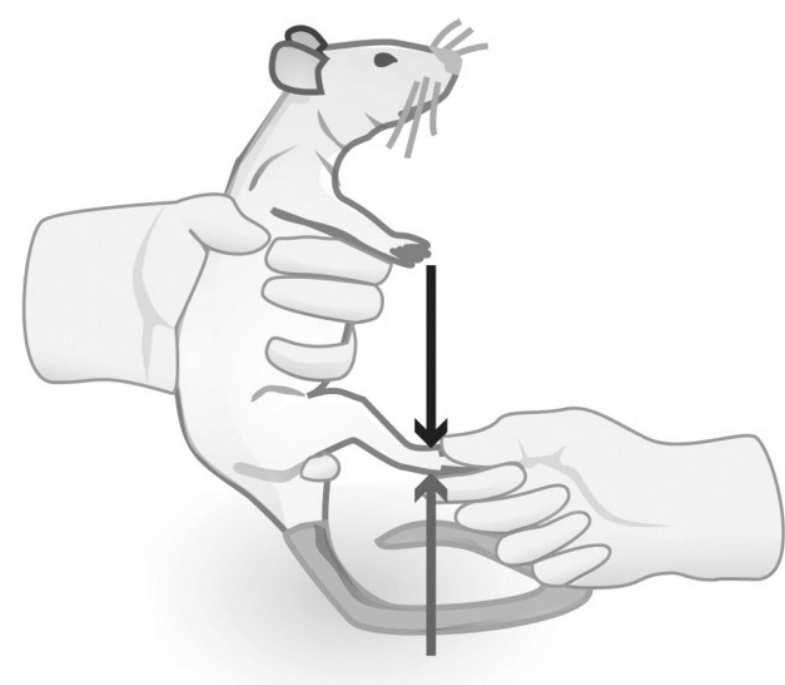

Figure III-1: Schematic of Above-Level Escape Response testing upon below-level stimulation. Shown is the way the animals were held for the AL-ER testing upon hindpaw stimulation with a mechanical (black arrow, dorsal) or a thermal (grey arrow, plantar) stimulus. The animals were loosely held so that animals could escape from the investigator's grip. An Above-Level Escape Response refers to escape behavior elicited by structures innervated above the level of injury used. Here we used a thoracic injury; hence, AL-ERs were defined as the transition of a tranquil state to a state of fleeing/distress with respect to activation of the frontlimbs, and shoulder-/neck-/head-area, and/or vocalization. Per time point, the hindpaws were alternatingly tested with an interval of 1-2hours, up to a total of four times per paw for each stimulus.
PA, USA). The hindpaw tested was gently restrained by the investigator to prevent reflex-related paw withdrawal (Fig.III-1).

For the AL-ER tests, both hind paws were tested four times, alternately, for each test, with a testing interval of 1-2 hours and maximal four measurements per day. Maximum cut-off values for the stimuli or latency were at approximately two times that of response threshold of uninjured animals to prevent tissue damage. Prior to (7 days) and during the entire experimental period ( 5 days a week), the animals were extensively habituated to the experimenter in such a way that the animals could be held upright (loosely) during all sensory assessments. Habituation consisted of picking up the animal and holding/handling it twice daily for 3-5 minutes. During habituation the animals were presented to the test setup, as if they were tested, but without the application of mechanical or thermal stimuli (the testing devices were switched on/operated during habituation, to acclimatize the animals to any noise of 
each device when operated).

To determine the hind paw withdrawal thresholds (von Frey monofilaments, up-down method (Chaplan 1994)) the animals were also loosely held upright, to circumvent the requirement of weight support (Detloff 2008).

\section{Assessment of stretch induced muscle resistance}

Peripheral muscle resistance was measured using a previously described spasticity-meter (Marsala 2005). Briefly, animals were placed in a restrainer and a hind paw was taped to a metal plate driven by a computer-controlled stepper motor. The resistance of the ankle to dorsiflexion was measured during stepper motor-driven ankle flexion $\left(40^{\circ}\right)$, and always in conjunction with the EMG of the ipsilateral m.gastrocnemius (described below, this section). Resistance was measured in awake animals using medium, fast and slow ankle rotation speeds (i.e. $40^{\circ}$ in $0.5,0.25$ and $1 \mathrm{sec}$, respectively). Consecutively, the muscle resistance was measured in anesthetized animals (at medium speed), to identify the mechanical (vs. the neurogenic, isoflurane-sensitive) component of measured ankle resistance. Hence, active muscle resistance could be computed (resistance when awake minus resistance when anesthetized). Also, spastic components of muscle resistance could be detected, as spasticity is velocity-dependent. To record EMG activity, a pair of tungsten electrodes was inserted percutaneously into the gastrocnemius muscle $1 \mathrm{~cm}$ apart. EMG signals were bandpass filtered (100 Hz to $10 \mathrm{kHz}$ ) and recorded before, during, and after ankle dorsiflexion. Only EMG data from the fast ankle rotation measurements was used for the detection of rotation-induced EMG amplitude increases. Average EMG amplitudes were calculated for the two seconds before ankle rotation, and a period of 0.20 seconds in which the maximal ankle rotation was achieved. Each recorded value was the average of three repetitions.

\section{Statistical analyses}

Results were analyzed using ANOVA (one-way, or two-way group $\times$ time repeated measures, using a fixed-effect model), with a Bonferroni post hoc test for multiple comparisons (GraphPad Prism, La Jolla, CA, USA). Unequal variances were not observed. Results are analyzed as two-tailed and expressed with standard error of the mean (SEM), unless specified otherwise. A P-value of 0.05 was considered significant.

We certify that all applicable institutional and governmental regulations concerning the ethical use of animals were followed during the course of this research. 


\section{Results}

A) BBB Locomotor scores

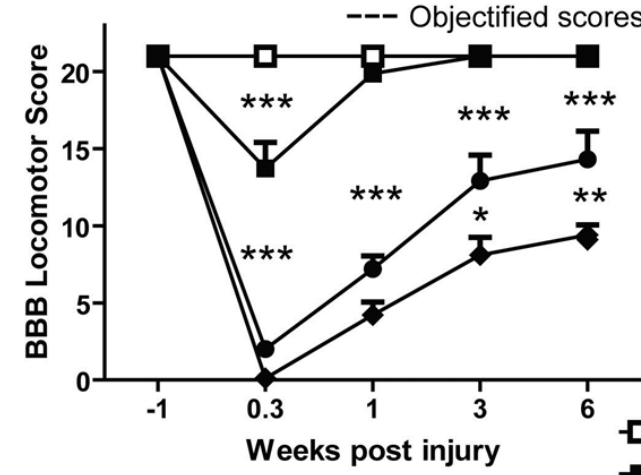

- $\square$ Sham

- Mild SCl

Moderate SCl

Severe SCI
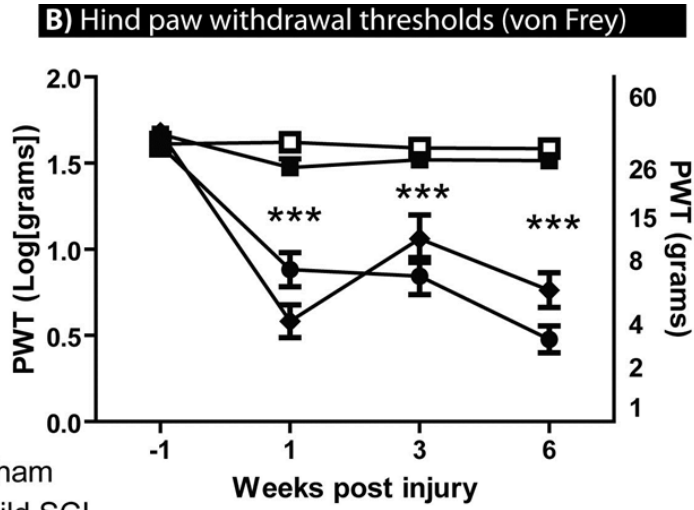

D) Hind paw evoked AL-ERs (Thermal stimulus)
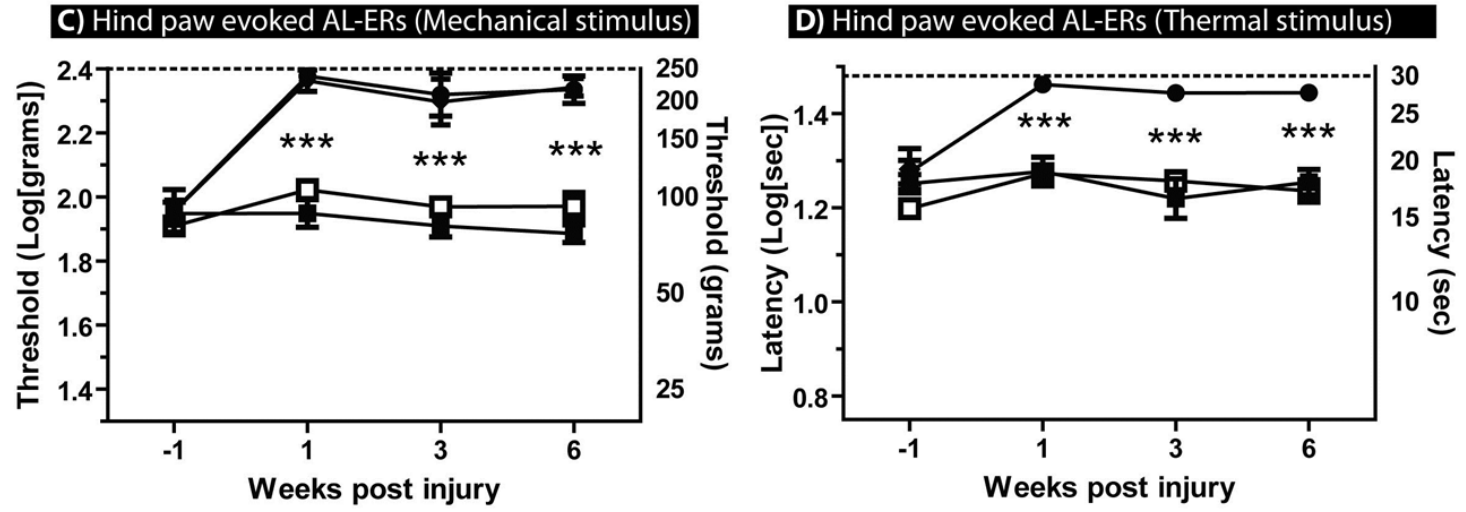

E) Active muscle resistance upon ankle rotation

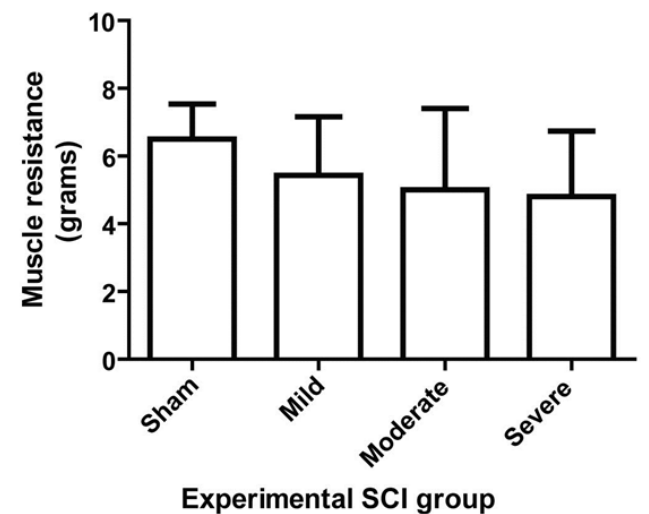

Figure III-2: Spinal and supraspinal pain-like responses and spasticity after thoracic SCI. A) Shown are the typial open-field BBB locomotor scores of the sham, mild, moderate, and severe $\mathrm{SCl}$ groups. B) Using the up-down method to the dorsal aspect of the hindpaws we confirmed that below-level withdrawal responses decrease after $\mathrm{SCl}$ animals. Compared to sham-operated animals only more than mild $\mathrm{SCl}$ animals showed significantly decreased hindpaw withdrawal thresholds (Repeated Measures ANOVAs; P<0.001). C-D) In contrast, pain-perception/AL-ER thresholds to below-level mechanical (C) and thermal (D) stimuli increased in animals with a more than mild SCI (Repeated Measures ANOVAs; $\mathrm{P}<0.001$ ). E) The occurrence of belowlevel paw withdrawal hyperreflexia did not coincide with stretch-evoked muscle activity, as no increases in (active) muscle resistance were noted in any $\mathrm{SCl}$ group up to the end of the study (i.e. six weeks post injury). 
SCls induced with the MASCIS/NYU apparatus using $6.25 \mathrm{~mm}, 12.5 \mathrm{~mm}$, and $25 \mathrm{~mm}$ impact/drop heights, resulted in significantly different injury severities, as assessed with the BBB-locomotor score (Variation between any two experimental groups; Bonferroni; P $\leq 0.04$; Fig.2A). Trends in BBB-scores are similar to the ones reported originally, indicating a similar extent of the injuries (Basso 1995, Basso 1996). Weight-support (i.e. BBB score $\geq 9$ ) was absent in moderate $\mathrm{SCl}$ animals prior to the 3 weeks assessment and remained absent in most severe $\mathrm{SCl}$ animals.

Graded SCCI resulted in a decreased hind paw withdrawal threshold in the moderate and severely injured groups (i.e. 12.5 and $25 \mathrm{~mm}$ weight drop groups), when compared to the sham-operated group (Repeated Measures ANOVA; $\mathrm{P}<0.0001$; Fig.2B). However, mild injury $(6.25 \mathrm{~mm}$ ) did not give significant threshold changes in comparison to sham operated animals $(P=0.21)$. No significant differences were present between moderate and severe $\mathrm{SCl}$.

Using supraspinally mediated above-level escape responses (AL-ERs), the below-level sensitivity thresholds were assessed. AL-ER thresholds for mechanical stimuli changed only for moderate and severe $\mathrm{SCl}$, when compared to sham-operated animals (Repeated Measures ANOVA; $\mathrm{P}<0.001$; Fig. III-2C). Testing was somewhat difficult due to pronounced bilateral and complex hind limb motor activity (i.e. jumping/kicking-like behavior), but AL-ER thresholds after moderate $\mathrm{SCl}$ were noted to increase for thermal stimuli ( $\mathrm{P}<0.001$; Fig. III-2D), yet it was impossible to confidently assess thermal AL-ER thresholds in the severe $\mathrm{SCl}$ rats. The complex motor response to stimuli was present even in the acute $\mathrm{SCl}$ phase, in which stepping was absent during open-field testing.

Analysis of active muscle resistance in the gastrocnemius muscle upon ankle rotations to assess stretch hyperreflexia, a measure of spasticity, showed no difference between experimental groups at any rotation speed (One-way ANOVAs; $P \geq 0.63$ ) nor time point measured (i.e. one, three, and six weeks after injury; $\mathrm{P}=0.94, \mathrm{P}=0.92$, and $\mathrm{P}=0.88$, respectively; Fig. III-2E shows active muscle resistance at six weeks). Also, ankle rotations m.gastrocnemius EMG amplitudes did not increase in any of the experimental groups, when compared to their resting stages (relative values; fastest ankle rotation speed: sham, mild, moderate, and severe SCl groups; Student's t-tests; $\mathrm{P}=0.75, \mathrm{P}=0.82$, $\mathrm{P}=0.48$, and $\mathrm{P}=0.25$, respectively).

\section{Discussion}

Here we show that thresholds for below-level sensitivity increase after more than mild SCl (assessed with AL-ER thresholds for both thermal and mechanical stimuli) during the first six weeks after $\mathrm{SCl}$, which makes it a useful test for SCl therapy evaluations. In contrast, BL-WR thresholds decreased in those same animals, indicating a limited correlation of BL-WR with pain perception. Furthermore, 
the AL-ER test revealed forceful bilateral hindpaw motor ability (kicking/jumping) upon modest sensory stimulation of a single hindpaw, even when $\mathrm{SCl}$ animals were still unable to support their bodyweight during locomotion. The latter is in line with observations that complex BL-WR can also be evoked in complete spinal cord transected animals, even within hours after injury (Schouenborg 1992).

Importantly, although AL-ERs are supraspinally mediated (strictly speaking they are above-level mediated), they still should be carefully interpreted as responses to pain, as AL-ERs reflect supraspinal responses which do not necessarily require cortical processing and could thus merely be brainstem mediated reflexes (Vierck 2006).

The observed nociception-evoked hyperreflexia is different from spasticity or rigidity, as it did not coincide with increased (stretch-evoked) muscle activity. Nonetheless, both the problems arising from the spastic syndrome in humans and the decreased BL-WR thresholds in $\mathrm{SCl}$ rats respond to baclofen ( $\mathrm{GABA}_{B}$ agonist) treatment, while central neuropathic pain in humans hardly does, if at all (Teasell 2010). Nevertheless, the spastic syndrome consists of an array of only partly overlapping 'positive signs' of the Upper Motor Neuron syndrome; which includes not only spasticity, but also spasms, pathological reflexes, clonus, hypertonia, and dystonia (whereas its 'negative signs' relate to the loss of motor control). The hindpaw hyperreflexia as seen in the current study/model (i.e. complex hindpaw motor activity upon sensory stimulation in paraplegic rats) could still correspond well to the spastic syndrome, as observed in human SCl patients, as it shows similarities to, for instance, spasms or clonus. The latter has similarities with the withdrawal responses in terms of the rhythmicity and a fading ending, although clonus is classically elicited through stretch receptors.

When compared to humans (Jacobs 1995), it is remarkable that rats with moderate (or even severe) $\mathrm{SCl}$ show a high degree of motor function as well as complex below-level paw withdrawal behavior, but still have poor below-level perception. In addition, rat SCl result in much fewer cases of spasticity and less pronounced spinal shock phases (Akhtar 2008, Bennett 1999). Apparently, inter-species differences in the degree of spinal autonomy (supra-spinal involvement) for locomotion function do exist. This suggests a strong complex autonomic sensorimotor function of the rodent spinal cord, which will be mostly attributed to the relative large role of the Central Pattern Generator (CPG) in the locomotion of quadrupeds (see our other manuscript) (Barriere 2008, Magnuson 2005). In summary, nociception-evoked hindpaw hyperreflexia as observed in $\mathrm{SCl}$ rats still has, on the one hand, a debatable clinical relevance as it doesn't correlate with hypersensitivity and could be solely due to species specific properties of spinal function, but, on the other hand, resembles aspects of spasms and/or clonus, as seen in human $\mathrm{SCl}$ patients. 
$\stackrel{f}{\risingdotseq}$ 


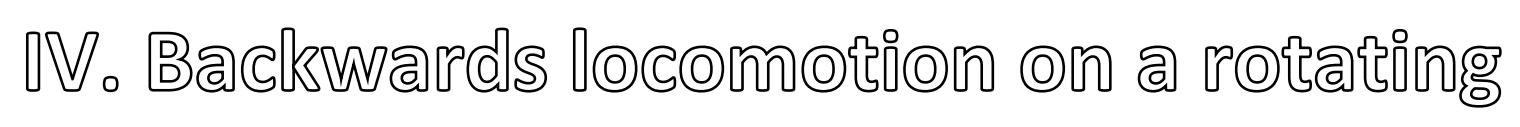
rođd: a high

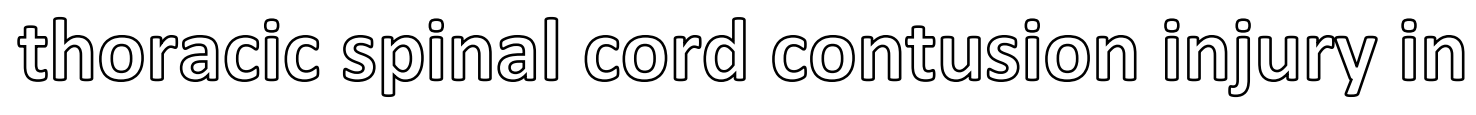
『ats? 


\title{
Translation of the rat thoracic contusion model; part 2 - forward versus backwards locomotion testing
}

\author{
Sebastiaan van Gorp; Marjolein Leerink; Sandee Nguyen; Oleksandr Platoshyn; \\ Martin Marsala; Elbert A. Joosten
}

\begin{abstract}
Locomotion analyses in rat spinal cord contusion injury (SCl) models are widely used for the evaluation of recovery of supraspinal locomotor control. However, much locomotor recovery recorded with commonly used tests are independent of below-level perception, highly mediated through autonomic propriospinal circuitry, and thus lack sensitivity for spinal cord integrity. Here we report a behavioral motor test which is more sensitive for spinal cord integrity and/or the disability in the chronic phase after injury: the backwards locomotion rotating rod. A standard rat rotating rod test was run in reverse while modified to accommodate $\mathrm{SCl}$ rats. The rod diameter was increased and a thin rubber lining was added. As a reference, we included commonly used motor tests: BBB locomotor scoring, Catwalk gait analysis, Motor Evoked Potentials, and the Single Frame Analysis, but also a forward rotating rod test and the $55^{\circ}$ Inclined Ladder Test. Unlike commonly used motor test, the backwards locomotion rotating rod test does significantly discriminate both sham-operated vs. mild $\mathrm{SCl}$ animals, and mild vs. moderate $\mathrm{SCl}$ animals at three and six weeks post injury. Moderate $\mathrm{SCl}$ animals were virtually unable to make even the slightest backwards hind paw movement. The backwards locomotion ability in the chronic post injury phase correlate best with acute BBB locomotor scores. Our data shows that backwards locomotion is a highly sensitive test to assess spinal cord injury and its integrity after rat $\mathrm{SCl}$. Backwards locomotion testing may enhance translatability of experimental results into the clinic.
\end{abstract}




\section{Introduction}

The application of animal models in spinal cord injury $(\mathrm{SCl})$ research is complicated due to difficulties in sensitive and specific quantification of locomotor outcome, though behavioral outcomes are the most important argument for using animal models. Locomotor outcomes are often assumed to correlate well with $\mathrm{SCl}$ regeneration (long-tract integrity), but this is a feature which might be poorly present in widely used animals models. Behaviorally, in the chronic phase after $\mathrm{SCl}$ in rodents, i.e. only a few weeks after injury, most mildly injured $\mathrm{SCl}$ animals have spontaneously recovered to be indistinguishable from sham-operated animals, although only half of the spinal cord is spared (Basso 1996). Furthermore, moderate SCI animals generally show merely a few mild deficits, and even severely injured $\mathrm{SCl}$ animals (reported to have even $<10 \%$ spared spinal cord tissue) regain weight supported stepping (Basso 1996). Also, recovery of below-level sensory perception seems barely, if at all, required for regaining basic quadruped locomotion (see the accompanying manuscript and Hofstetter et al. (Hofstetter 2003)). Hence, the question is: if current behavioral tests have difficulties to detect even such fairly large disruptions in the spinal cord, how reliable are these tests for (not) measuring therapeutic effects?

Agreed, the animal spinal cord (below the level of injury) is since long known to be able to implement/recover much basic motor functionality autonomously by strengthening preexisting propriospinal circuitries and, in particular the central pattern generator (see our other manuscript, Barriere et al, and Tillakaratne et al. (Barriere 2008, Tillakaratne 2010)). This notion is further supported by the fact that even after full spinal cord transection, but only when performed after a prior contusive $\mathrm{SCl}$, some recovery in hindlimb function is still noted (Basso 1996). However, locomotion recovery through autonomic spinal cord circuitry can be expected to be of much less relevance in the human condition. This is due to the higher degree of instability of the basic posture during human bipedalism which would logically require more advanced (read: supraspinal) modulation. In this perspective it is not surprising that, in contrast to quadrupeds, I) adequate below-level sensory perception after $\mathrm{SCl}$ in humans has proved to be a good predictor for locomotor recovery and II) it takes a human relatively long to learn how to walk as a child. Hence, basic locomotor tests in $\mathrm{SCl}$ models not only seem to lack desirable sensitivity for $\mathrm{SCl}$ injury, but it also (or therefore) seems not to be sufficiently specific for the human $\mathrm{SCl}$ condition.

Subsequently, we focused on motor tests which (on theoretical grounds) might be more sensitive for spinal cord integrity through the requirement of supraspinal control (i.e. requiring more advanced sensorimotor modulation). Hence, the climbing test ( $55^{\circ}$ Inclined Ladder Test) and the rotating rod test were included and tested for their sensitivity for mild and moderate thoracic spinal cord 
contusion injury in rats, a model widely used and with reproducible results. It is known (from their use in other $\mathrm{SCl}$ models) that more severely injured animals barely, if at all, recover function as assessed with these test, which is suggestive for a relatively high requirement of supraspinal motor control (Hamm 1994, Semler 2011, Sheng 2004, Simard 2012). In order to further increase the requirement of supraspinal motor control on locomotion testing and decrease the impact of basic spinal autonomic locomotion circuitry, the backwards locomotion rotating rod test was developed. As a reference, severe $\mathrm{SCl}$ animals were included, Myogenic Motor Evoked Potentials (MMEPs) to transcranial electrical motor cortex stimulation were measured, and locomotion ability was scored with the widely used Basso, Beattie, Bresnahan (BBB)-locomotor scoring method (Basso 1995, Basso 1996). To objectify some of the key locomotor features of the BBB-score, the single frame analysis (Rump Height Index and Foot Stepping Angle) and Catwalk gait analysis (Regularity Index) were used (Koopmans 2005, Semler 2011).

\section{Animals, methods, and material}

\section{Animals and surgeries}

All animal studies were approved by the University of California, San Diego institutional animal care and use committee. Twenty-two 12 weeks old Female Sprague-Dawley rats were anesthetized with isoflurane (5\% induction, $2 \%$ maintenance; room air), placed on a Stereotaxic frame (Stoelting, Cat\# 51600 with Cat\# 51695, Wood Dale, IL, USA), and maintained at a core temperature of $37 \pm 0.3^{\circ} \mathrm{C}$ using a heating blanket. A Th9 laminectomy was performed using a dental drill. The MASCIS/NYU apparatus (WM Keck Center for Collaborative Neuroscience, Rutgers University, Piscataway, NJ, USA) was used for $\mathrm{SCl}$. The laminectomy site was filled with mineral oil in which the tip of a small thermocouple (Physitemp, Cat\# IT-14, Clifton, NJ, USA) was submerged and used to manually keep the spinal cord at $37 \pm 0.3^{\circ} \mathrm{C}$ with warmth from surgical lights (Fiber-Lite, Cat\# MI-150 \& BGG1823M, Dolan-Jenner, Boxborough, MA, USA). Next, oil and lamps were removed and the rod dropped using a height of 6.25 (mild $\mathrm{SCl}, \mathrm{n}=7$ ), 12.5 (moderate $\mathrm{SCl}, \mathrm{n}=5$ ), or $25 \mathrm{~mm}$ (severe $\mathrm{SCl}, \mathrm{n}=5$ ). Five sham operated animals were included.

\section{Post-surgical care}

Buprenorphine $(0.05 \mathrm{mg} / \mathrm{kg}$, s.c., Reckitt Benckiser, Richmond, VA, USA), $5 \mathrm{~mL}$ of Lactated ringer, and $10 \mathrm{mg} / \mathrm{kg}$ of Cefazolin (Novaplus/Sandoz, Holzkirchen, Germany) was given after surgery. Bladders were emptied manually twice daily. Sulfamethoxazole and Trimethoprim USP (200mg \& 40mg per $250 \mathrm{~mL}$ drinking water, Hi-Tech Pharmacal, Amityville, NY, USA) was given for at least 10-14 days or until autonomic bladder voiding occurred. Any ill-appearing animals received additional days of above-mentioned antibiotic treatments. 


\section{Open field locomotor testing}

Locomotion recovery after $\mathrm{SCl}$ was monitored using a rating scale based on the Basso, Beattie, Bresnahan (BBB) open field locomotor rating scale (0 to 21), as described before (Basso 1995, Basso 1996).

\section{Gait analysis}

The CatWalk apparatus (7.1, Noldus, The Netherlands) was used to quantify gait parameters by semi-automated footprint analyses during runway crossings (Koopmans 2005). Animals were trained for smooth walkway crossing and animals had always access to their home cage and a treat (Certified Supreme Mini-Treats ${ }^{\mathrm{TM}}$, Cat\# F05472-1, Frenchtown, NJ, USA) at the end of the run (done for catwalk, single frame, and ladder tests).

\section{Single frame hind limb motion analysis}

Bilateral video captures allowed analysis of the foot-stepping angle (FSA) and the rump-height index (RHI), as described previously (Semler 2011). The FSA is the angle at which the hind paw is placed on the ground. The RHI was defined as the highest point of the base of the tail during a run. VirtualDub 1.9.11 (http://www.virtualdub.org) and Screen Ruler V1.0.1a (http://www.caveworks.net) were used for analyses.

\section{Integration of objective measures (Catwalk, FSA, and RHI) into BBB-locomotor score}

BBB-scores allows some experimenter subjectivity, hence we tried to objectify some of the midrange items of the BBB-score, which are regarded as key items in the recovery of SCl. First, animals were grouped in BBB-scores $\leq 7,8,9,10$, or $\geq 11$, using the Foot Stepping Angle (to define plantar stepping) and Rump Height Index (to define weight support) from the Single Frame Analysis (Semler 2011). Secondly, frequency of frontlimb-hindlimb coordination was objectively assessed using the Catwalk gait analysis by counting the coordinated runs (out of three runs) (Koopmans 2005).

\section{Inclined ladder test}

The $55^{\circ}$ Inclined Ladder Test was performed using an inclined 20-rung ladder, as previously described (Semler 2011). The correct placing of a hind paw and sustained position until its next forward move was counted over the 18 center rungs.

\section{Myogenic Motor Evoked Potentials}

Myogenic Motor Evoked Potentials (MMEPs) in the gastrocnemius muscle were measured after being elicited by transcranial electrical stimulation of the motor cortex using two percutaneously placed electrodes, as described before (van Gorp 2013). 


\section{Rotating rod and the backwards Locomotion test}

A rotating rod apparatus (Rota-Rod, Stoelting, Cat\# 52790, Wood Dale, IL, USA) was modified and used to assess motor performance (increased diameter from $33 / 4$ " to 5 " and lined with $1.5 \mathrm{~mm}$ thick rubber). Rats were placed on the elevated reversed rotating rod, which was started at $4 \mathrm{rpm}$, linearly accelerated to $40 \mathrm{rpm}$ over $60 \mathrm{sec}$, with a cut-off of 150 seconds. Maximum falling latency out of three trials was recorded. Timing started after hindpaw movement was observed. Rats were given a minimum of $30 \mathrm{~min}$. between trials. Two hand-held small transparent acrylic plates $(400 \times 50 \mathrm{~mm}$; see Fig. IV-1) and treats (as above) prevented rats from changing direction on the rotating rod and to motivate the animals for rotating rod testing.

\section{Statistical analyses}

Results were analyzed using ANOVA (one-way, or two-way group $\times$ time repeated measures, using a fixed-effect model), with a Bonferroni post hoc test for multiple comparisons (GraphPad Prism, La Jolla, CA, USA). Unequal variances were not observed. Results are analyzed as two-tailed and expressed with standard error of the mean (SEM), unless specified otherwise. A P-value of 0.05 was considered significant.

We certify that all applicable institutional and governmental regulations concerning the ethical use of animals were followed during the course of this research.

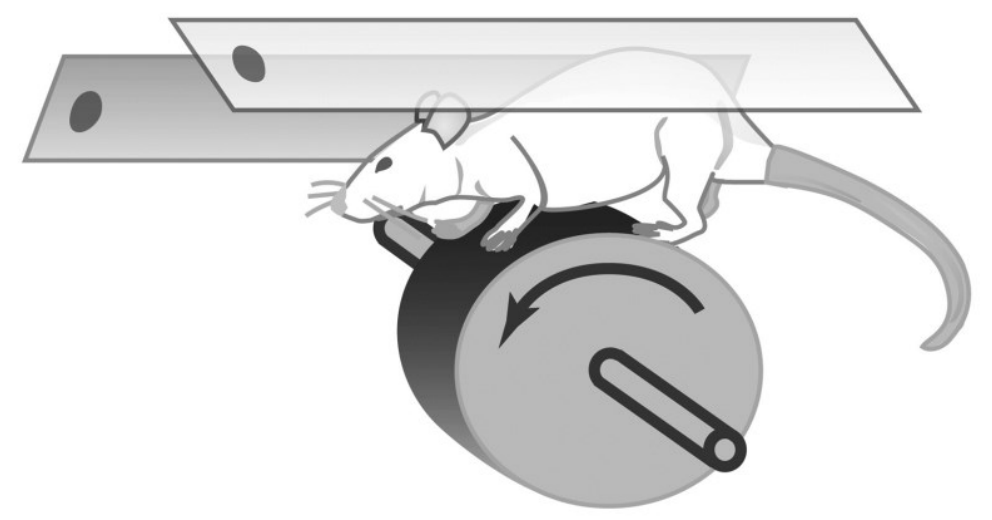

Figure IV-1: Backwards locomotion rotating rod. A rotating rod apparatus was slightly modified by increasing the rod diameter to $5 "$ and lining it with $1.5 \mathrm{~mm}$ thick rubber. Two hand-held small transparent acrylic plates $(400 \times 50 \mathrm{~mm})$ and treats prevented rats from changing direction on the rod. 


\section{Results}

All $\mathrm{SCl}$ severities caused a significant acute (three days post injury) reduction in BBB scores, compared to sham operated animals (Fig. IV-2A solid lines; Bonferroni: $\mathrm{P}<0.001$ ). Next, a partial or complete recovery in BBB scores followed (i.e. up to six weeks post injury). Notably, differences between sham and mildly injured animals merely differed in the very acute phase (Bonferroni: $\mathrm{P}<0.001)$, while differences between moderately and severely injured animals only became marginally notable after three weeks post injury (Bonferroni: $\mathrm{P}<0.05$ ). These BBB-scores are similar to the ones reported originally, using the same SCI model (Basso 1995, Basso 1996). There, most mild $\mathrm{SCl}$ animals also recovered to a $\mathrm{BBB}$ score of $\geq 20$, although a $>50 \%$ white matter loss was reported at the level of injury.

Catwalk, FSA, and RHI barely improve sensitivity for SCl compared to the BBB-locomotor score

Implementing some objective measures to the BBB-locomotor scores gave only slight, nonsignificant, changes in original BBB-scores (compare solid and dotted lines in Fig. IV-2A; Repeated Measures ANOVA per injury severity: $P \geq 0.63$ ). Individually, frontlimb-hindlimb coordination, as assessed by number of fully coordinated walkway crossings during gait analysis (coordinated crossing: a regularity index of 100\%) (Koopmans 2005), was not significantly affected in mild SCI animal when compared to sham-operated animals (Fig. IV-2B; Repeated Measures ANOVA: P=0.31). Coordination in moderate and severe $\mathrm{SCl}$ animals differed significantly from each other at three and six weeks post injury (Bonferroni: $\mathrm{P}<0.05$ ). Also, after 3 weeks Rump Height Indices only show significant disabilities for the severe $\mathrm{SCl}$ animals, when comparing with sham animals (Fig. IV-2C; Bonferroni: P $\leq 0.001)$. Last, Foot Stepping Angles were only significantly increased for severe $\mathrm{SCl}$ animals (at one and three weeks post injury; Fig. IV-2D; Bonferroni Post-Hoc: $\mathrm{P}<0.05$ ) and moderate $\mathrm{SCl}$ animals (at one week post injury; Bonferroni Post-Hoc: $\mathrm{P}<0.05$ ), when compared to either sham animals. Nonetheless, at the acute phase (one week post injury) Foot Stepping Angles, as well as Rump Height Indices, did show significant different disabilities between moderate and severe SCI animals (Bonferroni: $\mathrm{P}<0.05$ ), while BBB-scores did not. 

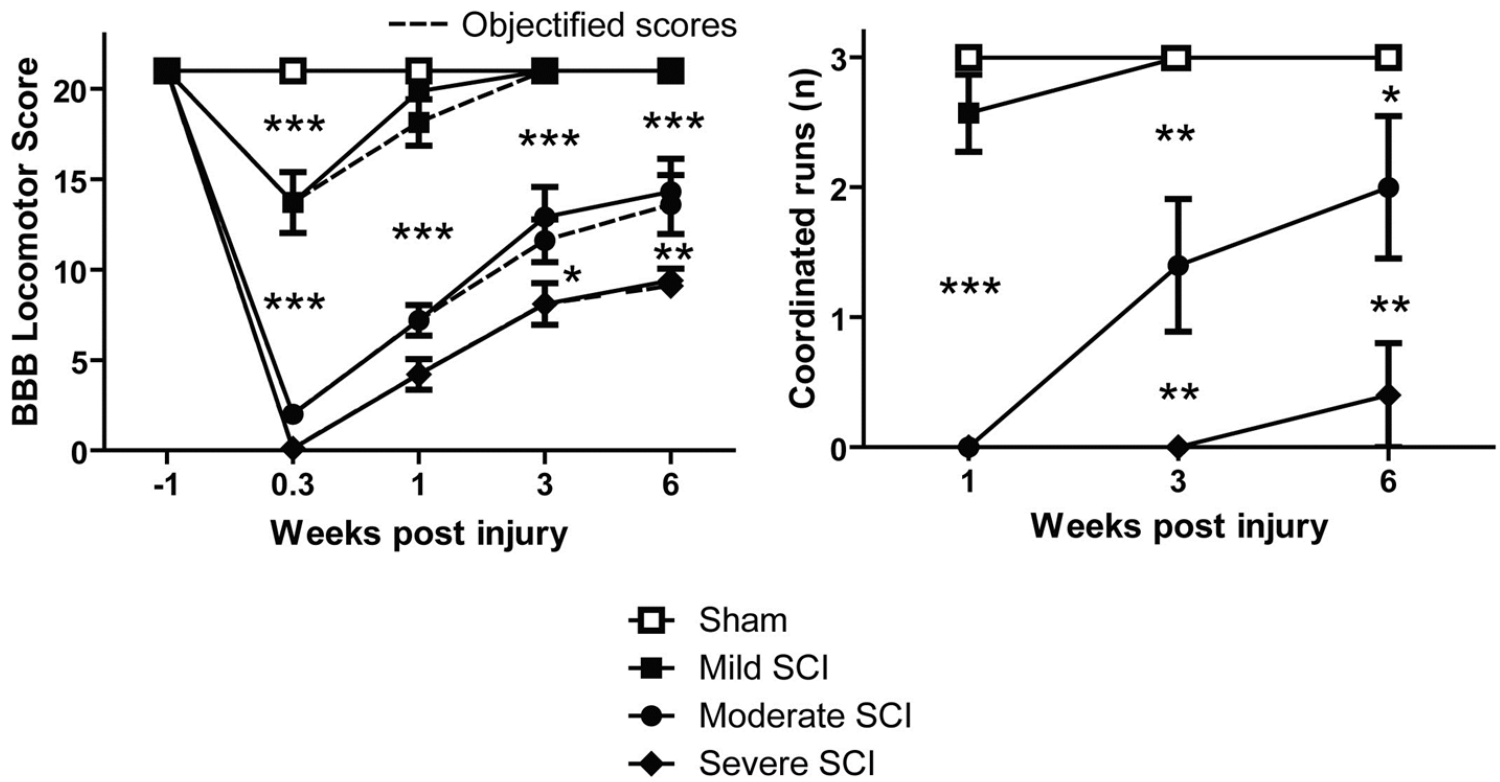

\section{C) Rump Height Index}

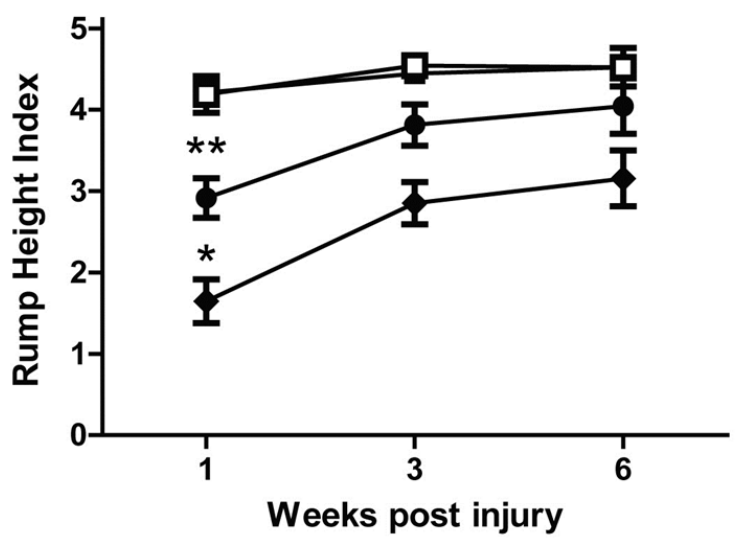

D) Foot Stepping Angle

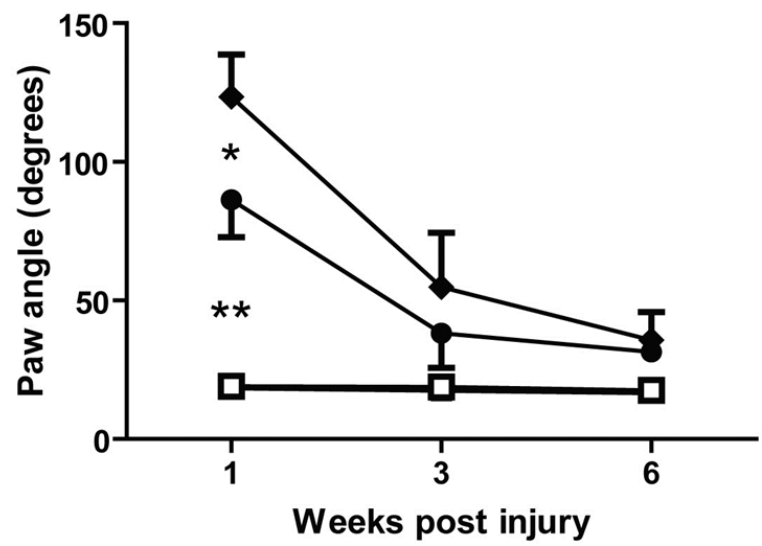

Figure IV-2: Results from locomotion tests which depend little on supraspinal locomotion control. Note that sham-operated and mild SCl rats are indistinguishable after the acute phase post SCl. A) open-field locomotion scores as assessed with the BBB locomotor scale (solid lines), and the BBB locomotor scale which was objectified for coordination, plantar stepping, and weight-support (using the Catwalk, Foot Stepping Angles, and Rump Height Index, respectively; interrupted lines). Note that even severe $\mathrm{SCl}$ animals regain weight support (scores of $\geq 9$ ). All four groups differed significantly from each other (Repeated Measures ANOVA: P $\leq 0.02$ ), but sham-operated and mild $\mathrm{SCl}$ only differed significantly at 3 days post injury (Bonferroni: $\mathrm{P} \leq 0.001$ ). B) Displayed is the number of coordinated runs as assessed by the Catwalk gait analysis. Only moderate and severe $\mathrm{SCl}$ animals differed significantly from the other groups (Repeated Measures ANOVA: P<0.05). C-D) Shown are the Rump Height Indices (C) and Foot Stepping Angles (D) as measured in the Single Frame Analysis test. Again, only moderate and severe $\mathrm{SCl}$ animals differed significantly from sham-operated animals (ANOVA: $\mathrm{P} \leq 0.03) . * * *$, and $* * *$ correspond to $\mathrm{P}$-values of $<0.05,<0.01$, and $<0.001$, respectively. 
The rotating rod, $55^{\circ}$ Inclined Ladder Test, and Myogenic Motor Evoked Potentials did not improve sensitivity for $\mathrm{SCl}$, but showed identical on/off patterns for supraspinal locomotor control

The patterns for chronic motor deficits (more than one week post injury) are identical as measured by the rotating rod (with forward locomotion), $55^{\circ}$ Inclined Ladder Test, and Myogenic Motor Evoked Potentials. Again, no significant differences between sham and mild SCl were detected for the rotating rod test and MMEPs (Fig. IV-3A\&C; ANOVA: P $\geq 0.41$ ). The $55^{\circ}$ Inclined Ladder Test did show significant differences between sham-operated and mild $\mathrm{SCl}$ animals up to three week post injury (Fig. IV-3D; Bonferroni: P<0.05). Even at six weeks post injury, the $55^{\circ}$ Inclined Ladder climbing and MMEPs were significantly and fully reduced in both moderate and severe $\mathrm{SCl}$ animals, when compared to sham and mild $\mathrm{SCl}$ animals (Bonferroni: $\mathrm{P}<0.05$ ). Nonetheless, even severe $\mathrm{SCl}$ animals showed constant stepping-like hindpaw movements during ladder climbing test tests, although these movements remained largely dysfunctional. In the rotating rod test, any hindlimb movement soon contributed to improvement in outcome, which was also largely due to stomach dragging/frontlimb compensation.

The backwards locomotion test showed improved sensitivity of SCl-induced locomotor disability

Significant and chronic (measured up to six weeks post injury) decreases in locomotor function on the backwards locomotion test were measured for all SCl severities, which specifically includes mild $\mathrm{SCl}$ (when compared to sham-operated animals; Fig. IV-3B; Bonferroni Post-Hoc: P $\leq 0.01$ ). The ability to perform backwards locomotion was strikingly absent in animals with moderate and severe $\mathrm{SCl}$, as virtually no movement in the hindlimbs was noted in these animals during testing. By far most moderate $\mathrm{SCl}$ animals were unable to make even slight hindlimb movements when attempting to walk backwards, even at six weeks post injury, which was in sharp contrast to their front limb movements. 

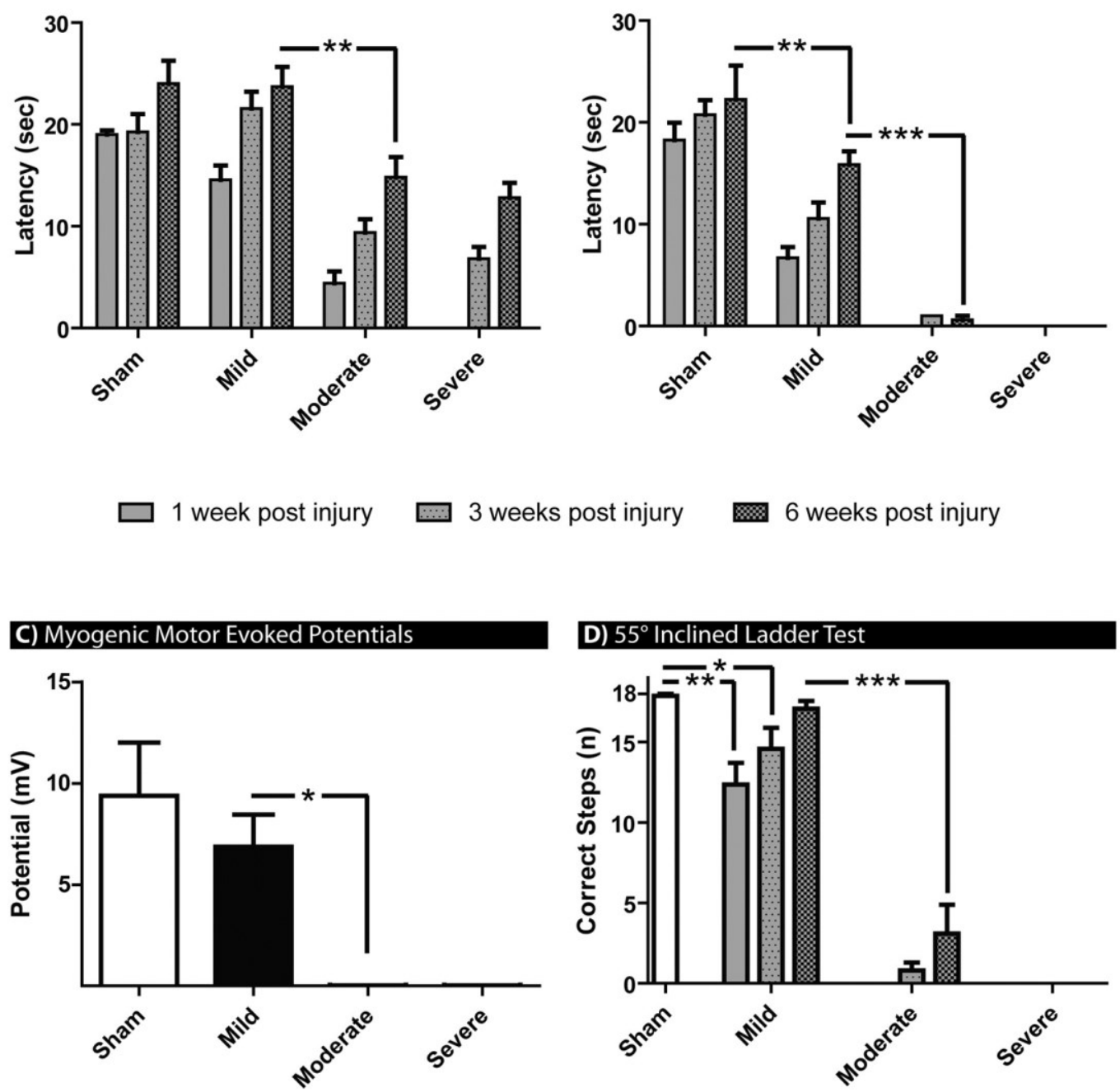

Figure IV-3: Results from MMEP assessments, ladder climbing, and rotating tod tests which depend largely on supraspinal locomotion control. Note the lack of differences between sham-operated and mild $\mathrm{SCl}$ animals in all but the backwards locomotion rotating rod test in the chronic stage post injury. A) Displayed are drop-off latencies of the rotating rod testing with forward locomotion. Only differences between mild and moderate $\mathrm{SCl}$ animals, not sham and mild, or moderate and severe $\mathrm{SCl}$ animals, were significant (Repeated Measures ANOVA), for all three timepoints (Bonferroni Post-Hoc: P<0.01). B) Displayed are dropoff latencies of the backwards locomotion rotating rod test. The backwards locomotion rotating rod test was the only test which showed significant differences between sham-operated, mild, and moderate $\mathrm{SCI}$ animals, at all time points (Bonferroni Post-Hoc: P $\leq 0.01$ ). C) Shown are the Myogenic Motor Evoked Potentials measured in the $\mathrm{m}$. gastrocnemius after transcranial electric stimulation at six weeks post injury. Only between mild and moderate $\mathrm{SCl}$ occurred a significant drop in evoked potentials (One Way ANOVA: $P<0.05)$. D) Shown are the number of correct steps/paw placements while climbing the rungs of a $55^{\circ}$ Inclined Ladder. Differences at six weeks post injury were only significant between mild and moderate $\mathrm{SCl}$ animals (Bonferroni: $\mathrm{P}<0.001) . * * *$, and $* * *$ correspond to $\mathrm{P}$-values of $<0.05,<0.01$, and $<0.001$, respectively, and are only displayed at the latest time point on which two consecutive injury severity groups showed significant differences. 


\section{Discussion}

This study shows that the backwards locomotion test, unlike regular post-SCI hindpaw locomotor tests, is sensitive enough to allow reliable discrimination between both sham-operated vs. mild SCI animals, and mild vs. moderate $\mathrm{SCl}$ animals in the chronic post-SCI phase. Surprisingly, moderate $\mathrm{SCI}$ animals were virtually unable to perform even the slightest backwards hindpaw movement. Hence, backwards locomotion assessment is not only the first test sensitive enough to detect distinct and chronic differences in locomotor ability between sham, mild and moderate $\mathrm{SCl}$ animals, but also provides a wide continuous scale, from sham to (near) severe injury, which is likely to be of great use for translational therapy evaluations. Furthermore, abovementioned properties of the backwards locomotor test can be hypothesized to be due to the high dependency on supraspinally coordinated sensorimotor control as A) final neural networks of forward and backwards quadruped locomotion are believed to be similar (Musienko 2012), and B) among all performed locomotor tests, the backwards locomotion rotating rod test showed best correlation with 1) MMEPs, 2) acute (noncompensated by autonomic circuitry) scores of the BBB-locomotor score, and 3) deficits in sensory perception thresholds observed in the hindpaws of all animals (see the accompanying manuscript).

Below-level perception only becomes important for locomotor tests when they depend on supraspinal motor control, as sensory input first has to pass through the injury epicenter. Hence, sensory disturbances have been less relevant in locomotor tests like the BBB-locomotor score which depend more on autonomic propriospinal locomotor circuitries (Hochman 2012), These circuitries likely make than even numb rats can walk (Hofstetter 2003), This notion is nicely shown by the $55^{\circ}$ Inclined Ladder Test, where mild SCl animals could normally detect and step on the rungs (largely unaffected hindpaw sensory thresholds; see the accompanying manuscript), while moderate $\mathrm{SCl}$ animals could not (altered sensory function), although the latter still showed pronounced (spinal autonomic) hindlimb motor activity during climbing. The latter is in line with reports showing vast rat spinal cord (forward) locomotor autonomy by propriospinal circuits (Rossignol 2011a, Rossignol 2011b, Tillakaratne 2010).

Although the extent of spinal autonomic locomotion in quadrupeds is interesting by itself, its value is believed to be much less in humans (Battistuzzo 2012, Wessels 2010). It then mostly remains an important hurdle for clinically translatable assessments of long tract regeneration in rats. Interestingly, MMEPs are typically reported not to correlate to basic locomotor testing (Loy 2002), but it does display a pattern more related to the backwards locomotion test and studies in human $\mathrm{SCl}$ patients showed high sensitivities of MMEPs for spinal integrity and functional outcome (Clarke 1994, Di Lazzaro 1999, Di Lazzaro 2004). Hence, the backwards locomotion test seems to correlate 
closely with the integrity of long tracts which corresponds to those which are most relevant for function in humans. Further investigation will show which (combinations of) major descending (cortico-, rubro-, raphe-, reticulo-, vestibule-, and coerulo-spinal) tracts rat backwards locomotor ability depends, and if (spontaneous) reconnectivity in any of these tracts corresponds with recovery.

In conclusion, we recommend backwards locomotion testing for better translatability of experimental $\mathrm{SCl}$ research. 


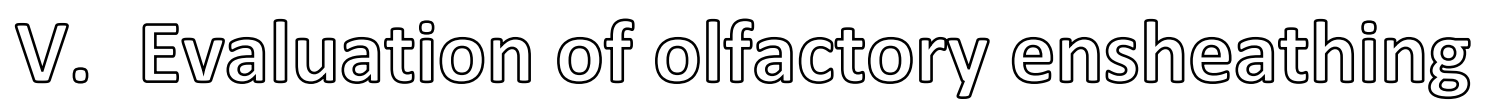

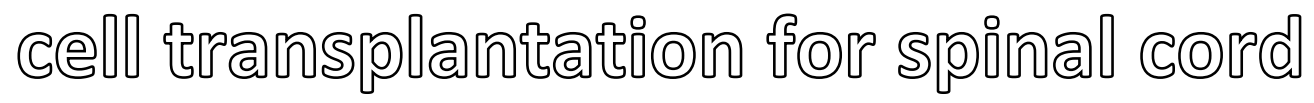

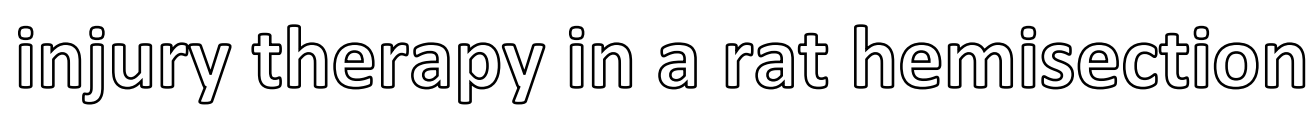 n๐@อ!}




\title{
Motor outcome and allodynia are largely unaffected by novel olfactory ensheathing cell grafts to repair low- thoracic lesion gaps in the adult rat spinal cord
}

\author{
Ronald Deumens; Sebastiaan van Gorp, Ahmed Bozkurt, Christina Beckmann, Tobias Führmann, \\ Katrin Montzka, Rene Tolba; Eiji Kobayashi; Ingo Heschel, Joachim Weis, Gary A Brook
}

Behavioral Brain Research

\begin{abstract}
Olfactory ensheathing cells $(\mathrm{OEC})$ are a promising graftable cell population for improving functional outcomes after experimental spinal cord injury. However only few studies have focused on experimental models with large cavitations, which require bridging substrates to transfer and maintain the donor cells within the lesion site. Here, a state-of-the-art collagen-based multichanneled three dimensional scaffold was used to deliver olfactory ensheathing cells to $2 \mathrm{~mm}$ long unilateral low-thoracic hemisection cavities. For a period of 10 weeks, allodynia of the hindpaws was monitored using the Von Frey hair filament test, while an extensive analysis of motor ability was performed with use of the CatWalk gait analysis system and the BBB locomotor scale. No substantial improvement or deterioration of motor functions was induced and there was no effect on lesioninduced allodynia. On the basis of these data, we conclude that relatively large spinal cord lesions with cavitation may present additional hurdles to the therapeutic effect of OEC. Future studies are needed to address the nature that such lesion cavities place on cell grafts.
\end{abstract}




\section{Introduction}

Spinal cord injury $(\mathrm{SCl})$ has dramatic effects on the individuals concerned as well as on society. Debilitating consequences such as deficits in motor function and development of neuropathic pain are directly related to the disruption of spinal tissue and the cellular and molecular processes induced by trauma (Berger 2011, Hulsebosch 2009). Many different experimental approaches have been used to promote tissue repair or plasticity (e.g. regeneration of severed axon tracts) (Berger 2011). Delivery of axon-growth promoting cells into the injured spinal cord has proven to be a popular approach in experimental medicine, and clinical trials have been initiated. In particular, olfactory ensheathing cells (OEC) have been considered a highly promising population of cells for $\mathrm{SCl}$ repair because they support the regeneration of olfactory nerve fibers from the periphery into areas of the central nervous system (CNS) throughout the life-span of mammals (Raisman 2007).

OEC express many neurotrophic factors, and unlike many other types of cells can be applied as autografts, thereby by-passing side-effects of immunosuppression that would be required for allografts or xenografts (Munoz-Quiles 2009), while requiring minimal pre-grafting in vitro manipulations. A main advantage of grafting OEC into lesioned CNS is their ability to intermingle with- and migrate amongst CNS glia, such as astrocytes (Lakatos 2000). Moreover, OEC tend to form and maintain open channel-like structures that form a regenerative substrate, not only in their normal habitat (Li 2005), but also when transplanted into small electrolytic lesions of the of the corticospinal tract (Li 1998). Implanting OEC into partial or complete lesions of the rat spinal cord has been found to induce autonomic and voluntary motor improvements (Li 2003, Ramon-Cueto 2000).

However, when considering human $\mathrm{SCl}$ with the formation of large cystic cavitation, it is unlikely that grafting donor cells alone will be sufficient to promote repair. The bridging of large cystic cavities requires a physical implant which would act as a carrier for the donor cells and thus be a substrate capable of supporting nerve fiber regeneration. We have previously demonstrated the cytocompatibility of a degradable biomatrix, (based on polylactic acids) with OEC (Deumens 2004). However, implantation of such OEC-polylactide matrices into cavities of the injured rat spinal cord did not lead to clear improvements in functional outcome (Deumens 2006b). A main limitation in the latter approach may have resided in the fact that OEC were cultured on the surface of the matrices before grafting, rather than throughout the matrices' internal 3D micro-architecture.

A major advance in tissue engineering for long distance axon regeneration within the lesioned CNS has been the development of a 3D collagen-based scaffold containing longitudinally orientated micro-channels that demonstrates excellent cytocompatibility with a range of neural cells including dorsal root ganglion neurons, SH-SY5Y neuroblastoma, human neural progenitor-derived astrocytes, 
rat Schwann cells, rat astrocytes, and rat OEC (Bozkurt 2007, Bozkurt 2009, Fuhrmann 2010, Mollers 2009). In the present study, OEC-seeded or non-seeded collagen scaffolds were implanted into unilateral $2 \mathrm{~mm}$ resection thoracic spinal cord injuries of the adult rat. This model of SCl was selected because of its relevance to study both motor deficits and symptoms of neuropathic pain (Bennett 2000a, Christensen 1996). Lesioned animals that received no treatment served as controls. Motor outcome and allodynia were investigated over a post-surgical period of ten weeks. Motor performance was studied using the conventional BBB locomotor test and the CatWalk gait analysis system. Allodynia was assessed using the von Frey hair filament test.

\section{Animals, methods, and materials}

Female adult Lewis rats (Charles River, Germany) weighing 185-220 grams used for the present study were maintained in accordance with the guidelines of the German animal protection statute and experimental protocols were approved by the German governmental ethical committee. Every attempt was made to minimize the animal number as well as any pain or discomfort. Animals were housed under temperature-controlled conditions at $21 \pm 1{ }^{\circ} \mathrm{C}$, with a normal 12:12h light/dark cycle with ad libitum access to water. A diet restriction protocol (15g chow/rat/day) was used during a 2week pre-operative period of CatWalk training (see below) and once per week (the day preceding post-operative CatWalk testing). During the rest of the study, food was available ad libitum.

The two weeks preceding surgery were used for daily training of animals on a $1.5 \mathrm{~m}$ long CatWalk runway (Noldus Information Technology, The Netherlands). This training period resulted in rats making consecutive, uninterrupted runs with crossing times between 1.1 and 1.6 seconds. The methodology of CatWalk training is described extensively elsewhere (Deumens 2006a). During the week before surgery, animals were habituated to the von Frey set-up used to obtain paw withdrawal thresholds to mechanical stimulation. This set-up consisted of Perspex boxes positioned on a wiremesh surface. Animals were allowed to get used to the apparatus for a minimum of 15 minutes before testing. The hindpaw withdrawal threshold (PWT) to mechanical stimulation was assessed using von Frey hair filaments of increasing thickness (0.41, 0.69, 1.20 1.40, 2.00, 3.63, 5.50, 11.70, 15.14, and $28.84 \mathrm{~g}$ ) and the up-down method (Chaplan 1994). Following three pre-operative sessions, baseline PWT values were obtained.

Adult GFP-transgenic Lewis rats were used to obtain OEC as described previously (Alexander 2002). Primary cultures of $\mathrm{OEC}$, reaching near confluence by 10 days in vitro were enriched using magnetic cell sorting (MACS) and the low-affinity nerve growth factor (NGF) p75 receptor for separation. The OEC were seeded onto $2 \mathrm{~mm}$-long cylindrical collagen scaffolds with a diameter of $2 \mathrm{~mm}$ (Matricel $\mathrm{GmbH}$, Herzogenrath, Germany) at a number of 400,000 cells in a volume of $20 \mu \mathrm{L}$. OEC were 
allowed to adhere to the scaffold for $30 \mathrm{~min}$ before adding growth-factor-containing medium. At $24 \mathrm{~h}$ after seeding, the OEC-seeded scaffolds were used for transplantation. Control scaffolds were treated identically but without OEC.

Animals received a subcutaneous injection with Buprenorphine (Temgesic $0.1 \mathrm{mg} / \mathrm{kg}$ body weight; Schering-Plough, Utrecht, The Netherlands) 30-60 min before surgery. Anesthesia was induced by $5 \%$ isoflurane with air as carrier gas at a flow rate of $250 \mathrm{~mL} / \mathrm{min}$ using a U-400 anaesthesia unit (Agntho's, Lidingö, Sweden) with an open mask system. When the corneal blink reflex and paw withdrawal reflexes were absent, maintenance of anesthesia was set at $2 \%$ isoflurane. An ophthalmic ointment was applied to the eyes to prevent drying during surgery. Then, the back of the animal was shaved and disinfected before making a skin incision. A laminectomy of the T10-11 vertebral level was performed, exposing the T13 spinal cord level. The dura was opened gently and four suture stitches ( $9 / 0$ sutures of monofilament polyamide; $9 / 0$ Ethilon ${ }^{\circledR}$, Ethicon Inc., Somerville) were positioned at the corners of the opened dural membrane. A right-sided hemisection, about 2.0 $\mathrm{mm}$ long, was created using a no.10 scalpel blade and microscissors. Care was taken to prevent damage to the major dorsal blood vessels or vascular branches. Completeness of hemisection was verified microscopically following aspiration of the lesion site. Animals were divided into three groups at random: (1) receiving no implant $(n=10),(2)$ receiving an empty collagen scaffold $(n=10)$, and (3) receiving an OEC-seeded collagen scaffold $(n=10)$. Dural sutures were used to stabilize the implant after which muscle and skin were closed with $4 / 0$ single suture stitches (4/0 Prolene ${ }^{\circledR}$, Ethicon Inc., Somerville). Three animals died during surgery due to respiratory depression and four animals showed inappropriate deficits in both hind paws instead of unilateral deficits (no weight support up until four weeks post-surgery) probably due to local bleeding and/or edema. As a consequence, the numbers of animals were 9 control animals without graft, 7 animals receiving empty collagen scaffolds, and 7 animals receiving OEC-seeded collagen scaffold.

During the first week after surgery, BBB scores were obtained at post-operative day (dpo) 1, 3, 5 and 7 , and thereafter on a weekly basis. Although both hindpaws were scored in this test, deficits were exclusively detected in the hind paw ipsilateral to the lesion. Weight support was regarded a prerequisite for reliable testing with the von Frey filaments and this was observed in the hindpaws of all animals from $21 \mathrm{dpo}$. PWT were obtained at four weeks and at 10 weeks post-surgery. Postoperative CatWalk data were acquired at these same time points (before obtaining PWT to mechanical stimuli).

Behavioral data were analysed using the SPSS 15.0 software. A general linear model (GLM) with repeated measures (for time) used to analyze overall time, group, and time $\mathrm{x}$ group interaction 
effects (Dunnett's post-hoc correction with untreated animals as control reference). In case of an overall time difference, an Analysis of Variance (ANOVA) was used to analyze effects at individual time points (Dunnett's post-hoc correction with pre-operative baseline values as control reference). Data are indicated as mean \pm standard error of the mean (SEM). A $p$-value of 0.05 was considered as the level of statistical significance.

\section{Results}

Unilateral hemisection of the low-thoracic spinal cord in rat resulted in onset of hypersensitivity to mechanical stimulation of both hind paws, as reported previously (Christensen 1996). Untreated animals showed a decrease in PWT to mechanical stimulation of the ipsilateral hind paw of about $40 \%$ and $75 \%$ at 4 and 10 weeks after injury, respectively (Fig. V-1A). The decrease in PWT of the contralateral hindpaw was delayed compared to the ipsilateral hind paw (Fig. V-1B). Statistical testing showed an overall time difference in ipsilateral PWT $\left(F_{2,20}=36.6 ; p<0.001\right)$ and contralateral PWT $\left(F_{1,20}=23.5 ; p<0.001\right)$. However, no significant group differences were found, neither for ipsilateral nor for contralateral PWT.

Robust locomotor deficits could be detected using the BBB scale. BBB scores decreased immediately after surgery and a gradual and spontaneous recovery was observed over a period of about two weeks after which a plateau phase was reached. Scores dropped to around 8.5 at five days and around 14.5 at four and ten weeks after injury. BBB scores did not differ for the three different animal groups. At the end point of the investigation (10 weeks after SCI), BBB scores were $13.7 \pm 0.6$, $15.3 \pm 0.8$, and $14.5 \pm 0.7$ for untreated animals, scaffold-treated animals, and OEC-scaffold-treated animals, respectively. Post-mortem analysis confirmed the reproducibility of the lesion in this investigation with no statistically significant differences in lesion size for the three different animal groups (data not shown).

In order to objectively determine more delicate changes in motor function due to the spinal cord lesion with- or without treatment, CatWalk gait analysis was performed. Importantly, the crossing times required for the CatWalk runs did not differ for time or treatment. This fact is of vital importance to the interpretation of the data, because speed of gait has a strong impact on most gait parameters. We found that unilateral low-thoracic spinal cord injury in the rat affects some, but not all parameters recorded by gait analysis. The stride length (i.e. the distance between two subsequent placements of a single paw) was for example unaffected for both hind paws (data not shown). Also, the regularity index, which is a measure of coordination, remained relatively stable after injury (Fig. V-1C). The most prominent motor deficits detected by the CatWalk gait analysis turned out to be the base-of-support (BOS) of the hind paws (i.e. the distance between both hind 
paws) (Fig. V-1D). Indeed, the animals tended to place their ipsilateral hind paw much more laterally after $\mathrm{SCl}$. This was reflected by an increase in the BOS from about $1.5 \mathrm{~cm}$ at pre-injury baseline to about $3.0 \mathrm{~cm}$ after injury (Fig. V-1D). Statistical analysis showed a strong time effect $\left(F_{2,20}=82.7\right.$; $p<0.001)$, but no group effect. Also the ratio of the print area of the ipsilateral over the contralateral hind paw was significantly reduced following SCl (Time effect: $F_{2,20}=45.8 ; p<0.001$ ) (Fig. V-1E), but without treatment effects. The ratio of paw print intensity (ipsi/contra) was slightly reduced by the $\mathrm{SCl}$ (Fig. V-1F). However, this decrease did not always reach statistical significance. No group/treatment differences were found for this measure either.

A

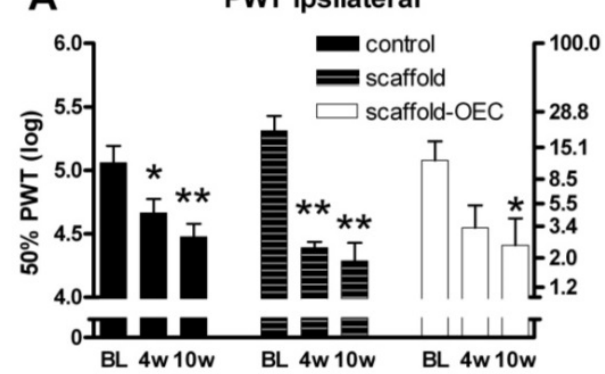

C
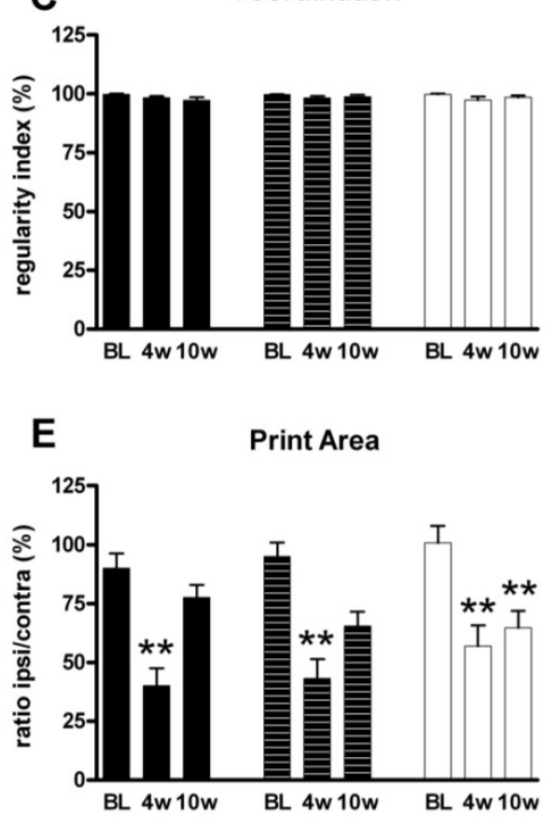

B

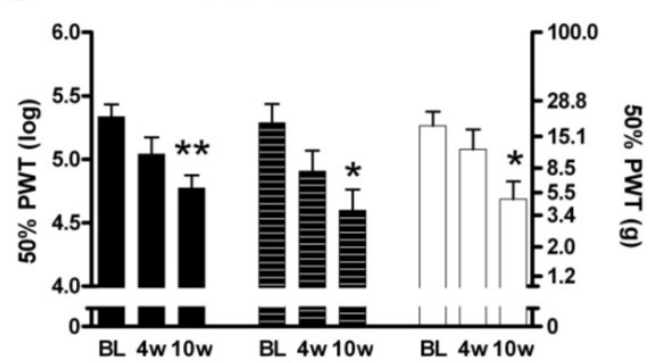

D

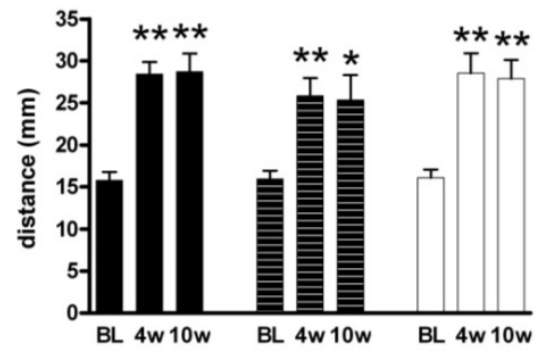

$\mathbf{F}$

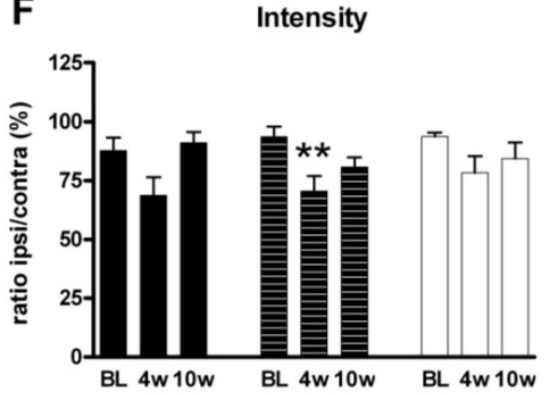

Figure V-1: Effects of OEC-collagen repair strategy of unilateral low-thoracic spinal cord hemisection. A,B: the 50\%-paw withdrawal threshold (PWT) is shown at baseline (BL), 4 weeks and 10 weeks after injury for the ipsilateral (A) as well as contralateral (B) hind paw of animals without treatment (open histograms), treated with empty scaffolds (striped histograms), and treated with OEC-scaffolds (dotted histograms). C-F: CatWalk parameters are shown for the different animal groups at baseline (BL), 4 weeks and 10 weeks after injury; note that the regularity index is calculated taking all paw into consideration while the base-ofsupport, print area and intensity are calculated in relation to the hind paws (ratio ipsi/contra for print area and intensity). 


\section{Discussion}

Transplanted OEC have, for some time, been regarded as having substantial therapeutic potential in $\mathrm{SCl}$ and have even been subject to clinical trials (Mackay-Sim 2008). Implanting OEC seems to be of major therapeutic benefits including their supportive effect on the regeneration of many different descending fiber systems (Deumens 2005) and their re-myelination potential. Earlier studies by the group of Raisman had shown that injections of OEC into highly defined, small, electrolytic lesions of the rat corticospinal tract induced substantial recovery of motor function, possibly due to the regeneration of severed corticospinal fibers ( $\mathrm{Li}$ 1998). The functional benefits of implanting suspensions of OEC have not only been limited to small spinal cord lesions. When implanted into complete thoracic spinal cord transection injuries, donor OEC were found to enhanced weight support and even locomotor function (Ramon-Cueto 2000). Although the completeness of the spinal lesion increases the challenge to spinal cord repair, it does not necessarily represent a good working model for cystic cavitation, which is an important aspect of human $\mathrm{SCl}$. Large spinal cord cavitations require bridging strategies in which donor cell (e.g. OEC) transfer needs an implantable biomaterial and preferably present an orientated substrate capable of promoting efficient and directional axon regeneration. Raisman and co-workers reported on a method of adjusting implanted OEC to include their own dense extracellular matrix, but did not test this method in a model of larger cavition (Li 2003). An alternative strategy adopted by other research groups has been to use bioengineered scaffolds, from synthetic or biological polymers, to act as cell carriers and guidance frameworks for axon regeneration across $\mathrm{SCl}$ lesion cavities. It is still very early for clear statements on the therapeutic potential of such approaches and much more research in this area is needed. We previously found that immediate or delayed implantation of OEC on polylactide-based matrices into thoracic dorsal hemisection cavities did not improve motor function, nor were any regenerative effects of substance observed (Deumens 2006b). An obvious factor influencing the therapeutic effect of this type of tissue engineering intervention strategy is the choice of the material. Polylactic acid may be sub-optimal as it may acidify the implant environment once it breaks down. In recent years, we have focused our attention on the use of a novel, state-of-the-art collagen-based biomaterial, which is highly porous and contains multiple longitudinally orientated microchannels. In vitro studies have indicated that this three-dimensional scaffold can be seeded with both neuronal and non-neuronal cells, which readily adhere to and grow within the scaffold (Bozkurt 2007, Bozkurt 2009, Fuhrmann 2010, Mollers 2009). Importantly, OEC were found to migrate and proliferate within the scaffold (Mollers 2009). In the present investigation, we implanted OEC-collagen scaffolds in the cavities created following unilaterally hemisectioning of the low-thoracic spinal cord of adult rats. A major advantage of donor OEC over many other types of graftable cells is their ability to interact 
with host astrocytes. Indeed, other cell types, including Schwann cells, form physical as well as molecular boundaries when confronted with astrocytes (Lakatos 2000).

The main aim of our investigation was to study the effect of a tissue engineering strategy on motor and sensory parameters after experimental $\mathrm{SCl}$. Motor deficits and allodynia present two major symptoms which negatively affect the quality of life of an individual living with $\mathrm{SCl}$. Experimental work has shown that treatment approaches which affect plasticity in neural systems may have opposing consequences on pain and motor function (Deumens 2008, Hofstetter 2005). Increased plasticity in severed fiber tracts as well as local spinal cord neuronal circuitries is generally though to benefit motor functions. Increased plasticity in nociceptive circuits of the spinal cord on the other hand might underlie neuropathic pain (Berger 2011). As such it is of utmost importance to consider both of these two outcomes in preclinical investigations. The use of the CatWalk gait analysis system in the present study allowed a detailed investigation of gait parameters during locomotion of the lesioned animal. Most strikingly, the base-of-support of the hind limbs was increased 2-fold in this injury model. Implantation of OEC-seeded or empty collagen scaffolds into the lesion cavity exerted no demonstrable effect on BOS or any other motor or sensory parameters of the present investigation. Our findings may reflect (1) the difficulty in obtaining functional benefits by approaches to repair cavities in the injured spinal cord, (2) that the concentration of donor cells may not have been sufficient for such a lesions, (3) that trophic support has to be added to the grafting procedure, and/or (4) that some degree of distortion of the scaffold may have occurred or that the chosen lesion model may simply not have been the best for demonstrating implant-induced effects. Despite these rather disappointing preliminary results, we are still hopeful for the future. It seems essential to determine what limits the therapeutic effects of OEC grafting in spinal cord lesion cavities. Obviously, inflammatory mediators may restrict grafted OEC within the inflammatory loci (Su 2009), which may an OEC-supported long-distance regeneration of severed fibers. Concomitant immune modulatory approaches might then be required to optimize the therapeutic effects of this type of cell grafting therapy. Interestingly, immune modulatory therapies such as administration of the TNF-decoy receptor etanercept, effectively reduces allodynia after experimental SCI (Marchand 2009).

In conclusion, the present investigation showed that implantation of a collagen-based scaffold, seeded with OEC or unseeded, did neither improve nor deteriorate motor outcome and allodynia following thoracic spinal cord hemisection in the rat. The most reasonable interpretation of such data is that this particular combination of donor cells and scaffold is not the best for this particular lesion model associated with clinically relevant lesion cavities. As cavitation is closely related to 
human $\mathrm{SCl}$, we feel that experimental repair approaches need to ultimately be tested for their efficacy in models containing cavities. Lack of therapeutic effect, then needs to be investigated more thoroughly to improve repair approaches, thereby getting closer to clinical treatments of the severe condition $\mathrm{SCl}$ represents. 


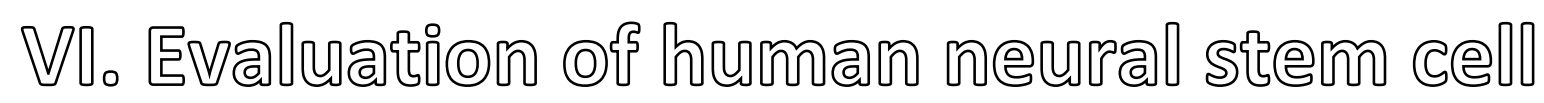

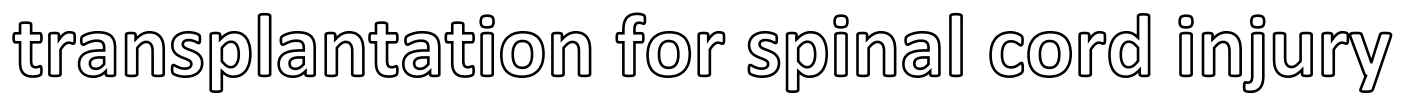

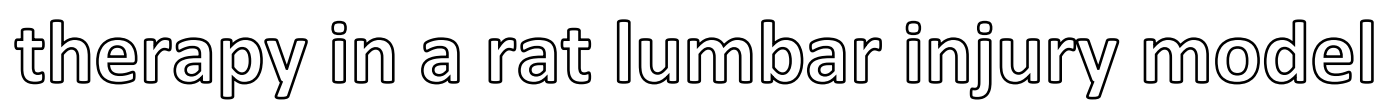




\title{
Amelioration of motor/sensory dysfunction and spasticity in a rat model of acute lumbar spinal cord injury by human neural stem cell transplantation.
}

Sebastiaan van Gorp; Marjolein Leerink, Osama Kakinohana; Olexandr Platoshyn; Camila Santucci; Jan Galik;

Elbert A. Joosten; Marian Hruskan-Plochan; Danielle Goldberg; Silvia Marsala; Karl Johe; Martin Marsala

\author{
Stem Cell Research \& Therapy
}

\begin{abstract}
Intraspinal grafting of human neural stem cells represents a promising approach to promote recovery of function after spinal trauma. Such a treatment may serve to: 1) provide trophic support to improve survival of host neurons, 2) improve structural integrity of spinal parenchyma by reducing syringomyelia and scarring in trauma-injured regions, and 3) provide neuronal populations to potentially form relays with host axons, segmental interneurons, and/or $\alpha$-motoneurons. Here we characterized the effect of intraspinal grafting of clinical grade human fetal spinal cord-derived neural stem cells (HSSC) on recovery of neurological function in a rat model of acute lumbar (L3) compression injury. Three-month-old female Sprague-Dawley rats received L3 spinal compression injury. Three days post-injury, animals were randomized and received intraspinal injections of either HSSC, media-only, or no injections. All animals were immunosuppressed with tacrolimus, mycophenolate mofetil, and methylprednisolone acetate from the day of cell grafting and survived for 8 weeks. Motor and sensory dysfunction was periodically assessed using open field locomotion scoring, thermal/tactile pain/escape thresholds and myogenic motor evoked potentials. The presence of spasticity was measured by gastrocnemius muscle EMG response during computercontrolled ankle rotation. At end-point, gait (Catwalk), ladder climbing, and single frame analyses were also assessed. Syrinx size, spinal cord dimensions, and extent of scarring were measured by MRI. Differentiation and integration of grafted cells in the host tissue were validated with immunofluorescence staining using human-specific antibodies. Intraspinal grafting of HSSC led to a progressive and significant improvement in lower extremity paw placement, amelioration of spasticity, and normalization in thermal and tactile pain/escape thresholds at 8 weeks post-grafting. MRI volume reconstruction and immunofluorescence analysis of grafted cell survival showed near complete injury-cavity-filling by grafted cells and development of putative GABA-ergic synapses between grafted and host neurons. Peri-acute intraspinal grafting of HSSC can represent an effective therapy which ameliorates motor and sensory deficits after traumatic spinal cord injury.
\end{abstract}




\section{Introduction}

Extensive experimental and clinical data show that the mechanisms leading to a clinically-defined loss of neurological function after spinal trauma can in general be considered in two categories. First is the pathology and corresponding functional loss resulting from a direct mechanical injury of axons at the injury epicenter, and second is a progressive appearance of secondary changes (local edema, hematoma, excitotoxicity and ischemia) which can evolve over hours to weeks after the initial impact (for review see Hagg \& Oudega (Hagg 2006)). Consistent with our current knowledge of the mechanism which leads to the development of secondary post-injury cascade, the current experimental and clinical treatment strategies primarily focus on: 1) improvement of local metabolism and blood flow (e.g., through decompression therapy and hypothermia) (Jones 2012, Oudega 2012), and 2) modulation of local inflammatory response (e.g., with methylprednisolone) (Ankeny 2009, Bracken 2012, David 2011, Fleming 2006).

A separate group of experimental treatment modalities are aimed at improving the local neurotrophic activity at and around the injury epicenter with the primary goal of increasing the survival of partially injured axons and/or neurons. In this category of experimentation, besides the use of locally delivered trophic factors (such as BDNF-, GDNF-, and FGF-peptides or growth factorsgene-encoding vectors) (Lu 2008), regionally grafted fetal or embryonic stem cell-derived neuronal precursors are frequently used (Llado 2004, Sahni 2010). In addition, the use of neuronal precursors for spinal grafting and development of functional synapses between grafted neurons and the neurons of the host can serve to create a functional "relay" system throughout the injured spinal cord region. In these studies, for example, it has been demonstrated that acute grafting of fetal rat spinal cord-derived neuronal and glial-restricted precursors into a site of cervical dorsal column lesion leads to formation of functional ascending spinal relays in vivo as validated electrophysiologically and histologically in the dorsal column nucleus (Bonner 2011). Similarly, using electrophysiologically-defined phrenic nerve activity and qualitative histology, functional restoration of the spinal phrenic nucleus circuitry was demonstrated after acute grafting of fetal rat spinal cordderived neural precursors into the injury epicenter in rats (White 2010). Embryonic rat cerebral cortex-derived neural precursors, grafted acutely or at 4 weeks post-injury into the epicenter of a spinal kainic acid-induced lesion, showed good engraftment up to 12 weeks after grafting (Magnuson 2001). Consistent with histologically and electrophysiologically validated maturation and functional incorporation of grafted neuronal cells in previously trauma-injured or chemicallylesioned spinal cord, a beneficial behavioral effect was also noted in several experimental studies. Acute spinal grafting of mouse neural precursors (isolated from fetal neural tube tissue) into the epicenter of L2 spinal cord compression injury (10 g for $5 \mathrm{~min}$ ), improved hindlimb motor and 
sensory function in mice (Boido 2011). Similarly, acutely grafted human embryonic stem cell-derived oligodendrocyte progenitors and/or motoneuron progenitors into a complete thoracic transection model resulted in partial restoration of hindlimb locomotor function and motor evoked potentials in rats (Erceg 2010). Delayed (7 days post injury) grafting of neurally pre-differentiated mouse embryonic stem cells into quisqualic acid-lesioned lumbar spinal cord led to a reduction in spontaneous and thermally-, mechanically-, or chemically (paw formalin injection)-evoked pain-like behavior in mice (Hendricks 2006).

Recently, well-defined protocols were developed which permit the isolation and long-term stable expansion of (non-immortalized) human fetal brain or spinal cord tissue-derived neural stem cells (Carpenter 1999, Ferrari 2010, Svendsen 1998, Uchida 2000, Vescovi 1999, Yan 2006). Using these protocols, continuing neurogenic potential, as evidenced by neuronal differentiation and the ability of differentiated neurons to generate action potentials in vitro, was documented at even high $(>20)$ passage numbers (Guo 2010, Vescovi 1999). Some of these lines were successfully used for: 1) generation of GMP-grade clonally-derived cell lines, 2) extensive pre-clinical evaluation using a variety of neurodegenerative small and large animal models, and 3) subsequently used successfully in Phase I human clinical trials (Jacobs June 2011, Neuralstem Inc. - Emory University 2000, StemCells Inc. 2000 - cited: 2012 Aug 10).

In our previous studies, we have extensively characterized the in vivo treatment effect after spinal grafting of cGMP-grade human fetal spinal cord-derived stem cells (NSI-566RSCs line) using a spinal ischemia model in rats and transgenic rat model of ALS $\left(S O D 1^{G 93 A}\right)$. In those studies, we have shown that: 1) grafting of NSI-566RSCs into lumbar spinal cord of adult Sprague-Dawley (SD) rats with previous spinal ischemic injury is associated with a progressive improvement of ambulatory function which correlates with long-term grafted cell survival and extensive neuronal differentiation (Cizkova 2007), and 2) bilateral lumbar grafting of NSI-566RSCs in pre-symptomatic SOD ${ }^{693 A}$ rats provides a transient functional benefit and suppression of $\alpha$-motoneuron degeneration, i.e., a protective effect which was absent in media-injected animals (Hefferan 2012). Using the same cell line, we have also demonstrated the optimal dosing regimen and safety after grafting into the lumbar spinal cord of immunosuppressed minipigs (Cizkova 2007). The dosing design defined in this pre-clinical minipig study was then subsequently used in a recently completed Phase I human clinical trial in ALS patients receiving lumbar and cervical grafts of NSI-566RSCs (Boulis 2011, Neuralstem Inc. - Emory University 2000). In a more recent study using an immunodeficient rat model of complete spinal cord Th3 transection, it was shown that NSI-566RSCs or rat embryonic neural precursor cells, embedded in a fibrin matrix with trophic factors and grafted one week after injury, were able to form functional 
relays. The formation of functional relays was validated behaviorally (BBB locomotor score), electrophysiologically (spinal cord evoked potentials), and histologically (host on graft and graft on host synapses) (Lu 2012).

The goal of our present study was to characterize the effect of NSI-566RSCs grafted spinally in a clinically relevant L3 spinal compression model in continuously immunosuppressed adult SD rats. The presence of treatment effect was assessed by analysis of 1) motor and sensory function, 2) myogenic motor evoked potentials (MEPs), 3) spasticity response during computer-controlled ankle rotation, and iv) qualitative analysis of grafted cell survival and maturation.

\section{Material and Methods}

\section{Animals and surgeries}

All animal studies were approved by the University of California, San Diego Institutional Animal Care and Use Committee. The study design is outlined in Fig. 1. Twelve-week-old Female Sprague-Dawley rats were anesthetized with isoflurane (5\% induction, $1.5-2 \%$ maintenance; in room air) and placed into a Lab Standard Stereotaxic frame (Stoelting, Cat\# 51600, Wood Dale, IL, USA). The animal was elevated $2 \mathrm{~cm}$ by placing it on a homeothermic heating blanket (set at $37^{\circ} \mathrm{C}$ with feedback from the rectal thermometer, Harvard Apparatus, Cat\# 507214, Holliston, MA, USA) which sits on a plastic rectangular block. The animal was then placed in Spine Adaptors (Stoelting, Cat\# 51695, Wood Dale, IL, USA) and a wide Th13 laminectomy was performed using an air-powered dental drill and binocular microscope (exposing the dorsal surface of spinal segment L3). An acrylic rod ( $\varnothing 2.9 \mathrm{~mm}$, length $15 \mathrm{~cm} ; 35 \mathrm{~g}$ ) was then slowly lowered on the exposed L3 segment until it slightly touched the spinal cord but without inducing any compression. The laminectomy site was then filled with mineral oil in which the tip of a small thermocouple (Physitemp, Cat\# IT-14, Clifton, NJ, USA) was submerged and touched the dura. The light from the two fiber optic light pipes of the surgical light (Fiber-Lite, Cat\# MI-150 \& BGG1823M, Dolan-Jenner, Boxborough, MA, USA) were focused on the surgical site (and directly illuminating the temperature probe). Next, the light intensity was manually regulated so that the spinal cord $/$ mineral oil was warmed to $37^{\circ} \mathrm{C}$ and remained at $37 \pm 0.3^{\circ} \mathrm{C}$. If necessary, a $100 \mathrm{~W}$ infrared lamp was used to gradually adjust and maintain the animal's core temperature at $37^{\circ} \mathrm{C}$ (rectal). When both temperatures (i.e., paraspinal and rectal) were at $37 \pm 0.3^{\circ} \mathrm{C}$ for at least 5 min, the rod was slowly lowered until its weight fully rested, perpendicularly, onto the spinal cord. The rod was kept in place for $15 \mathrm{~min}$, while both temperatures were maintained at $37 \pm 0.3^{\circ} \mathrm{C}$. After spinal compression, the rod and mineral oil was removed and the wound sutured in anatomical layers. 


\section{Post-surgical care}

Buprenorphine (0.05 mg/kg, s.c., Reckitt Benckiser, Richmond, VA, USA), $5 \mathrm{~mL}$ of lactated ringer's, 10 $\mathrm{mg} / \mathrm{kg}$ of Cefazolin (Novaplus/Sandoz, Holzkirchen, Germany), and standard triple antibiotic ointment to cover the incision site (Bacitracin, Neomycin, Polymyxin B) was given after every surgery. Bladders were manually emptied twice daily (if full). Sulfamethoxazole and Trimethoprim USP oral suspension (200 mg \& 40 mg per $250 \mathrm{~mL}$ drinking water, Hi-Tech Pharmacal, Amityville, NY, USA) was given for at least 10-14 days after $\mathrm{SCl}$ or until autonomic bladder voiding occurred and for 1-2 days after any other surgery (sham or grafting). Food was provided by placing it at the bottom of cage and water bottles with an elongated drinking tube were used, until regular overhead supplies could be reached by the animal. Animals diagnosed with bacterial infections throughout the study were treated with Sulfamethoxazole (as above), $10 \mathrm{mg} / \mathrm{kg} / \mathrm{day}$ of Cefazolin, and lactated ringer's 5 $\mathrm{mL} / 0.5$ day.

\section{Cell derivation and preparation}

The cells, named "NSI-566RSC", were produced by Neuralstem Inc. (Rockville, MD, USA), as described before (Hefferan 2011). Briefly, human spinal cord neural precursors (HSSC) were prepared from the cervical-upper thoracic region obtained from a single eight week fetus. Meninges and dorsal root ganglia were removed and dissociated into a single cell suspension by mechanical trituration in serum-free, modified N2 media (human plasma apo-transferrin, recombinant human insulin, glucose, progesterone, putrescine, and sodium selenite in DMEM/F12). For growth of the HSSC, $10 \mathrm{ng} / \mathrm{ml}$ basic fibroblast growth factor (bFGF) was added to the modified N2 media and expanded serially as a monolayer culture on poly-D-lysine and fibronectin (Johe 1996). Approximately $6.1 \times 10^{\wedge} 6$ total cells were obtained upon the initial dissociation of the spinal cord tissue. The growth medium was changed every other day. The first passage was conducted 16 days after plating. At this point, the culture was composed mostly of post-mitotic neurons and mitotic HSSC. Mainly the mitotic cells were harvested through brief treatment with trypsin and subsequent use of soybean trypsin inhibitor. The cells were harvested at approximately $75 \%$ confluence, which occurred every 5-6 days (20 passages). At various passages, the cells were frozen in the growth medium plus $10 \%$ dimethyl sulfoxide at $5-10 \times 10^{\wedge} 6-10$ cells $/ \mathrm{ml}$. The frozen cells were stored in liquid nitrogen. Upon thawing, the overall viability and recovery was typically $80-95 \%$. A cell bank of passage 16 cells was prepared and used for this study.

For the production of GFP-labeled NSI-566RSC, a Lentiviral vector was constructed containing the human Ubiquitin $C$ promoter driving expression of enhanced green fluorescent protein. Viral particles produced by infected 293FT cells were collected after overnight incubation, then 
concentrated by centrifugation and stored frozen. Neural stem cell cultures were infected by overnight incubation in growth medium supplemented with viral supernatant. Infected stem cells were washed with PBS and cultured as described above. After multiple passages, $>90 \%$ of cells were EGFP positive (assessed after immunohistochemical staining). A cell bank of passage 17 cells was prepared and used for this study.

One day prior to each grafting day, one cryopreserved vial of the previously prepared cells was thawed, washed, concentrated in hibernation buffer, and shipped from the cell preparation site (Neuralstem, Inc., Rockville, MD, USA) to the surgery site (University of California, San Diego, CA, USA) at $2-8^{\circ} \mathrm{C}$ by overnight delivery. Upon receipt the following day, the cells were used directly for implantation without further manipulation. Before and after implantation, the viability of cells was measured with trypan blue (0.4\%; Sigma). Typically, a $>85 \%$ viability rate was recorded.

\section{Inclusion and exclusion criteria, randomization and blinding}

Three days following $\mathrm{SCl}$ and prior to grafting, animals were randomly divided into three groups: the vehicle-injected group, non-injected group, or the HSSC-injected group. $\mathrm{SCl}$ animals with an openfield locomotion score of $\leq 1$ and appearing healthy enough were included. Animals found moribund or automutilating at any point during the study were excluded and euthanized. A total of 42 animals were employed and divided into 6 experimental groups, as follows:

Group A ( $n=14)$ : SCl animals-NSI-566RSC-grafted,

Group B ( $n=10)$ : SCl animals-vehicle-injected,

Group C ( $n=8)$ : $\mathrm{SCl}$ animals-non-injected,

GroupD ( $n=6)$ : sham operated (laminectomy only),

Group E ( $n=6)$ : naïve animals (no surgical manipulation)

Group F ( $n=2)$ : SCl athymic animals-ubiquitin.eGFP ${ }^{+}$NSI-566RSCs-grafted.

One animal was excluded in Group A because of automutilation of the hind paw; two animals were excluded in Group C, one because of automutilation of the hind paw and 1 because of bacterial infection. Six animals had been replaced before dosing/randomization, 5 due to inadequate injuries and 1 because of bacterial infection. 


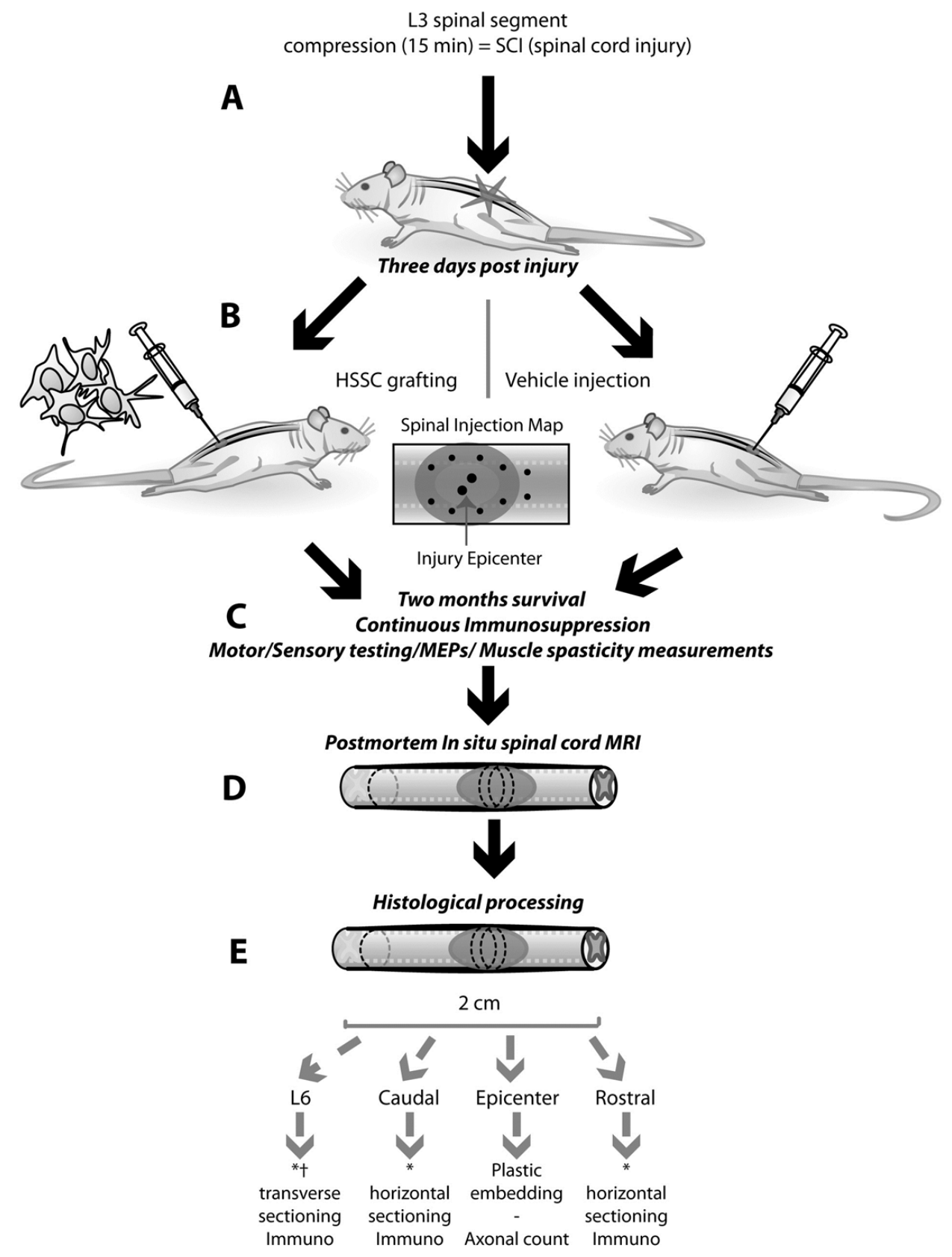

Figure VI-1: Schematic diagram of experimental design. A: To induce spinal cord injury, a $35 \mathrm{~g}$ circular rod was placed on the exposed L3 spinal segment and spinal cord compressed in dorso-ventral direction for 15 min. B: Three days after injury, animals were randomly assigned to experimental groups and received spinal graft of HSSC or media only. A total of 12 injections were performed targeting the injury epicenter and adjacent areas (Spinal Injection Map). C: After spinal injections, animals survived for 2 months while being continuously immunosuppressed and periodically tested for recovery of motor/sensory functions, changes in motor evoked potentials (MEPs) and gastrocnemius muscle spasticity response evoked by computercontrolled ankle rotation. D: At 2 months after treatment, animals were perfusion fixed with $4 \%$ paraformaldehyde and spinal cord MRI-imaged in situ before histological processing. E: After MRI imaging, spinal cords were dissected from the spinal column and spinal blocks prepared for plastic embedding (injury epicenter region) or cryostat sectioning and used for immunofluorescence staining (the regions just above and below the injury epicenter). 


\section{Grafting procedure}

For the intraparenchymal injections, the animals were placed in the stereotactic frame as described above. The L3 spinal cord (i.e., the dura mater) was then re-exposed at the previous laminectomy site. Injections were performed using a 33 gauge beveled needle and $100 \mu \mathrm{L}$ Nanofil syringe (World Precision Instruments, Cat\# NF33BV \& Nanofil-100, Sarasota, FL, USA) connected to a microinjection unit (Kopf Instruments, Cat\# 5000 \& 5001, Tujunga, CA, USA). The duration of each injection was $\geq 45$ sec followed by a $\geq 30 \mathrm{sec}$ pause before slow needle withdrawal. The center of the injection was targeted intermediate of the ventral and dorsal horn and close to the lateral funiculus (distance from the dorsal surface of the spinal cord at $L 3$ level: $0.80 \mathrm{~mm})$. Twelve injections $(20,000$ cells $/ \mu \mathrm{L})$ were done; four injections (0.5 $\mu \mathrm{L}$ each, $0.8-1.0 \mathrm{~mm}$ apart, rostrocaudally) at each lateral boundary of the injury (8 in total), plus two (bilateral) injections ( $0.5 \mu \mathrm{L}$ each) $1.5 \mathrm{~mm}$ caudal from the previous, most caudal injections, and two injections at the core of the epicenter $(1 \mu \mathrm{L}$ at each side of the dorsal vein, bilaterally; see diagram in Fig. VI-1). After the injections, the incision was cleaned with penicillinstreptomycin solution and sutured in two layers.

\section{Immunosuppression}

Two days after injury (i.e., 1 day before grafting), a methylprednisolone acetate (Depo-Medrol, 10 $\mathrm{mg} / \mathrm{kg}$, i.m.) was given, which was repeated thereafter 3 times with $1 \mathrm{mg} / \mathrm{kg} /$ week i.m. Starting directly after grafting, all animals received $3 \mathrm{mg} / \mathrm{kg} / \mathrm{BID}$ s.c. of Tacrolimus (Prograf/FK506, Astellas, Deerfield, IL, USA) until the end of the study. For post-transplant days 0-10, the animals also received $30 \mathrm{mg} / \mathrm{kg} /$ day s.c. of Mycophenolate mofetil (CellCept, Genentech, CA, USA). Immunosuppression was also given to the non-grafted Sprague-Dawley animals (i.e., the naïve, sham operated, and all SCl-control animals).

\section{Open field locomotion testing}

Locomotion recovery after spinal cord contusion injury was monitored using a modified Basso, Beattie, Bresnahan (BBB) open field locomotion rating scale (Basso 1995). The BBB score was modified to reflect the distinct locomotor recovery stages observed after $\mathrm{L} 3 \mathrm{SCl}$. The modified score entailed 8 well-defined degrees of locomotor recovery: 0-1: are identical to the BBB-score, 2: is cumulative score of 2 and 3 of the BBB score, 3: is cumulative score of 4,5 and 6 of the BBB score, 4: is cumulative score of 7 and 8 of the BBB score, 5: reflects weight support with poor paw clearance, 6: is broadened and/or shortened stepping, and 7: is normal walking. In the present study, the locomotor score was obtained before grafting and weekly after injury until the end of the study (i.e., 8.5 weeks post-injury). In addition to a modified BBB score, a regular full 21 scale BBB score was periodically assessed. 


\section{Gait analysis}

The CatWalk apparatus (CatWalk 7.1, Noldus Technology, The Netherlands) was used to quantify gait parameters during walkway crossings (e.g., paw positioning, base of support, stride length, front limb vs. hind limb coordination) by footprint analysis (Deumens 2006b). Animals had to walk down a horizontal glass walkway $(109 \times 15 \times 0.6 \mathrm{~cm}, \mathrm{~L} \times \mathrm{W} \times \mathrm{H})$, of which the glass is illuminated along the long edge. At the end of the walkway, animals had access to their home cage and were given a treat upon arrival (Certified Supreme Mini-Treats ${ }^{\mathrm{TM}}$, Cat\# F05472-1, Frenchtown, NJ, USA). The light only enters the (side of the) glass and reflects merely internally (when the glass is bordered by air). As an animal walks on the glass walkway, light reflects off of the animal's paws, producing a series of bright footprints when viewed through the glass, from below the walkway. The illuminated footprints were then recorded by a video camera with a wide-angle objective that was located underneath the elevated glass walkway. In order to get an optimal contrast between the paws and the surroundings, the test was performed in a room that was totally darkened. The animals were trained for smooth walkway crossing on the 5 days prior to the video acquisitions. To obtain accurate and meaningful data, the following criteria concerning walkway crossings needed to be met: (1) the animal needed to walk uninterrupted across the walkway, at a constant pace, and (2) a minimum of three such crossings per animal were required. Animals without bilateral paw clearance could not be analyzed ( $n=4$ control-SCl animals, and 3 HSSC-treated animals). Digital data analysis consisted of assigning labels (left-fore, left-hind, right-fore, or right-hind) to the animal's paw prints in a recorded walkway crossing, using dedicated CatWalk software. Next, the software calculated gait parameters. Data from the three proper crossings was averaged for statistical analysis.

\section{Inclined ladder test}

The inclined ladder test was performed as described before (Apostolova 2006, Semler 2011). Using an inclined ladder $\left(55^{\circ}\right)$ with twenty $120 \mathrm{~mm}$ wide rungs (diameter: $\left.1 / 4 "\right)$, spaced at equal intervals (60 mm), and having $150 \mathrm{~mm}$-high side walls was used. The rats were trained for this test so that smooth runs were recorded. At the end of the ladder, the animals had access to their home cage and received a treat (as above). The rats were placed at the bottom, and in front, of the ladder. The bottom of the ladder was placed on a $20 \mathrm{~cm}$ elevated platform. Climbing was video recorded from a position below the ladder, so that the ventral aspect of the animal is recorded. All animals were able to climb up the ladder. The correct placing of a hind paw and sustained position until its next forward move was counted over 18 rungs (placement on first and last rung not counted). 


\section{Single frame hind limb motion analysis}

Two parameters were measured in bilateral video captures of animals crossing a runway: the footstepping angle (FSA) and the rump-height index (RHI), as described before (Apostolova 2006, Semler 2011). The FSA is the angle at which the hind paw is placed on the ground just after the swing phase. The angle is defined by a line parallel to the dorsal surface of the paw and a horizontal line behind the paw. Four to six measurements were made for each hind limb (a total of 8 to 12 step cycles). The RHI was defined as the highest point of the base of the tail during the (recorded part of the) run. The values for the left and right paw of each animal were averaged. The elevated runway bar was made of a wooden plate/beam $(1500 \times 150 \times 20 \mathrm{~mm}, \mathrm{~L} \times \mathrm{W} \times \mathrm{H})$. The animals were trained to smoothly walk the beam. Once more, at the end of the beam the animals had access to their home cage and received a treat (as above). The videos (i.e., the selected frames) were selected and analyzed using the video tool VirtualDub 1.9.11 (Written by Avery Lee, http://www.virtualdub.org) and the onscreen measurement tool Screen Ruler V1.0.1a (http://www.caveworks.net).

\section{Myogenic motor evoked potentials}

Animals were anesthetized with ketamine $(80 \mathrm{mg} / \mathrm{kg}$ i.p., Ketaset, Fort Dodge Animal Health, Overland Park, KS, USA). Myogenic Motor Evoked Potentials (MEPs) were elicited by transcranial electrical stimulation (with a pulse duration of $1 \mathrm{~ms}$ at $7 \mathrm{~mA}$ using a DS3 constant current isolated stimulator (Digitimer LTD., Welwyn Garden City, UK)) of the motor cortex using two percutaneously placed 30G stainless steel stimulation electrodes. Responses were recorded from the gastrocnemius muscle using $30 \mathrm{G}$ platinum transcutaneous needle electrodes (distance between recording electrodes $\sim 1$ cm; Grass Technologies, Astro-Med, Inc., West Warwick, RI, USA). Recording electrodes were connected to an active headstage (3110W Headstage, Warner Instruments LLS) and signal amplified using DP-311 differential amplifier (Warner Instruments LLS). Amplified signal was acquired by the PowerLab 8/30 data acquisition system (AD Instruments, Inc., Colorado Springs, CO) at sampling frequency of $20 \mathrm{kHz}$, digitized and stored in PC for analysis. MEPs were measured until three to five highest (stable) recorded potentials were similar. Those traces were averaged per animal and multiplied by one thousand $(\mu \mathrm{V}$; all values $>1)$. Next, for data normalization, a logarithmical transformation was applied for further analysis (amplitudes of MEP traces tended to vary much more in animals with higher MEPs amplitudes).

\section{Measurement of muscle spasticity}

At 1.5 weeks and 2 months post-injury, the presence of muscle spasticity in the lower extremities was measured using a previously described system (Marsala 2005). Briefly, animals were placed in a restrainer and a hindpaw was taped to a rotational metal plate driven by a computer-controlled 
stepping motor. The metal plate is interconnected loosely to the "bridging" digital force transducer (LCL454G, 0-454 g range; Omega, Stamford, CT, USA). The resistance of the ankle to dorsiflexion was measured during stepping motor-driven ankle dorsiflexion (40 ; MDrive 34 with onboard electronics; microstep resolution to 256 microsteps/full step; Intelligent Motion Systems, Marlborough, CT, USA) at 3 different ankle-rotational velocities $(40,60$ or $80 \%$ sec). The EMG signal was recorded from the ipsilateral gastrocnemius muscle during the same time frame. To record EMG activity, a pair of tungsten electrodes was inserted percutaneously into the gastrocnemius muscle $1 \mathrm{~cm}$ apart. EMG signals were bandpass filtered $(100 \mathrm{~Hz}$ to $10 \mathrm{kHz}$ ) and recorded before, during, and after ankle dorsiflexion. EMG responses were recorded with an alternating current-coupled differential amplifier (model DB4; World Precision Instruments, Sarasota, FL, USA). EMG was recorded concurrently with ankle resistance measurements, both with a sample rate of $1 \mathrm{kHz}$. Both muscle resistance and EMG data were collected directly to the computer using custom software (Spasticity version 2.01; Ellipse, Kosice, Slovak Republic). Each recorded value was the average of three repetitions. The presence of spasticity response was identified as an increased ankle resistance and concurrent increase in recorded EMG activity during computer-controlled ankle dorsiflexion. To measure the contribution of "mechanical" component in measured resistance (i.e., caused by ankle ankylosis in chronically paraplegic animals), animals were anesthetized with isoflurane at the end of each recording session and the relative contribution of neurogenic (i.e., isoflurane-sensitive) and mechanical (i.e., isoflurane non-sensitive) component identified. The magnitude of antispasticity effect after any manipulation was then expressed as the maximum possible anti-spasticity effect measured under isoflurane anesthesia minus the value of the mechanical component.

\section{Sensory testing}

Recovery of sensory function was assessed through quantification of supraspinal "above-level" escape response (AL-ER; i.e., an escape or escape-attempt with incorporation of the forelimbs) thresholds to 1 ) a gradually increasing force to the hind paws (using the Analgesy-Meter, no disc weights added; Cat\# 37215, Ugo-Basile, Collegeville, PA, USA), and 2) AL-ER latencies to a constant heat stimulus (intensity 17, cut-off at $30 \mathrm{sec}$ ) to the hind paws (using an constant infrared heat source; Cat\# 37360, Ugo-Basile, Collegeville, PA, USA). The hind paw tested was gently restrained by the investigator to prevent withdrawal. For the heat perception test the apparatus was switched on $\geq 15$ min prior to testing, to allow it to warm up.

For the AL-ER tests, both hind paws were tested four times, alternately, for each test, with a testing interval of $\geq 1$ hour. No more than four measurements per day were performed, rendering two testing days per test. Maximum cut-off values for the stimuli or latency were at approximately two 
times that of response threshold of uninjured animals, to prevent tissue damage. Prior to (one week) and during the experimental period, the animals are extensively habituated to the experimenter so that the animals can be held upright (loosely) during all sensory assessments. Habituation consists of picking the animal up and holding/handling it twice daily for $\geq 3$ minutes. Subsequently, in absence of a stimulus, animals did only rarely show escape behavior when held for the time it would take to reach cut-off values. We measured the AL-ER thresholds/latencies before injury (baseline) and every $2^{\text {nd }}$ week after injury. The final measurement was done at eight weeks post-injury. Two or less (out of the total of eight, bilateral) measurements could manually be assigned as outliers and be excluded per time point (done while blinded for time point, animal, and treatment group). In addition, individual scores were log transformed before analysis and we calculated the Maximal Possible Effect, using these log scores, as previously suggested (Mills 2012). Hence, we used the standard formula to calculate the Maximal Possible Effect, and assuming a logarithmic relation between stimulus intensity and perceived intensity:

$$
100 \times \frac{\log \left(x_{\text {final }}\right)-\log \left(\bar{x}_{\text {final of SCCI control animals }}\right)}{\log \left(\bar{x}_{\text {baseline of SCCI animals }}\right)-\log \left(\bar{x}_{\text {final of SCCI control animals }}\right)}
$$

Here, $x_{y}$ is the average AL-ER threshold of an individual animal at time point $y$ (either for a thermal or mechanical stimulus).

\section{Magnetic resonance imaging}

Eight weeks after cell grafting, rats were deeply anesthetized with $2 \mathrm{mg}$ pentobarbital and $0.25 \mathrm{mg}$ phenytoin ( $0.5 \mathrm{~mL}$ of Beuthanasia-D, Intervet/Schering-Plough Animal Health Corp., Union, NJ, USA) and transcardially perfused with $200 \mathrm{ml}$ of heparinized saline followed by $250 \mathrm{ml}$ of $4 \%$ paraformaldehyde (PFA) in PBS. A $3 \mathrm{~cm}$ piece of the vertebral column (Th8-L1) was placed in a tight small latex container filled with 4\% PFA to prevent the formation of air bubble/tissue interface artifacts. Samples were scanned using Magnetic Resonance Imaging (MRI). Images were acquired using a 7T Bruker (Bruker Biospin Billerica, MA) horizontal bore small animal magnet, and a $2.5 \mathrm{~cm}$ imaging volume transmit/receive coil. A 3D turboRARE sequence was used with the following imaging parameters: Echo time/Repetition time 45/1500 ms, Flip angle 180 degrees, field of view 16 $\times 16 \times 16 \mathrm{~mm}$, matrix $256 \times 256 \times 70$ with a resulting voxel size of $62 \times 62 \times 229$ microns. The imaging time was $84 \mathrm{~min}$ per sample.

Volume reconstructions and calculations were done using Amira software (Visage Imaging GmbH, Berlin, Germany). 


\section{Axon counting in plastic semi-thin sections}

After MRI imaging, spinal cords were dissected from the spine, transverse (1.5-mm-thick) spinal cord block cut from the injury epicenter, was then removed from the spinal cord and prepared for plastic embedding as previously described (Navarro). Briefly, dissected tissue blocks were treated with $0.1 \%$ osmium tetroxide in $0.1 \mathrm{M}$ non-saline phosphate buffer $(\mathrm{pH}$ 7.4) for $12 \mathrm{~h}$, followed by adequate rinsing in non-saline phosphate buffer. This was followed by progressive alcohol dehydration according to standard procedures up to $100 \%$ ethanol, with the addition of further dehydration in a 1:1 solution of ethanol/propylene oxide, and lastly in 100\% propylene oxide. Dehydrated blocks were then prepared for resin infiltration by incubation in a 1:1 solution of resin/propylene oxide on a rotator in a fume hood overnight. The resin solution used consisted of: Eponate 12, Araldite 502, dodecenyl succinic anhydride, and 2,4,6-tri [dimethylamino-methyl] phenol (DMP-30; Ted Pella, Inc., Redding, CA), mixed in ratios of 10:10:25:1, respectively. The blocks were then transferred to $100 \%$ resin for subsequent overnight infiltration on a rotator. Finally, the tissue blocks were embedded using fresh resin in multi-chamber silicone rubber molds made from a Silastic ${ }^{\circledR}$ E RVT Silicone Rubber Kit (Dow Corning Corp., Midland Township, MI). The molds with embedded sections were placed in an oven at $60^{\circ} \mathrm{C}$ for 1 day to facilitate resin polymerization. Semi-thin $(1 \mu \mathrm{m})$ transverse sections were then cut using a microtome (Leica Supercut RM 2065) with a 8-mm diamond knife (Histo Diamond Knife, Cat\# LM 7045, DiATOME, Hatfield, PA, USA). The sections were mounted on slides with distilled water and allowed to dry on a slide warmer. Prior to staining, the slides were incubated at $60^{\circ} \mathrm{C}$ in an oven for $10-15 \mathrm{~min}$, and then contrast-stained with $4 \%$ para-phenylene-diamine (PPD).

Mosaic images were taken of two sections per animal at 20X using a Zeiss Imager.M2 fitted with a Zeiss MRm camera (Carl Zeiss Microscopy, Thornwood, NY, USA), a BioPrecision2 stage (Cat\# 96S100, Ludl Electronic Products, Hawthorne, NY, USA), and Stereo Investigator software (MBF Biosciences, Williston, VT, USA). Complete mosaic images were loaded into ImageJ 1.45s. Axonal quantification involved manual definition of pixel threshold (0-255, grayscale; using the Triangle method). Next, ImageJ's Analyze Particles option was used to find particles with a size of 0.20-250 $\mu \mathrm{m}^{2}$ and a circularity of 0.5 to 1.0 (which corresponded to axons). All acquisition and analysis values were held consistent throughout the study. Final measurements acquired were the minimal diameter (Feret's) of each particle (and particle counts). Particles with a minimum diameter $>10 \mu \mathrm{m}$ were excluded. Employment of this parameter allowed for further axonal analysis, in which axons were divided into empirically-derived caliber sizes of small, medium, and large axons (0.3-1.0 $\mu \mathrm{m}$, 1.0-2.5 $\mu \mathrm{m}$, and 2.5-10 $\mu \mathrm{m}$, respectively). Data was acquired per spinal region (i.e., dorsal, ventral, and lateral funiculi). 


\section{Immunofluorescence staining}

After removing the $1.5 \mathrm{~mm}$ block from the spinal cord at the injury epicenter, the remaining caudal and rostral parts of the spinal cord ( $\pm 1 \mathrm{~cm}$ each) were placed in $30 \%$ sucrose for cryoprotection for a minimum of 5-7 days. Transverse spinal cord sections were then prepared from the $L 6$ segment. The segment(s) in between the L6 and the injury epicenter and the one rostral to the injury epicenter were sectioned coronally and used for identification of grafted human cells. All sections were cut on a cryostat and stored free-floating in PBS with thimerosal (0.05 wt\%).

Sections were stained overnight at $4^{\circ} \mathrm{C}$ with primary human-specific $(\mathrm{h})$ or non-specific antibodies in PBS with 0.2\% Triton X-100: mouse anti-Nuclear Mitotic Apparatus (hNUMA, 1:100; Millipore, Billerica, MA, USA), mouse anti-Neuron Specific Enolase (hNSE, 1:500; Vector Labs, Burlingame, CA, USA), mouse anti-Synaptophysin (hSyn, 1:2,000; Millipore), rabbit anti-Glial Fibrillary Acidic Protein (hGFAP, 1:500; Origene, Rockville, MD, USA), mouse anti-Neuronal Nuclei (NeuN, 1:1,000; Millipore), chicken anti-Green Fluorescent Protein (GFP, 1:1,000; Aves Labs, ), rabbit anti-Anti-Glutamate Decarboxylase 65 \& 67 (GAD65\&67; 1:300; Millipore), mouse anti-GFAP (Cy3-labeled; 1:500; SigmaAldrich; St. Louis, MO, USA), rabbit anti-Ki67 antibody (mitotic marker, 1:100; Abcam), and rat anti human axonal neurofilament antibody (hHO14; 1:100; gift from Dr. Virginia Lee; University of Pennsylvania). Mouse anti-Growth Associated Protein 43 (GAP43, 1:16,000; Millipore), rabbit antiCalcitonin Gene-Related Peptide (CGRP, 1:1,000; Biotrend, Destin, FL, USA), and rabbit anti-lonized calcium Binding Adaptor molecule 1 (Iba1, 1:1,000; Wako, Richmond, VA, USA), were used on the L6 transverse sections. Following washing in PBS $3.5 \mathrm{~min}$, sections were incubated with fluorescentconjugated secondary donkey antibodies (Alexa ${ }^{\circledR}$ Fluor 488 \& 647; 1:500; Jackson Immuno Research, West Grove, PA, USA; \& Alexa ${ }^{\circledR}$ Fluor 555, 1:500; Invitrogen). Sections were then mounted on slides, dried at room temperature, and covered with Prolong anti-fade kit (Invitrogen Corp., Carlsbad, CA, USA).

Confocal images (1024 × 1024 pixels) were captured with a Fluoview FV1000 microscope (Olympus, Center Valley, PA, USA) with a 20X or 40X objective, optical section spacing of $0.5 \mu \mathrm{m}$, and pulse speed of $20 \mu \mathrm{sec} / \mathrm{pixel}$. Other images were taken using the Zeiss Imager.M2 setup as described above, using a 10, 20 or $63 \mathrm{X}$ magnification. CGRP, GAP43, and lba1 stainings on L6 transverse sections were quantified using densitometry measurements of the main dorsal horn region (Laminae I through IV; area as marked in Fig. VI-7B). ImageJ software was used for quantification by using the Background Subtraction function. 


\section{Statistical analyses}

Behavioral data were analyzed using ANOVA (one-way, or two-way group $\times$ time repeated measures, using a fixed-effect model, and a Bonferroni post hoc test for multiple comparisons). A $P$ value of 0.05 was considered significant. Unequal variances were explored prior to using ANOVA analyses using the Bartlett's test, but were not identified. Post hoc tests were only calculated if overall group differences were found. Results are expressed as means with the standard error of the mean (SEM). To analyze differences between the two groups (e.g., vehicle injected vs. non-injected $\mathrm{SCl}$ animals), we used Student's t-tests (unequal variances were explored with the F-test, but not found) or repeated measures ANOVA. Naïve and sham operated animals were grouped (and named "noninjured") in all outcomes besides the sensory tests. All statistical analyses were done using GraphPad Prism (La Jolla, CA, USA), SPSS statistics 17 (for K-Means clustering; IBM, Armonk, NY, USA), or STATA 12 (for precise post-hoc test $P$-value calculations; StataCorp LP, College Station, TX, USA) and performed two-tailed.

\section{Results}

\section{General animal health and survival of animals during long-term immunosuppression}

From the total of $35 \mathrm{SCl}$ Sprague-Dawley rats employed in this study, 32 survived until planned sacrifice while continuously immunosuppressed; 14 NSI-566RSC-injected (1 excluded; because of automutilation of hind paw on day two post-injury), 10 vehicle-injected, 8 non-injected ( 2 excluded; 1 because of automutilation of hind paw on day 7 post-injury \& 1 because of excessive BW loss on post-injury day 18 (likely related to immunosuppression-related toxicity)). In four surviving animals, lower extremity ulcers developed but were effectively treated with local standard triple antibiotic ointment (Bacitracin, Neomycin, and Polymyxin B) and cohesive bandages. In most animals, the Crede's maneuver needed to be performed for 3-5 days after spinal trauma (exceptions: 3 animals in NSI-566RSC-injected group and 2 animals in non-injected SCl-control group, of which one died due to health issues; see above). No additional worsening (i.e., a lowering in open-field locomotor scores at one day post-grafting, compared to pre-grafting values) was noted in intraspinal media- or cellinjected animals.

\section{Spinal injection procedure did not alter neurological outcome in previously L3-contused rats}

In order to define the effect of spinal injection itself in modulating the functional recovery profile (i.e., potential worsening in neurological outcome) in L3-injured animals, we have first compared the effect of spinal media injection only with spinal injury animals which received no injections (10 vehicle-injected and 8 non-injected $\mathrm{SCl}$ animals). No significant differences were found between 
these two groups in any of neurological or electrophysiological outcome measures used in this study (repeated measures ANOVA for open field locomotor scores; Student's $t$-test for others). Based on these data, which showed no significant differences between both control groups, these two groups were then pooled into one control group and used for subsequent comparison with HSSC-grafted animals.

\section{Assessment of motor function}

Gait analysis was conducted at 8 weeks after grafting (or corresponding time point in controls) using the Catwalk apparatus (Koopmans 2005). The following parameters were analyzed: 1) runway crossing time, 2) rostro-caudal hindpaw positioning, 3) hind paws base of support, 4) regularity index/coordination, 5) stride length, and 6) phase dispersions.

Rostro-caudal hindpaw positioning (RCHPP): In control non-injured animals, the RCHPP was $0 \pm 1.7$ $\mathrm{mm}$ (i.e., the animals are able to achieve a near complete overlap in the hindpaw positioning relative to the last ipsilateral frontpaw print; full rostro-caudal overlap is represented by a value of "0"). Rats receiving spinal HSSC grafts showed significantly better RCHPP, when compared to control SCI animals ( $-9.0 \pm 1.9$ vs. $-18.2 \pm 3.1 \mathrm{~mm}$, respectively, Fig. VI-2A; Bonferroni: $P=0.04)$. Examples of the paw positioning are shown in Fig. VI-2B for a non-injured control, SCl control, and a HSSC-treated SCI animal (Fig. VI-2-B1, -B2, and -B3, respectively).

No significant differences were detected in other Catwalk parameters (runway crossing time, hind paws base of support, regularity index/coordination, stride length, phase dispersions), myogenic motor evoked potentials, or behavioral motor tests (open field locomotor score (modified BBB score, and regular BBB scores), single-frame motion analysis or ladder climbing test) (Fig. VI-8A-D).

To identify the presence of spasticity (i.e., potentiation in muscle stretch-evoked EMG activity) in animals after $\mathrm{SCl}$, a computer-controlled ankle-rotational force was applied on the right or left paw in fully awake restrained animals and resulting change in EMG activity in the gastrocnemius muscle and correlative ankle resistance was measured (Marsala 2005).

Independent of $\mathrm{SCl}$ group (control or HSSC-injected), two quantitatively different EMG patterns and corresponding resistance response (EMG/RES) patterns were recorded in spinally-injured animals. First, if compared to control non-injured animals, little or no change in EMG/RES response was seen at 1.5 weeks after $\mathrm{SCl}$. Second, $\mathrm{SCl}$ induced an increased spasticity response in a portion of the animals at 1.5 weeks after injury. A K-Means clustering method was used to group all 44 ( $\mathrm{SCl}$ and non-injured) animals into 2 groups based on magnitude of resistance to ankle rotation at 1.5 weeks post-injury (or equivalent time point in non-injured animals). 
A Catwalk: Hind paw positioning
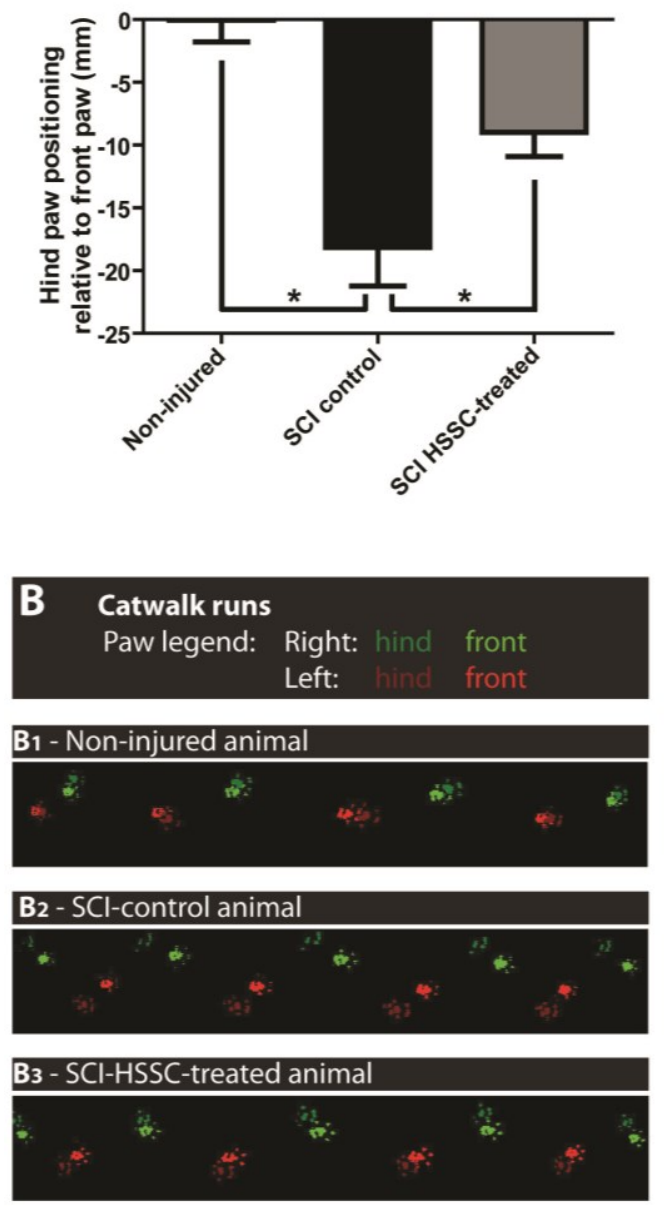

C Relative change in spasticity
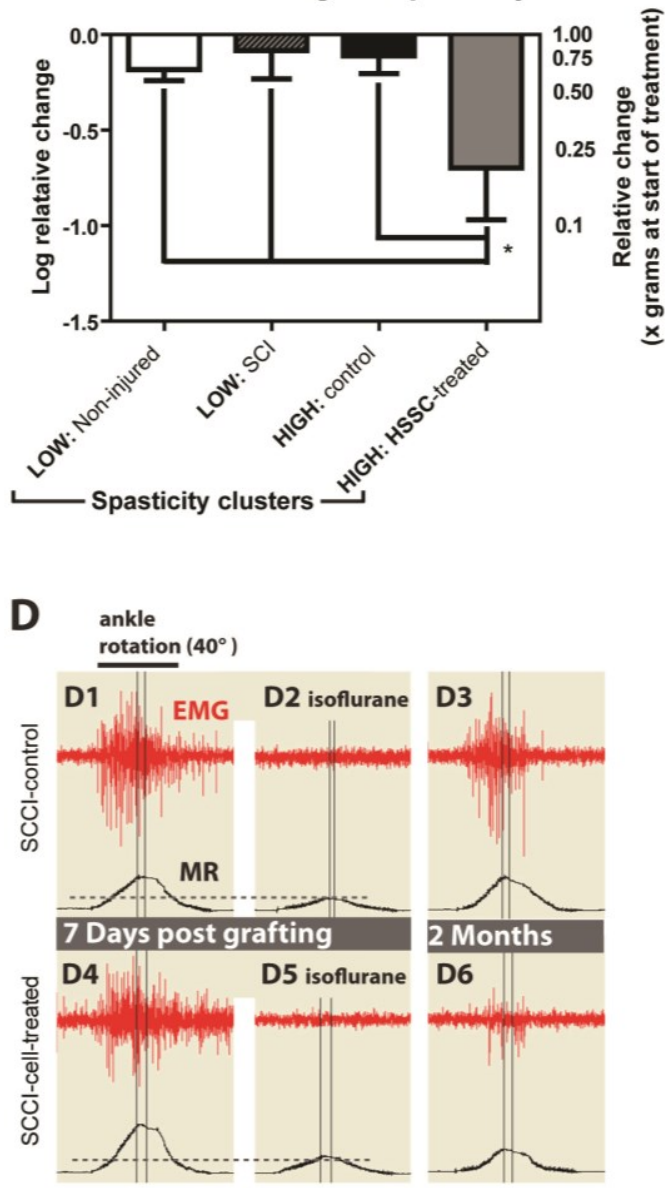

Figure VI-2: Improvement in paw positioning and muscle spasticity. A: The gait analysis of hind paw positioning at 2 months after treatment. In comparison A significant improvement was seen in HSSCgrafted animals. B1-B3: Example of the sequence of paw step images in naïve (B1) SCl-control (B2) and SCI-HSSC-treated animals (B3). Note a near complete paw footprint overlap between the front and the hind paws in naïve animals (B1) but a substantial dissociation in the paw footprint overlap in SCl controls (B2). An improvement in paw footprint placement in SCI-HSSC-treated animals can be seen (B3). C: Analysis showed significant suppression of spasticity response (expressed as a muscle resistance ratio: values at 2 months vs. 7 days post injury in "HIGH spasticity" HSSC-treated animals if compared to "HIGH spasticity" controls. D: To identify the presence of muscle spasticity in fully awake animals, the hind-paw ankle is rotated $40^{\circ}$ at a velocity of $80^{\circ} / \mathrm{sec}$. The spasticity response is identified by exacerbated EMG activity measured in the gastrocnemius muscle and corresponding increase in muscle resistance. In control SCl animals with developed spasticity (i.e., "high spasticity"/HIGH group), no change in spasticity response if compared to 7 days post-vehicle injection was seen at 2 months (compare D1 to D3). In contrast, a clear decrease in spasticity response was seen in SCI-HSSC-treated animals at 2 months after cell injections (compare D4 to D6). To identify the presence of mechanical resistance, animals are anesthetized with isoflurane at the end of the recording session and the contribution of mechanical resistance (i.e., isoflurane nonsensitive) is calculated. (D2, D5: data expressed as mean士SEM; one-way ANOVAs). 
Seven animals of each $\mathrm{SCl}$ group (i.e., control or HSSC-injected) were found to be clustered in the high "spasticity" group (HIGH), which had a $31.7 \pm 3.9 \mathrm{~g}$ increase in measured muscle resistance during ankle rotation, compared to the low "spasticity" group (LOW) showing $8.9 \pm 1.5$ g resistance (Student's t-test: $P<0.0001)$. No difference in the incidence of this high "spasticity" response was noted between SCI control vs. cell-treated groups (incidence: $X^{2}: P=0.53$; extend: Student's $t$-test: $P=0.24)$. No naïve or sham operated animals were found to be clustered into the HIGH group. Resistance to ankle rotation measured 8 weeks after treatment (and expressed as relative change from 1.5 weeks post-injury values) showed a significant decrease in the HSSC-injected HIGH resistance group when compared to HIGH resistance animals from the control SCI group (Fig. VI-2C; i.e., decline of $24.8 \pm 6.4 \mathrm{~g}$ in $\mathrm{HSSC}$-injected animals and $4.8 \pm 6.3$ in control $\mathrm{SCl}$ animals; Bonferroni: $P=0.048)$.

Fig. VI-2D shows an example of raw data depicting a post injury EMG response (red channel) and corresponding increase in muscle resistance (black channel) during ankle rotation in a SCl-control (Fig. VI-2-D1-3) and a HSSC-injected animal (D4-6) at 7 days after treatment and the end of the 8week survival. Clear suppression of potentiated EMG response and muscle resistance can be seen in HSSC-treated animals (compare D4 to D6). To identify and dissociate neurogenic (i.e., isofluranesensitive) versus mechanical (i.e., isoflurane non-sensitive) components, muscle resistance was remeasured after isoflurane anesthesia and the relative contribution of mechanical component calculated. The induction of isoflurane anesthesia near completely blocked the ankle rotationevoked EMG response and resulting increase in muscle resistance (D2, D5).

\section{Assessment of sensory functions}

Analysis of mechanical and thermal sensory function was performed by comparing hindpaw thresholds improvements of evoked above-level/supra-spinal withdrawal responses (i.e., an escape response in which the frontlimbs and/or vocalizations are used) between experimental groups over several time points. Groups consisted of naïve control, sham operated control, SCl-control, or SCIHSSC-injected animals. Response thresholds were measured before injury and every second week thereafter. No differences were measured between naïve and sham-operated animals at any time point in response thresholds to both mechanical and thermal stimuli (repeated measures ANOVA).

Prior to injury, no differences in mechanical thresholds to trigger escape responses were measured between all four experimental groups (on average $92 \pm 2 \mathrm{~g}$ ). After $\mathrm{SCl}$, the thresholds increased significantly in both SCl-control and SCl-HSSC-injected animals compared to control non-injured groups, at all time points (Bonferroni; $P<0.001$ ). From 4 weeks post injury, $\mathrm{SCl}-\mathrm{HSSC}$-injected animals displayed a trend towards progressive improvement in response thresholds if compared to $\mathrm{SCl}$ 
controls (at eight weeks: $177 \pm 10 \mathrm{~g}$ and $216 \pm 10 \mathrm{~g}$, respectively; Fig. VI-3A; repeated measures ANOVA: $P=0.14)$. This resulted in a significant higher percentage of the Maximal Possible Effect for improvement of mechanical stimulus perception in SCl-HSSC-injected animals compared to SCl-control animals (Fig. VI-3C; Student's $t$-test: $P=0.03$ ).

Prior to $\mathrm{SCl}$, measurement of thermal (infrared) stimulus-evoked paw withdrawal threshold showed no significant differences between all experimental groups $(17.3 \pm 0.3 \mathrm{sec}$; one-way ANOVA). At two weeks post $\mathrm{SCl}$, significant increase in paw withdrawal latencies in both the control $\mathrm{SCl}$ group and in $\mathrm{SCl}$ animals receiving spinal HSSC grafts were measured, when compared to control non-injured (sham operated and naive) groups $(26.1 \pm 0.7 \mathrm{sec}$ in $\mathrm{SCl}$-control animals and $26.5 \pm 0.7 \mathrm{sec}$ in HSSC-grafted animals vs. $18.3 \pm 0.2 \mathrm{sec}$ in control non-injured animals;

Fig. VI-3B; Bonferroni: $P<0.001)$.

From four weeks after treatment a trend towards a progressive normalization in response threshold was seen in HSSC-treated animals if compared to $\mathrm{SCl}$ controls (at eight weeks: $24.0 \pm 0.9 \mathrm{sec}$ in $\mathrm{SCl}-$ control and $21.4 \pm 0.9 \mathrm{sec}$ in HSSC-injected animals, respectively; repeated measures ANOVA: $P=0.09$ ). This resulted in a significantly higher percentage of the Maximal Possible Effect for the improvement of thermal stimuli in SCI-HSSC-injected animals compared to SCl-control animals (Fig. VI-3C; Student's $t$-test: $P=0.02)$.

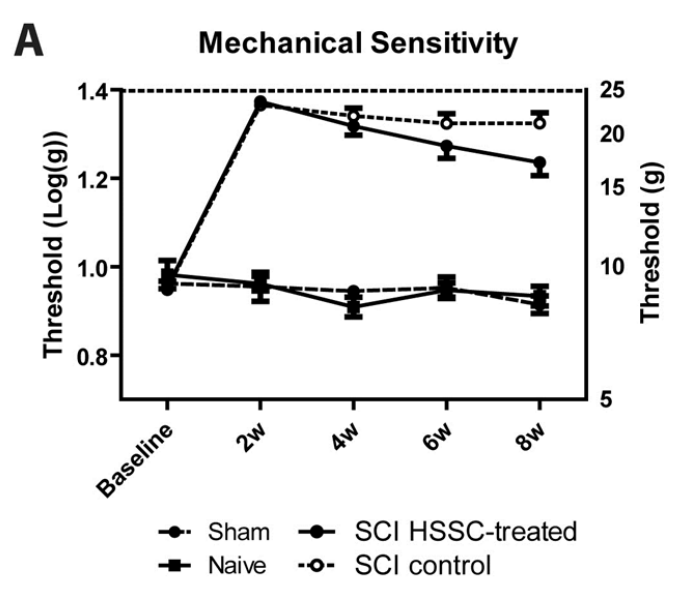

B Thermal Sensitivity

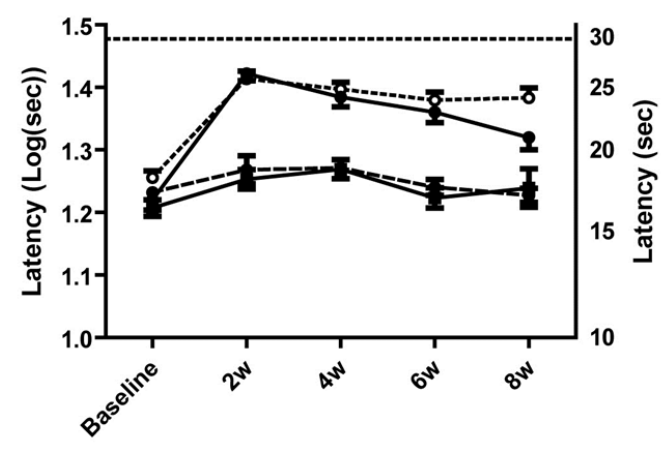

C Sensory Improvement

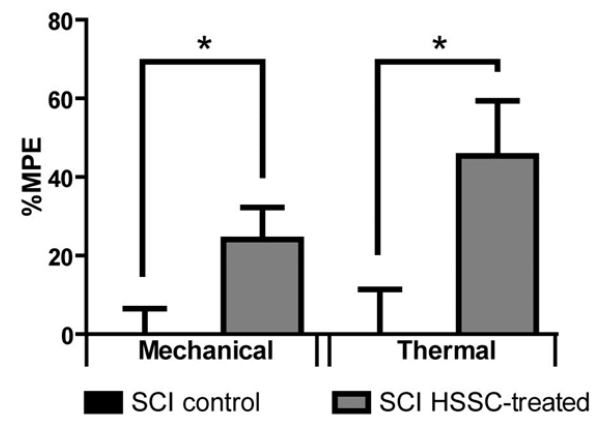

Figure VI-3: Amelioration of hypoesthesia in SClHSSC-grafted animals. A \& B: Baseline and biweekly assessments of perceptive thresholds for (A) mechanical and (B) thermal stimuli, applied below the level of injury, showed a trend towards progressive recovery in SCI-HSSC-grafted animals. C: When expressed as percentages of the Maximal Possible Effect for mechanical and thermal perceptive thresholds improvements, SCI-HSSCtreated animals showed significant improvements in sensory function for both mechanical and thermal components. (A-C: data expressed as mean $\pm S E M$; AB: repeated measures ANOVAs; C: Student t-tests). 
Postmortem spinal cord MRI showed cavity-filling effect by grafted cells in HSSC-injected animals

For lesion volume analyses, a $2 \mathrm{~cm}$ long portion of the fixed spinal column was dissected out, kept in 4\% paraformaldehyde, and imaged using a 7 TESLA MRI magnet. The primary goal of this analysis was to generate quantitative data on the cavity-filling effect by grafted cells and to assess the extent of rostro-caudal cavitation in vehicle-injected vs. HSSC-injected animals using quantitative volume analysis (Fig. VI-4). In vehicle-injected animals, the presence of fluid-filled cavities was readily identified as the presence of homogenous white areas and scarring as black areas (Fig. VI-4B1; compare with non-injured: Fig. VI-4C). In contrast, in animals receiving cell injection, the cavity was partially or completely filled with grafted cells as evidenced by the presence of low density tissue masses (Fig. VI-4A1). The identity/presence of grafted cells in the "low density tissue masses" was further validated by analysis of semi-thin plastic sections taken from the same region (compare Fig. VI-4A2 which depicts the presence of cell grafts vs. extensive cavity in Fig. VI-4B2). Fig. VI-4A shows a 3D reconstruction image of a cell injected animal (areas identified as grafted cells are labeled green). Fig. VI-4B shows a SCl-control (media-injected) animal with cavity labeled in light-greenyellow.

Quantification of the cavity volume showed a significantly larger injury-induced cavity in SCl-control animals than in HSSC-injected animals $\left(3 \pm 0.4 \mathrm{~mm}^{3}\right.$ vs. $0.6 \pm 0.2 \mathrm{~mm}^{3}$, respectively; Fig. VI-4D; Student's $t$-test: $P<0.0001)$. Similarly, the scar volume seen in $\mathrm{SCl}$-control animals was larger than in cell-injected animals $\left(3.3 \pm 0.3 \mathrm{~mm}^{3}\right.$ vs. $1.9 \pm 0.3 \mathrm{~mm}^{3}$, respectively; Student's $t$-test: $\left.P<0.001\right)$.

To assess the potential excessive grafted cell proliferation and resulting spinal cord tissue expansion, we next compared the total volume (i.e., the volume of the remaining spinal cord, scar, cavity, and/or grafted cells) of cell-grafted spinal cord segments with corresponding segments of control animals. The measured volumes were: $71.8 \pm 3.2 \mathrm{~mm}^{3}$ in non-injured control animals, $54.6 \pm 2.8 \mathrm{~mm}^{3}$ in SCl-control animals, and $59.0 \pm 2.2 \mathrm{~mm}^{3}$ in SCl-HSSC-injected animals (Student's $t$-test: $P=0.27 ; \mathrm{SCl}$ control vs. SCI-HSSC-injected animals). 

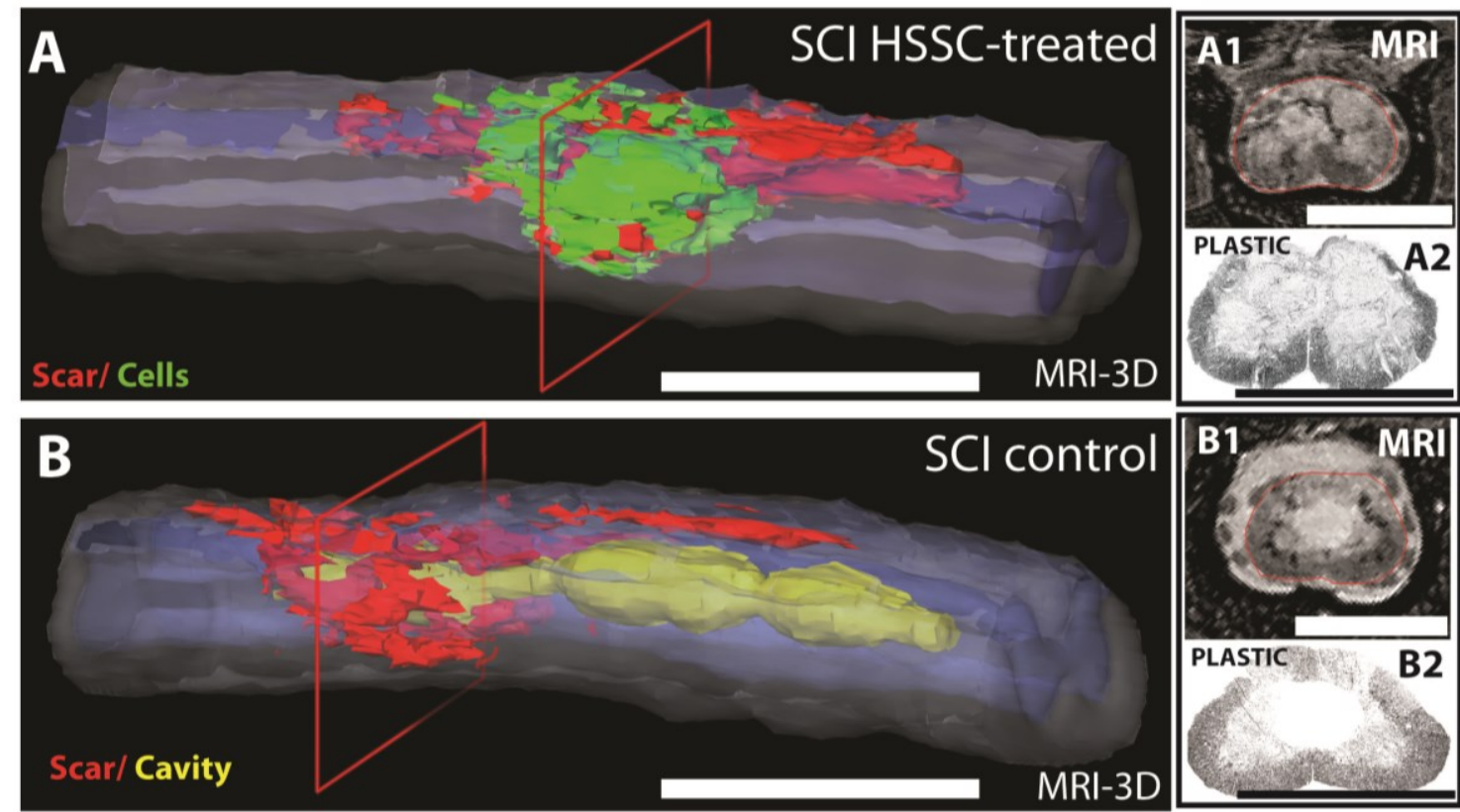

\section{Changes in MRl-defined cavity and scar volume}
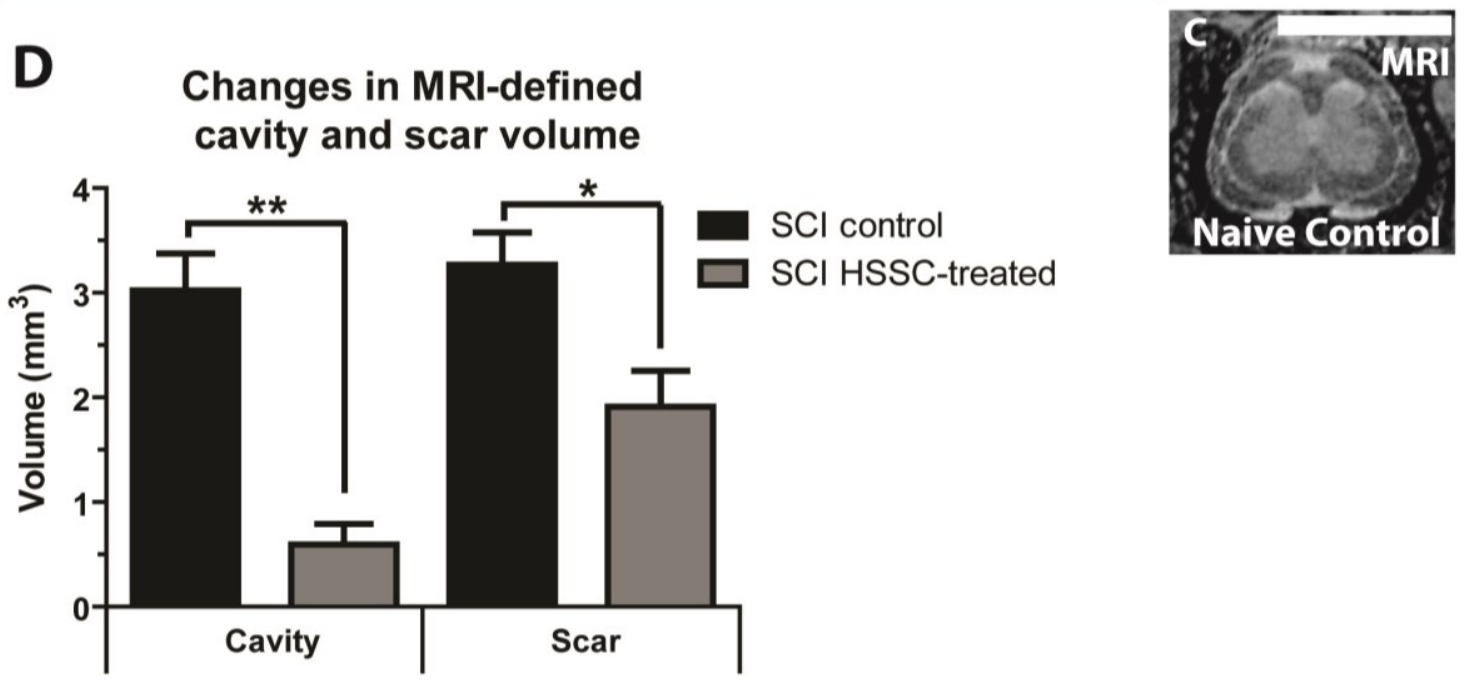

Figure VI-4A-D: Effective cavity-filling effect by transplanted cells in SCI HSSC-injected animals. At the end of the 2-month post-treatment survival, animals were perfusion fixed with $4 \%$ paraformaldehyde, spinal column dissected and MRI-imaged in situ before spinal cord dissection for further histological processing. A, B: 3-D MRI images of spinal cord segments in animals with previous traumatic injury and treated with spinal HSSC (A) or media (B) injections. Note the near complete injected-cells cavity-filling effect in HSSC-treated animals. A1, A2, B1, B2: To validate the presence of grafted cells or cavitation at the epicenter of injury, the same region was histologically processed, semi-thin plastic sections prepared and compared to corresponding MRI image (compare A1 to A2 and B1 to B2). C: 2-D MRI image taken from naïve-non-injured animal. D: Quantification of the cavity and scar volume from serial MRI images showed a significantly decreased cavity and scar volumes in SCI-HSSC-injected animals if compared to media-injected SCI controls. (D: data expressed as mean $\pm S E M$; Student $t$-tests), (Scale Bars: A, B: 5 mm; A1, A2, B1, B2, C: 3 mm). 


\section{Survival, maturation and integration of grafted HSSC}

To identify the presence of human cells in the rodent spinal cord tissue, two different immunostaining/analytical methods were used. First, GFP-tagged grafted cells were identified by the presence of GFP autofluorescence/immunoreactivity and then co-stained with neuronal and nonneuronal markers. Second, a set of human-specific antibodies was first used to validate the presence of human cells and then combined with other human-non-specific neuronal or non-neuronal antibodies.

Staining with anti-GFP, -NeuN (neuronal marker) and -human-specific synaptophysin antibody showed a near complete repopulation of compression-induced lesion cavity by grafted GFP+ cells (Fig. VI-5A-yellow dotted area). A comparable spinal injury-cavity filling by grafted cells was seen after grafting with GFP or non-labeled HSSC as evidenced by the presence of dense hNUMAimmunoreactive grafts (Fig. VI-5A inserts). Analysis of axo-dendritic sprouting from grafted GFP+ cells showed extensive rostro-caudal neurite sprouting particularly well-developed in the lateral white matter (Fig. VI-5B). In addition, numerous GFP+ axons branching from innervated lateral funiculi and projecting towards $\alpha$-motoneurons and interneurons was identified (Fig. VI-5B; insert). Triple staining with NeuN, hSYN and GFP antibody showed a high density of hSYN punctata in GFP+ innervated regions (Fig. 5C-yellow arrows) as well in the vicinity of endogenous $\mathrm{NeuN}+$ neurons. Staining with hNUMA, hNSE and DCX antibody revealed that the majority of hNUMA+ grafted cells were DCX or DCX/hNSE immunoreactive (Fig. VI-5A-insert; Fig. VI-5D). Probing for glial phenotype in grafted cells by double staining with hNUMA and hGFAP or hNUMA and Olig2 antibody revealed well-developed groups of hGFAP+ astrocytes. These GFAP+ cell populations were primarily found in the white matter or at the periphery of individual DCX/hNSE+ grafts (Fig. VI-5E). Less than $2 \%$ of hNUMA+ cells showed Olig2 immunoreactivity (Fig. VI-5F; yellow arrows). To assess the presence of mitotically active grafted cells, sections were double-stained with hNUMA and Ki67 antibody. An estimated $0.5-1 \%$ of hNUMA+ cells were Ki67 positive. These double hNUMA/Ki67+ cells were regularly distributed throughout the grafted regions but no cluster(s)-like formations of hNUMA/Ki67+ cells were seen in any animal (Fig. VI-5G; yellow arrows).

Confocal analysis of spinal cord sections triple-stained with hSYN, GFP and NeuN antibodies showed numerous hSYN punctata colocalizing with GFP+ processes. Several hSYN punctata were found to reside in the vicinity of interneuronal and/or $\alpha$-motoneuronal membranes (Fig. VI-6A; inserts; white arrows). Probing for the presence of GAD65/67+ terminals derived from grafted neurons by using triple stained GAD(65/67)/GFP/NeuN sections and confocal microscopy showed the presence of GFP/GAD65/67+ terminals in the vicinity of $\alpha$-motoneuronal membranes (Fig. VI-6B; white arrows). 

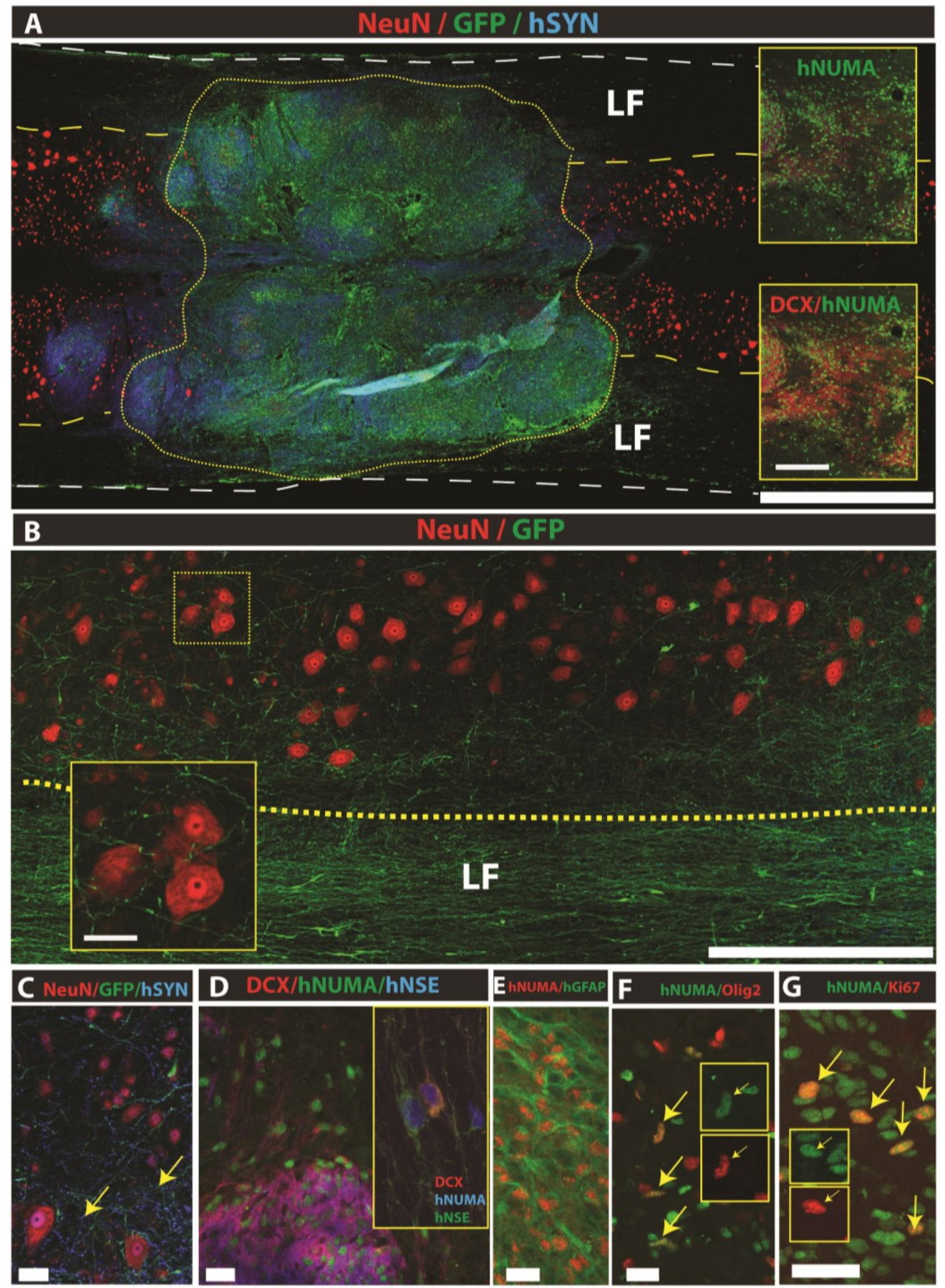

Figure VI-5: Survival, differentiation and outgrowth. A: Grafted cells filling near completely the lesion cavity at 8 weeks after grafting (yellow dotted area; inserts). B: Detail from "A": a dense GFP+ neurite network in the lateral funiculus (LF) and with axons projecting towards $\alpha$-motoneurons and interneurons in the gray matter (insert). C: In areas with a dense GFP+ axodendritic network, hSYN immunoreactivity associated with GFP+ processes can be seen (yellow arrows). D: The majority of grafted cells showed development of the neuronal hNSE/DCX+ phenotype. E, F: A subpopulation of grafted hNUMA+ cells showed astrocyte (hGFAP+) and oligodendrocyte (Olig 2) phenotype (F; yellow arrows). G: Using mitotic marker Ki67, regularly distributed hNUMA/Ki67+ grafted cells were identified (yellow arrows). (Scale Bars: A: 1.5mm (inserts: $200 \mu \mathrm{m}$ ); B: $600 \mu \mathrm{m}$ (insert: $75 \mu \mathrm{m}$ ); C: $60 \mu \mathrm{m}$; D: $20 \mu \mathrm{m}$ (insert: $5 \mu \mathrm{m}$ ); E-G: $10 \mu \mathrm{m}$ ). 

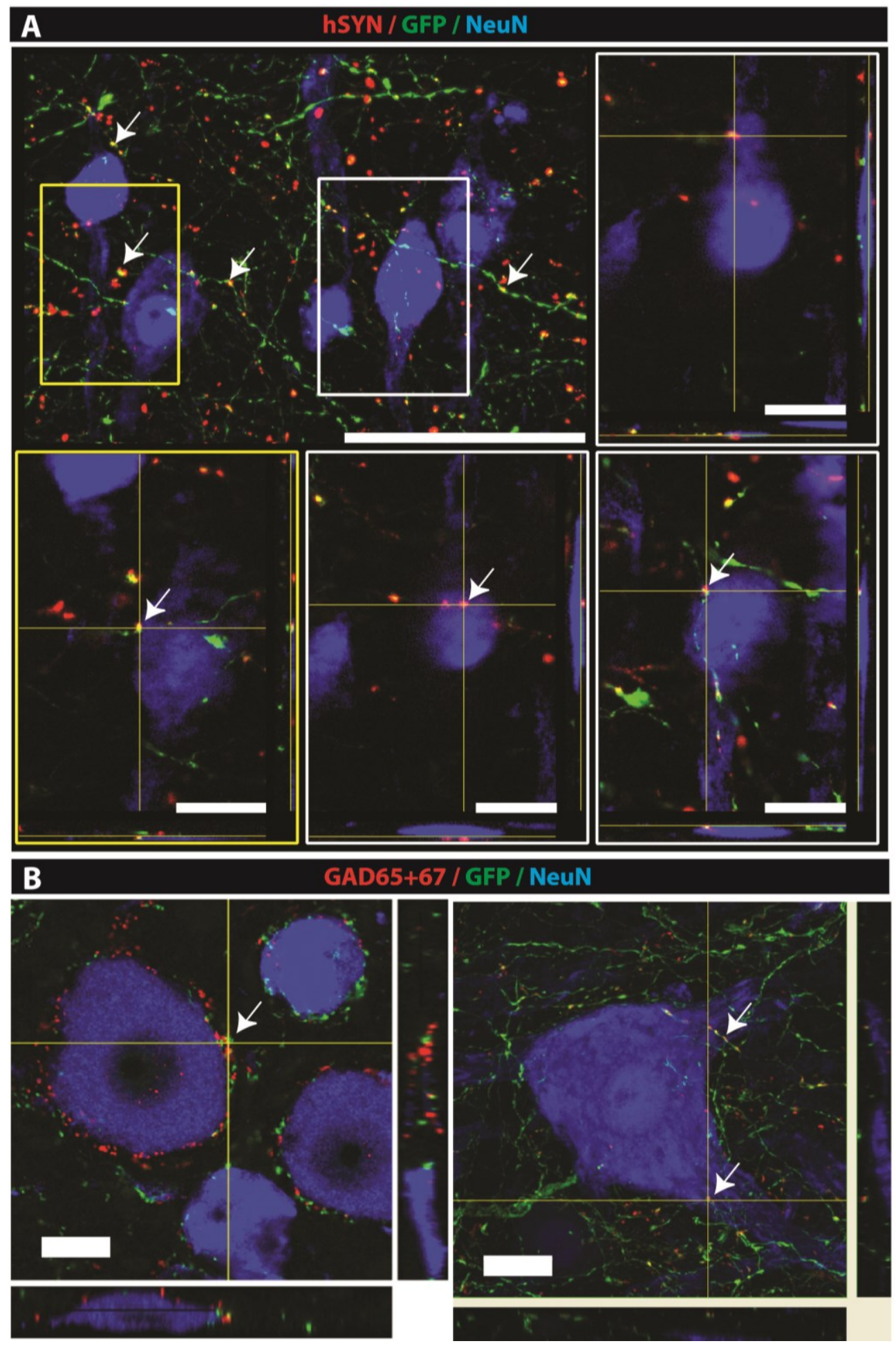

Figure VI-6: Development of putative GABA-ergic synaptic contact between HSSC and the host neurons. A: Confocal analysis of hSN/GFP/NeuN-stained sections shows numerous hSYN punctata associated with GFP+ processes derived from grafted cells. Some of the hSYN/GFP+ terminals were found to be in the vicinity of the host interneurons or $\alpha$-motoneurons (A; inserts; white arrows). B: Triple staining with GAD65+67/GFP/NeuN antibody showed numerous double-stained GAD65+67/GFP+ terminals residing on or in the close vicinity of lumbar $\alpha$-motoneurons (white arrows). (Scale Bars: A: $150 \mu \mathrm{m}$ (inserts: $30 \mu \mathrm{m}$ ); B: 20 $\mu \mathrm{m})$. 

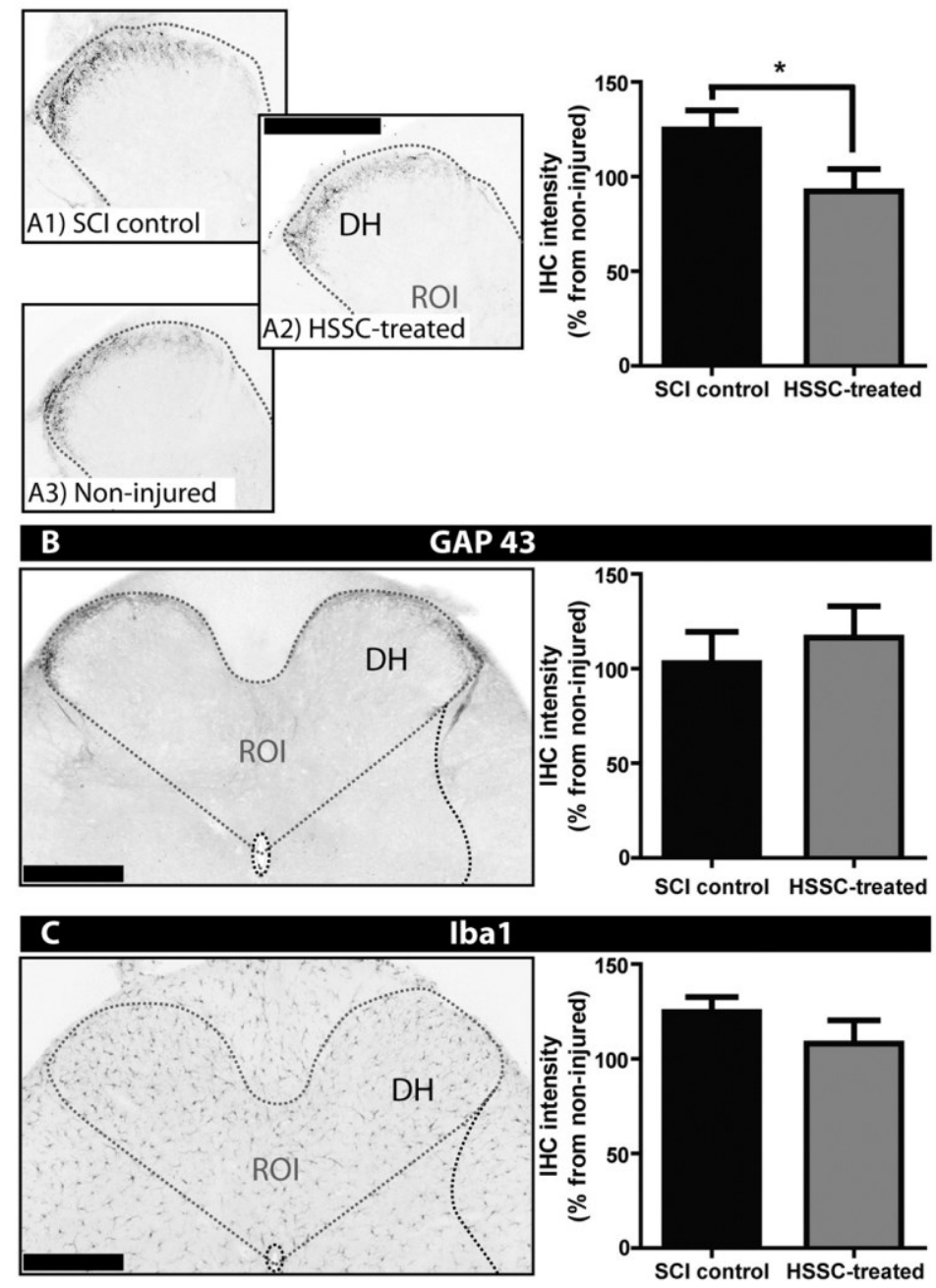

Figure VI-7: Figure 7A-C: Significant decrease in the dorsal horn CGRP immunoreactivity caudal to the injury epicenter in $\mathrm{SCl}$ HSSC-treated vs. SCl-control animals. A-C: CGRP- (A), GAP-43- (B), and Iba1- (C) immunoreactivity in the dorsal horns (DH) caudal of the injury epicenter at 2 months after $\mathrm{L3}$ SCl. The region of interest (ROI) was defined as outlined in B and C (left panels, red dotted line). A: The quantitative densitometry analysis of CGRPimmunostained images in the dorsal horns of SCI-HSSC-treated animals (A2) showed significantly decreased CGRP expression when compared to SCl-control animals (A1). B, C: The dorsal horn GAP-43 or IB1 immunoreactivity was not significantly different between experimental groups. (A-C: data expressed as mean \pm SEM; student $t$-tests). (Scale Bars: A - C: $500 \mu \mathrm{m}$ ).
Normalization of CGRP expression in $\mathbf{L 6}$ dorsal horns in SCl-HSSCtreated animals

To analyze changes in the spinal expression of putative central pain neuromodulators/indicators, including CGRP, GAP43 and Iba-1 (Hains 2006, Ondarza 2003), we next stained transverse 16 sections (i.e., below-injury-level region) with respective antibodies at 8 weeks after treatment (Fig.7 A, B, C). Densitometry analysis showed that CGRP immunoreactivity was significantly reduced in SCl-HSSCtreated animals (Fig. 7A2) when compared to SCl-controls (Fig. 7A1; Student's t-test: $P=0.04)$. We did not find significant group differences in IHC staining intensities of either GAP43 or Iba1 (Fig. 7B, C; Student's $t$-test: $P=0.58$ and $P=0.24$, respectively).

Quantitative assessment of axonal survival in the epicenter of injury using semi-thin plastic sections

For quantitative analysis of axonal survival, a transverse spinal cord block taken from the injury 
epicenter was used. Using osmium/p-phenylenediamine-stained semi-thin (1- $\mu \mathrm{m}$-thick) plastic sections, the total number of axons (divided in 3 subgroups based on the axonal caliber; 0.3-1.0, 1.0-2.5, and 2.5-10 $\mu \mathrm{m}$ in diameter) was then counted using ImageJ software.

A

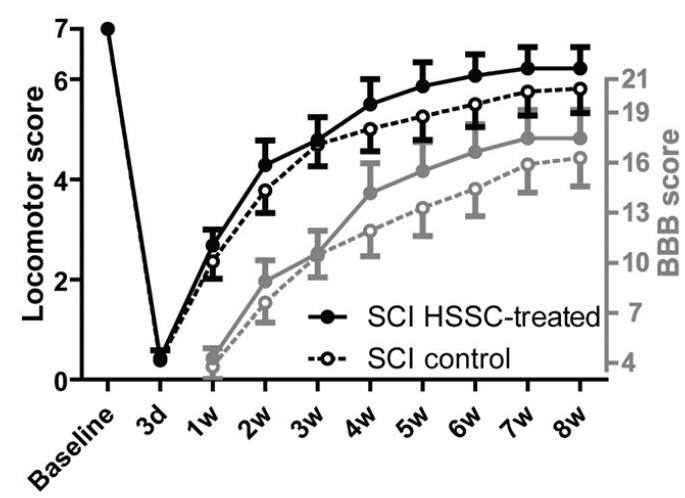

C

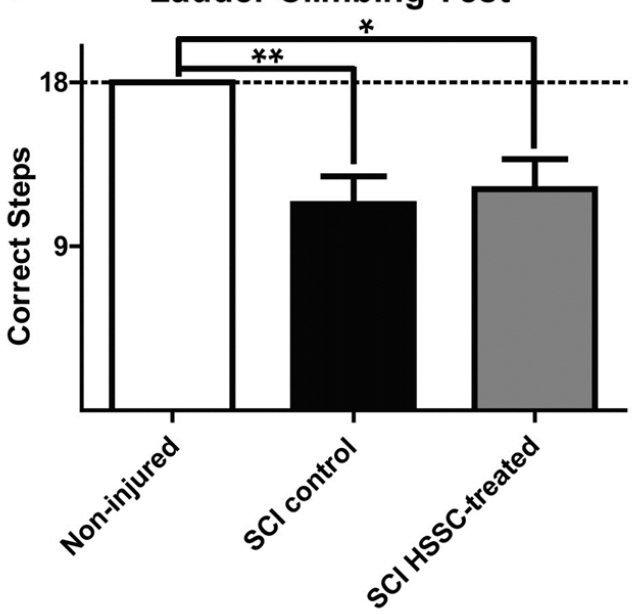

B

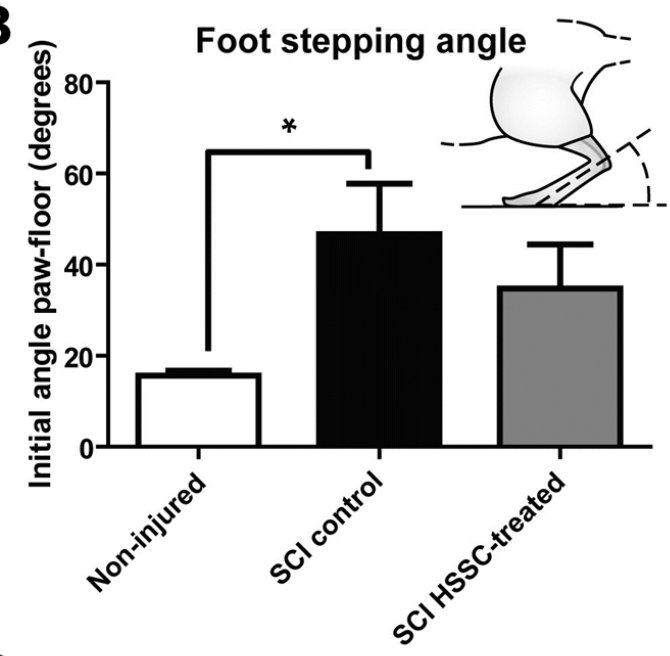

Relative LogMEP

(Baseline vs. Terminal)

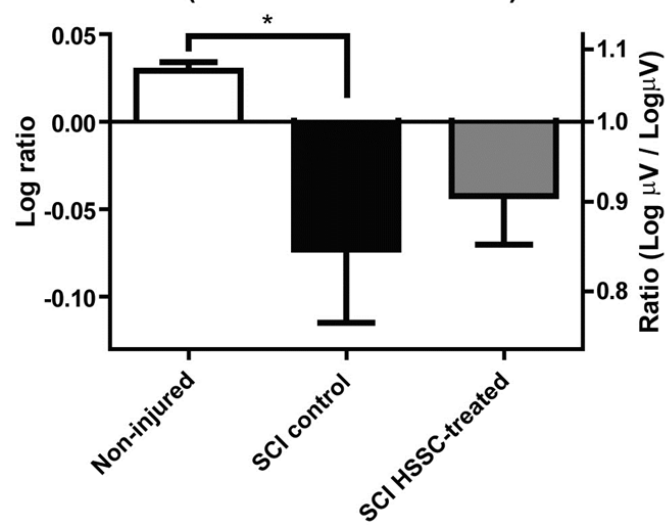

Figure VI-8: Effect of spinal HSSC grafting on locomotor function (BBB), foot stepping angle, ladder climbing test and motor evoked potentials. A: Weekly measurement of the BBB scores modified for the L3 injuries (left y-axis) and regular BBB scores (right $y$-axis) showed progressive recovery in both HSSC-grafted and control SCI animals. While there was a trend toward better motor performance in HSSC-grafted-animals, this effect was not significant for both scoring systems. B: Single Frame Analysis showed a tendency towards regaining normal foot stepping angles between the paw and floor (measured at stance-phase initiation; see insert/drawing in B) in SCI-HSSC-treated animals. However, the angles were not significantly improved if compared to $\mathrm{SCl}$ controls. C: Using the ladder climbing test, we found a significant decrease in number of correct steps in $\mathrm{SCl}$ animals if compared to naïve controls. No significant difference was seen between $\mathrm{SCl}$ control and SCI-HSSC-treated animals if analyzed at $\mathbf{2}$ months after treatments. D: Motor Evoked Potentials recorded at baseline (i.e., before injury) and at 8 weeks post injury showed a significant decrease only for the SCl-control animals. No significant difference between HSSC-grafted and control SCl animals was detected. 

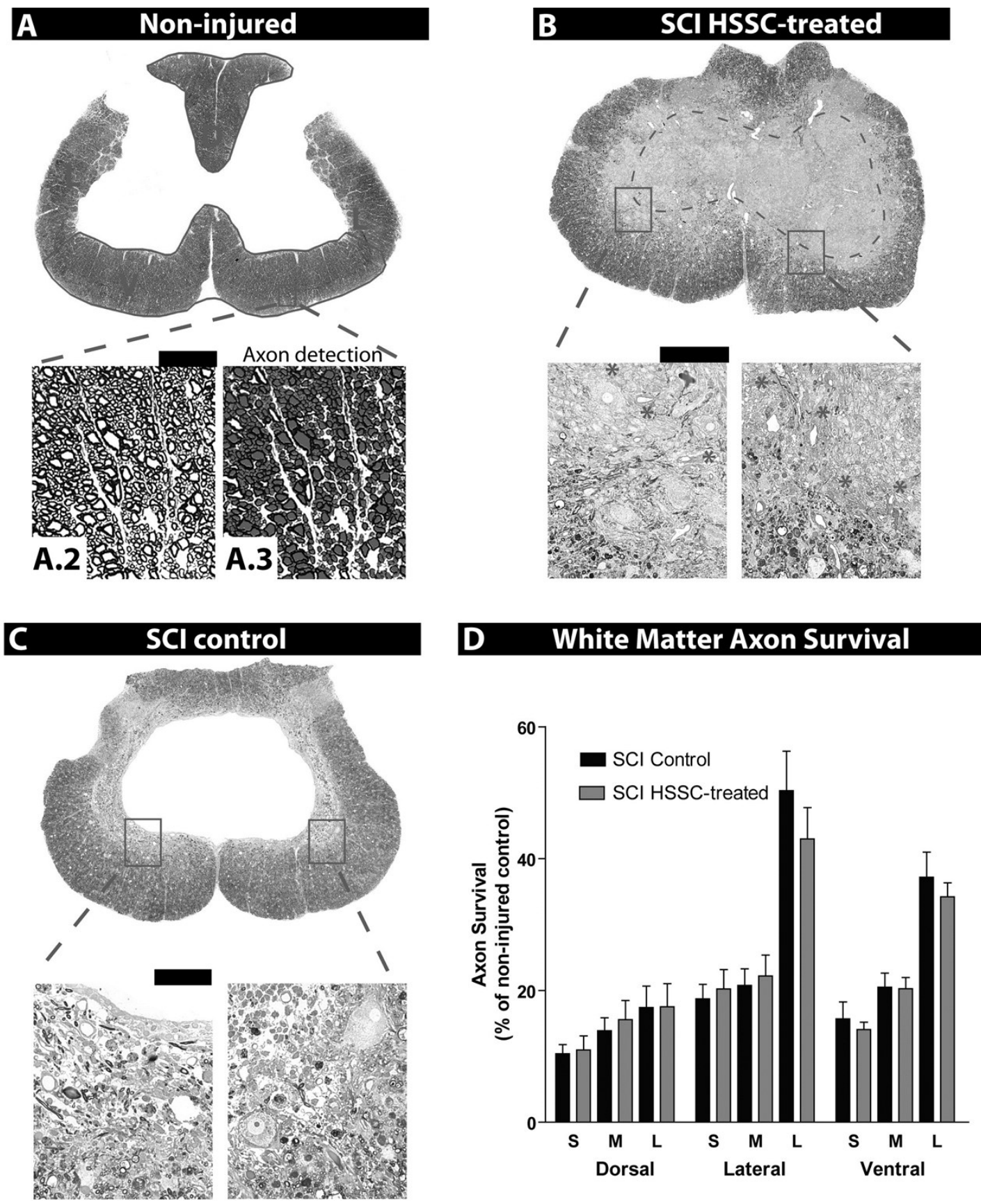

Figure VI-9: Axonal survival in the injury epicenter. A: Schematic diagram of the axon counting design used in our current study. Axons were counted in plastic osmium-stained sections in the dorsal, lateral and ventral funiculi using ImageJ software. An example of the detection threshold to identify individual axons in a selected field is shown in A2 and A3. B: Transverse plastic section depicting a bilaterally distributed graft (red dashed line) and completely filling the cavity created by previous spinal compression. Note that the fusion of the graft with the host tissue is so advanced that the border between the previous injury-evoked cavity and the graft is difficult to delineate (red asterisks). C: An example of transverse spinal cord section taken from an animal receiving media injection. An extensive cavity occupying near completely the region of previous gray matter can be seen. D: Quantification of axons in SCl-control and SCl-HSSC-treated animals showed no significant differences if analyzed in dorsal, lateral or ventral funiculi or if sub-divided into axons of different caliber ( $S=s m a l l=0.3-1 \mu \mathrm{m}$; $M=$ medium=1-2.5 $\mu \mathrm{m}$; L=large=2.5-10 $\mu \mathrm{m}$ ). (Scale Bars: A-C: $500 \mu \mathrm{m})$. 
Systematic quantification of the total number of myelinated axons counted bilaterally in a control naïve animal showed 281,352 axons (Fig. VI-9A). Thirty-three percent was represented by small caliber axons, $57 \%$ by medium caliber axons and $10 \%$ by large caliber axons. In SCl-control animals,

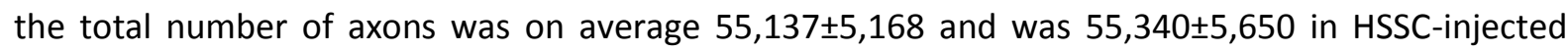
animals (Student's $t$-test: $P=0.98$; Fig. VI-9B-D). Intergroup statistical analysis of the axons at specific diameter (i.e., $0.3-1.0,1.0-2.5$, and $2.5-10 \mu \mathrm{m}$ ) showed $P$-values of $0.88,0.84$, and 0.51 (Student's $t$ tests) between SCl-control and SCI-HSSC-grafted animals, respectively. Intergroup statistical analysis of the axons at specific funiculi (i.e., dorsal, lateral, and ventral funiculi) showed $P$-values of 0.73 , 0.82 , and 0.72 (Student's $t$-tests) between SCI-control and SCI-HSSC-grafted animals, respectively (Fig. VI-9D). Additional intergroup analyses of axon survival categorized by both size and location/funiculus did not show significant differences between SCl-control and SCl-HSSC-grafted animals ( $P>0.36$; Student's $t$-tests) (Fig. VI-9D).

\section{Discussion}

In the present study, we investigated the treatment effect of spinally grafted GMP-grade human fetal spinal cord-derived neural stem cells (HSSC) in a L3 spinal compression injury (SCI) model in rats. HSSC were grafted into and around the epicenter of the contusion-injured L3 spinal segment at 3 days after spinal trauma in continuously immunosuppressed Sprague-Dawley rats. In comparison to control $\mathrm{SCl}$ animals with no treatment or receiving intraspinal injections of media only, the intraspinal grafting of HSSC led to a progressive and significant improvement in: 1) gait/paw placement, 2) muscle stretch-induced spasticity, and 3) mechanical and thermal sensitivity. These behavioral benefits were associated with robust graft survival and near complete injury-cavity-filling effect with grafted cells and corresponding lack of syringomyelia otherwise seen in control SCl cellnon-treated animals. In addition, the development of putative GABA-ergic synapses between grafted neurons and interneurons and/or $\alpha$-motoneurons of the host were identified. These data demonstrate that intraspinal grafting of HSSC into an injured spinal cord segment in the acute phase of injury represents a safe and effective treatment modality. This cell-replacement therapy was effective in providing qualitatively- and quantitatively-defined functional benefits and also led to significant and long-term improvement in the structural integrity of previously trauma-injured spinal cord segments. 


\section{Rationale for early spinal cell-replacement therapy after spinal trauma}

Both experimental and clinical data show that the spinal pathological processes following acute spinal injury are in part characterized by continuing axonal/neuronal degeneration, which can then continue for months to years after injury (Bramlett 2007, Ek 2010, Falci 1997, Hagg 2006, Wirth 2001). It is believed that such an ongoing axonal degeneration is in part the result of the lack of local trophic support associated with loss of neurons/glial cells at and around the injury epicenter. Thus, the use of treatment strategies which can replace or supplement the loss of the local neurotrophic activity and are initiated during this acute period should thus lead to a measurable treatment effect. Previous studies have demonstrated that neural stem cells of mouse, rat or human origin are a rich source of extracellularly released trophic factors (such as NGF, BDNF, GDNF, EGF, IGF-1, and VEGF) in in vitro cultured cells and that these cell populations retain a high level of neurotrophin expression after in vivo grafting in naïve animals and in a variety of neurodegenerative models including spinal injury and transgenic amyotrophic lateral sclerosis (ALS) models (Fagerlund 2011, Hawryluk 2012, Lu 2003, Xu 2006, Yan 2004, Yan 2007). In addition, using long-term post-grafting survival periods, it was shown that in vivo grafting of neural precursors with neurogenic potential into either the spinal cord at 9 days post spinal cord injury, the brain at 3 days post ischemic insult, or the CNS (brain or spinal cord) of adult or developing rats, there is the development of functionally and morphologically-defined synaptic contacts between grafted neurons and the neurons of the host (Cummings 2006, Lepore 2006, Rota Nodari 2010).

Based on these characteristics of neural precursors (NPCs), the use of NPCs for acute spinal cord grafting after trauma serves three purposes. First, it serves to provide local trophic support in the areas of previous injury (provided that grafted cells are able to home and survive long-term once grafted into the injured spinal cord milieu) and to minimize or halt the process of progressive axonal/neuronal degeneration. Second, it serves to provide a cavity-filling effect by replacing previously injured-degenerated necrotic tissue and thus prevent the long-term (or progressive) formation of rostro-caudal cavitations (i.e., syringomyelia) (Wirth 2001). Third, by the development of synaptic contact with the host axons/neurons above and below the injury level it can potentially lead to formation of a functional relay through the injury site. 


\section{Effect of spinal grafting of HSSC on the recovery of motor function and muscle spasticity}

In our current study, a combination of several motor performance tests were employed including open field modified BBB scoring, Catwalk gait analysis, inclined ladder climbing, single frame hind limb motion analysis, and myogenic motor evoked potentials to identify the degree of motor function recovery after cell grafting. The changes in muscle spasticity in lower extremities (i.e., below the level of injury) were also measured using a computer-controlled ankle rotational system (Marsala 2005). The catwalk gait analysis showed significantly improved paw placement in HSSCinjected $\mathrm{SCl}$ animals when compared to control $\mathrm{SCl}$ animals. In addition, a significant suppression of otherwise exacerbated muscle spasticity response measured during ankle rotation was seen in celltreated animals.

A previous study has shown that spinal grafting of adult rat spinal cord-derived NPCs at 7 days post thoracic contusion injury (MASCIS/NYU weight drop from $12.5 \mathrm{~mm}$ ) resulted in significant improvement in BBB/open field and grid-walk scores over 9 weeks after grafting in rats (Hofstetter 2005). Similarly, spinal grafting of mouse NPCs immediately after L2 spinal segment compression in mice resulted in a significant improvement in posture and hindlimb motor response to hindlimb extension with no significant improvement in the grid-walk or grip test (Boido 2011). Another study showed significant improvement in BBB-score over 8 weeks after spinal grafting of rat embryonic tissue-derived spinal cord-derived NPCs and Glia Restricted Precursors (GRPs) at 9 days after thoracic contusion injury (MASCIS/NYU weight drop from $25 \mathrm{~mm}$ ) in rats (Mitsui 2005). In the same study, no significant improvement in the grid-walk was seen.

Mouse embryonic stem cell-derived NPCs grafted spinally at 9 days after thoracic contusion injury (MASCIS/NYU weight drop from $25 \mathrm{~mm}$ ) were associated with a significant BBB-score improvement in rats measured up to a month after cell grafting (McDonald 1999). More recently, a significantly improved functional recovery (as assessed by BBB-scoring and spinal cord evoked potentials) after spinal grafting of acutely dissociated rat embryonic spinal cord tissue at 2 weeks post injury or after spinal injections of HSSC (the same cell line as used in our current study) at 1 week post injury was reported in a Th3 complete transection model in rats (Lu 2012). In the same study, to further facilitate the cell engraftment in the region of complete spinal cord transection, the cells were first embedded into the fibrin matrix enriched with a cocktail of neurotrophins and growth factors, which was then placed into a $2 \mathrm{~mm}$ long tissue gap created by the previous Th3 complete transection. Similarly, we have previously demonstrated that spinal grafting of HSSCs (same cell line as used in the current study) 21 days after induction of spinal ischemic injury in Sprague-Dawley rats resulted in a significant recovery of motor function and amelioration of spasticity when assessed up to 12 weeks 
post-grafting (Cizkova 2007). In another study, human fetal NPCs (cultured as neurospheres or enriched for oligodendrocyte precursors) grafted spinally at 7 days post thoracic contusion injury in rats (MASCIS/NYU weight drop from $25 \mathrm{~mm}$ ) showed a temporary significant improvement during the initial 5 weeks post cell grafting, but this effect was no longer present at longer (up to two months) post-treatment intervals (i.e., a faster recovery was seen in HSSC-treated animals) (Alexanian 2011).

Several other studies showed no motor improvement (as assessed by BBB or grid-walk) after spinal grafting of mice NPCs, or rat GRPs or GRP-derived astrocytes (GDAs, induced by CNTF in vitro pretreatment), when grafted 8 days after thoracic contusion injury in rats (MASCIS/NYU weight drop from $25 \mathrm{~mm}$ ) or immediately after cervical dorsolateral funiculus transection in rats, respectively (Davies 2006, Davies 2008, Macias 2006). However, immediate spinal grafting of GDAs, generated after Bone Morphogenetic Protein-4 (BMP-4) in vitro pre-treatment in a rat cervical dorsolateral funiculus transection model, did result in significantly less foot-faults on the grid-walk test (Davies 2006, Davies 2008). No motor improvement (as assessed by BBB-scoring) was seen after spinal grafting of human GRPs or BMP-4-induced GDAs at 9 days after thoracic contusion injury in athymic rats (MASCIS/NYU weight drop from $25 \mathrm{~mm}$ ) (Jin 2011).

These results indicate that cell replacement therapies which employ cell lines with both gliogenic and neurogenic potential can be beneficial in promoting motor/sensory recovery after $\mathrm{SCl}$. In addition, these studies and our current study demonstrate that a clear therapeutic effect can be achieved once cells are grafted during the early post-injury period (i.e., 3-7 days after spinal trauma).

\section{Effect of spinal grafting of HSSC on the recovery of sensory function}

In our study, we assessed the sensory function below the level of injury (hind paws) by measuring the mechanical and thermal thresholds for supraspinally mediated escape behavior. We found significant improvement of both $\mathrm{SCl}$-induced mechanical and thermal hypoesthesia. It is important to note that the sensory thresholds did not yet plateau at the end of the 2-month survival period. We speculate that an additional quantitative and qualitative improvement in the sensory function would likely be seen should a longer post-grafting interval be studied. In addition to sensory tests, quantitative analysis of spinal parenchymal markers indicative of developing (spinal) hypersensitivity (i.e., CGRP/GAP43, an indicator of aberrant sprouting of primary sensory neurons (Macias 2006, Ondarza 2003), and Iba1 staining, a marker of microglia activation (Hains 2006)) were studied and showed a significant decrease in CGRP staining intensities in HSSC-treated animals if compared to SCI controls. This suggests that the recovery/decrease in sensory thresholds observed in our study is not a result of aberrant sprouting or microglia activation. 
Consistent with the observations from our study, previous studies from other laboratories have demonstrated similar findings. Spinal grafting of human fetal neural cells (A2B5+NG2+) 7 days after thoracic contusion injury (MASCIS/NYU weight drop from $25 \mathrm{~mm}$ ) partially, but significantly, improved below-level thermal hypoesthesia at 7 weeks post-grafting (Alexanian 2011). Spinal grafting of adult rat spinal cord neurogenin-2-expressing cells at 7 days post rat thoracic contusion injury (MASCIS/NYU weight drop from $12.5 \mathrm{~mm}$ ) improved below-level hyposensitivity (assessed by fMRI) (Hofstetter 2005). Similarly, spinal grafting of human GDAs at 9 days after thoracic contusion injury in athymic rats (MASCIS/NYU weight drop from $25 \mathrm{~mm}$ ) resulted in a trend towards improvement of a SCl-induced increase of frontpaw (above-level) withdrawal thresholds to thermal stimuli (Jin 2011).

The comparison of the effect of spinal NPC grafting on sensory function (measured thresholds for escape behavior) used in our study with other studies reporting on ( $\mathrm{SCl}$ - or therapy-induced) decreased paw withdrawal thresholds is to some extent speculative, because the relation between paw withdrawal thresholds and supralesional escape behavior is still not well-defined (Baastrup 2010a). With that in mind, previous studies have demonstrated that GRPs or GRP-derived astrocytes (GDAs, acquired through CNTF treatment), which were spinally grafted in rats immediately after high cervical dorsolateral funiculus transection, resulted in decreased forepaw mechanical and thermal withdrawal thresholds (i.e., below the level of injury) (Davies 2008). GDA induction from GRPs with BMP-4 prevented this hypersensitivity and the related occurrence of aberrant CGRP sprouting in the cervical spinal dorsal horn (Macias 2006). Spinal grafting of human BMP-4-induced GDAs at 9 days after thoracic contusion injury in athymic rats (MASCIS/NYU weight drop from $25 \mathrm{~mm}$ ) resulted in significant improvements of a SCl-induced decrease of hindpaw (below-level) withdrawal thresholds to mechanical stimuli (Jin 2011).

Similarly, spinal grafting of human fetal NPCs 7 days post thoracic contusion injury (MASCIS/NYU weight drop from $25 \mathrm{~mm}$ ) resulted in a minor improvement of SCl-induced lowered below-level paw withdrawal reflexes, when grafted cells were A2B5+/NG2+ NPCs (not NCAM+/A2B5+ or $\mathrm{NCAM+/A2B5-)}$ (Alexanian 2011). Yet another study showed restoration of SCl-induced lowered tail withdrawal latencies to a heat stimulus as assessed up to 8 weeks after spinal grafting of rat embryonic spinal cord-derived NRPs and GRPs at 9 days after thoracic contusion injury in rats (MASCIS/NYU weight drop from $25 \mathrm{~mm}$ ) (Mitsui 2005). This indicates that a partial glial differentiation in grafted cells observed in the current study might be one of the mechanisms responsible for amelioration of aberrant CGRP sprouting and resulting decrease in spinal sensitization. 


\section{Differentiation of grafted cells and mechanism of HSSC-mediated therapeutic action}

In our current study, near pure population of nestin+ human fetal spinal stem cells were grafted intraspinally at 3 days after contusion-induced spinal cord injury. Analysis of the graft survival at 2 months after grafting showed a dense population of grafted hNUMA+ cells in grafted previously trauma-injured regions. In addition, numerous hNUMA+ cells which migrated out of the graft in distances ranging between 2-3 $\mathrm{mm}$ were also seen. Using human-specific antibodies against NSE and synaptophysin (markers of mature neurons), we have also shown that a majority of grafted cells developed into a neuronal phenotype. Many human specific synaptophysin+ boutons were found to reside in the vicinity of host neurons.

Quantitative analysis of the host axon survival in the injury epicenter showed no significant sparing effect in $\mathrm{HSSC}$-grafted $\mathrm{SCl}$ animals vs. medium-injected or untreated $\mathrm{SCl}$ animals. These data suggest that 1) the majority if not all axons which succumb to pathological processes resulting from secondary changes post injury such as edema, ischemia were already lost or irreversibly damaged at 3 days after trauma (i.e., the time point when the cells were grafted), or 2) regional cell grafting is not therapeutically effective in providing acute neuroprotection.

Analysis of the neurotransmitter phenotype in grafted cells showed the development of putative inhibitory GABA-ergic synapses with host neurons. These data show that the restoration of the local functional inhibitory circuitry by grafted cells can in part lead to the observed functional improvements. Similarly, in our previous study using the same cell line, we have demonstrated the development of putative GABA-ergic synaptic contacts between grafted neurons and persisting $\alpha$ motoneurons of the host in a rat spinal ischemia model. In the same animals, a significant amelioration of spasticity was measured (Cizkova 2007). In a recent study using EM analysis, we have confirmed the development of synaptic contacts with the host neurons at 9 months after intraspinal grafting of HSSC in normal non-injured immunodeficient rats (Hefferan 2012). Similarly, in a more recent study, the development of functional contacts and restoration of axon potential conductivity across the region of complete Th3 spinal transection by grafted HSSC was seen (Lu 2012).

In addition to restoration of the local motor circuitry, significant amelioration of otherwise increased spinal CGRP expression seen in non-treated $\mathrm{SCl}$ animals was measured in $\mathrm{SCl}$ animals receiving spinal injections of HSSC. Consistent with this observation, previous studies have demonstrated that improvement of local spinal GABA-ergic tone, as achieved by subcutaneous inoculation of a replication-incompetent herpes simplex virus (HSV) encoding GAD67 gene in a Th13 spinal cord hemisection model, led to a similar decrease in otherwise increased CGRP expression (Liu 2004). Second, previous studies have shown that spinally grafted HSSC show the expression of several 
trophic factors (GDNF, BDNF, and VEGF) at 2 months after grafting in SOD+ rats (Xu 2006). We speculate that the release of these tropic factors can potentiate the sprouting of persisting axons of the host below and above the injury and accelerate the development of new synaptic contacts particularly at longer post-grafting intervals.

Finally, we have demonstrated a near complete injury-cavity filling effect by the grafted cells at 2 months after grafting when the cells were grafted at 3 days after injury. This was in contrast to media-injected animals which showed consistent and extensive rostro-caudal spinal cord cavitation. These data suggest that early post injury cell grafting is desirable as it can effectively block the formation of the spinal cavity and its expansion and related long-term secondary spinal cord degeneration. A comparable cavity-filling effect and prevention in the progression of syringomyelia has been shown after spinal grafting of human embryonic or fetal SSCs in human patients with progressive post-traumatic syringomyelia (Biyani 1994, Falci 1997, Wirth 2001).

It is important to note that the cavity-filling effect demonstrated in our current study was achieved without the use of any supporting matrices or additional topical growth factor(s) delivery. In our preliminary study, we have determined that while the density of grafted cells is relatively low to fill the cavity-forming region, the grafted cells continue to proliferate after grafting to the point when a cavity is near completely filled with grafted cells [unpublished data]. The cell proliferation is inhibited once the cavity is filled and after that the cells differentiate normally. That the cells do not develop into pre-neoplastic or neoplastic cells has been assessed in a 9-month tumorigenicity study with nude rats whose Th9 spinal cord segment was first injured by contusion (manuscript in preparation). Similarly, using the same cell line as used in our current study, we have previously reported a comparable low level of mitotic activity in grafted cells at 6 weeks to 9 months after grafting in naïve immunodeficient rats or immunosuppressed minipigs (Usvald 2010).

In our current study, we demonstrate a functionally-defined treatment effect after spinal grafting of human GMP-grade fetal spinal stem cells in immunosuppressed SD rats with previous L3 compression injury. This treatment effect was expressed as a significant improvement in motor and sensory function (gait/paw placement, stretch-induced muscle spasticity, and, mechanical and thermal sensitivity). In addition, an effective filling of trauma-induced spinal cavity with grafted cells was seen in HSSC-treated animals at 2 months after grafting. Jointly, these data demonstrate that the use of this clinical grade NSI-566RSC cell line with already established favorable clinical safety profile represent a potential cell candidate for cell replacement therapy in patients with previous spinal traumatic injury. 
$\stackrel{\text { 옥 }}{\stackrel{1}{1}}$ 


\section{V01. Tumorigenesi̊s sẩety stundy of human ईetal neural precursor cells i̊n SCI rats}




\title{
Preclinical safety assessment of contemporary experimental stem cell therapies for clinical trial authorization; an instructive example with neural stem cells
}

Sebastiaan van Gorp; Osamu Kakinohana; Estevan Ruiz Limon; Marjolein Leerink; Elbert A. Joosten; Mickey Dugich-Djordjevic; Michael Hefferan; Karl Johe; Martin Marsala

\begin{abstract}
A main concern for any novel therapy for clinical applications is its safety. The same holds true for stem cell based therapies. In particular the potential tumorigenic property of many contemporary experimental stem cell therapies requires distinct preclinical safety assessments. As no standard guidelines or any stem cell safety studies have yet been published, it is still a matter of debate on how to perform such assessments. In this study we describe the preclinical assessment of the safety of a promising human fetal spinal cord derived neural stem cell therapy as part of a phase 1 clinical trial application. The design of the ensuing safety study is the result of a dialogue between researchers in this field, and the national drug authority (the Food and Drug Administration). This study and the concomitant efficacy/therapeutic studies resulted in the approval of the currently ongoing clinical trial in patients with amyotrophic lateral sclerosis. Therefore, to provide a reference and guide for future preclinical stem cell safety studies, we here report on the main considerations and outcomes of our preclinical safety study.
\end{abstract}




\section{Introduction}

An essential prerequisite of a successful transfer of any experimental therapy into clinical practice is a tolerable safety profile; i.e. its toxicity. Typically, a toxicity study is conducted after the initial proof of efficacy (disease modification) is established and follows a relatively well defined guideline of a supervising agency, like the Food and Drug Administration (FDA)-guidelines-based experimental design (Food and Drug Administration 1997). An Investigational New Drug application for clinical trials (IND) to the FDA requires study reports which assess potential toxicities in their target tissues of animal species that are relevant for the clinical development plan of the drug (U.S. National Archives and Records Administration 2012b).

Essentially, this is not different for cell-based therapies, but a key item of toxicity potentially associated with any stem/progenitor-cell-based therapy is tumorigenicity; i.e. the propensity of grafted donor cells to form tumors, either by itself or by inducing host cells. Either can occur in the targeted tissue, but also, due to cell migration, in non-targeted tissues. Generally, the risk of tumorigenesis is higher when allogeneic cell-based therapies have long-term, or even permanent, survival and integration, have differentiation and proliferation capacity, have a higher chance/need for a systemic route of administration, or are not irreversibly implantable.

Unfortunately, practical guidelines to assess tumorigenic potential of any group of IND candidate stem cell population in an animal model are not defined. One test of tumorigenicity is provided in an FDA guidance document (Food Drug Administration 1993). In this test, a bolus cell suspension consisting of at least $10^{\wedge} 7$ cells are injected subcutaneously in at least ten athymic (immunodeficient) mice and assessed up to a month. However, for development of a therapeutic cell-based product, an additional tumorigenicity study would be required in which the cells are injected into the clinically intended target tissue with a cell dose at the very least consistent with planned human dose. Obviously, although tumorigenesis is an important outcome when assessing the safety profile of a cell-based therapy, the other 'classical' signs of toxicity should not be neglected and require extensive health observations.

Here, we report on the safety assessment of NSI-566RSC, a human fetal spinal cord-derived neural stem cell population (Yan 2007), developed for spinal neurodegenerative diseases and spinal cord injury for final use in human trials. NSI-566RSCs are developed using reproducible and well-defined protocol which permit a long-term expansion of human spinal stem cells isolated from cervical spinal cord of human first trimester fetuses. NSI-566RSCs are now well-established and fully characterized in vitro (Guo 2010, Lunn 2010). The cells are produced under Good Manufacturing Practice (GMP) conditions (U.S. National Archives and Records Administration 2012a, U.S. National Archives and 
Records Administration 2012d). Efficacy studies to define the therapeutic use of this particular cell line after spinal grafting have been reported to i) extend life span and regional Alpha-motorneuron protection in SOD+ ${ }^{(693 A)}$ transgenic rats (Hefferan 2012, Xu 2006), ii) improve motor function and ameliorate spasticity in spinal ischemia-injured Sprague-Dawley (SD) rats (Cizkova 2007), iii) improve motor function after $2 \mathrm{~mm}$ long full spinal transection in SD rats (Lu 2012), and, iv) improve hind limb function, and mechanical and thermal-stimulus evoked sensory function, and no engraftment procedure related deterioration in L3 spinal cord contused SD rats [unpublished observation]. With the availability of a stable, safe to engraft, and effective product, all logical prerequisites for a preclinical safety assessment are met.

This safety study was designed in conjunction with FDA reviewers (Office of Gene and Cell Therapy/CBER/FDA) for guidance on how to design the safety study to include suitable tumorigenicity assessment. Following are the main considerations to assess the toxicity of (neural) stem cell-based therapy, with special focus on tumorigenesis, as a frame of reference.

As a first prerequisite, the study must be performed in a Good Laboratory Practice (GLP)-compliant manner (including external auditing) and in a contract research facility (U.S. National Archives and Records Administration 2012c). Any safety study is designed specifically to have a high sensitivity for potential systemic and local toxicities. The goal is to show the safety of a final stable product (e.g. GMP-compliant) in a concentration which is evidently higher than will be used in human patients. Formulating such a maximal tolerated dose also involves anticipating the normal proliferation of the cell-based therapy. In addition, factors possibly enhancing the inherent proliferating potential (or tumorigenicity) of the grafted cells in/on the targeted tissue should be taken into account (e.g. spinal cord disease increased mitotic potential of NSI-566RSCs). Preliminary dose finding studies are thus advisable. Next, standard toxicology endpoints are studied, including mortality, clinical observations, and (histo-)pathology of targeted and non-targeted tissues, with a special focus on tumorigenesis through examination of all suspected lesions and masses by an external certified pathologist. The use of athymic ( $\mathrm{RNU}^{-} / \mathrm{RNU}^{-}$) animals is first choice since they possess genetic immunodeficiency (no T-cells), which is required for perfect cell survival while the rats remain relatively resistant to simple/opportunistic infections due to an intact innate immune system. In agreement with FDA reviewers, this study needed to hold at least a final 30 treated animals and 30 appropriate control animals with a survival of 6 months, with periodic toxicity assessments, to be considered adequately conclusive.

In conclusion, the goal of the present study is to characterize the safety profile of NSI-566RSCs after spinal grafting in spinal cord contused athymic rats, as defined by i) toxicity, specifically entailing ii) 
tumorigenesis, and iii) any additional health observations. In addition, although strictly not part of a toxicity assessment, it was decided best to collect additional supporting safety data by characterizing NSI-566RSC after long-term and high dose spinal cell grafting in terms of iv) safety for neurological function (motor and sensory), and v) its acceptance by/integration in host tissue.

The data from this study and the abovementioned efficacy and safety studies were used for IND application and successfully led to IND approval of NSI-566RSC. NSI-566RSC is currently in ongoing Phase I clinical trials in amyotrophic lateral sclerosis patients (Glass 2012, Neuralstem Inc. - Emory University 2000).

\section{Animals, materials, and methods}

The study design and animal usage were reviewed and approved by the Institutional Animal Care and Use Committee. Animal welfare for this study was in compliance with the U.S. Department of Agriculture's (USDA) Animal Welfare Act (CFR 9 - Parts 1, 2, and 3) (Department of Agriculture's Code of Federal Regulations 9 2009), and the Guide for the Care and Use of Laboratory Animals (National Research Council 1996).

\section{Animals}

Rats, Athymic Nude (rnu-/rnu-) - Hsd: RH-Foxn1rnu (Harlan, Madison, WI, USA) were used. A total of 90 male rats were enrolled, of which 8 were used for long descending anterograde tract labeling (Fig. VII-1). Animal ages at injury ranged from 10 to 13 weeks, and body weights varied between 309 to 345 grams.

\section{Animal housing}

Animals were single-housed in cages with irradiated bedding. Acclimation times prior to the experiment varied, but were at least 8 days. Environmental controls were set to maintain temperatures from 640 to $79^{\circ} \mathrm{F}$ ( $18^{\circ}$ to 26 으), with fluorescent lighting on a 12 hour/12 hour on/off inverted cycle. Animals were fed irradiated Harlan Teklad Global 18\% Protein Rodent Diet T.2918.15 (Harlan Laboratories, Placentia, CA) ad libitum. In addition, animals that were selected for addition behavioral testing (see below) also received Fruit Crunchies $^{\mathrm{TM}}$ (BioServ product \# S05798-1) and HydroGel $^{\mathrm{TM}}$ (ClearH2O ${ }^{\circledR}, \mathrm{ME}$ 04101). Animals were provided with purified water (Mountain Dairy, Riverside, CA, USA) ad libitum. 


\section{Injury Surgery}

The day prior to surgeries the back of the animals was shaved. On SCl surgery days, each animal received lactated ringer's ( $5 \mathrm{~mL}$, s.c.). Animals were anesthetized with isoflurane (5\% for induction, $\sim 2 \%$ for maintenance, in air). The surgery site was wiped with alcohol and chlorhexidine diacetate solution (2-4\%). Rat body temperature was maintained by a water blanket heating system. The skin over the vertebral column was opened, paravertebral muscles were dissected away, and the animal was mounted onto a Stereotaxic frame (Stoelting Lab Standard Stereotaxic - Single, Cat\# 51600 Lab Standard) with Spine Adaptors (Stoelting, Cat\# 51695 Rat Spinal Adaptor). A thoracic spinal segment (Th10) was then exposed using a dental drill. A moderate injury was made by letting the rod of a MASCIS (NYU) Impactor dropped from a height of $12.5 \mathrm{~mm}$ onto the exposed surface of the spinal cord. Next, the impactor was removed immediately, the animal was detached from the frame, the surgical site was irrigated with sterile saline, layers were closed with absorbable Vicryl suture, and Bacitracin/Neomycin/Polymyxin (triple antibiotic) ointment was applied to the incision site. Cefazolin $(10 \mathrm{mg} / \mathrm{kg}$, s.c.) was also given at the day of surgery.

\section{Post-surgical Care}

All animals received Sulfamethoxazole and Trimethoprim oral suspension, (USP $200 \mathrm{mg} / 40 \mathrm{mg}$ per 5 $\mathrm{mL}$ ) in the water ( $5 \mathrm{~mL}$ per $250 \mathrm{~mL}$ ) for $1-2$ days before, up to 30 days after initial contusion, and as needed in case of infections. Animals were checked for bladder retention at least two times daily for the duration of the study. If full, bladders were emptied by manual expression. Consecutively, the surgical area was checked. Ketoprofen $(4.0 \mathrm{mg} / \mathrm{kg}$, s.c.) was given approximately one hour prior to surgery and approximately every 24 hours for up to 48 hours. Animals found ill were given up to 5 $\mathrm{mL}$ (s.c., $\leq 2$ daily) Lactated Ringer's solution until improvement was observed. Hexa-Caine Spray and/or Bacitracin/Neomycin/Polymyxin triple antibiotic ointment was used to treat scabs or wounds.

\section{Cells}

The cells, named "NSI-566RSC", were produced by Neuralstem Inc. (Rockville, MD, USA), as described before (Hefferan 2011). The grafted cells were a clinical lot (Lot No. CRL471925-3, passage 12), a human neural stem cell line derived from fetal spinal cord of 8 gestational weeks. They were suspended in hibernation buffer at $10^{\wedge} 4$ cells/ $\mu \mathrm{L}$. Each batch (and vehicle) was prepared at Neuralstem Inc (Rockville, MD, USA) one day before surgery and shipped by overnight service in temperature monitored containers. 


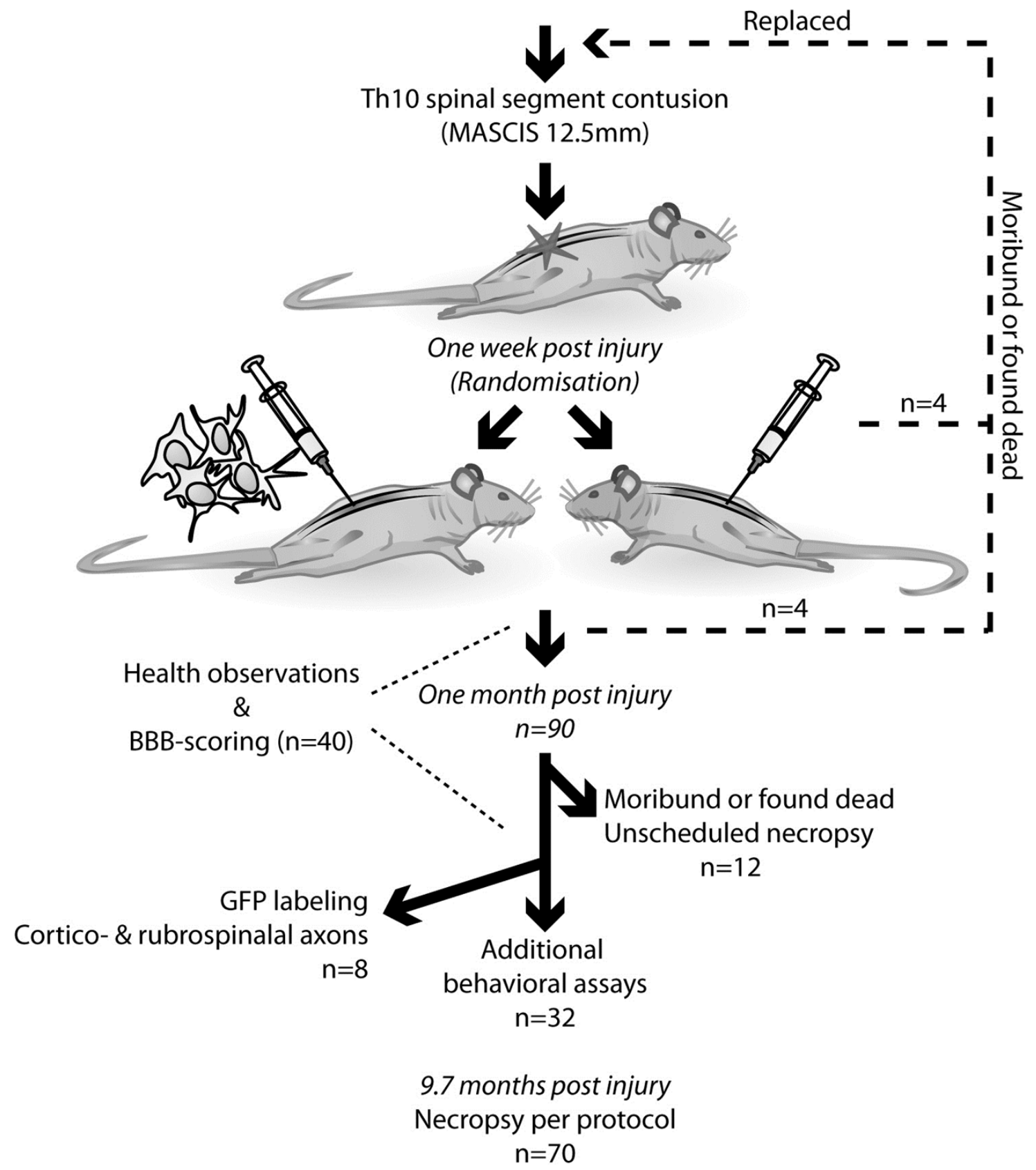

Figure VII-1: Study outline. Animals received a low thoracic moderate spinal cord injury with a MASCIC/NYU impactor (10 grams dropped from $12.5 \mathrm{~mm}$ high). They randomly received either vehicle or NSI-566RSCs at one week post-injury through multiple intraspinal/-parenchymal injections. All health observations and behavioral assays we performed while blinded for treatment. Eight animals were found moribund or dead before one month post-grafting and had been replaced. Twelve animals were found moribund or dead after the one month post-grafting timepoint and underwent unscheduled necropsy. Remaining animals survived for nearly ten months after grafting. 


\section{Dosing surgery}

One week following $\mathrm{SCl}$ surgeries, prior to grafting, animals were allocated to groups by stratified randomization based on body weights. Animals were assigned to either the vehicle group $(n=45)$ or the cell-graft group $(n=45)$. There were no exclusion criteria for dosing surgery other than appearing healthy enough. Moribund animals or animals found dead were replaced (up to 30 days post injury).

The day prior to grafting the animals were anesthetized to remove any remaining skin sutures and to shave the back of the animals. At the beginning of a grafting day (7 or 8 days after SCI), NSI-566RSC's viability was determined. The spinal cord was re-exposed. The dorsal aspect of the vertebra immediately caudal to the existing laminectomy was removed using the dental drill. Special care was taken to keep the dura intact. For the current safety assessment, a maximum feasible cell dose was estimated by several preliminary dose-range finding studies using subcutaneous and intraspinal delivery. The cell suspension (Group C) or vehicle/hibernation buffer-only (Group V) was injected as follows:

1) 20 injections peripheral from the injury epicenter ( $1 \mu \mathrm{L}$ each; $2 \mu \mathrm{L} / \mathrm{min})$ were made bilaterally into about 2 segments above and/or below the injury epicenter at $1 \mathrm{~mm}$ intervals.

2) 5 injections ( $5 \mu \mathrm{L}$ each; $2 \mu \mathrm{L} / \mathrm{min}$ ) were injected around the borders of injury epicenter. Hence, 25 injections were made in each spinal cord, resulting in a total of $45 \mu \mathrm{L}$ (cells suspended at $10^{\wedge} 4$ cells $/ \mu \mathrm{L}$, resulting in a total of $\sim 4.5 \times 10^{\wedge} 5$ cells).

The required volume of dosing for completing all injections of one animal was drawn into a $100 \mu \mathrm{L}$ Nanofil syringe (World Precision Instruments) with a 33-gauge needle (World Precision Instruments, Cat\# NF33BV). The syringe was mounted onto a manually controlled syringe holder/injector (David Kopf, Model 5000+5001) attached to the stereotaxic frame. The needle tip was lowered to a depth of 1-1.5 mm from the pial surface at the Dorsal Root Entry Zone (DREZ) into the spinal parenchyma, and the injection was made. After a thirty second pause, the needle was gently drawn out of the spinal cord. The syringe/needle ensemble was cleaned by repeatedly rinsing with and immersion in $70 \%$ isopropanol/water solution for a minimum of 15 minutes before and after each animal.

\section{Post injury Health observations, Measurements, and Specimens}

General Health Observations (GHOs) were performed on the dosing day, pre-dose, post-dose, and weekly thereafter. GHOs included weight, general appearance, stool appearance, toxicity symptoms and other appearing additional health issues (Table 1). In addition, tumor presence was assessed by full body palpation. Any palpable cell mass was measured using a caliper. All animals were observed daily to ensure no loss of animals for necropsy. 
All behavioral testing was performed under red light or dark conditions.

\section{Open field locomotor rating}

Locomotor recovery after spinal cord contusion injury was monitored using the Basso, Beattie, and Bresnahan (BBB) open field locomotor rating scale (Basso 1995). In the present study, the BBB score was obtained weekly until eight weeks post-injury, biweekly up until week 20, and every four weeks thereafter. Each examination was conducted by two experienced examiners and during five minutes. The animals selected for BBB testing comprised of the last cohorts of 20 animals in both therapeutic groups ( $\mathrm{n}=40$ total).

Additional behavioral testing was conducted on animals shwing weight support during the last BBB assessments. Behavioral testing was conducted approximately 1.5 months prior to sacrifice. For training/testing, animals were transferred to the testing room in their original housing and allowed to habituate to the room for 30 minutes, during which white noise was generated.

\section{Activity assessment in an open field}

One week prior to the beginning of the test trials, animals were adapted to the apparatus/arenas (76 $\times 152 \times 50 \mathrm{~cm} ; \mathrm{W} \times \mathrm{L} \times \mathrm{H})$ for five minutes on each day. After habituation, the animals were placed in the center of a clean arena and tracked by a video tracking system under dark conditions (night/active portion of their diurnal rhythm), over a period of three hours. The distance traveled and a number of various movement variables was assessed (EthoVision, Noldus Technology, The Netherlands) (Noldus 2001). Three separate observation periods were evaluated. Each observation period was separated by at least 24 hours. During testing, animals had access to Hydrogel and food pellets.

\section{Beam walk}

Rats were trained to traverse elevated $(1 \mathrm{~m})$ narrow beams $(3,2.5$, and $2 \times 200$ inches; $\mathrm{W} \times \mathrm{L})$ towards a darkened goal box $(10 \times 10 \times 10$ inches). Only motor impaired animals would produce foot faults (slips from beam), which number than represent a motor deficit. Bright light and white noise were produced near the start to promote beam crossing. Rats were allowed to fall from the beam (onto a container with padding). For training, each rat was placed in goal box for two minutes and afterwards placed on the balance beam for 1 minute per trial. The rat was considered trained when it was able to remain on the beam for three consecutive trials. Mean scores out of two trials for each 
beam were used (i.e. right and left hind paw foot faults, and falls). The animal was allowed to take $\sim 5$ min to complete a crossing. In between runs an animal was placed in their home cage for $\geq 15 \mathrm{sec}$. If an animal was able to cross a beam for at least two thirds, it was also tested on the narrower beam (i.e. 2.5 or 2 inch).

\section{Catwalk Gait Analysis}

The CatWalk apparatus (CatWalk 7.1, Noldus Technology, The Netherlands) was used to quantify gait parameters by footprint analysis during walkway crossings (Hamers 2006). Animals walk down a horizontal glass walkway $(109 \times 15 \times 0.6 \mathrm{~cm} ; \mathrm{L} \times \mathrm{W} \times \mathrm{H})$, of which the glass is illuminated along the long edge. The light only enters the (side of the) glass and reflects merely internally (when the glass is bordered by air). As an animal crosses the walkway, light reflects off of the animal's paws, producing a series of bright footprints when viewed through the glass, which are recorded by a video camera. Hence, testing was performed in a darkened room. Walkway crossing was stimulated by rewarding the animal with little Fruit Crunchies $^{\mathrm{T}}$, placed at the end of the walkway. In conjunction, animals were further motivated by food restriction prior to training/testing. Hence, food was removed from their cages approximately 18 hours before testing (and returned after training/testing). In addition, the following criteria concerning walkway crossings needed to be met: (1) the animal needed to walk uninterrupted across the walkway, at a constant pace, and (2) a minimum of three such crossings per animal were required. Data from three proper crossings was averaged for statistical analysis.

\section{Sensory function assessment (mechanical and thermal)}

In this test, pain thresholds for a supraspinal response (vocalization or escape behavior) to a belowlevel evoked mechanical or thermal stimulus were assessed. The animals were habituated to the setup, test room, and investigator for one week (no responses were elicited), twice daily for five minutes, prior to testing. The investigator held the animal in an upright position, fixed the tested hindpaw, and then the hindpaw was stimulated. When a response was elicited, the stimulus was stopped immediately and the maximal pressure elicited was recorded. Both hind paws were tested for four times while alternating between paws, with at least a 1 hour interval between trials. The first scores of each paw were removed.

The mechanical stimulus was created using a rigid tip mounted on a pressure transducer (IITC life sciences Electronic von Frey Anesthesiometer, Cat\# 2393, Woodland Hills, CA), which was operated by a second investigator (SVG). The stimulus was applied on the dorsal side of a hind paw. 
Compressing the paw of the animal with the tip on the pressure transducer at the distal metatarsal or metacarpal area in a gradual incremental fashion (paw rests on table surface).

For eliciting the thermal stimulus an infrared beam apparatus (Ugo Basile, Cat\# 37360, Collegeville, PA, USA) was used. For testing, the investigator held the animal's plantar side of a hind paw over the infrared light beam mounted in the apparatus.

\section{Necropsy}

The post-grafting survival period varied between $272-274$ days ( 10 months). Animals were sacrificed by $2 \mathrm{mg}$ pentobarbital and $0.25 \mathrm{mg}$ phenytoin $(0.5 \mathrm{~mL}$ of Beuthanasia-D, Intervet/ScheringPlough Animal Health Corp., Union, NJ, USA) followed by transcardial perfusion of saline, trailed by $4 \%$ paraformaldehyde (phosphate buffered). The central nervous system was dissected out and preserved in $4 \%$ paraformaldehyde. The following tissues were also taken out and placed in $10 \%$ formalin:

Skeletal muscle (thigh), skin, adrenals, aorta, rectum, cecum, colon, duodenum, esophagus, epididymides, eyes with optic nerves, femur, Harderian gland, heart, ileum, jejunum, stomach, kidneys, spleen, liver, lung with bronchi, mesenteric lymph node, mammary gland, pancreas, thymus, thyroid with parathyroid glands, trachea, pituitary, prostate, mandibular salivary glands, tongue, sciatic nerve, testes, seminal vesicles, and bladder.

Animals found clinically ill and moribund were euthanized, per protocol. On these animals, and all animals found dead, a gross necropsy was conducted (i.e. external surfaces and orifices, musculoskeletal system, cranial cavity (and brain), neck with associated tissues, and thoracic, abdominal and pelvic cavities (incl. associated organs and tissues).

At necropsy, any tissue with discoloration, lesion, necrosis, distension, malformation, and/or suspected tumor/mass were sent for external histopathological evaluations by a board-certified veterinary pathologist (J.E. Sagartz DVM PhD DACVP, Seventh Wave Laboratories LLC, Chesterfield, $\mathrm{MO})$, as were the histological sections of the central nervous system (see below). Evaluation was performed in a manner blinded to treatment nature. Severity grades for pathology diagnoses were based on a 4 point scale as follows: Grade1= minimal, Grade $2=$ moderate, Grade 3 = marked and Grade $4=$ severe. After completion of pathology analysis, the pathologist was unblinded and the final conclusion was made. 


\section{Central nervous system histology}

Each cord was photographed against a ruler $(\mathrm{cm})$ along with its identification number (Fig. VII-2). The cords were bisected into approximately equal rostral and caudal pieces and embedded horizontally into a gelatin block $(6.5 \times 4.5 \times 2.5 \mathrm{~cm} \mathrm{L \times W \times H})$. Spinal cords from $16-20$ animals were embedded in 4 horizontal layers in a block 1 (Fig. VII- 8). The rostral pieces were placed in the first and third layers and the caudal pieces were in the second and fourth layers, with the ventral side facing down. The layers were separated by $\pm 0.4 \mathrm{~cm}$ of gelatine. The cords (columns) were separated by $\pm 0.2 \mathrm{~cm}$ from each other. After solidification of the gelatin block, it was placed in $30 \%$ sucrose in $4 \%$ formaldehyde for 48 hours. Then, it was frozen in cold 2-methylbutane using dry ice, cryostat sectioned horizontally ( $40 \mu \mathrm{m}$ thick), and mounted with intervals of 12 sections. Whole brains were also embedded into gelatin blocks, cryostat sectioned coronally at $40 \mu \mathrm{m}$ thickness. All stained slides were sent to a Board-certified veterinary pathologist for evaluation (J.E. Sagartz DVM PhD DACVP, Seventh Wave Laboratories LLC, Chesterfield, MO). For histopathological stainings every $24^{\text {th }}$ section was used.

The spinal cords used to assess the neuronal integration (see below) were not gelatin embedded, but were frozen directly (after $48 \mathrm{~h}$ in $30 \%$ sucrose) using isopentane $\left(-80^{\circ} \mathrm{C}\right)$, mounted in OCT compound (Tissue-Tek ${ }^{\oplus}$ ) and cryostat sectioned at $30 \mu \mathrm{m}$ in the horizontal, sagittal, or transverse plane, and collected for free-floating immunofluorescence staining.

Stainings used entailed: hematoxylin \& eosin (H\&E), human neurofilament antibody (h.HO14; rat 1:100; human specific axonal marker; gift from Dr. Virginia Lee, University of Pennsylvania, PA, USA), Glial Fibrillary Acidic Protein antibody (h.GFAP; rabbit 1:500; human specific astroglial marker; Origene, Rockville, MD, USA ), and Green Fluorescent Protein antibody (GFP; chicken 1:2000; Aves, Tigard, OR, USA), Neuron-Specific Enolase antibody (h.NSE; mouse 1:500; human specific neurite marker; Vector Labs, Burlingame, CA, USA) Synaptophysin antibody (h.Syn; mouse 1:500; human specific synaptic marker; Millipore, Billerica, MA, USA), Human Nucleus antibody (h.HuMA; mouse 1:200; Millipore, Billerica, MA, USA), Glial Fibrillary Acidic Protein antibody (GFAP; mouse 1:500; Sigma-Aldrich; St. Louis, MO, USA), Oligodendrocyte lineage transcription factor 2 antibody (Olig2; rabbit 1:1000; Abcam ab81093; Cambridge, MA, USA), Ki67 antibody (proliferation marker; Abcam ab16667; 1:100; rabbit), and DAPI (In Prolong ${ }^{\circledR}$; Life Technologies, Carlsbad, CA, USA). Immunostainings were finished using diamino benzidine (DAB) staining or with fluorescentconjugated secondary donkey antibodies (Alexa ${ }^{\circledR}$ Fluor 488 \& 647; Jackson Immuno Research, West Grove, PA, USA; \& Alexa ${ }^{\circledR}$ Fluor 555; Invitrogen; 1:500). The Ki67 immunostaining was also counterstained with $0.1 \%$ Neutral Red and finished with DAB. 


\section{Evaluation of histological integration of grafted cells}

A small group of $\mathrm{SCl}$ animals ( $\mathrm{n}=8 ; 4$ with cell treatment), was used only to assess neuronal integration. Injury and treatment was identical up to almost two months prior to perfusion. Then animals underwent general anesthesia during which they received four brain injections of recombinant Adeno-Associated Virus (AAV) engineered to express Green Fluorescent Protein under the human synaptophysin promoter (AAV.synGFP; neuronal specific expression) into both motor cortices and both nuclei rubri. Stereotaxic coordinates used for the injections were: motor cortex: bregma $-2.0 \mathrm{~mm}$, lateral $3.0 \mathrm{~mm}$, depth $1.5 \mathrm{~mm}$, red nucleus: bregma -5.8 , lateral $0.8 \mathrm{~mm}$, depth 7.1 $\mathrm{mm}$. Each injection consisted of $5 \mu \mathrm{L}$ of the virus suspension at $10^{\wedge} 13$ particles $/ \mathrm{mL}$ and was injected over 5 min period using $32 \mathrm{G}$ stainless steel needle. The purpose of the virus injections was to label the host corticospinal and rubrospinal (motor) fibers in the spinal cord.

\section{Statistical analysis}

Results were analyzed using ANOVA (one-way, or two-way group $x$ time repeated measures, and using a fixed-effect model), with a Bonferroni post hoc test for multiple comparisons. If the unequal variances were observed (Bartlett's test), the Kruskal-Wallis test with Dunn's post hoc comparisons were used. Results are expressed as means with the standard deviations of the mean (SD), unless specified otherwise. To analyze differences between the two groups, we used student's t-tests or Mann-Whitney test (Non parametric), or a repeated measures ANOVA (when appropriate). All statistical analyses were performed two-tailed, and a P-value of 0.05 was considered significant. Between-group variations are reported as cell-injected vs. vehicle-injected.

\section{Results}

In this study 41 animals were injected with NSI-566RSCs and 41 received vehicle injections, of which 4 had been replaced before dosing and 4 within the first month after dosing, with equal occurrence among both groups (Fig. VII-1). Eight additional animals were used for descending tract labeling (vehicle: $n=4, N S I-566 R S C s: n=4)$. A total of twelve (six animals from each group) out of all 82 animals were found ill, moribund, or dead during the course of the study and underwent unscheduled necropsy. The remaining 78 animals underwent scheduled necropsy. Cell viability was recorded to exceed $70 \%$ prior to all grafting procedures. 


\section{External pathology report of targeted tissue}

The external report on Central Nervous System histopathology was made by a board-certified veterinary pathologist (J.E. Sagartz DVM PhD DACVP, Seventh Wave Laboratories LLC, Chesterfield, $\mathrm{MO}$ ) and based on observations made in sections of all 82 animals (without labeled descending motor tracts), for each staining.

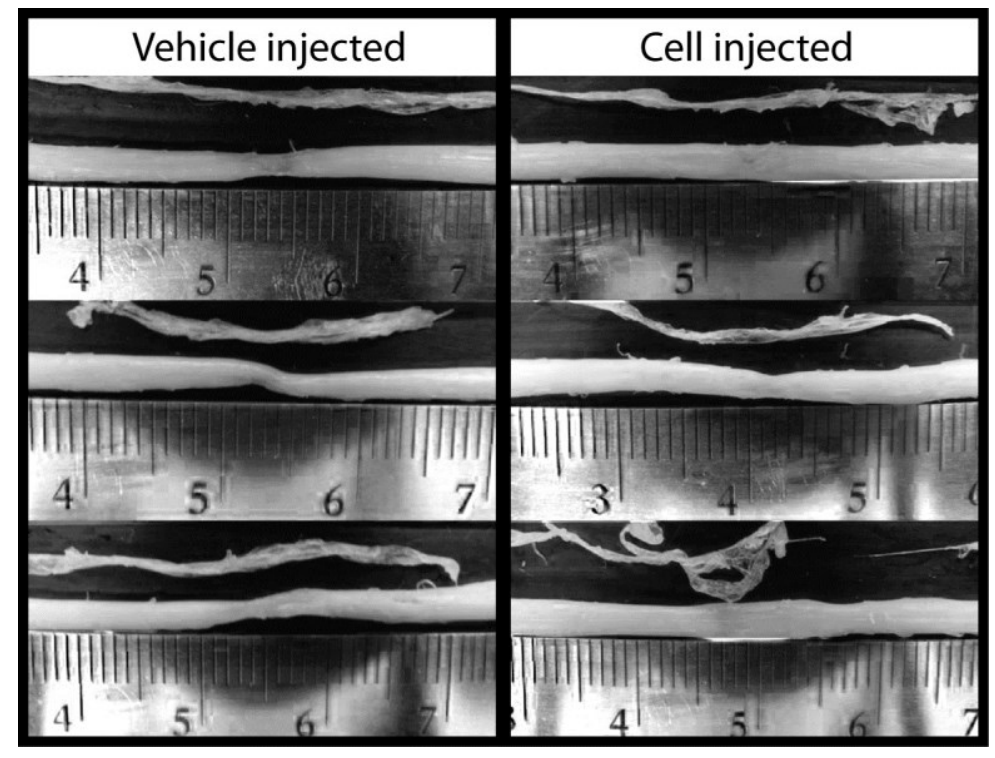

Figure VII-2: Representative examples of the gross-morphology of vehicle- and NSI-566RSC-injected spinal cords after a 10 month in vivo period. Note the difference in volume-loss at the lesion site between vehicle- and NSI-566RSC-injected spinal cords.
Macroscopically, no tumors were noted. Traumatic tissue destruction (i.e. loss of actual spinal cord volume) that graded mild to severe was recorded in the anterior caudal spinal cord segment (lesion site) of $32 / 40$ vehicle control animals and 11/41 NSI-566RSC injected animals. Tissue transparency, as an indication of subacute degeneration, was observed in gray or white matter at the injury/injection site of $5 / 40$ vehicle controls and 30/41 test rats (Fig. VII-2). The severity of

the degeneration was classified as mild to moderate, being primarily moderate in the majority of affected rats from both NSI-566RSC-engrafted (90\%) and vehicle-injected (80\%) animals.

Microscopically, grafted NSI-566RSCs were generally present in a dispersed, random pattern with no recognizable organization. Occasionally, discrete foci of h.NuMA immunoreactive cell clusters were observed which were present at or near injection sites as well as in the central canal at and adjacent to the lesion site. The amount of Ki67 immunostained (mitotic) cells at the lesion site of vehicle control rats was always at low levels, whereas in the remaining spinal cord no Ki67 immunoreactive proliferating cells were visible. Of the NSI-566RSC-injected animals 52.5\% had low levels of Ki67 immunoreactive proliferating cells ( 0 to 5 cells/200 $\times$ field), $15 \%$ showed $5-20,25 \%$ showed $20-40$, and only $7.5 \%$ showed more than 40 cells per $200 \times$ field. Cell proliferation in these spinal cords was classified as orderly. 
Ninety-five percent (39/41) of the NSI-566RSC grafted rats showed an abundance of h.NuMA immunoreactive cells at the site of injury/injections, mostly filling the cavities/syringes entirely. Two animals showed low numbers of grafted cells in their spinal cords. While the majority of grafted (h.NuMA immunoreactive, human nuclei) cells were found both anterior and posterior in the caudal cord segments, a few grafted cells were also observed in the rostral cervical segment, the segment furthest from the injury/injection site.

Both NSI-566RSC-engrafted and vehicle-injected animals showed spinal cord lesion sites with increases in cellularity (cell density), based on H\&E stained sections, however, more so in grafted animals. The latter was deemed primarily due to the abundant presence of grafted, h.NuMA immunoreactive, cells. The increase in cellularity in vehicle injected animals was mainly due to the presence of GFAP immunoreactive astrocytes, probably related to the development of the astroglial scar.

There were no h.NuMA immunoreactive cells present in the brains of rats. And there were no intervention-related lesions in the brains of either vehicle injected or cell grafted rats. Hence, in conclusion, no major (histo-)pathological changes noted after NSI-566RSC spinal engraftment in spinal cord injured nude rats at almost 10 months.

Full body necropsy and external histopathology report of NSI-566RSC-engraftment-related tumor formation

Each animal underwent full body necropsy after sacrifice or when found dead. Any lesions/masses were isolated and offered for external histopathological analysis (as above).

Fourteen animals of the vehicle group and five of the NSI-566RSC grafted group showed lesions and/or suspected masses (i.e. swollen, discolored, inflamed, and/or shrunk organs/tissue). All of which were examined by the external pathologist. Two tumors were identified. Both tumors occurred in animals from the cell injected group:

\section{Body weights}

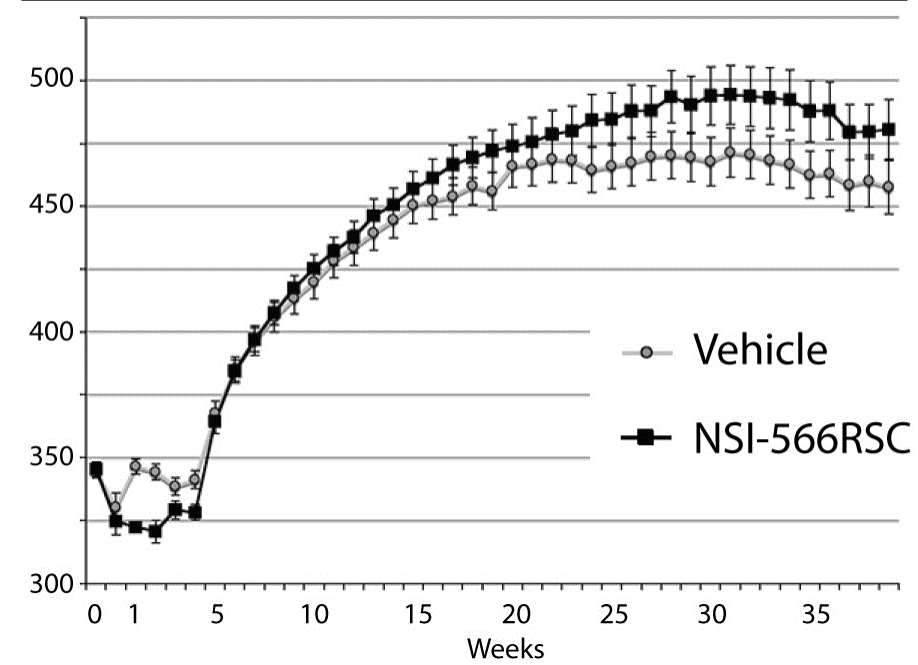

Figure VII-3: Body weights of athymic rats at baseline and during the course of the study (vehicle vs. NSI-566RSC treated). No significant difference in bodyweights was noted. Note that NSI-566RSC-engrafted animals tended to have slightly higher bodyweights. 
One had a trichoepithelioma of the skin of the left hind leg (a benign tumor with differentiation toward hair follicle epithelium).

The other had a multicentric malignant histiocytic sarcoma affecting multiple non-neuronal organs (abdominal and thoracic); a tumor commonly observed in aging rats.

All other lesions were also judged unrelated to NSI-566RSC engraftment.

In conclusion, no NSI-566RSC-engraftment-related tumors were identified.

\section{NSI-566RSC-engraftment-related toxicity}

Signs of toxicity were scored throughout the study in both the NSI-566RSC-engrafted and vehicleinjected group (Table VII-1). Symptom or toxicity ratings (number of symptoms) between NSI566RSC-grafted and vehicle-injected groups showed no differences ( $z=-.69$, Mann-Whitney U: $P=.49$ ). A staggered gait was the most frequent observation, which was observed in all vehicle and cellengrafted animals after th.SCCl. Comparisons of general appearance ratings (based on the number of different symptoms expressed) between both experimental groups revealed no differences between groups ( $z=-.90$, Mann-Whitney $U: P=.37$ ). Also stool appearance ratings (based on the number of different characteristics expressed) showed no differences between groups ( $z=-1.02$, Mann-Whitney U: $\mathrm{P}=0.31$ ).

\section{Additional in vivo health observations}

In addition to toxicity-related items, all changes in health status were monitored for both NSI566RSC-engrafted animals and vehicle-injected animals throughout the study. Final weights were $478 \pm 65$ grams for the cell-injected animals and $458 \pm 59$ grams for the vehicle injected animals (Fig. VII-3; Two Way ANOVA: F1,68=1.114, P=.30).

The most frequent clinical observation noted was scratching of the abdomen, which often also resulted in scabbed wounds. This was observed in the cell-injected group ( $n=24 / 41)$ as well as the vehicle-injected group ( $n=20 / 41$; Pearson's $\chi^{2}: P=.38$ ). In vivo palpable masses were noted in three animals in the vehicle group and six animals in the NSI-566RSC-engrafted group. No differences were seen between groups in speed of symptom onset (measured in weeks; $z=-.44$, Mann-Whitney U: $P=.66)$. Also, no differences were seen between both experimental groups when frequency of tumor expression was compared (Pearson's $\chi^{2}: P=.48$ ). Also, no differences in duration or frequency of manually bladder voiding requirements were present between groups. 


\begin{tabular}{|c|c|c|}
\hline Frequency of clinical observations & Vehicle & Cells \\
\hline GENERAL APPEARANCE OBSERVATIONS & & 96.2 \\
\hline Appears normal; bright, alert, and responsive & 37 & 37 \\
\hline Appears mildly depressed; quiet, slow to respond to stimulus & 1 & 1 \\
\hline Appears markedly depressed; lethargic, non-responsive & 3 & 4 \\
\hline Appears moribund; recumbent, unable to respond to stimulus & 1 & 2 \\
\hline Found dead & 1 & 1 \\
\hline STOOL APPEARANCE & & 96.2 \\
\hline Stool normal & 41 & 39 \\
\hline Stool soft & 0 & 2 \\
\hline Diarrhea, no blood or mucus & 0 & 0 \\
\hline Diarrhea with blood or mucus & 0 & 0 \\
\hline SYMPTOMS OF TOXICITY & & 96.2 \\
\hline No symptoms & 41 & 40 \\
\hline Mechanical repetition of movements (e.g., circling) & 0 & 0 \\
\hline Excessive grooming & 0 & 0 \\
\hline Self biting & 1 & 1 \\
\hline Excessive restlessness and irritability & 0 & 0 \\
\hline Lethargy & 4 & 6 \\
\hline Piloerection & 0 & 0 \\
\hline Difficulty breathing & 0 & 1 \\
\hline Tremors, twitches, and/or spasms & 1 & 1 \\
\hline Staggering gait & 41 & 41 \\
\hline Prostrate; unable to stand or walk & 1 & 2 \\
\hline Convulsions & 0 & 0 \\
\hline Death & 1 & 1 \\
\hline OTHER HEALTH FINDINGS & & 96.2 \\
\hline Tumor/mass palpable & 3 & 6 \\
\hline Scratching & 24 & 20 \\
\hline Swollen paw(s) & 8 & 6 \\
\hline Unscheduled Necropsy & 6 & 6 \\
\hline
\end{tabular}

Table VII-1: Main health observations after $\mathrm{SCl}$ in athymic/ $\mathrm{RNU}^{-1-}$ rats throughout the course of the study (i.e. 10 months). Data shows no differences in frequency of any health observation between treatment groups (vehicle- vs. NSI-566RS-injected; $z=-.69$, Mann-Whitney U: $P=.49$ ).

The following events were found at least once (slightly different events omitted for readability):

Tumor palpable, scratch/scab/sore, dehydration, lactated ringer given, cold to touch, favors a limb, bandage wrap applied, bloody/red urine, Crede maneuver, limb(s) dragging, eye discoloration, hunched, lacrimation, redness, swelling, hardness, pus(-tules), Cefazolin given, tooth discoloration, highly defensive, bent tail, tail tip discoloration, and missing tail tip

In summary, no differences in frequencies of toxicity symptoms and/or health status were noted between the NSI-566RSC-engrafted group and vehicle-injected group 
To assess the safety of NSI-566RSC on neurological function, behavioral assessments of neurological function were made.

\section{Locomotor function}

The Basso, Beattie and Bresnahan (BBB) locomotor scoring results are shown in figure VII-4A. Using the BBB scores locomotor function was evaluated up to nine months after th.SCCI. No significant differences in BBB scores were seen between NSI-566RSC-engrafted and vehicle-injected animals for BBB scores (Repeated Measures ANOVA: F135=.61, P=.44).

Sixteen animals of both the NSI-566RSC-engrafted and vehicle-injected group (32 in total) which showed weight support during BBB testing at the end of the trial, were subsequently used for additional behavioral sensory and motor assessments (see below). The additional behavioral sensory and motor assessments are no main outcomes for the safety study and were performed to provide supportive safety data. As such, these tests were performed in a fashion not strictly compliant with Good Laboratory Practice guidelines, for financial reasons.

\section{Sensory function}

At the end of the study, animals were tested for below-level pain thresholds for mechanical and thermal stimuli using both the right and left hind paws. Using escape-response thresholds for mechanical stimuli, no differences were seen between both experimental groups and tested paws (Fig. VII-4B; Two way ANOVAs: $\mathrm{P} \geq .31$; with $\mathrm{F} 1,35=1.06$ and $\mathrm{F} 1,35=.61$, respectively). Also, no differences were seen in the escape-response latencies for thermal stimuli between NSI-566RSCengrafted and vehicle-injected animals or between left and right hindpaws (Fig. VII-4C; Two way ANOVAs: $\mathrm{P} \geq .052$; with $\mathrm{F} 1,35=4.03$ and $\mathrm{F} 1,35=.13$, respectively). The effect size of the trend towards between-group difference for thermal stimuli was small (partial $\eta^{2}=.10$ ).

\section{Activity assessment}

Also at the end of the study, the activity of the animals was evaluated over several trials in an open field (three hours each; one to three trials per animal). Analyses were conducted to compare differences across a number of motor activity variables between cell-grafted and vehicle-injected animals. No significant differences were noted for total distance moved ( $P=.98$; Fig. VII-4D), frequency of mobility ( $P=.46$; Fig. VII-4E), total duration of movement ( $P=.07$; Fig. VII-4F), and mean velocity $(\mathrm{P}=.54)$. 

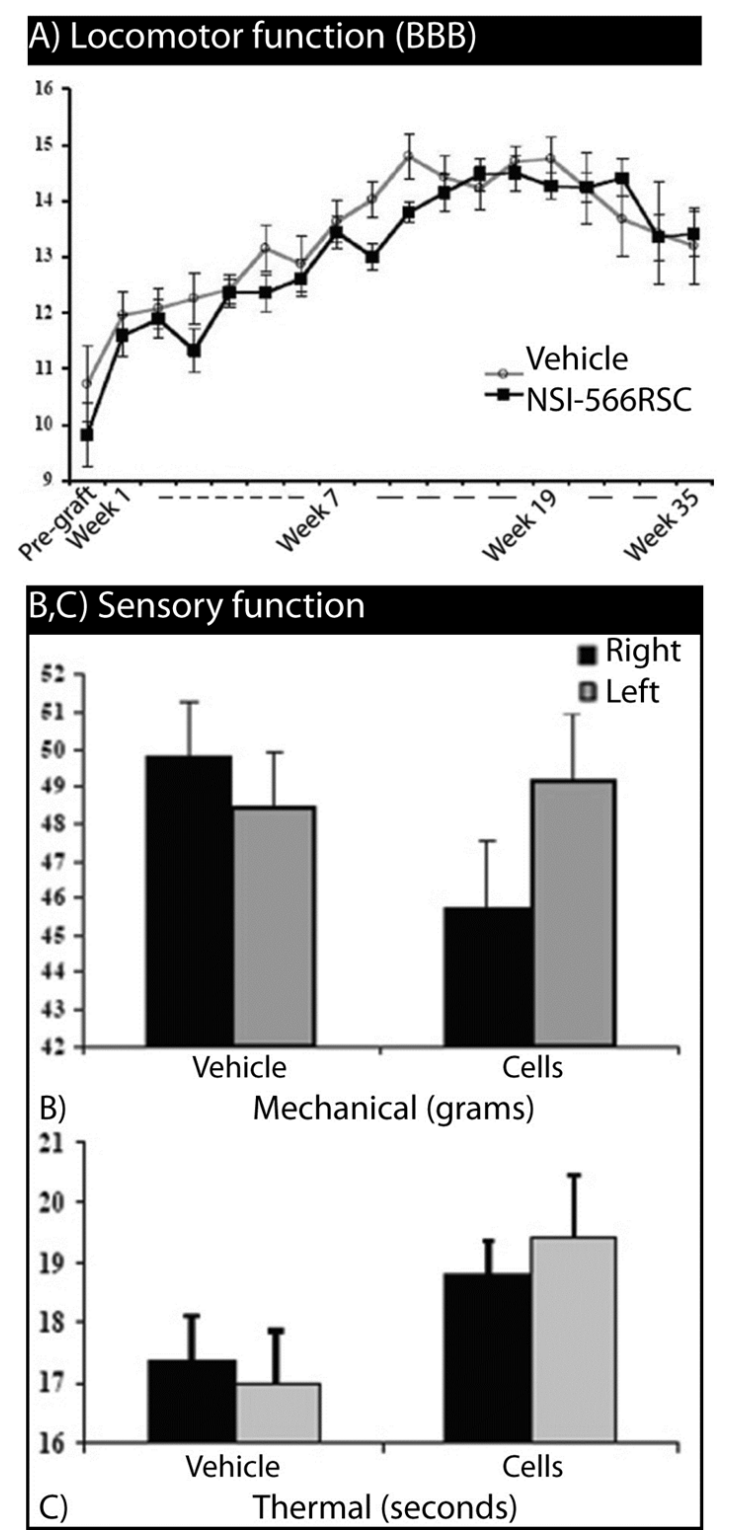

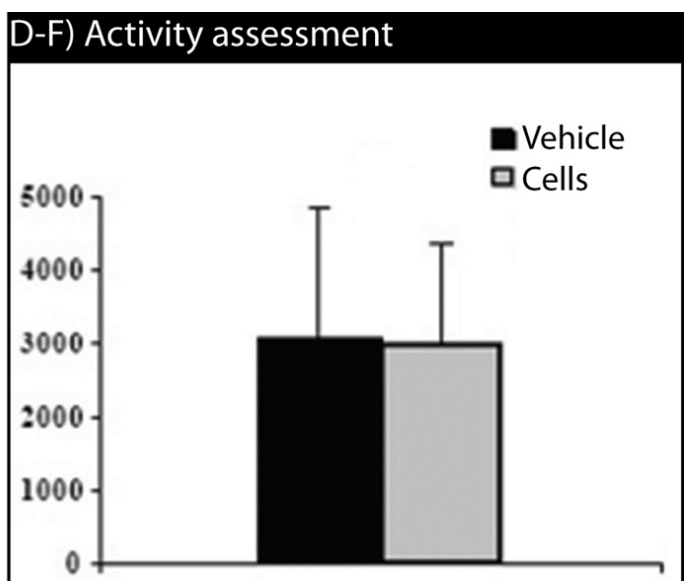

D)
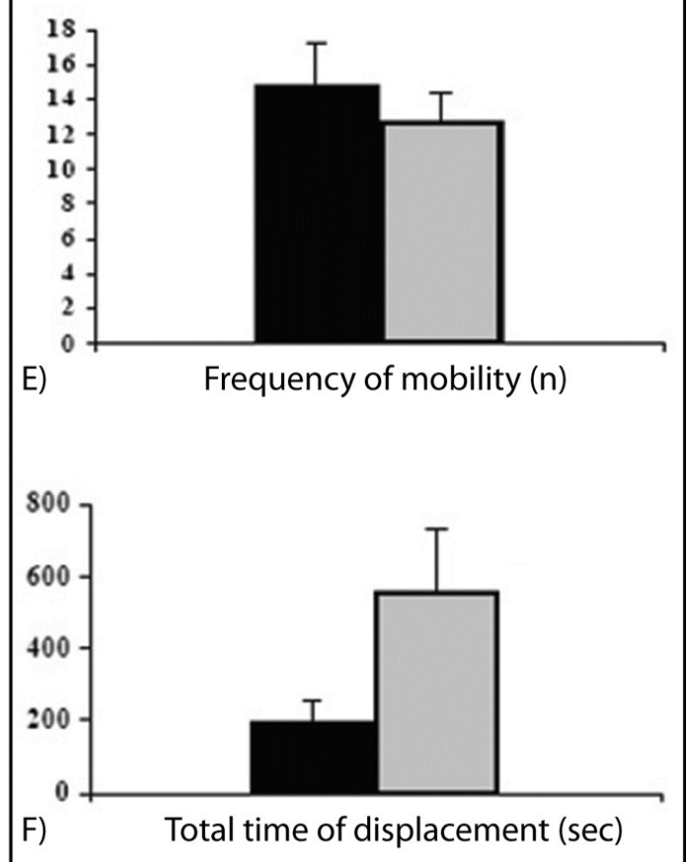

Figure VII-4: Behavioral results show no functional differences after SCI between groups (vehicle- vs. NSI566RSC-injected). A) Locomotor (BBB) scores of both treatment groups during the entire course of the study. A score of $\mathbf{0}$ was given if there was no spontaneous movement, and a score of 21 indicates normal locomotion. BBB scores generally increasing with time until week 17 post-graft, then dropping slightly until week 35 post-graft. No significant differences were seen between groups for BBB scores. B-C) Near the end of the study (8-9 months post-grafting), thirty-two of the animals were tested for thermal and mechanical pain thresholds. No differences in mechanical thresholds were seen (Figure B) between groups or between tested paws. Also, no differences in thermal sensory function were seen (Figure C) between groups or between tested paws. Note that while the treatment effect appears to be approaching significance, the effect size was small (partial $\left.\eta^{2}<.10\right)$. D-F) Also near the end of the study (8-9 months post grafting) the latter animals were tested for motor activity. No differences were noted for total distance moved (total distance traveled by the subject's center; Figure D), frequency of mobility (total number of samples involving any movement of the subject's center-point; Figure E), and duration of movement (total amount of time involving movement; Figure 2F). Means \pm SEMs. 


\section{Gait assessment by Catwalk}

Thirty out of the thirty-two animals were successfully trained (run duration: $2.5 \pm 0.2 \mathrm{sec} ; 2$ animals did not perform smooth complete runs and were excluded) and were recorded on the Catwalk at the end of the study (Fig. VII-5A-C). Cell-grafted $(n=14)$ and vehicle-injected $(n=16)$ animals were compared to investigate the effects of engraftment on different aspects of gait using the CatWalk method for gait-analysis. Eleven variables were selected for between group (NSI-566RSC vs. vehicle injected) comparisons and showed no significant differences for run duration $(P=.55)$, regularity index ( $P=.56$; Fig. VII-5A), hindpaw stride length ( $P=.99$; Fig. VII-5B), hindpaw Base of Support $(\mathrm{P}=.88$;

Fig. VII-5C), hindpaw stance duration $(P=.20)$, and hindpaw swing duration $(P=.85)$.

\section{Sensorimotor/Beam walk function}

Animals were tested sensorimotor integration based on their ability to traverse a beam (3", $2.5^{\prime \prime}$, or 2 " wide) without foot-faults. There were no statistical differences (Two way ANOVAs) between the NSI-566RSC and vehicle injected groups for number of falls $(F 1,12=.18, P=.68)$, left slips $(F 1,12=.03$, $\mathrm{P}=.87)$, and right slips $(\mathrm{F} 1,12=1.21, \mathrm{P}=.29)$; as seen in figure VII-5D-F, respectively.

Therefore, neither the BBB-locomotor test, sensory assessment, gait analysis, or sensorimotor/beam walk test showed functional differences between NSI-566RSC-engrafted and vehicle-injected animals. In summary, no decline in neurological function was noted after high dose spinal NSI566RSC engraftment in spinal cord injured nude rats for up to $\sim 10$ months. 

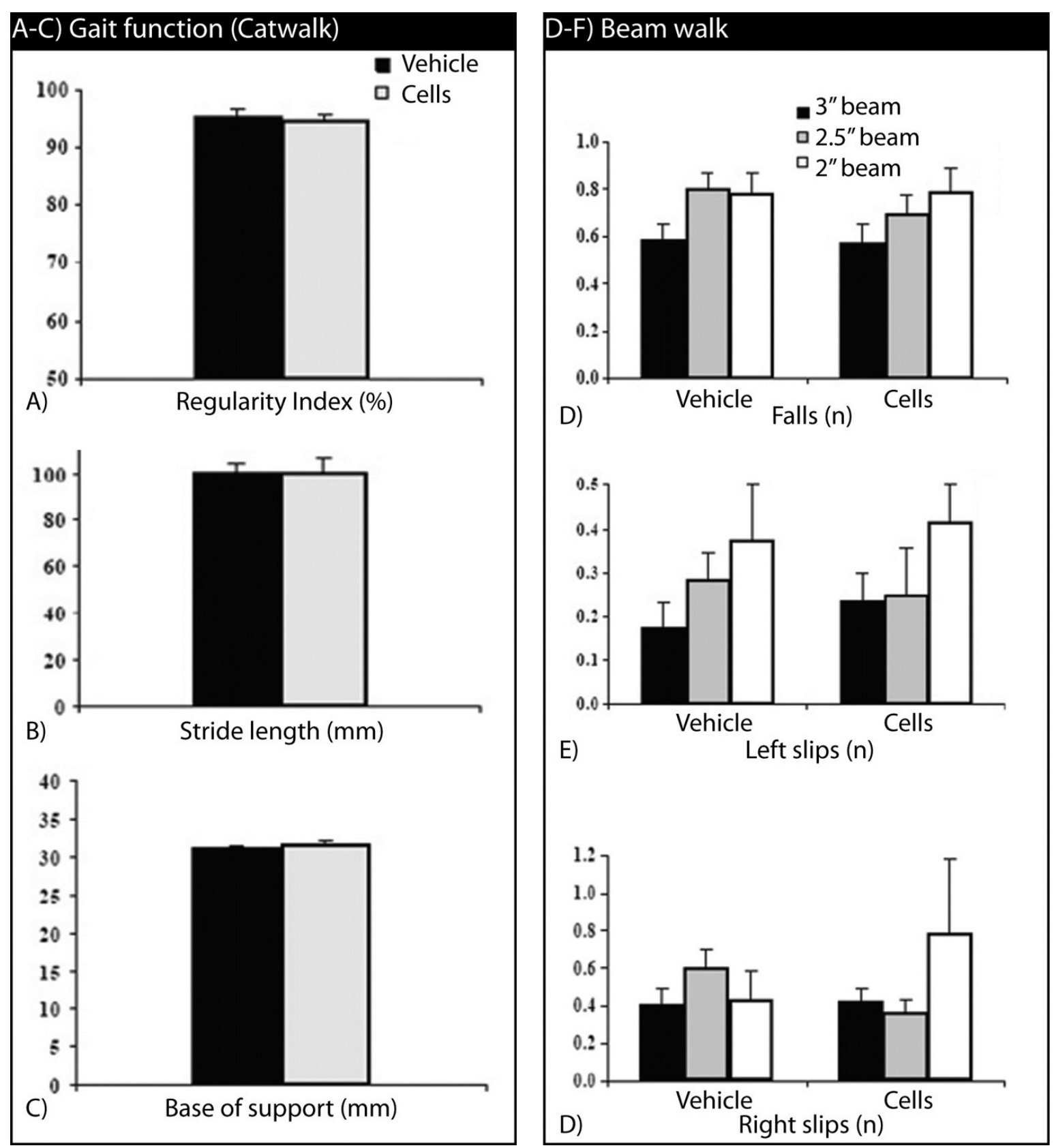

Figure VII-5: Behavioral results show no functional differences at 8-9 months after SCI between treatment groups (vehicle vs. NSI-566RSC treated; initiated one week post injury). A-C) Thirty out of thirty-two animals (16 of the vehicle treated animals, 14 of the treated animals) successfully completed the training and were recorded on the Catwalk for analysis of gait performance. Of special relevance in $\mathrm{SCl}$ are: regularity index (Figure A), hind paw stride length (Figure B), hind paw Base Of Support (mm, Figure C). There were no significant differences between treatment groups. D-F) Thirty-two animals were tested on the beam walk. All animals received two separate trials on the first (and widest, 3") beam. If the animals were not able to traverse at least two thirds of the distance across the beam, they were not tested on the next (narrower, 2.5" or 2") size beam. There was no statistical difference in falls or paw slips between the cell and vehicle injected groups. Means \pm SEMs.

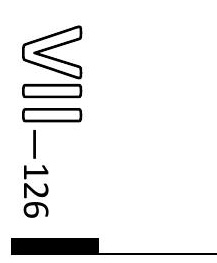




\section{Histological integration of grafted cells}

To analyse integration/compatibility with host tissue and neural differentiation of NSI-566RSCs, further immunostainings were performed.

Seventy-eight percent (32/41) of the NSI-566RSC-engrafted rats showed human-specific axonal neurofilament protein (h.HO14) immunoreactive axonal fibers (Fig. VII-6A) that projected laterally and orthogonally near the injury site (Fig. VII-6B), and both rostrally and caudally away from the contusion site (Fig. VII-6C). Also, at the lesion site, h.HO14 immunoreactive cells were found with better delineated and thicker axons (Fig. VII-6B), when compared to those at places more distant from the lesion site (Fig. VII-6C), therefore appearing to be in a more mature state. Nine NSI566RSC-grafted animals did not have adequate spinal cord sections for extensive evaluation, but h.HO14 immunoreactive cells were nonetheless observed in the cord. The average length of the h.HO14+ axons from five representative cords was $7.4 \mathrm{~cm}$ (SD \pm .32 ), covering $\pm 67 \%$ of the entire cord studied $(\sim 11 \mathrm{~cm})$. Hence, neuronal differentiation and neurite outgrowth show good compatibility of NSI-566RSCs with host tissue.

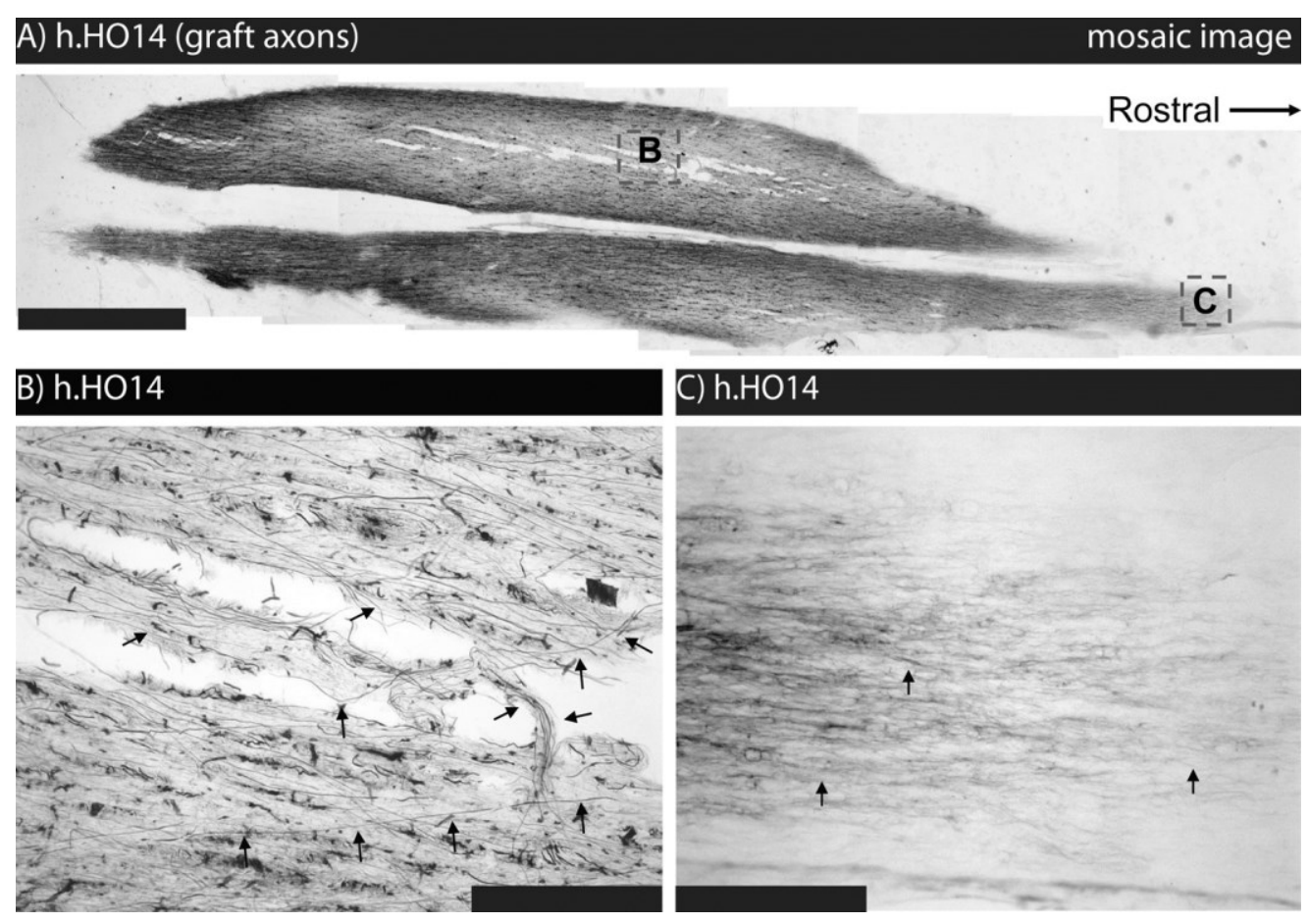

Figure VII-6: A human axon (h.HO14) staining of a nearly ten months in vivo surviving cell graft in an injured spinal cord from a athymic rat, showing graft survival, migration, and neuronal differentiation. A) Spinal cord injury epicenter and grafting site overview obtained from a mosaic montage of $2.5 \times$ images. B) Lateral and orthogonal spreads of bundles of human stem cells. Arrows depict HO14 bundles. Note the thick diameter of h.HO14+ fibers. C) The most rostral end of human stem cell fibers is shown. Arrows depict HO14+ fiber endings. Note the thinner diameter and rostro-caudal direction of the axonal endings, and compare to (more matured) axons in the contusion epicenter (B). Scale bar: $1 \mathrm{~mm}(A), 150 \mu \mathrm{m}(B, C)$. 


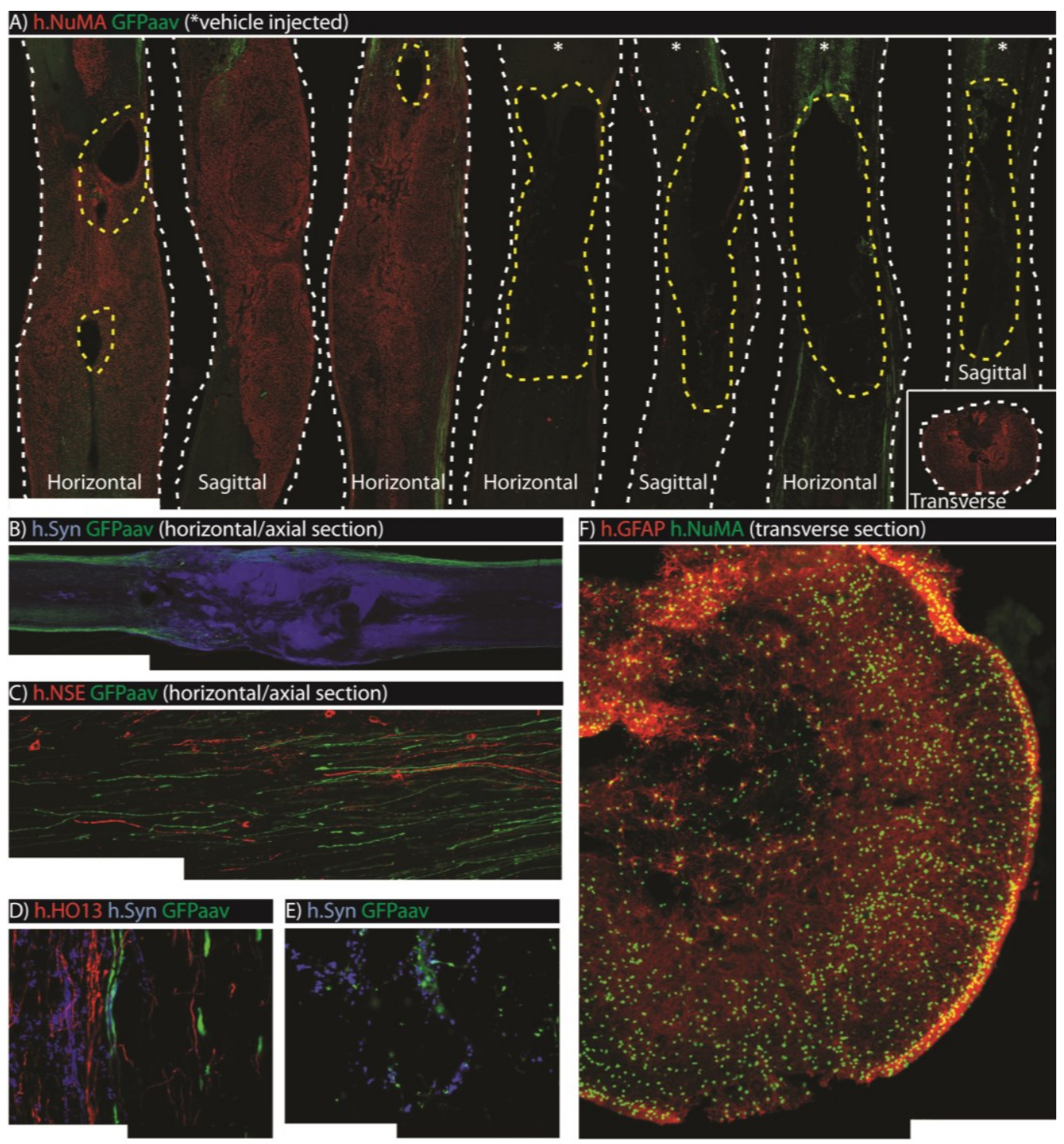

Figure VII-7: Immunostained spinal cords from spinal cord injured athymic rats, showing host GFP labeled descending motor tracts and graft survival, migration, and neural differentiation. A) Mosaic montages of GFP labeled host descending motor tracts (rubro- and corticospinal tracts; in green) and human nuclei (in red), showing widespread graft survival after $\sim 10$ months in the four animals with engrafted NSI-566RSCs. Note that host motor tracts traverse the graft. B) Human specific synaptophysin, indicating neuronal differentiation and synapse formations by NSI-566RSCs throughout the engrafted area, including areas of surviving host tissue. C) Human neurons stained by anti-Neuron Specific Enolase (in red), showing neuronal differentiation of the grafted NSI-566RSCs and formation of neurites, in relatively intact host tissue (i.e. white matter). D, E) Confocal images showing host GFP labeled descending motor tracts (in green) and human synapses stained with human specific anti-synaptophysin (in blue) and human axons (HO14; in red: panel D), indicating synapse formation between host and graft cells. F) Human specific GFAP (astrocytic marker; in red) expression in a transverse section of a spinal cord of a cell engrafted animal near the injury epicenter, indicating widespread astrocytic maturation of the NSI-566RSCs. Stained human nuclei (h.NuMA; in green) colocalizes with the human GFAP staining, showing human specificity of this GFAP staining. Scale bars: $4 \mathrm{~mm}(A, B), 200 \mu \mathrm{m}(C), 50 \mu \mathrm{m}$ (D, E), $0.5 \mathrm{~mm}$ (F) 
Next to the 82 animals of the main study, eight additional animals had their long descending motor axons labeled (four received NSI-566RSCs, four received vehicle only; Fig. VII-1). This was done to show integration of grafted NSI-566RSC in the host tissue on a more detailed fashion. These animals were also used to further characterize the neural differentiation of the NSI-566RSCs nearly 10 months after grafting.

NSI-566RSCs were again generally found to be located in a dispersed, random pattern with no recognizable organization and almost entirely filling up the injury cavities (h.NuMA/human nuclei in red; areas with cavitations are marked in yellow dotted line; sections from vehicle injected animals are marked with an asterisk; Fig. VII-7A).

Grafted NSI-566RSCs were found to widely express human-specific synaptophysin (h.Syn in blue; Fig. VII-7B). At one and a half month prior to sacrifice, cortico- and rubrospinal axons (in the posterior funiculi and the posterior side of the lateral funiculi of rat spinal cords, respectively) were labeled anterogradely with Green Fluorescent Protein (GFP in green; Fig. VII-7A). Human Neuron-Specific Enolase immunoreactive neurites were observed in between host long descending motor axons (h.NSE/human neurites in red, host axons in green; Fig. VII-7C). Also, confocal imaging showed human-specific synaptophysin immunoreactive synapses bordering on long descending host motortract axons and/or human-specific neurofilament antibody immunoreactive axons (h.Syn in blue; HO14 in red; Fig. VII-7D\&E). Last, human-specific GFAP immunoreactive astroglia cells (in red; with verified specific colocalization with h.NuMA immunoreactivity, in green; Fig. VII-7F) were widely present near the grafted lesion site in spinal cord of NSI-566RSC-grafted animals, but no Olig2immunoreactive cells were found to colocalize with h.NuMA-immunoreactive cells.

In conclusion, our immunohistochemical analyses, albeit based on a limited number of animals, indicates that i) in vivo differentiation of grafted NSI-566RSCs into neuronal and astroglial lineages occurred, ii) there was a permissive environment for growth of neurites and formation of synapses from grafted NSI-566RSCs in the host spinal cord, and iii) synapses are formed from NSI-566RSCs which were found bordering on host descending spinal axons.

\section{Discussion}

This study showed that over nearly ten months after high dose spinal NSI-566RSC-engraftment in spinal cord injured immunodeficient rats: i) no signs of NSI-566RSC-engraftment-related toxicity were noted, and specifically, ii) no definitive evidence of tumor growth related to NSI-566RSCengraftement was found within the spinal cords, brains, nor any other organs, and iii) no adverse 
health observations occurred which could be related to the NSI-566RSC-engraftement. All major health issues that occurred during the study were all related to the use of immunodeficient rats, the injury and/or surgery, as well as observations seen with aging rats. Moreover, iv) no decline in longterm neurologic function was observed after high dose spinal NSI-566RSC-engraftment, and v) histological assessment showed that spinally grafted NSI-566RSCs integrated in the host tissue as they were a) present in substantial numbers at the lesion site and at sites more distant from the lesion site, b) differentiated in neuronal and astroglial cells, c) extended into the host spinal cords, and d) formed synapses with host cells, including synapses on long descending axons from the Cortico- and Rubrospinal Tracts.

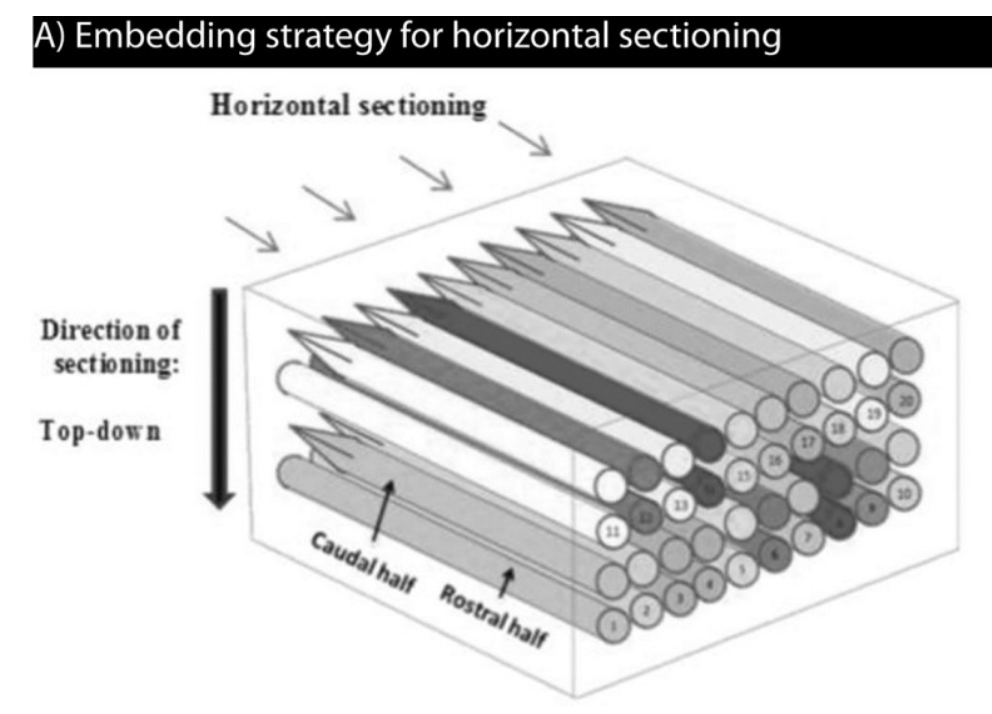

\section{B) Spinal cord division relative to graft/injury}

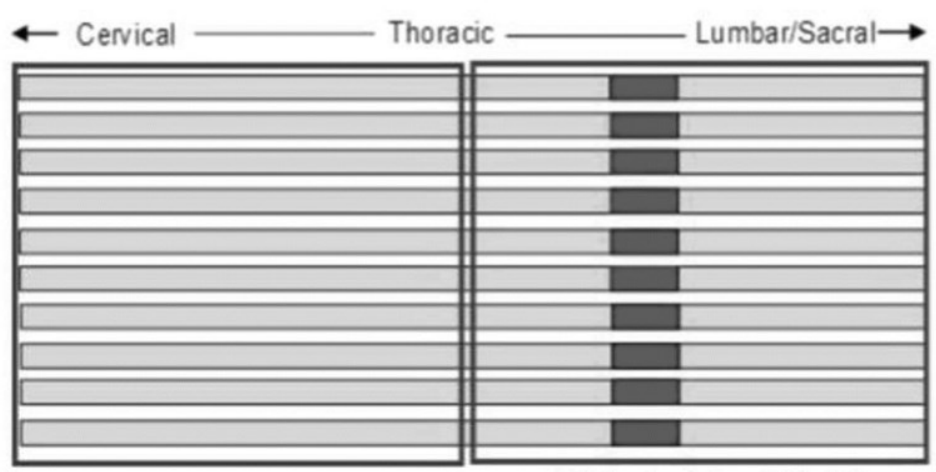

Figure VII-8: Embedding strategy for preparation of external histopathological assessment of spinal cords. A) Full spinal cords $(\sim 11 \mathrm{~cm})$ were divided in pieces of equal length. The pieces were then mounted above each other in gelatin together with 17 other, similarly divided, cords. B) The location of the injury in the spinal cord and its relative position to the separation cut used for the gelatin embedding, as shown in Figure $A$.

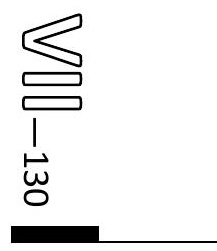


Some major considerations for the design of a safety study for cell-based therapies have not yet been discussed. First, as there is no predefined cell dose for safety assessments, an optimal cell dose needs to be determined. Although it is recommended to use high doses of any cell-based therapy for safety assessments, it is important to note that if tumors and/or an increased morbidity are then found, the whole study might have to be repeated with a lower dose group. Too high concentrations of growth inducing cells, could lead to confounding morbidity/mortality in the toxicity study, not in the least due to host tissue unable to accommodate more engrafted cells (i.e. not due to therapeutic properties, but physical and/or metabolic effects) (Mannello 2011). Timing of cell-grafting was chosen at one week post injury to prevent pronounced metabolic competition between host tissue and grafted cells, which otherwise would occur in the acute phase post injury. While normal, most stem cells tend to proliferate after engraftment, like NSI-566RSCs, effectively increasing their initial cell dose, which can also cause physical problems. Moreover, injections of human neural stem cells into contusion rat spinal cords led to increased proliferation of the cells, beyond the level observed in intact tissue, as observed in our preliminary studies [unpublished data]. Inclusion of an intermediate-dose and a normal-dose group can be a safety net for toxicity studies. However, in our case, spinal lesioning and (delayed) intraspinal grafting of cells are laborious surgeries, which can quickly result in limitations to the amount of animals in a single study. The cost can also be prohibitive, not in the least since safety studies need to be carried out in a GLP-compliant contract research organization's facility over a considerable time period. Therefore, it is advisable to conduct preliminary studies (in target tissue and subcutaneously) to estimate the maximal feasible dose, during which a reasonable confidence is gained for the appropriate cell dose for the safety assessment.

Secondly, the animal model requires further considerations. Logically, the use of athymic animals, necessary for safety assessments in cell-based therapies, limits the availability of concurrent disease modeling. In our case, although the IND application of NSI-566RSCs was for a trial in ALS-patients, the spinal cord injury model is useful for the safety assessment for this application by offering spinal tissue with varying severities of spinal tissue disease/injury. Tumorigenicity was assessed in injured tissue near the epicenter up to fairly uninjured, more remote tissue, and thus provides a broad and sensitive assay to test for tumorigenicity. Having many unscheduled deaths due to disease modeling is also impractical and unethical, which is another reason for not using ALS models. It is also why in our study a moderate spinal cord injury model was chosen. In such a way the injury would barely lead to increased mortality and animals would soon recover spontaneous bladder functions and adequate locomotion (within several weeks) (Basso 1996). As a rule of thumb, a study design should not anticipate $>25 \%$ mortality, even in case of natural mortality. In this perspective a 9-10 months 
survival in 2-3 months old athymic animals remained feasible, as longevity of athymic rat has been reported to be 9-18 months (Festing 1982, Schuurman 1995). Rats were chosen because athymic animals are available, dosage can be higher than in mice, nude mice are more susceptible to infections than nude rats (van Loveren 1988), and most efficacy data of NSI-566RSCs was established in rats. Only male rats were used in this study, but there is no basis to suspect tumorigenicity of hNSCs is sex-dependent. In our experience, male rats tend to have mildly higher tendencies towards morbidity after $\mathrm{SCl}$, thus possibly yielding higher/better toxicity sensitivity.

Regarding the therapeutic efficacy of NSI-566RSCs in this study, it should be emphasized that due to the design of the study, entailing mainly the large loading dose of NSI-566RSCs and amount of injections needed for the toxicity assessment, any therapeutic efficacy of the NSI-566RSCengraftment was not expected. Nevertheless, NSI-566RSCs showed good histological integration and both neuronal and glial differentiation. Furthermore, albeit the gross morphology of the spinal cords around the lesion sites was qualitatively similar in spinal cords of rats that received injury plus vehicle-only as compared to those that received contusion injury plus NSI-566RSCs, the lesions tended to be more severe in control rats (i.e. more traumatic tissue destruction) than in NSI-566RSCengrafted rats (i.e. mostly subacute tissue degeneration), suggesting a possible healing response via structural improvement in the cell injected rats (Wirth 2001). Traumatic tissue destruction, however, is somewhat similar to subacute degeneration only apart from a greater degree of actual volume loss, i.e. with a substantial narrowing of the circumference of the spinal cord at that site. The traumatic tissue destruction as observed mostly in vehicle-injected rats is therefore difficult to consider separately from subacute tissue degeneration after (voluminous) cell therapy has been applied. Another possible positive effect of NSI-566RSCs in this study seems to be the prevention of a glial scar and syringomyelia, as any increase in cell density near the injury site of vehicle control animals was mainly attributed to astroglial cells/scarring, while in NSI-566RSC-engrafted animals cell density increases were generally due to the presence of grafted cells filling up the cavity. This indicates a possible limiting effect on the glial scar and syrinx formation in NSI-566RSC-engrafted animals.

In addition, with respect to the results of NSI-566RSC on the neurological function assays, it should be noted that while positive treatment effects appear to be approaching significance in some cases, the effect sizes would be small. Gait and activity analyses provide many variables for analysis, but only those of relevance in spinal cord injury were analysed. CatWalk gait-analyses prove especially useful to test spinal cord function through objectively scoring of coordinated locomotion (i.e. smooth transitions between front and hind paws/limbs, with in-line placements of the front and 
hindpaws) (Hamers 2006). These measures reflect the function of descending tracts, and hence, neuroprotection and or regeneration. This measure is best represented by the variables regularity index and hind paw base of support (Hamers 2001, Hamers 2006). Group comparisons for gaitanalyses require similar speed and hindpaw work in animals between groups. These factors were controlled through the measurement of run duration, and the stride length, and stance and swing duration of the hindpaws (Hamers 2006). Activity analyses were used to assess differences in variables relevant to spinal function, i.e. movement leading to actual displacements, in terms of distance moved, frequency of such movement, and velocity/durations. None of the motor analyses showed significant differences between groups. Last, no effects on hindpaw sensory function (i.e. pain perception) were noted. Due to the lack of control animals/measurements, any trends in differences in sensory function could reflect either an effect in hyperalgesia, allodynia and/or hypesthesia.

The data from this study and the abovementioned efficacy and safety studies were used for IND application and successfully led to IND approval of NSI-566RSC. This study can function as a reference guide in the development of future safety studies of cell-based therapies. Nonetheless, it remains important to keep supervising drug authorities (e.g. Office of Gene and Cell Therapy/CBER/FDA) involved at an early phase of a product development program, to enable early identification of potential individual issues and solutions. 


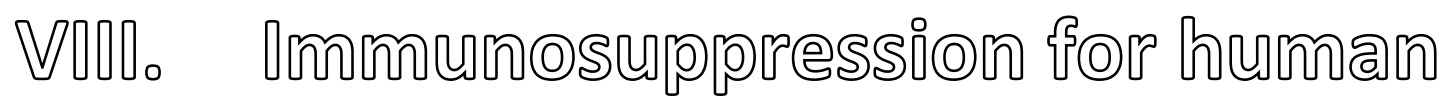 ๔ell-based therapi̊es i̊n i̊mmunocompe-

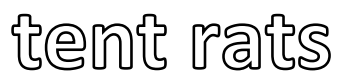




\title{
Effective long-term immune-suppression in rats by
}

\section{subcutaneously implanted sustained-release tacrolimus} pellet: effect on spinally grafted human neural precursors

\section{survival.}

\author{
Juraj Sevc, Danielle Goldberg, Sebastiaan van Gorp, \\ Marjolein Leerink, Stefan Juhas, Jana Juhasova, Silvia Marsala, Marian Hruska-Plochan, Michael \\ Hefferan, Jan Motlik, Frantisek Rypacek, Ludka Machova, Osamu Kakinohana, Camila Santucci, \\ Karl Johe, Nadezda Lukacova, Kazuhiko Yamada, Jack Bui, Martin Marsala
}

Experimental Neurology

\begin{abstract}
Achievement of effective, safe and long-term immunosuppression represents one of the challenges in experimental allogeneic and xenogeneic cell and organ transplantation. The goal of the present study was to develop a reliable, long-term immunosuppression protocol in Sprague-Dawley (SD) rats by: A) comparing the pharmacokinetics of four different subcutaneously delivered/implanted tacrolimus (TAC) formulations, including: 1) caster oil/saline solution, 2) unilamellar or multilamellar liposomes, 3) biodegradable microspheres, and 4) biodegradable 3-month lasting pellets; and B) defining the survival and immune response in animals receiving spinal injections of human neural precursors at 6 weeks to 3 months after cell grafting. In animals implanted with TAC pellets (3.4 $\mathrm{mg} / \mathrm{kg} / \mathrm{day}$ ), a stable 3-month lasting plasma concentration of TAC averaging $19.1 \pm 4.9 \mathrm{ng} / \mathrm{ml}$ was measured. Analysis of grafted cell survival in SOD+ or spinal trauma-injured SD rats immunosuppressed with 3-month lasting TAC pellets (3.4-5.1 mg/kg/day) showed the consistent presence of implanted human neurons with minimal or no local T-cell infiltration. These data demonstrate that the use of TAC pellets can represent an effective, long-lasting immunosuppressive drug delivery system that is safe, simple to implement and is associated with a long-term human neural precursor survival after grafting into the spinal cord of SOD+ or spinal trauma-injured SD rats.
\end{abstract}




\section{Introduction}

One of the essential requirements for successful translation of experimentally-defined cell-based replacement therapies which utilize the allogeneic or xenogeneic cell grafts into clinical practice is the development of safe and effective immunosuppression protocols that will permit long-term survival and maturation of grafted cells. Current clinical and experimental immunosuppression protocols typically use single or combined immunosuppressive drug regimens with drugs delivered orally, intraperitoneally, intravenously or subcutaneously in a single daily dose or divided into multiple daily doses [see reviews (Barraclough 2011, Halloran 1996, MacGregor 1995, Wente 2006)]. While in human clinical settings a targeted plasma concentration of a variety of immunosuppressant drugs can effectively be achieved by a drug dose titration, to accomplish comparable consistency in targeted plasma levels in animal studies remains a major challenge.

Besides cyclosporines, mycophenolate mofetil (MMF), rapamycin or prednisolone, TAC (FK-506, Prograf) represents an immunosuppressant of choice and is frequently used as a solo therapy or in combination with other immunosuppressive drugs (i.e., MMF) (Hefferan 2011, Reis 1998, Xu 2010) [see reviews (Lama 2003, Su 2011)]. TAC couples with immunophilins, proteins termed FK-506 binding proteins (FKBPs) (Siekierka 1989, Thomson 1995). The formation of a pentameric complex comprised of TAC, FKBPs, calcineurins $A$ and $B$ and calmodulin results in the inhibition of the phosphatase activity of calcineurin (Halloran 1996, McKeon 1991). The action of transcription factors requiring dephosphorylation for transport to the cell nucleus is inhibited and leads to suppression of T-cell proliferation and function (Thomson 1995).

In human clinical allogeneic organ transplantation, the recommended concentration of TAC in blood is in the range of $10-20 \mathrm{ng} / \mathrm{ml}$ (Pirsch 1997, Przepiorka 1999, Staatz 2004) and is effective in maintaining long-term survival of transplanted solid organs (such as kidney, bone marrow or liver) with tolerable side effects typically presented as nephrotoxicity, neurotoxicity, gastrointestinal toxicity or drug-induced diabetes (Vicari-Christensen 2009). In experimental allograft or xenograft animal studies that use rodents (mice, rats) or minipigs as recipients, TAC is typically administered using a chronically implanted intravenous catheter, intraperitoneally or subcutaneously, with doses ranging from 0.05-3 mg/kg/24 hrs (Gold 1995, Hefferan 2011, Saxena 2007, Tze 1992, Usvald 2010). However, despite the use of such aggressive immunosuppressive protocols, experimental xenograft studies are frequently hampered by inconsistent graft survival particularly seen in long-term survival studies. It is believed that the oscillation in plasma drug concentrations and/or insufficient target plasma levels may in part account for inconsistent graft survival. In addition, the requirements of BID (fom latin "bis in die": two times a day) injections in order to achieve satisfactory TAC levels and to 
minimize toxicity make this approach 1) labor intensive, 2) frequently associated with side effects resulting from repetitive animal injections (such as local inflammatory changes and infection), and 3) associated with systemic side effects such as nephrotoxicity and hepatotoxicity [see reviews (Finn 1999, Gijsen 2010, Teh 2011)].

To extend the half-life of administered drugs in general, several longer-releasing formulations were developed. First, the use of TAC-loaded liposomes has been shown to provide moderate prolongation of the TAC half-life in the whole blood of naïve rats in comparison with conventional i.v. injections of TAC diluted in saline (Ko 1994, McAlister 1998). Second, the use of biodegradable microspheres was shown to provide a relatively stable level of TAC in whole blood for up to 10-21 days after single s.c. administration (Miyamoto 2004, Wang 2004). Third, the use of implantable biodegradable pellets has been successfully used to deliver a variety of synthetic drugs or hormones in human patients and in animal experimental models and showed up to 3-6 months of stable drug release after a single pellet implantation (Jockenhovel 1996, Packard 1992, Srinivasan 2002, Studd 1987). To our knowledge, no immunosuppressive pellet formulation has been reported to be successfully used in rodent or other animal models of xenogeneic neural precursor transplantation.

Accordingly, the goal of the present study was two-fold. First we characterized the pharmacokinetics of four different subcutaneously delivered/implanted TAC formulations, including: 1) caster oil/saline solution, 2) unilamellar or multilamellar liposomes, 3) biodegradable microspheres, and 4) biodegradable 3-month lasting pellets. The optimal TAC formulation, as defined by simplicity of TAC delivery and stable/predictable blood TAC concentration was then selected and used in the second component of our study. The primary goal of the second part of the study was to validate the level of functionally effective immunosuppression in a separate group of SOD $1^{\mathrm{G} 93 \mathrm{~A}}$ transgenic or spinal trauma-injured SD rats implanted with 3-month lasting TAC pellets and grafted spinally with human fetal spinal stem cells (hSSC) or human ES-derived neural precursors (ES-NPC). The survival of grafted cells was determined at 1-3 months after grafting using human-specific antibodies and confocal microscopy.

In addition, the potency of TAC pellet-induced immunosuppression was validated by quantitative analysis of the circulating T-cell population (CD45, CD4, CD8) and by qualitative and quantitative analysis of the infiltrating T-lymphocytes (CD45, CD4, CD8) in cell-grafted spinal cord regions.

Our results indicate that s.c. implanted 3-month lasting biodegradable TAC pellets represent an effective, safe and simple method to achieve long lasting and effective immunosuppression as 
evidenced by 1) consistent xenograft survival and cell maturation, 2) near complete suppression of grafted site T-cell infiltration, and 3) suppression of circulating blood T-cell concentration.

\section{Material and Methods}

All procedures were approved by the Institutional Animal Care and Use Committees by the University of California, San Diego and by the Czech Academy of Sciences. Adult Sprague-Dawley

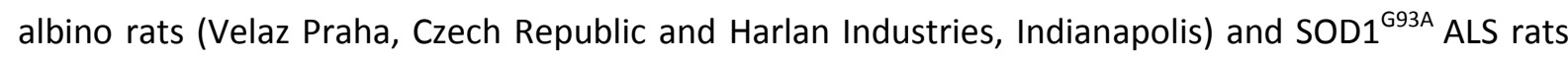
(SOD+) (UCSD colony, Dr. D. W. Cleveland, San Diego, California; 49-57 days old) were used in experiments. Animals were housed in standard cages with free access to food and water.

Animal experimental groups were divided into 2 principal studies: 1) TAC pharmacokinetic study, and 2) spinal grafting of human neural precursors in TAC pellet-immmunosuppressed animals.

\section{TAC pharmacokinetic study}

Four different TAC (Prograf ${ }^{\circledR}$, Astellas Pharma, Deerfield, Illinois, USA) vehicle-delivery systems were used and delivered into the subcutaneous space (see Table VIII-1 for summary).

\begin{tabular}{|c|c|c|c|c|c|c|}
\hline Group & Route & Vehicle & Dose & Frequency & $\mathrm{n}$ & $\begin{array}{l}\text { Time points } \\
\text { (detection of blood- } \\
\text { levels) }\end{array}$ \\
\hline No.1 & $\begin{array}{l}\text { s.c. } \\
\text { injection }\end{array}$ & $\begin{array}{l}\text { powder dissolved in caster } \\
\text { oil/saline solution }\end{array}$ & $\begin{array}{l}3 \\
\mathrm{mg} / \mathrm{kg}\end{array}$ & every $24 \mathrm{~h}$ & 4 & $2,9,24,72,120$ hours \\
\hline No.2 & $\begin{array}{l}\text { s.c. } \\
\text { injection }\end{array}$ & $\begin{array}{l}\text { powder dissolved in caster } \\
\text { oil/saline solution }\end{array}$ & $\begin{array}{l}1.5 \\
\mathrm{mg} / \mathrm{kg}\end{array}$ & every $12 \mathrm{~h}$ & 4 & $2,12,14,24,120$ hours \\
\hline No.3 & $\begin{array}{l}\text { s.c. } \\
\text { injection }\end{array}$ & unilamellar liposomes & $\begin{array}{l}3 \\
\mathrm{mg} / \mathrm{kg}\end{array}$ & $\begin{array}{l}\text { once, } \\
\text { at beginning }\end{array}$ & 4 & $2,12,24,48,72$ hours \\
\hline No.4 & $\begin{array}{l}\text { s.c. } \\
\text { injection }\end{array}$ & multilamellar liposomes & $\begin{array}{l}3 \\
\mathrm{mg} / \mathrm{kg}\end{array}$ & $\begin{array}{l}\text { once, } \\
\text { at beginning }\end{array}$ & 4 & $2,12,24,48,72$ hours \\
\hline No.5 & $\begin{array}{l}\text { s.c. } \\
\text { injection }\end{array}$ & microspheres & $\begin{array}{l}10 \\
\mathrm{mg} / \mathrm{kg}\end{array}$ & $\begin{array}{l}\text { once, } \\
\text { at beginning }\end{array}$ & 3 & $\begin{array}{l}2,9,24 \text { hours, } \\
2,4,7,10,13,16,19,22 \\
\text { days }\end{array}$ \\
\hline No.6 & $\begin{array}{l}\text { s.c. } \\
\text { injection }\end{array}$ & microspheres & $\begin{array}{l}20 \\
\mathrm{mg} / \mathrm{kg}\end{array}$ & $\begin{array}{l}\text { once, } \\
\text { at beginning }\end{array}$ & 3 & $\begin{array}{l}2,9,24 \text { hours, } \\
2,4,7,10,13,16,19,22 \\
\text { days }\end{array}$ \\
\hline No.7 & $\begin{array}{l}\text { s.c. } \\
\text { implant }\end{array}$ & 3 months lasting pellet & $\begin{array}{l}1.8 \pm 0.15 \\
\mathrm{mg} / \mathrm{kg} / \mathrm{day}\end{array}$ & $\begin{array}{l}\text { once, } \\
\text { at beginning }\end{array}$ & 4 & $\begin{array}{l}5,13,20,29,50,56,71,90 \\
\text { days }\end{array}$ \\
\hline No.8 & $\begin{array}{l}\text { S.c. } \\
\text { implant }\end{array}$ & 3 months lasting pellet & $\begin{array}{l}3.43 \pm 0.21 \\
\mathrm{mg} / \mathrm{kg} / \mathrm{day}\end{array}$ & $\begin{array}{l}\text { once, } \\
\text { at beginning }\end{array}$ & 3 & $\begin{array}{l}5,7,10,17,26,38,59,74 \\
90105,120,140 \text { days }\end{array}$ \\
\hline No.9 & $\begin{array}{l}\text { S.C. } \\
\text { implant }\end{array}$ & 3 months lasting pellet & $\begin{array}{l}5.1 \pm 1 \\
\mathrm{mg} / \mathrm{kg} / \text { day }\end{array}$ & $\begin{array}{l}\text { once, } \\
\text { at beginning }\end{array}$ & 6 & $10,27,42,53$ days \\
\hline
\end{tabular}

Table VIII-1 Summary of experimental groups used in tacrolimus PK study. 


\section{1) TAC caster oil/saline solution (Groups No. 1 and No. 2):}

Because the hydrophobic nature of TAC powder and its poor solubility in water solutions (e.g., saline) (Kino 1987), TAC powder was dissolved in a mixture of $100 \%$ ethanol ( $8 \%$ of total volume), caster oil ( $2 \%$ of total volume) and sterile saline for injections ( $90 \%$ of total volume; Fig. VIII-1 G). Two dosing designs were studied. In the first dosing design, animals ( $n=4 ;$ Grp. No. 1 ) received 3 $\mathrm{mg} / \mathrm{kg}$ of TAC in 24-hr intervals for a total of 5 days. Blood samples for TAC measurement were collected at 2, 9, 24, 72 and $120 \mathrm{hrs}$. At 24 and $72 \mathrm{hrs}$ the blood samples were collected just before subsequent TAC injection. In the second dosing design, animals ( $n=4$; Grp. No. 2) received $1.5 \mathrm{mg} / \mathrm{kg}$ of TAC in 12-hr intervals for a total of 5 days. Blood samples for TAC measurements were collected at 2, 12, 14, 24 and $120 \mathrm{hrs}$. At 12 and $24 \mathrm{hrs}$ the blood samples were collected just before subsequent TAC injections.

\section{2) TAC liposomes (Groups No. 3 and No. 4):}

Two structurally different liposome designs (unilamellar or multilamellar; Fig. VIII-1 I, J) were used (Encapsula NanoSciences LLC, TN). In the first group, TAC-loaded unilamellar liposomes $(n=4 ; 3$ $\mathrm{mg} / \mathrm{kg}$; Grp. No. 3) were used. In the second group, TAC-loaded multilamellar liposomes ( $\mathrm{n}=4 ; 3$ $\mathrm{mg} / \mathrm{kg}$; Grp. No. 4) were used. In both groups, TAC liposomes were injected as a single bolus. Blood samples for TAC measurements were collected at 2, 12, 24, 48 and $72 \mathrm{hrs}$.

\section{3) TAC microspheres (Groups No. 5 and No. 6):}

TAC-containing microspheres were prepared from tacrolimus powder and poly ( $D, L$-lactidecoglycolide) copolymer (Resomer LG 503H, Aldrich) adopting the procedure previously described (Wang 2004). The tacrolimus content in the resulting dry TAC microspheres was $45 \mathrm{mg}$ TAC/g of microspheres as determined by HPLC. Rats were injected with a single bolus of TAC-containing microspheres at a dose of $10 \mathrm{mg} / \mathrm{kg}$ ( $n=3$; Grp. No. 5) or $20 \mathrm{mg} / \mathrm{kg}$ ( $n=3$; Grp. No. 6). Blood samples for TAC measurements were collected at 2, 9, $24 \mathrm{hrs}$ and at 2, 4, 7, 10, 13, 16, 19, and 22 days. 


\section{4) TAC releasable pellets (Groups No. 7, No. 8 and No. 9):}

TAC-containing 3-month releasable pellets $(45,65$ or 90 mg/pellet; Innovative Research of America, FL, USA or Tacropellet, MD, USA; Fig. VIII-1H) were implanted subcutaneously in the interscapular region of the neck in isoflurane (2\%)-anesthetized rats. Animals with three different body weight ranges (120-350 g) were employed resulting into 3 different dosing groups releasing $1.8 \mathrm{mg} / \mathrm{kg} / 24$ hrs ( $n=4$; Gr. No. 7), 3.4 mg/kg/24 hrs (n=3; Grp. No. 8) and 5.1 mg/kg/24 hrs ( $n=6$; Grp. No. 9) of TAC. Blood samples for TAC measurements were collected periodically between 5-140 days after TAC pellet implant (see Table VIII-1 for details).

\section{Blood collection and TAC analysis:}

In all experimental groups, blood samples were collected from the saphenous vein in fully awake restrained animals. The lateral aspect of the hind leg was shaved, cleaned with $70 \% \mathrm{EtOH}$ and disinfected with Chlorhexidine. The leg was immobilized in the extended position by applying gentle downward pressure immediately above the knee joint. Petroleum jelly was applied on the site in order to visualize the saphenous vein and to optimize the blood flow. A 20 gauge needle was used to puncture the vein. We first punctured the vein proximal to the ankle and then moved up if additional punctures were necessary (no more than three needle punctures were attempted in any of blood collection sessions). A maximum of $200 \mu$ l of blood was collected per time point. Blood was collected into EDTA tubes and kept at $-20^{\circ} \mathrm{C}$ until analysis using 2 equivalent validated assays: chemiluminescent microparticle immunoassay (CMIA) and liquid chromatography/mass spectrometry (LC/MS/MS).

Chemiluminescent microparticle immunoassay (CMIA). CMIA - ARCHITECT TAC (Abbot Architect, Chicago, Illinois, USA) assay was used according to the manufacturer's protocol. The detection limits of quantification for this method were $2 \mathrm{ng} / \mathrm{ml}(\min )$ and $30 \mathrm{ng} / \mathrm{ml}$ (max), respectively. If the concentration of TAC in the sample exceeded the $30 \mathrm{ng} / \mathrm{ml}$ limit, blood was diluted with saline (in a 1:1 ratio) and the measurement repeated. The CMIA method was used for quantification in groups $1,2,3,4$ and 8 .

High performance liquid chromatography - mass spectrometry (LC/MS/MS).

A guard column C18, $4 \times 2.0$ mm (Phenomenex, Torrance, California, USA) equipped with ABI 4000 QTrap linear ion trap mass spectrometer ( $A B$ Sciex, Concord, Canada) was used. Whole blood samples were prepared as follows: $40 \mu \mathrm{l}$ of sample (EDTA whole blood) was mixed with $140 \mu \mathrm{l}$ protein precipitation solution (conc. ascomycin and zinc sulfate dissolved in methanol). After centrifugation $(17,000 \times \mathrm{g}$ for $5 \mathrm{~min}$.), $40 \mu \mathrm{l}$ of the supernatant was transferred to autosampler vials 
for injection into the LC/MS/MS system. Guard column C18 was washed for $1 \mathrm{~min}$. (isocratic flow rate, $600 \mu \mathrm{l} / \mathrm{min}$.) with a mixture of methanol containing $2.5 \mathrm{mM} / \mathrm{l}$ ammonium acetate and distilled water containing $2.5 \mathrm{mM} / \mathrm{l}$ ammonium acetate (ratio 75:25). Thereafter, the mobile phase was changed to $2.5 \mathrm{mM}$ ammonium acetate dissolved in methanol (100\% of the volume, flow rate 600 $\mu \mathrm{l} / \mathrm{min}$., time $1.50 \mathrm{~min}$ ) and TAC/ascomycin were eluted to the detector. The column was reconditioned with methanol containing $2.5 \mathrm{mM} / \mathrm{l}$ ammonium acetate $(75 \%$ of the volume, flow rate $600 \mu \mathrm{l} / \mathrm{min}$., time $1.50 \mathrm{~min})$. MS/MS analysis was performed in multiple reactions monitoring mode using transactions $\mathrm{m} / \mathrm{z}$ 821.6>768.5 for TAC and $\mathrm{m} / \mathrm{z}$ 809.5>756.5 for ascomycin. System control and data acquisition were performed using Analyst 1.2 software (Applied Biosystems/MDS Sciex) for automated data processing. The detection limits of quantification for this method were $2 \mathrm{ng} / \mathrm{ml}$ $(\min )$ and $50 \mathrm{ng} / \mathrm{ml}(\max )$, respectively. LC/MS/MS method was also used for determination of concentration of the TAC in whole blood in groups 5, 6, 7 and 9.

\section{Assessment of Tacrolimus pellets - induced side effects:}

Animals were evaluated daily for the presence of motor dysfunction (upper extremity motor function assessment was used in paraplegic spinal trauma animals), agitation, jumpiness, and tactile allodynia. In addition, animals were monitored for the presence of other potentially TAC-treatmentrelated signs of toxicity including: scruffy coat, porphyrin (an indicator of stress, known as "red tears", often mistaken as blood), weight loss, hypothermia, pale eyes (a reliable indicator of anemia), soft stool, diarrhea, lethargy, vocalization, and swollenness of the pellet implanted subcutaneous site (potential indicator of a local cyst formation).

Spinal cord grafting of human fetal tissue-derived stem cells or embryonic cell line-derived neural precursors

To assess the effectiveness of immunosuppression in TAC pellet-immunosuppressed rats, human fetal spinal cord derived stem cells (hSSC) or human embryonic cell line (HUES-7)-derived neural precursors (HUES7-NPC) were employed for intraspinal grafting in transgenic SOD+, $(n=10)$ rats or in SD rats with previous L3 compression injury $(n=4)$ (see Table VIII-2 for experimental cell grafting groups).

Derivation of both cell lines was described in detail in our previous studies (Johe 1996, Kakinohana 2012, Usvald 2010, Yuan 2011). Briefly, human fetal spinal stem cells (Neuralstem, Inc., Rockville, Maryland, USA) were derived from the cervical-upper thoracic region of spinal cord tissue obtained from a single 8-week human fetus after an elective abortion. One day prior to each surgery day, one cryopreserved vial of the previously prepared neural precursors was washed, concentrated in hibernation buffer, and shipped from the cell preparation site (Neuralstem, Inc., Rockville, MD, USA) 
to the surgery site (UCSD, San Diego, CA, USA) at $2-8^{\circ} \mathrm{C}$ by overnight delivery. Upon receipt the following day, the cells were used directly for implantation without further manipulation. Before and after implantation, the viability of cells was measured with trypan blue (0.4\%; Sigma). On average, $88-93 \%$ viability was seen.

\begin{tabular}{ccccc}
\hline Rat strain & Group & Animal ID & Grafted cell line & $\begin{array}{c}\text { Survival time after cell } \\
\text { grafting (days) }\end{array}$ \\
\hline SOD1 (ALS) & No.7 & 4746 & HUES7 & 77 \\
SOD1 (ALS) & No.7 & 4753 & HUES7 & 33 \\
SOD1 (ALS) & No.7 & 4750 & NSI & NSI \\
SOD1 (ALS) & No.7 & 4752 & HUES7 & 77 \\
SOD1 (ALS) & No.9 & 5038 & HUES7 & 40 \\
SOD1 (ALS) & No.9 & 5081 & NSI & 32 \\
SOD1 (ALS) & No.9 & 5041 & HUES7 & 38 \\
SOD1 (ALS) & No.9 & 5076 & NSI & 32 \\
SOD1 (ALS) & No.9 & 5095 & NSI & 70 \\
SOD1 (ALS) & No.9 & 5104 & HUES7 & 37 \\
SD (SCCI) & - & 149 & HUES7 & 105 \\
SD (SCCI) & - & 153 & HUES7 & 105 \\
SD (SCCI) & - & 155 & HUES7 & 105 \\
SD (SCCI) & - & 160 & 105 \\
\hline
\end{tabular}

Table VIII-2: Experimental cell grafting groups. $\mathrm{SCCl}$ = spinal cord contusion injury

The human embryonic stem cells (hESCs; HUES-7 line; Melton Laboratory, Harvard University, Massachusetts, USA), were cultured on a mitomycin C-treated mouse embryonic fibroblast (MEF) feeder layer in HUES hESC medium. Columnar rosettes were manually isolated from induced embryoid bodies and passaged every 3 days to remove contaminating cells. In this stage, NPC were harvested and FAC-sorted (Fluorescence-activated cell sorting-FACS) and CD184+, CD44-, CD271-, CD24+ cell populations further expanded on PLO/L- coated plates using modified N2 media. For cell growth, $10 \mathrm{ng} / \mathrm{ml} \mathrm{bFGF}$ as the sole mitogen was added. NPC were expanded for 10-20 passages and frozen aliquots prepared from passage 15-20. On surgery day, one cryopreserved vial of the previously prepared passage was thawed, washed and concentrated in hibernation buffer. Before and after implantation, the viability of cells was measured with trypan blue (0.4\%; Sigma). On average, $85-95 \%$ viability was seen. 


\section{Spinal cord cell-grafting procedure}

SOD+ rats: animals weighing 267 \pm 20.9 g (Group No. 7, age 57 days; $n=4$; Table VIII-1 and Table VIII2) and 203.6 \pm 44 g (Group No. 9, age 49-52 days; $n=6$; Table VIII-2) received spinal grafts of hSSC or HUES7- NPC at 13 days after TAC pellet implantation. To implant cells, the previously described technique was used (Kakinohana 2004). Rats were anesthetized with isoflurane ( $2 \%$ maintenance in room air), placed into a spinal unit apparatus (Stoelting, Wood Dale, Illinois, USA) and a partial Th12L1 laminectomy was performed using a dental drill (exposing the dorsal surface of L2-L5 segments). Using a 33-gauge needle connected to a microinjector (Kopf Instruments, Tujunga, California, USA), the spinal cord was injected with $1 \mu \mathrm{l}$ (approx. 15,000 cells per injection) of the hSSC or HUES7- NPC cells in hibernation buffer. The duration of each injection was $60 \mathrm{~s}$ followed by a $30 \mathrm{~s}$ pause before needle withdrawal. The center of the injection was targeted into the base of the ventral horn. 10 injections (approx. $800 \mu \mathrm{m}$ rostrocaudally apart) were made on the left side of the lumbar spinal cord. After injections, the incision was cleaned with penicillin-streptomycin solution and sutured in two layers. Animals were injected with analgesics and antibiotics and were allowed to recover. For the first 14 days after transplantation, additional immunosuppression was performed as previously described (Hefferan 2011) with daily injection of mycophenolate mofetil (MMF, Cellcept, Roche Pharmaceutical, Nutley, New Jersey, USA, dose $30 \mathrm{mg} / \mathrm{kg}$ ). Animals were allowed to survive until they reached the endstage of the disease (Group 7; 33-77 days after cell grafting, for details see Table VIII-2) or until they lost $10 \%$ of their bodyweight caused by the progression of the disease (Group 9; 32-70 days after cell grafting, for details see Table VIII-2).

Spinal cord (L3) contused rats: four female SD rats ( $n=4$; Group No.10; BW: $205 \pm 11.9$ g) were anesthetized with isoflurane ( $2 \%$ maintenance in room air), placed into a spinal unit apparatus (Stoelting, Wood Dale, Illinois, USA) and a partial Th13 laminectomy was performed using a dental drill (exposing the dorsal surface of L3 spinal segment). Spinal cord compression injury was induced by placing a $3.2 \mathrm{~mm}$ acrylic rod (weight=32 $\mathrm{g}$ ) for $15 \mathrm{~min}$ on the dorsal surface of the exposed L3 segment. After compression, the incision was cleaned with penicillin-streptomycin solution and sutured in two layers. Animals were injected with Depomedrol (methylprednisolone, $10 \mathrm{mg} / \mathrm{kg}$ ) and antibiotics (Cefazoline, $10 \mathrm{mg} / \mathrm{kg}$ ) and were allowed to recover. Three days after injury animals were re-anesthetized, previously injured L3 spinal segment exposed and received spinal grafts (total of 5 injections; $1 \mu \mathrm{l}$ per injection, approx. $400 \mu \mathrm{m}$ rostrocaudally apart) of HUES7-NPC targeted into the epicenter and just above and below the injury. The following immunosuppression protocol was used: for the initial 14 days after cell grafting, animals received combined immunosuppression composed of daily single s.c. injection of MMF (30 mg/kg) and BID injections of TAC $(1.5 \mathrm{mg} / \mathrm{kg} / 12$ hrs). At 14 days, animals were implanted with TAC pellets delivering the TAC dose of $4.9 \pm 0.27$ 
$\mathrm{mg} / \mathrm{kg} /$ day. Because our initial PK study showed that on average 10-15 days is required to achieve a targeted plasma TAC concentration (i.e., above $15 \mathrm{ng} / \mathrm{ml}$ ), animals continued to receive BID injections of TAC ( $1.5 \mathrm{mg} / \mathrm{kg} / 12 \mathrm{hrs}$ ) for an additional 12 days. MMF treatment ( $30 \mathrm{mg} / \mathrm{kg} /$ day; s.c.) was only used for the initial 14 days after cell grafting. In this experimental group, animals survived 105 days after cell grafting (for details see Table VIII-2).

Naïve non-immunosuppressed SD rats: four female SD rats ( $n=4$; Group No.11; BW: $367 \pm 14 \mathrm{~g}$ ) received lumbar spinal grafts of hSSC (total of 5 injections; $1 \mu \mathrm{l}$ per injection, approx. $400 \mu \mathrm{m}$ rostrocaudally apart; approx. 15,000 cells per injection) as described. No immunosuppression was performed and animals survived for 14 days.

\section{Perfusion, fixation and immunofluorescence staining of spinal cord sections}

Rats were deeply anesthetized with pentobarbital and phenytoin and transcardially perfused with $200 \mathrm{ml}$ of heparinized saline followed by $250 \mathrm{ml}$ of $4 \%$ paraformaldehyde in PBS. The spinal cords were dissected and post-fixed in $4 \%$ formaldehyde in PBS overnight at $4{ }^{\circ} \mathrm{C}$ and then cryoprotected in $30 \%$ sucrose PBS until transverse or longitudinal sections (40- $\mu \mathrm{m}$-thick) were cut on a cryostat and stored in PBS. Sections were immunostained overnight at $4^{\circ} \mathrm{C}$ with the following primary humanspecific (h) or non-specific antibodies made in PBS with $0.2 \%$ Triton $\mathrm{X}-100$ : mouse anti-nuclear matrix protein/h-nuc (hNUMA; 1:100; Millipore, Temecula, California, USA), rabbit anti-human neuron-specific enolase (hNSE, 1:500, Chemicon), mouse anti-human synaptophysin (hSYN, Chemicon; 1:2000), rabbit anti-human glial fibrillary acidic protein (hGFAP, 1:500, Origene, Rockville, Maryland, USA), goat anti-doublecortin (DCX; 1:1000; Millipore), goat anti-choline acetyltransferase (CHAT, 1:50, Chemicon), mouse anti neuronal nuclei antigen (NeuN, 1:1000, Chemicon), mouse antiCD4 and anti-CD8 antibodies (1:500; AbD Serotec, Raleigh, North Carolina, USA), mouse anti-CD3 and anti-CD45 antibodies (1:1000, e-Bioscience, San Diego, California, USA), rabbit anti-lba1(IB1) (1:1000, Wako, Richmond, Virginia, USA), mouse anti-RT1B (MHC class II) (1:500, BD Biosciences, San Diego, California, USA), mouse anti CD11b (1:200, BD Biosciences, San Diego, California, USA) and donkey anti-rat IgG (1:500, Invitrogen). After incubation with primary antibodies, sections were washed three times in PBS and incubated with fluorescent-conjugated secondary donkey antimouse, donkey anti-rabbit or donkey anti-goat antibodies (Alexa Fluor 488, 546 or 647, 1:250, Invitrogen) and DAPI for general nuclear staining. Sections were then mounted on slides, dried at room temperature and covered with a Prolong anti-fade kit (Invitrogen). 


\section{Fluorescent microscopy and leukocyte quantification}

Fluorescence-stained sections were analyzed using a Leica DMLB Microscope with a Zeiss Axiocam MRm monochrome camera and Olympus FV1000 confocal deconvolution microscope. Images were captured and analysed using Stereo Investigator software (MBF Bioscience, Williston, Vermont, USA) and Olympus Fluoview FV10-ASW (Olympus Corporation, Tokyo, Japan). Z-stacks, three-dimensional, and orthogonal views were generated in Volocity High Performance 3D Imaging Software. All image manipulations were limited to brightness/contrast, and were performed in a standardized manner for all images. Images were assembled into figures using Adobe Illustrator (Adobe Systems, Inc., San Jose, $\mathrm{CA})$.

Semi-quantitative evaluation of grafted spinal cord tissue infiltration with different types of leukocytes in animals immunosuppressed using TAC biodegradable 3-month lasting pellets was performed using 40- $\mu$ m-thick coronal sections. Quantification was performed in 5 sections per animal in TAC dose groups of $1.8 \mathrm{mg} / \mathrm{kg} /$ day and $5.1 \mathrm{mg} / \mathrm{kg} /$ day (see Table VIII-1, Groups No. 7 and No. 9; $n=3$ for each group).

The number of different classes of leukocytes positive for CD45, CD8/CD45, CD4/CD45, RT1B/CD45 and CD11B were counted and analyzed separately with respect to the location of positive cells in: 1) the core of the graft; 2) the pial surface of the graft, and 3) the spinal cord parenchyma outside of the graft. The degree of leukocyte infiltration was graded as follows: 0 - absence of immune cells, *1-10 cells, ${ }^{* *}$ - $11-50$ cells, ${ }^{* * *}>51$ cells per section.

\section{Quantitative analysis of grafted cell survival}

Quantitative analysis of grafted cell survival was performed in animals immunosuppressed with 1.8 $\mathrm{mg} / \mathrm{kg} /$ day of TAC or $5.1 \mathrm{mg} / \mathrm{kg} /$ day of TAC (TAC pellet groups No. 7 and 9; see Table VIII-1 \& 2) and grafted with HUES7-NSCs or hSCC ( $n=2$ for each TAC dose and cell line).

For quantification, 5 transverse spinal cord sections were selected (minimum $500 \mu \mathrm{m}$ apart) from the cell-grafted segments from each rat. An image of virtual tissue of the whole hNUMA-stained section was captured at 10X using Olympus Imager M2 microscope equipped with MBF Stereo Investigator System. The total number of NUMA+ nuclei was then counted using ImageJ "Analyze Particles" plug-in function after setting an identical detection threshold for all analyzed sections. The number of counted cells was then averaged and expressed as number of cells counted per section for each TAC dose and grafted cell line analyzed.

All images used for quantification (i.e., leukocyte-stained and hNUMA+ stained sections) were examined by a blinded observer. 


\section{Flow cytometry}

Peripheral blood from rats (saphenous vein) was collected into microtainer EDTA tubes (BD Biosciences, San Jose, CA, USA). Red bloods cells were lysed using RBC lysis buffer (Sigma-Aldrich, St. Louis, MO, USA). The lysed blood was stained with antibodies directed to rat CD4, CD8, CD45, and CD3 (Biosciences, San Diego, CA, USA). A minimum of 10,000 live events (defined by FSC and 7-AAD) was acquired on a six-color BD FACSC. Analysis was done using FACSDiva software. CD4+ and CD8+ cell percentages was measured using a $T$ cell lineage gate defined by 7AAD- CD45+ CD3+ small lymphocytic cells.

\section{Results}

\section{Pharmacokinetic profile after subcutaneous TAC delivery (Fig. VIII-1)}

Four different TAC vehicle-delivery systems were used for subcutaneous delivery (see Table VIII-1 for details). 1) TAC-containing caster oil/saline solution: the whole blood TAC concentration in animals receiving a single bolus of TAC ( $3 \mathrm{mg} / \mathrm{kg}$ ) was on average $39.5 \mathrm{ng} / \mathrm{ml}$ at $9 \mathrm{hrs}$ after injection and decreased to $9 \mathrm{ng} / \mathrm{ml}$ at $24 \mathrm{hrs}$. With continuing daily injections at the same dose (i.e., $3 \mathrm{mg} / \mathrm{kg}$ ), the plasma concentration of TAC was 12.2 and $7.2 \mathrm{ng} / \mathrm{ml}$ at 72 and $120 \mathrm{hrs}$, respectively (i.e., at 24 hrs after the previous injection) (Fig. VIII-1A); the whole blood TAC concentration in animals receiving a single bolus of TAC (1.5 mg/kg/BID) was $11.9 \mathrm{ng} / \mathrm{ml}$ at $12 \mathrm{hrs}$ after injection. With continuing injections every $12 \mathrm{hrs}$ at the same dose (i.e., $1.5 \mathrm{mg} / \mathrm{kg}$ ), the blood concentration of TAC was on average 13.5 and $14.9 \mathrm{ng} / \mathrm{ml}$ at 24 and $120 \mathrm{hrs}$, respectively (i.e., at $12 \mathrm{hrs}$ after the previous injection delivered at $12 \mathrm{hrs}$ and $108 \mathrm{hrs}$, respectively) (Fig. VIII-1B). 2) TAC-containing unilamellar or multilamellar liposomes: the blood TAC concentration in animals receiving a single bolus of unilamellar or multilamellar liposomes-containing TAC (3 mg/kg) was on average 52.9 (unilamellar liposomes) and $52 \mathrm{ng} / \mathrm{ml}$ (multilamellar liposomes) at $12 \mathrm{hrs}$ and decreased to 6.1 (unilamellar) and $3.6 \mathrm{ng} / \mathrm{ml}$ (multilamellar) at $72 \mathrm{hrs}$, respectively (Fig. VIII-1C). 3) TAC-containing microspheres: the whole blood TAC concentration in animals receiving a single bolus of TAC-containing microspheres (20 mg/kg or $10 \mathrm{mg} / \mathrm{kg}$ ) showed a biphasic TAC release profile. The first peak was measured at 4 days after delivery and was 19.6 (20 mg/kg dose) and $6.4 \mathrm{ng} / \mathrm{ml}$ (10 mg/kg dose), respectively. The second peak was measured at 12 days after delivery and was $12.6(20 \mathrm{mg} / \mathrm{kg}$ dose) and $8.3 \mathrm{ng} / \mathrm{ml}(10$ $\mathrm{mg} / \mathrm{kg}$ dose), respectively. The TAC concentration at 19 days after delivery was on average 1.5 and $1.7 \mathrm{ng} / \mathrm{ml}$ in $20 \mathrm{mg} / \mathrm{kg}$ and $10 \mathrm{mg} / \mathrm{kg}$ group, respectively (Fig. VIII-1D). 4) TAC-containing pellets: groups of animals were implanted with 3-month releasable TAC pellets releasing TAC at 1.9, 3.4 or $5.1 \mathrm{mg} / \mathrm{kg} /$ day. In the $1.9 \mathrm{mg} / \mathrm{kg}$ group $(\mathrm{n}=4)$ the peak value of $11.1 \mathrm{ng} / \mathrm{ml}$ was measured 13 days after pellet implantation and then gradually decreased to $2.8 \mathrm{ng} / \mathrm{ml}$ at 90 days. In the $3.4 \mathrm{mg} / \mathrm{kg}$ 

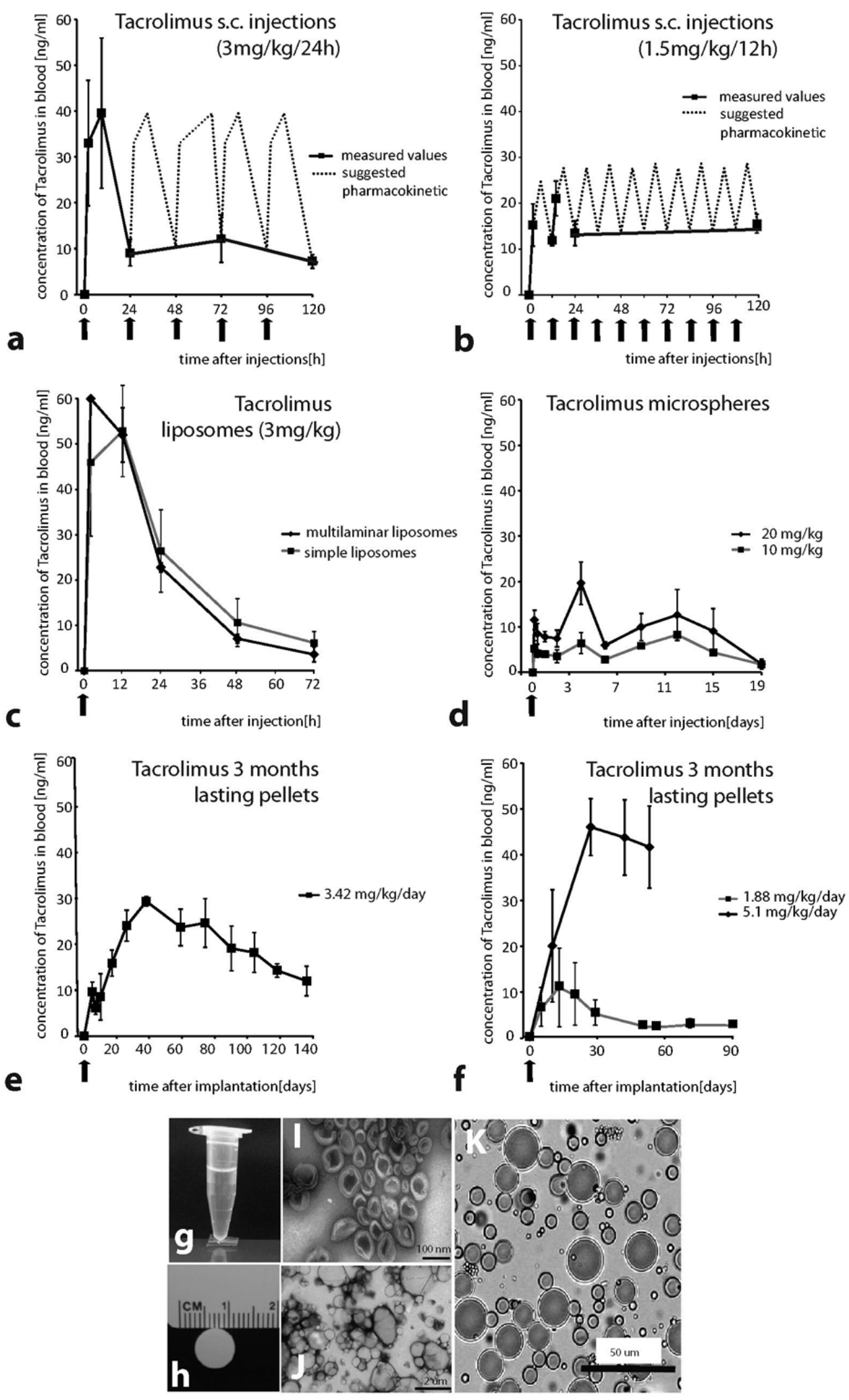

Figure VIII-1. Blood TAC pharmacokinetics after subcutaneous injections of four different TAC formulations. TAC was injected every $24 \mathrm{hrs}$ for 5 days ( $3 \mathrm{mg} / \mathrm{kg}$ ) (a) or every $12 \mathrm{hrs}$ for 5 days (1.5 mg/kg/12 hrs) (b). Blood was collected prior to subsequent TAC injection (arrows). Second, TAC loaded unilamellar or multilamellar (c) liposomes were injected subcutaneously as a single bolus (arrow) (3 mg/kg) and TAC concentration measured periodically for $72 \mathrm{hrs}$. Third, another 
two groups of animals were injected with single bolus of biodegradable TAC microspheres in the dose $10 \mathrm{mg} / \mathrm{kg}$ or $20 \mathrm{mg} / \mathrm{kg}$ (d). Note the lack of TAC plasma concentration "overshoot" during the first 9 hrs after delivery and a relatively stable plasma TAC concentration for up to 15 days after a single TAC microspheres bolus injection. Fourth, the biodegradable 3-month releasable TAC pellets delivering $1.88 \mathrm{mg} / \mathrm{kg} /$ day, $3.42 \mathrm{mg} / \mathrm{kg} /$ day or $5.1 \mathrm{mg} / \mathrm{kg} /$ day of TAC were implanted s.c. and the TAC plasma measured for up to 4.5 months $(e, f)$. Note a progressive increase in TAC concentration during the initial 3-4 weeks after TAC pellet implantation and then followed by a relatively stable and dose-dependent long-lasting TAC release. Arrows indicate the TAC pellet implantation. Each time point is represented as the mean \pm SD of TAC concentrations. Macroscopical and microscopical images of different TAC formulations including caster oil-saline (g), $90 \mathrm{mg}$ pellet (h), unilamellar (i) or multilamellar (j) liposomes or microspheres (k).

group $(n=3)$ the peak TAC concentration was measured at 38 days after pellet implantation and was on average $29.4 \mathrm{ng} / \mathrm{ml}$. At 3 months the TAC levels were $19.1 \mathrm{ng} / \mathrm{ml}$. In the $5.1 \mathrm{mg} / \mathrm{kg}$ group ( $\mathrm{n}=6$ ) the peak of TAC concentration was detected at 27 days and was $46 \mathrm{ng} / \mathrm{ml}$. At 53 days the TAC concentration was $41.7 \mathrm{ng} / \mathrm{ml}$ (Fig. VIII-1E, F). Macroscopical and microscopical images of all TAC formulations used in this study including caster oil-saline, $90 \mathrm{mg}$ pellet, unilamellar or multilamellar liposomes and microspheres is shown in Fig. VIII-1G-K.

\section{Tolerability of SD rats to long-term TAC pellet-induced immunosuppression}

Animals implanted with 3-month releasable pellets (dose $1.9 \mathrm{mg} / \mathrm{kg} /$ day) showed good tolerability for up to 3 months after pellet implantation and no detectable clinically-defined side effects were noted.

In the animal group implanted with $3.4 \mathrm{mg} / \mathrm{kg} /$ day TAC pellets, good tolerability was seen for up to 3 months without any prophylactic antibiotic treatment. Three animals were allowed to survive for an additional 2 months while a progressively decreasing TAC blood concentration was monitored. At the end of 5 months, 2 animals succumbed to infection, which was determined to be of gastrointestinal origin.

In the animal group receiving the highest TAC dose $(5.1 \mathrm{mg} / \mathrm{kg} /$ day), good tolerability was seen for up to 6-7 weeks after pellet implantation. From the total number of 60 animals so far tested in our laboratory with this dose, 3 animals showed nephrotoxicity at 7-8 weeks. Clinically, these animals showed increased agitation and tactile allodynia. In all 3 animals plasma TAC levels were higher than $60 \mathrm{ng} / \mathrm{ml}$. Once these symptoms were identified, the animals usually died within 2-3 days. Postmortem necropsy showed clear kidney atrophy and the presence of blood in the urine (i.e., hematuria). An additional 4 animals were found dead without any pre-clinical signs of toxicity 
between 3-8 weeks after pellet implantation. In 6 of 44 implanted animals, a cyst partially filled with serous fluid and surrounding the pellet implanted-subcutaneous region was identified.
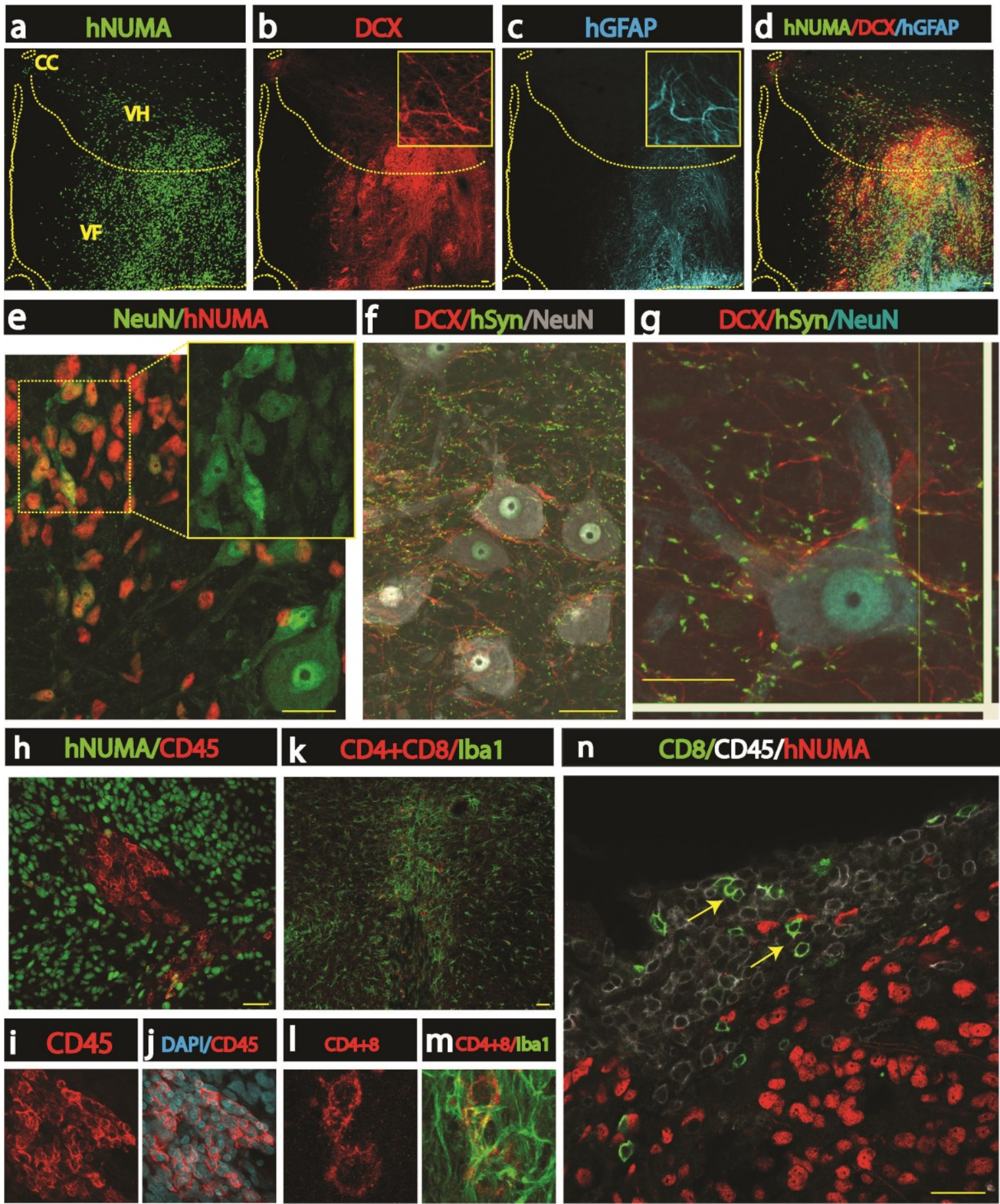

Figure VIII-2: Immunofluorescence examination of the presence of grafted human ES-derived neural precursors (HUES7-NPC) and immunological response in the spinal cord tissue of SOD+ rats implanted with 3-month lasting TAC pellet $(1.9 \mathrm{mg} / \mathrm{kg} / \mathrm{day})$. TAC releasable pellets were implanted subcutaneously in SOD+ rats ( $n=4$; 60-65 days of age) 13 days prior to grafting with HUES7-NPC. After cell grafting, animals were allowed to survive until they reached the endstage of the ALS disease (approx. 77 days after cell implantation). Immunohistochemical staining with human specific antibodies (hNUMA-human nuclear 
protein, green; hGFAP-human astrocytes, cyan) and doublecortin (DCX, red) revealed consistent presence of well-engrafted cell populations in the spinal parenchyma (a-d). Colocalization of hNUMA (red) with NeuN (green) can be seen in a dense population of hNUMA+ grafted cells (e). Triple labeling with DCX, hSYN and NeuN antibody showed numerous DCX+ processes projecting towards large $\alpha$-motoneurons and coexpressing hSYN punctate-like immunoreactivity $(f, g)$. The immunosuppressive effect of a given TAC dose $(1.9 \mathrm{mg} / \mathrm{kg} /$ day $)$ was further tested by identifying the presence of infiltrating T-lymphocytes. A high density leukocyte population (CD45, red) in the vicinity of grafted human cells (hNUMA, green) was observed (h-j). Co-staining with markers of T-lymphocytes (CD4 and CD8, red) and microglia (IB1, green) revealed the presence of T-lymphocytes and activated microglia in the areas of grafted cells $(\mathrm{k}-\mathrm{m})$. The presence of cytotoxic subpopulation of T-lymphocytes in the regions containing hNUMA+ grafted cells (red) was confirmed by colocalization of marker CD8 (green) with general leukocyte marker CD45 (white), (n). VH ventral horn, VF - ventral funiculus. Scale bar a-n: $30 \mu \mathrm{m}$.

Survival and maturation of spinally grafted human fetal spinal cord stem cells (hSSC) or human ES-derived neural precursors (HUES7-NPC) in SOD+ rats and TAC-dose dependent suppression of T-cell infiltration (Figs. VIII-2 \& 3).

To validate the effectiveness of TAC pellet-induced immunosuppression in an experimental xenograft design, transgenic SOD+ rats were implanted with TAC pellets and then received spinal grafts of hSSC or HUES7-NPC 13 days after TAC pellet implantation. Based on the initial pharmacokinetic study, 3 groups of transgenic SOD+ rats previously implanted with TAC pellets delivering either $1.9 \mathrm{mg} / \mathrm{kg} /$ day, $3.4 \mathrm{mg} / \mathrm{kg} /$ day or $5.1 \mathrm{mg} / \mathrm{kg} /$ day of TAC were used for cell grafting. In addition, a group of SD rats with previous L3-4 compression injury were used. Spinal trauma animals were grafted with HUES7-NPC. Animals in all experimental groups survived between 32-105 days after cell grafting (see Table VIII-2 for experimental groups). In general, independent of the TAC dose group and grafted cell line, comparable long-term engraftment and maturation of grafted cells was seen in SOD+ animals.

In the $1.9 \mathrm{mg} / \mathrm{kg} /$ day TAC group (SOD+ rats) grafted with HUES7-NPC, triple-staining with humanspecific nuclear marker (hNUMA), early postmitotic neuronal marker DCX and human-specific GFAP antibody (hGFAP) of transverse spinal cord sections taken from grafted spinal segments showed well engrafted hNUMA+ cell populations composed of DCX+ neurons and hGFAP astrocytes at 77 days after grafting (Fig. VIII-2A-D). Double-staining with NeuN (neuronal nuclei antigen) and hNUMA showed that the majority of hNUMA+ cells in the gray matter acquired NeuN immunoreactivity (Fig. VIII-2E; insert). Confocal analysis of DCX/hSYN (synaptophysin)/NeuN-stained sections showed a dense population of hSyn+ terminals residing in the vicinity of ventral $\alpha$-motoneurons and were associated with DCX+ processes (Fig. VIII-2F, G). 

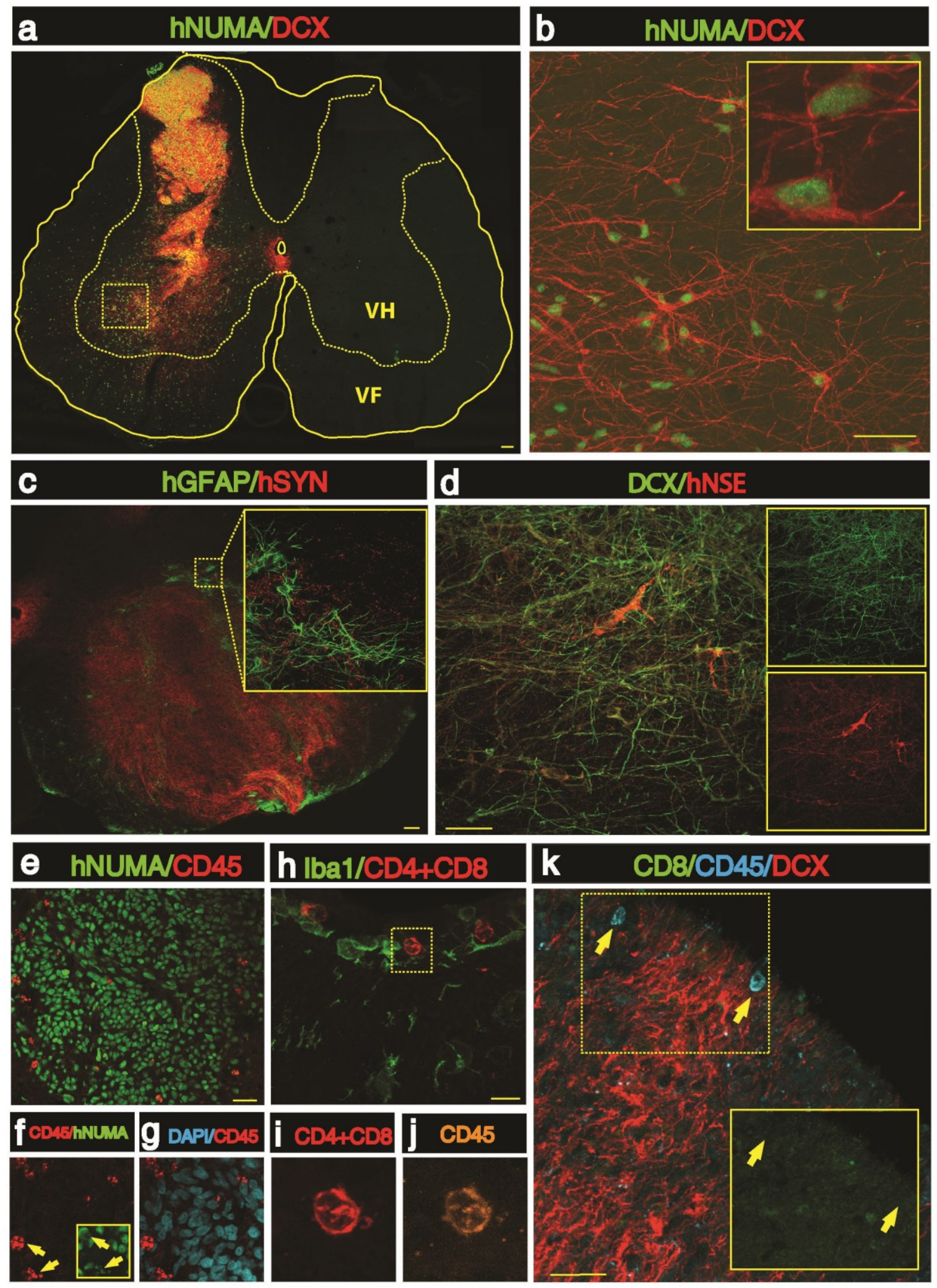

Figure VIII-3: Immunohistochemical examination of the presence of grafted human fetal spinal stem cells (hSSC) and immunological response in the spinal cord tissue of SOD+ rats immmunosuppressed with 3 month lasting TAC pellet $(5.1 \mathrm{mg} / \mathrm{kg} / \mathrm{day})$. TAC releasable pellets were implanted subcutaneously in the SOD+ rats ( $n=6 ; 60-65$ days of age) 13 days prior to grafting with hSSC. Animals were allowed to survive until $10 \%$ of bodyweight loss caused by the progression of the ALS disease (i.e., 32-70 days after cell 
implantation). Immunohistochemical staining with human specific antibody hNUMA (human nuclear protein, green) and doublecortin (DCX, red) revealed consistent presence of high density grafts in the targeted spinal parenchyma in all animals $(a, b)$. Double staining with human-specific synaptophysin (hSYN) and GFAP antibody (hGFAP) revealed a dense hSYN punctuate-like immunoreactivity and numerous hGFAP+ grafted astrocytes (c). In addition to a dense population of DCX+ grafted neurons the presence of humanspecific enolase+ neurons (hNSE; late neuronal marker) can be seen (d). The immunosuppressive effect of the administered dose of TAC $(5.1 \mathrm{mg} / \mathrm{kg} / \mathrm{day})$ was further validated by identifying the presence of Tlymphocytes in human cell-grafted spinal cord sections. Only occasional presence of CD45+ (red) leukocytes was seen; CD45+ elements can preferentially be seen at the periphery of hNUMA+ grafts (e-g). Co-staining with CD45 and CD8 antibody shows near complete lack of CD8+ immunoreactivity in CD45+ cells in cellgrafted spinal cord regions and only occasional double stained CD4/CD8/CD45+ cells can be identified (hj), while the majority of CD45+ cells are CD8 negative ( $k$; yellow arrows). VH - ventral horn, VF - ventral funiculus. Scale bar a,c: $100 \mu \mathrm{m}, \mathrm{b}, \mathrm{d}, \mathrm{e}, \mathrm{k}: 30 \mu \mathrm{m}, \mathrm{h}: 10 \mu \mathrm{m}$.

The degree of T-cell infiltration was probed by staining with CD45, CD4 and CD8 antibodies (see Table VIII-3 for quantitative analysis). High-density CD45+ cell populations were identified typically at the core or at the periphery of cell-grafted regions (Fig. VIII-2H-J). In the same areas CD4/8+ lymphocytes surrounded by activated IB1 immunoreactive microglial cells were seen (Fig. VIII-2K-M). Double-staining with CD45 and CD8 antibody showed a clear population of CD45/CD8+ cells in the vicinity of hNUMA+ grafted cells (Fig. VIII-2N; yellow arrows).

In the $5.1 \mathrm{mg} / \mathrm{kg} /$ day TAC group (SOD+ rats) grafted with hSSC, double-staining with hNUMA and DCX antibody showed a robust cell engraftment with high population of hNUMA/DCX+ grafted neurons throughout the grafted region and extending from the dorsal horn to ventral gray matter at 70 days after cell grafting (Fig. VIII-3A). Numerous solitary hNUMA/DCX+ neurons which migrated from the core of the graft were also identified (Fig. VIII-3B; inserts). Staining with human specific synaptophysin antibody showed a wide-spread hSYN punctate-like staining pattern throughout the grafted region (Fig. VIII-3C). In the same areas a high density of hGFAP+ astrocytes were also seen (Fig. VIII-3C-insert). Double staining with DCX and human-specific NSE antibody (hNSE) showed that in areas with high density of DCX+ processes solitary hNSE+ neurons were also present (Fig. VIII-3D).

Quantitative analysis of hNUMA+ cells in $1.9 \mathrm{mg} / \mathrm{kg} /$ day TAC group showed on average $2712 \pm 320$ cells in HUES7-NPC-grafted animals $(n=2)$ and $1772 \pm 629$ cells in hSSC-grafted group ( $n=2)$. In the 5.1 $\mathrm{mg} / \mathrm{kg} /$ day TAC group on average $2750 \pm 527$ cells were counted in HUES7-NPC-grafted animals $(n=2)$ and $2164 \pm 638$ cells were counted in the hSSC-grafted group $(n=2)$. 

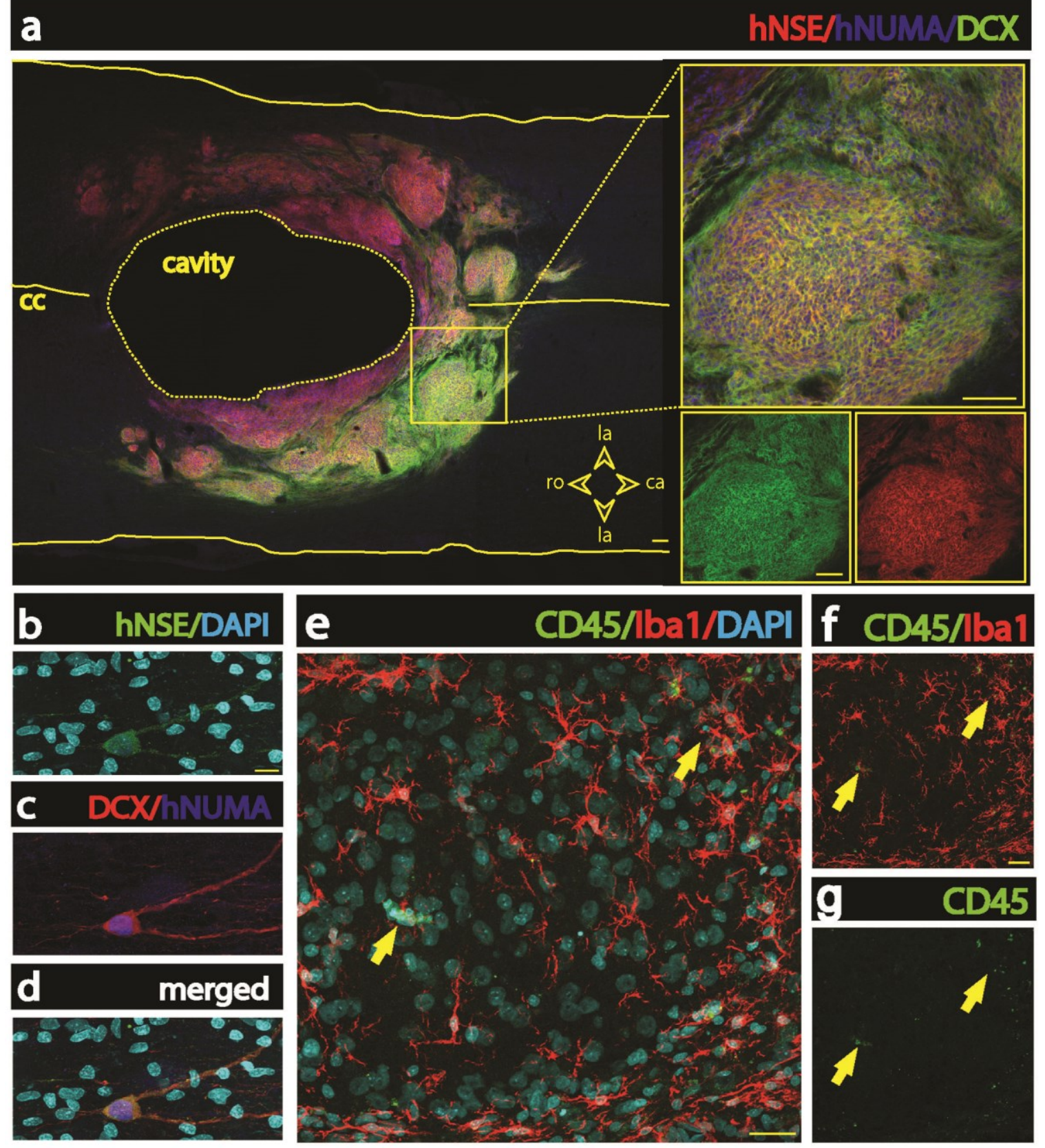

Figure VIII-4: Immunofluorescence examination of the presence of grafted human ES-derived neural precursors (HUES7-NPC) in TAC pellet $(4.9 \mathrm{mg} / \mathrm{kg} /$ day)-immunosuppressed SD rats with previous L3 contusion injury. Animals $(n=4)$ received spinal grafts of HUES7-NPC at 3 days after spinal injury and were allowed to survive 105 days after the cell grafting. Immunohistochemical staining with antibodies against human neuronal specific enolase (hNSE, red), human cell nuclei (hNUMA, blue) and doublecortin (DCX, red) on the longitudinally-cut spinal sections revealed continuous graft survival and extensive neuronal differentiation in cell-grafted peri-injury regions (a-d). Staining against the geneal leukocyte marker (CD45, green) and microglia (IB1, red) revealed the absence of leukocytes and the presence of necrotic bodies (nonspecific staining, green, yellow arrows) associated with the cytoplasm of microglial cells (e-g). cc = central canal, la = lateral, ro = rostral, ca = caudal. Scalebar a: $100 \mu \mathrm{m} ; \mathrm{b}-\mathrm{d}: 10 \mu \mathrm{m} ; \mathrm{e}, \mathrm{f:} \mathbf{3 0} \mu \mathrm{m}$. 
In both TAC dose groups ( 3.4 and $5.1 \mathrm{mg} / \mathrm{kg} /$ day), staining with CD45, CD4 and CD8 markers showed a similar pattern. The number of CD45+ stained cells was relatively low (if compared to 1.9 $\mathrm{mg} / \mathrm{kg} /$ day TAC group; see Table VIII-3 for quantitative analysis) with the majority of CD45+ cells typically found at the periphery of individual grafts (Fig. VIII-3E-G). Staining with CD4/8 antibody showed only sporadic presence of CD4/8+ cells (Fig. VIII-3H-J) and the majority of CD45+ cells found in grafted DCX+ regions were CD8 negative (Fig. VIII-3K; yellow arrows).

\begin{tabular}{cc|ccc|ccc}
\hline \multirow{2}{*}{$\begin{array}{c}\text { immunological } \\
\text { profile }\end{array}$} & Dose of Tacrolimus & \multicolumn{3}{c}{$1.8 \mathrm{mg} / \mathrm{kg} / \mathrm{day}$} & \multicolumn{3}{c}{$5.1 \mathrm{mg} / \mathrm{kg} / \mathrm{day}(\# \#)$} \\
\cline { 2 - 8 } & cell type & G-core & G-pia & SC-par & G-core & G-pia & SC-par \\
\hline CD45+ & leukocytes & $* * *$ & $* * *$ & $* * *$ & $*$ & $* *$ & $*$ \\
CD8+CD45+ & cytotoxic T-cells & $* *$ & $* * *$ & $*$ & 0 & $*$ & 0 \\
CD4+CD45+ & helper T-cells & $* * *$ & $* * *$ & $* *$ & $*$ & $*$ & 0 \\
RT1B+CD45+ & B-lymphocytes & $* *$ & $* * *$ & $* *$ & $*$ & $*$ & 0 \\
CD11b+ & $\begin{array}{c}\text { granulocytes+ } \\
\text { monocytes }\end{array}$ & $* *$ & $* * *$ & $*$ & $*$ & $*$ & 0 \\
\hline
\end{tabular}

Tab.3 Quantitative/qualitative analysis of leukocyte infiltration in transverse spinal cord sections with identified human cell grafts at 77-90 days after cell grafting; $0=$ absence of immune cells, $*-1-10$ cells, $* *$ $11-50$ cells, $* * *>51$ cells per section; $\mathrm{G}=$ core- core of the graft, $\mathrm{G}=$ pial surface of the graft, SC-par = parenchyma of the spinal cord outside of the graft, \#\# = in animals injected with $3.4 \mathrm{mg} / \mathrm{kg} / \mathrm{day}$ Tac dose a comparable suppression of leukocyte infiltration as seen in $5.1 \mathrm{mg} / \mathrm{kg} /$ day group was identified (not shown).

Survival and maturation of spinally grafted HUES7-NPC in adult SD rats with previous lumbar (L3) spinal contusion injury (Fig. VIII-4)

To characterize the survival of spinally grafted human ES-derived neural precursors, adult SD rats with previous L3 spinal contusion injury received spinal grafts of HUES7-NPC at 3 days after injury and survived for 3 months. The dose of TAC delivered by implanted TAC pellet was $4.9 \mathrm{mg} / \mathrm{kg} / \mathrm{day}$. Immunofluorescence analysis of hNSE/hNUMA/DCX triple-stained sections showed an advanced stage of neuronal maturation as evidenced by an intense hNSE immunoreactivity (Fig. VIII-4A; inserts). Numerous double stained hNSE/DCX solitary neurons which migrated outside of the grafts were also identified (Fig. VIII-4B-D). Similarly as in SOD+ rats immunosuppressed with a high TAC dose, staining with CD45 antibody revealed only occasional presence of CD45+ cells at the periphery of individual grafts (Fig. VIII-4E-G). Co-staining with CD45 and CD8 antibody showed only occasional presence of CD8+ cells (not shown). 
circulating T-lymphocytes
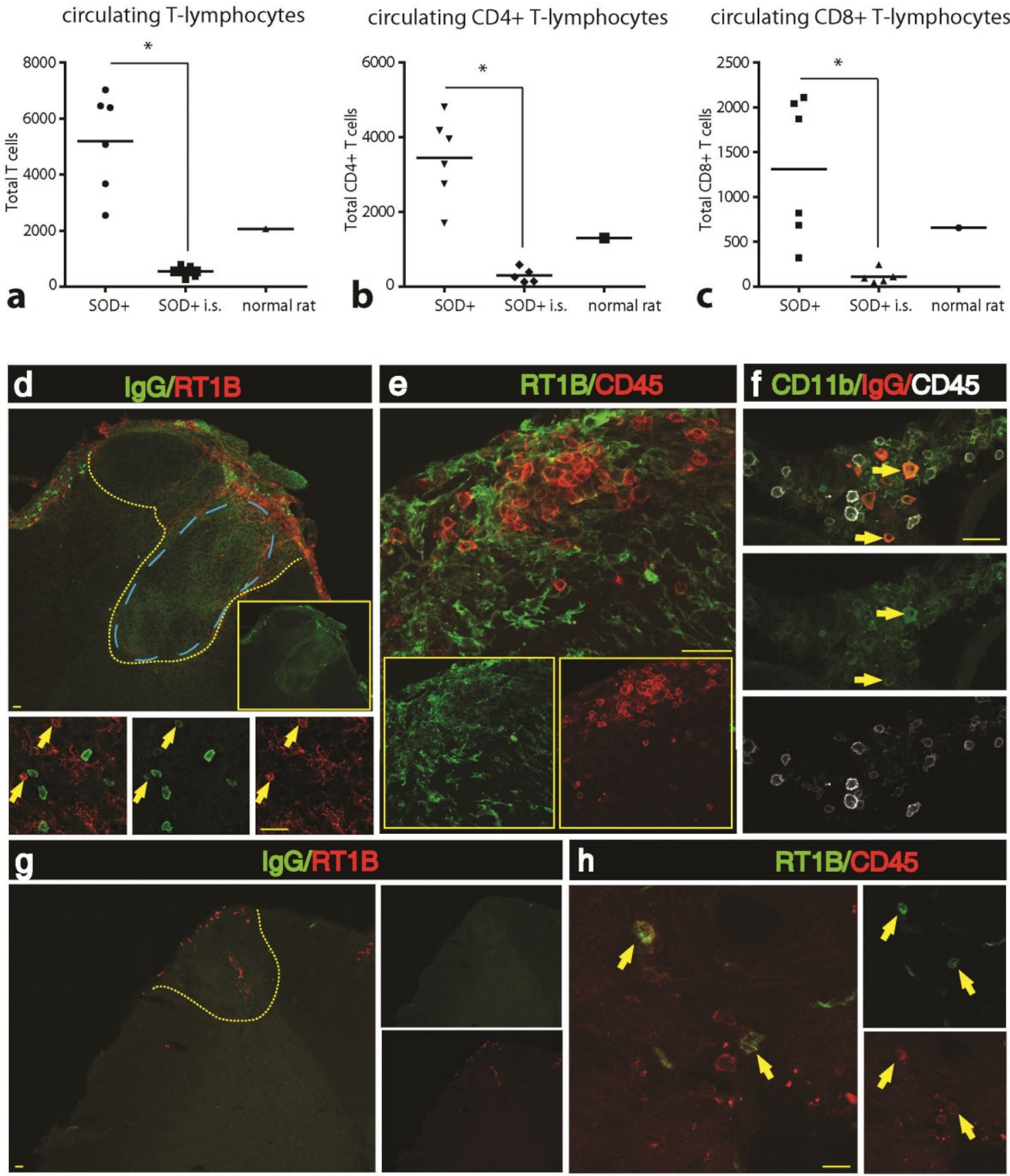

RT1B/CD45

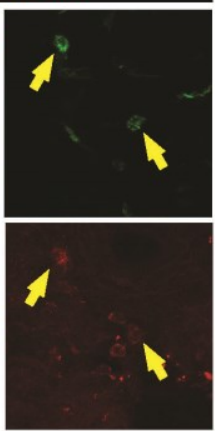

Figure VIII-5: Effect of TAC pellet-induced immunosuppression of circulating T-cells and the spinal infiltration with IgG+ cells, B-cells and granulocyte/monocyte in cell-grafted animals. In TAC pellet-immunosuppressed animals $(5.1 \mathrm{mg} / \mathrm{kg} /$ day), a significant suppression in the number of circulating blood CD4 and CD8 Tlymphocytes was measured using flow cytometry $(a-c),(* p<0.05 ;$ T-test). Staining with anti-rat IgG (fluorescence-tagged), anti MHC class II (RT1B) and with anti-CD45 antibody in the $1.9 \mathrm{mg} / \mathrm{kg} /$ day TAC group showed a high density of IgG+ cells at the periphery of grafted regions (d). In addition, an overall increase in the density of IgG staining in the same areas was seen (d; blue dashed area). Triple staining with CD11b, anti-rat IgG and CD45 antibody showed co-expression of CD11b in anti-rat IgG+ cells (f; yellow arrows) but no clear colocalization with CD45 marker was detected (f; lower panel). In $5.1 \mathrm{mg} / \mathrm{kg} / \mathrm{day}$ TAC group a near complete lack of anti-rat IgG, RT1B and CD45+ cell was seen (g) and only sporadic occurrence of RT1B/CD45+ cells was seen at the periphery of grafted regions (h; yellow arrows). 


\section{Effect of TAC pellet-induced immunosuppression on circulating blood T-cell population and}

IgG immunoreactivity in spinal human cell-grafted regions (Fig. VIII-5)

To further probe the effect of TAC pellet-induced immunosuppression on T-cell activity, we quantified the density of circulating T-cell population in blood in naïve, SOD+ TAC non-treated and SOD+ TAC-treated $(5.1 \mathrm{mg} / \mathrm{kg} /$ day) animals. In comparison to TAC non-treated animals, immunosuppressed rats had a significantly decreased number of circulating CD45, CD4 and CD8 cells (Fig. VIII-5A-C), ( $p<0.05$; t-test).

To identify the presence of IgG-secreting cells or soluble IgG in cell-grafted spinal cord regions, sections taken from all 3 TAC dosing groups were stained with anti-rat IgG (fluorescence-tagged) and then further stained to detect MHC class II (R1TB) and CD45 to identify B cells. In $1.9 \mathrm{mg} / \mathrm{kg} /$ day TAC group numerous IgG+ cells were identified in grafted regions. Similarly as for the CD8 population, the IgG+ cells were typically localized at the periphery of individual grafts. Double staining with anti-rat MHC class II antibody and anti-rat IgG showed the B-lymphocyte phenotype in small subpopulation of IgG+ cells (Fig. VIII-5D; yellow arrows). In addition to IgG presence on B-lymphocytes, an overall increase in the density of IgG staining in cell-grafted regions was seen (Fig. VIII-5D; blue dashed area). Since the area had minimal R1TB staining, it is possible that deposited IgG is detected in this area. Similarly, double staining with RT1B and CD45 antibody showed only sporadic presence of RT1B/CD45-double-stained cells in cell-grafted areas (Fig. VIII-5E).

Triple staining with macrophage and monocyte marker CD11b, anti-rat IgG and CD45 showed a clear colocalization of CD11b and anti-rat IgG immunoreactivity but with relatively weak CD45 staining pattern in the same CD11b/anti-rat IgG+ cells (Fig. VIII-5F; yellow arrows).

In 3.4 and $5.1 \mathrm{mg} / \mathrm{kg} /$ day TAC groups, only sporadic IgG, RT1B or CD45-stained cells were identified as well as the density of the IgG staining in cell-grafted regions was below detection threshold (Fig. VIII-5G, H) and was similar to that seen in control cell-non-grafted animals (not shown). 
Rejection of spinally grafted human fetal spinal cord stem cells (hSSC) in the absence of immunosuppression (Fig. VIII-6)

To validate the importance of immunosuppression in providing grafted human cell survival, we next grafted hSSC into lumbar spinal cord in adult SD rats without immunosuppression. Analysis of spinal cord sections at 14 days after cell grafting showed complete grafted cell rejection and intense infiltration of CD4/8 cells at the injection site (Fig. VIII-6 A, B, C, D).
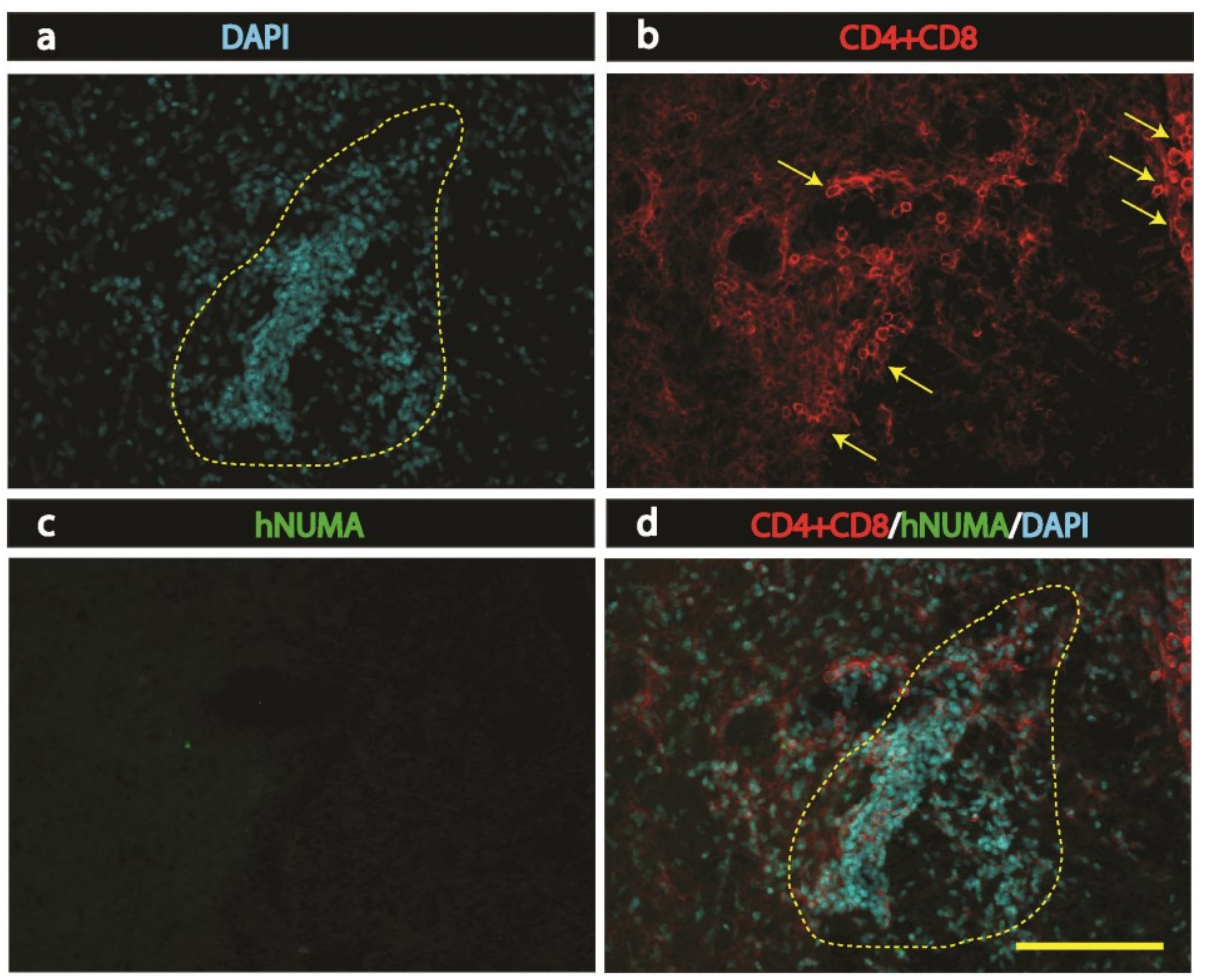

Figure VIII-6: Rejection of spinally grafted hSSC in SD rats in the absence of immunosuppression. Adult SD rats received 10 bilateral injections of hSSC into lumbar spinal cord and survived for 14 days. No immunosuppression treatment was used in any animal. Triple staining of transverse spinal cord sections with DAPI and hNUMA and CD4/8 antibody showed increased cellularity in cell grafted region (a; yellow dashed circle) but with no grafted cell survival as evidence by the lack of hNUMA+ cells (c). Instead an intense accumulation of CD4/8 cells in previously cell-grafted regions can be seen (b, d; yellow arrows). Scale bar a: $100 \mu \mathrm{m}$. 


\section{Discussion}

Experimental and clinical evidence for need of continuing immunosuppression in xenogeneic CNS grafting design

Extensive previous experimental animal but also clinical data show that xenogeneic neural grafts are rapidly rejected in non-immunosuppressed animals and/or human patients. For example, it was demonstrated that grafted porcine dopaminergic neuroblasts (derived from embryonic day 26-27) are rejected within days to weeks after intracerebral grafting in rats in the absence of immunosuppression (Brevig 2000). It was also demonstrated that the porcine xenografts undergoing rejections are infiltrated with CD8 lymphocytes and are stained positive for IgM and complement component (C3) (Barker 2000). Similarly, in clinical Parkinson's disease trial, rafting of porcine embryonic dopaminergic neurons showed very poor grafted cell survival at 7 months (one postmortem patient analyzed) after grafting despite continuing immunosuppression with cyclosporine (5 mg/kg) (Deacon 1997). In the same study, lymphocyte infiltration was also seen in the cell-grafted region. These data are consistent with our previous reports which demonstrate no or poor graft survival after spinal grafting of human spinal stem cells in SOD+ rats or SD rats with previous spinal ischemic injury if animals were immunosuppressed with TAC as a monotherapy (1.5 mg/kg, q12h or $1 \mathrm{mg} / \mathrm{kg}$, qd, respectively) (Hefferan 2011, Kakinohana 2012). Similarly, grafted cell rejection was associated with an intense CD4/8 T-lymphocyte infiltration in previously cell-grafted regions. Jointly, these data show that in order to achieve satisfactory and long-term grafted cell survival using xenogeneic grafting design, continuing and aggressive immunosuppression which is effective in suppressing T-cell activity is needed.

Stable and controllable blood TAC concentration and tolerability of SD rats to long-term TAC pellet-induced immunosuppression

In our initial phase of the study, we have compared the plasma kinetics of four different TAC formulations after subcutaneous delivery. Three primary characteristics of the PK data and associated animal manipulations (such as repetitive animal injections) were considered in defining the optimal formulation to be used in the subsequent long-term spinal cell grafting studies and included: 1) the stability of plasma TAC concentration over a 24-hr period, 2) required frequency of injections to achieve a targeted plasma TAC concentration, and 3) potential side effects associated with a high plasma TAC concentration or repetitive animal injections.

The rationale for the selection of specific time points for TAC measurements (i.e., more frequent measurements in caster oil/saline, liposomes and microspheres formulations-injected animals and less frequent in TAC pellet-implanted animals; see Table VIII-1 for summary) was based on expected 
differences in TAC kinetics among all formulation and by the need to identify potential blood toxic TAC levels.

First, in caster oil-saline as a vehicle or liposome (unilamellar or multilamellar) TAC-injected animals, a comparable kinetics profile was seen during the initial $12 \mathrm{hrs}$ after $3 \mathrm{mg} / \mathrm{kg}$ TAC injections with the peak concentrations $(40-60 \mathrm{ng} / \mathrm{ml})$ measured during 2-12 $\mathrm{hrs}$ after injections. In contrast to the caster-oil-saline TAC ( $3 \mathrm{mg} / \mathrm{kg}$ ) group in which the TAC levels dropped to $10 \mathrm{ng} / \mathrm{ml}$ at $24 \mathrm{hrs}$ after single injection, in liposome-TAC injected animals $(3 \mathrm{mg} / \mathrm{kg}$ ) on average $27 \mathrm{ng} / \mathrm{ml}$ TAC was measured at $24 \mathrm{hrs}$ and was still around $11 \mathrm{ng} / \mathrm{ml}$ at $48 \mathrm{hrs}$ after injection. In animals injected BID with the caster-oil-saline formulation ( $1.5 \mathrm{mg} / \mathrm{kg} / 12 \mathrm{hrs}$ ) for 5 days, the measured peak TAC concentration was seen at $2 \mathrm{hrs}$ (around $23 \mathrm{ng} / \mathrm{ml}$ ) and stable levels around $13 \mathrm{ng} / \mathrm{ml}$ were then measured between 1-5 days if measured at the end of $12 \mathrm{hrs}$ (i.e., at $12 \mathrm{hrs}$ after previous TAC injection). These TAC pharmacokinetic data are similar as reported from other laboratories using mice or rats (Ko 1994, McAlister 1998, Yamauchi 2004).

From the perspective of conducting long-term immunosuppressive therapy, these data demonstrate that, should a caster-oil vehicle system be used for TAC delivery, the BID delivery regimen has the most favorable safety profile (as defined by the peak concentration) and is relatively stable through TAC levels. While more labor intensive, a stable targeted through TAC levels can be achieved and readily adjusted if needed. The use liposome-TAC formulation provides an extended half life, however, a relatively high peak concentration (i.e., above $50 \mathrm{ng} / \mathrm{ml}$ ) and the cost (approx. $\$ 1,600 / 6$ animals) to prepare these formulations appears to limit its routine use at present.

Second, TAC microspheres were used and animals received a single subcutaneous injection of either 10 or $20 \mathrm{mg} / \mathrm{kg}$ TAC-containing microspheres. In both doses, a biphasic release profile was measured with the first peak seen at 4 days and the second peak at 12 days after injection. In the $20 \mathrm{mg} / \mathrm{kg}$ group, the peak measured at 4 days was around $20 \mathrm{ng} / \mathrm{ml}$ and the levels were still around $10 \mathrm{ng} / \mathrm{ml}$ at 15 days after a single injection. This TAC kinetics profile is similar if not identical as reported in other studies which employed the same TAC microsphere formulation. In contrast to the caster-oil and liposome formulation, no initial TAC plasma concentration "overshoot" was seen at $2 \mathrm{hrs}$ after injection and levels were around $12 \mathrm{ng} / \mathrm{ml}$ at $9 \mathrm{hrs}$ after injection (in the $20 \mathrm{mg} / \mathrm{kg}$ group). The use of TAC microspheres appears to provide a clear advantage over caster-oil and liposome formulation, permitting stable TAC plasma levels for up to 15 days after a single subcutaneous injection. Moreover, additional injections can readily be added should a rapid and continuing stable increase in TAC concentration be desired. 
Third, TAC pellets containing different concentration of TAC and delivering $1.8 \mathrm{mg} / \mathrm{kg}, 3.4 \mathrm{mg} / \mathrm{kg}$ or $5.1 \mathrm{mg} / \mathrm{kg}$ of $\mathrm{TAC} /$ day for up to 3 months were used for subcutaneous implantation. In higher concentration groups, a progressive increase in plasma TAC concentration was seen during the initial 30 days after pellet implantation and then remained relatively stable for an additional 1-2 months. While the pellets are designed for 3-month continuing TAC release, we have observed continuing TAC release exceeding 4 months $(n=3)$ with the TAC levels around $13 \mathrm{ng} / \mathrm{ml}$ measured at 5 months after pellet implantation.

With respect to toxicity, no detectable side effects were seen in animals receiving 1.9 and 3.4 $\mathrm{mg} / \mathrm{kg} /$ day doses and surviving between 1-3 months. From a total of 60 animals receiving the 5.1 $\mathrm{mg} / \mathrm{kg} /$ day TAC dose, 4 animals died from apparent kidney toxicity at 1.5-2 months. These animals showed signs of agitation, tactile hypersensitivity as well as scratching behavior 3-4 days before death. We speculate that these behavioral signs could be the result of progressive kidney failure and increased creatine levels. Increased creatine levels have been reported in patients receiving higher dose of TAC after solid organ transplants (Finn 1999, Teh 2011).

Defining the optimal TAC immunosuppressive regimen to permit long-term survival and maturation of spinally grafted human neural precursors in rats

As demonstrated in our current study independent of the daily TAC dose delivered (i.e., 1.9, 3.4 or $5.4 \mathrm{mg} / \mathrm{kg} /$ day) in the form of a TAC pellet, consistent survival of grafted ES-derived NPC or human fetal spinal cord stem cells was seen at intervals 30-90 days after grafting. This was expressed as the presence of high density DCX or NSE immunoreactive-grafted neurons in targeted spinal cord regions. In addition, high density neuronal processes derived from grafted human neurons expressing human-specific synaptophysin and projecting toward host interneurons and $\alpha$ motoneurons were identified. Comparable grafted cell survival and maturation was seen in both SOD+ rats and in SD rats with previous L3 contusion injury. In our previous experiment, we demonstrated consistent xenograft survival three weeks after the transplantation of hSSC into the spinal cord in SOD+ rats treated with daily i.p. injection of TAC ( $3 \mathrm{mg} / \mathrm{kg} /$ day) combined with daily i.p. injection of Mycophenolate mofetil (30 mg/kg) (Hefferan 2011).

As shown in the present study, the SOD+ mutant rats show a significant increase in circulating CD4/8 cells if compared to naïve SD rats and this increase in CD4/8 T-lymphocytes was completely blocked by high dose (5.4 mg/kg/day) TAC treatment (Fig. VIII-5; a-c). In addition, several previous studies have demonstrated significant spinal inflammatory changes in symptomatic SOD+ rats characterized by intense activation of microglia and astrocytes in areas of previous $\alpha$-motoneuron loss (Hall 1998, Hefferan 2012). The characteristics of this model and requirement of continuous high dose TAC 
immunosuppression in order to provide long-term grafted cell survival further emphasize the importance of aggressive immunosuppressive therapy to achieve consistent cell survival in this neurodegenerative model.

Similarly, in our recent study, we demonstrated consistent cell survival for up to 2 months after spinal grafting of HUES7-NPC after using $1 \mathrm{mg}$ TAC/day dose delivered s.c. as a bolus in caster-oil preparation in SD rats with previous spinal ischemic injury. However, no cell survival was seen if animals were allowed to survive for total of 4 months (Kakinohana 2012). In another study, on average 28-day survival of allogeneic islet grafts in mice after single injection of TAC loaded microspheres (dose $20 \mathrm{mg} / \mathrm{kg}$ ) or continuing graft survival (>100 days) after repeated injections of TAC loaded microspheres (dose $10 \mathrm{mg} / \mathrm{kg}$; 7-day intervals) was described (Wang 2004). Jointly, these data show that the TAC dose of around $3 \mathrm{mg} / \mathrm{kg} /$ day and divided into two 12-hr doses (if delivered as a bolus s.c injection) or in a form of continuously TAC releasing pellet is required for long-term effective immunosuppression to permit xenograft survival in rats.

Translated to plasma TAC concentration, TAC levels of $\leq 15 \mathrm{ng} / \mathrm{ml}$ were measured in $1.5 \mathrm{mg} / \mathrm{kg} / 12 \mathrm{hr}$ (caster-oil formulation) or in 3.42 or $5.1 \mathrm{mg} / \mathrm{kg} / \mathrm{day}$ (pellet groups), i.e., the TAC dosing regimen which was associated with the most potent suppression of T-cell infiltration in the cell-grafted regions. Comparable TAC plasma levels were shown to be required to permit long-term survival of grafted pancreatic islet cells in mice (Wang 2004) or to lead to life-long survival of transplanted solid organs in human patients (Pirsch 1997, Przepiorka 1999, Staatz 2004, Vicari-Christensen 2009).

\section{TAC dose-dependent suppression of T- and B-lymphocyte activation and proliferation}

Quantitative analysis of T-cells (CD8, CD4) in grafted spinal cord regions showed a near complete absence of this cell population in 3.4 and $5.1 \mathrm{mg} / \mathrm{kg} /$ day TAC group if analyzed at 77-90 days after cell grafting. Similarly, the analysis of CD8 and CD4 cells in circulating blood showed a significant decrease in the $5.1 \mathrm{mg} / \mathrm{kg} /$ day TAC group if compared to non-immunosuppressed SOD+ rats. In contrast to the 3.4 and $5.1 \mathrm{mg} / \mathrm{kg} /$ day TAC groups, a clear population of CD4/8 cells was seen in 1.9 $\mathrm{mg} / \mathrm{kg} /$ day TAC-treated and cell grafted animals. Interestingly, a substantially higher density of CD8 population was seen on the dorsal surface of individual grafts facing the pial membrane. We speculate that the presence of grafted cells in heavily vascularized but BBB-lacking pial arterial system is more susceptible to extravasation of the circulating T-cell population and the resulting Tcell mediated response. We also hypothesize that even if a satisfactory cell survival was seen in the $1.9 \mathrm{mg} / \mathrm{kg} /$ day group at 77 days after cell grafting, a progressive grafted cell rejection will likely develop should the same level of low level immunosuppression continue over an extended period of time. We also saw small lymphocytic IgG+ MHC class II+CD45+ B cells in the graft surface. We 
speculate that these cells are releasing graft-specific antibodies that are bound the the graft or to CD11b+CD45- microglia cells.

\section{Limitation of subcutaneous TAC pellet-induced immunosuppression}

Several technical issues need to be considered once a pellet immunosuppression regimen is going to be implemented into any rat xenograft-immunosuppression study. 1) Based on our PK study, it takes on average 7-15 days after pellet implant before targeted ( $\leq 15 \mathrm{ng} / \mathrm{ml}$ TAC) plasma levels are achieved in 200-350 g rats. Thus, the TAC pellet needs to be implanted before cells are transplanted and the plasma level of TAC validated. However, because consistent and predictable plasma TAC concentrations were measured in our initial PK study in all 3 TAC pellet dosing groups, we have currently implemented less vigorous TAC plasma monitoring and the TAC concentration is only measured 1-2 times during the course of the 2-3 month post-pellet implantation period. Consistent with our initial PK TAC data, expected TAC concentrations were measured in more than 30 animals. 2) Should there be a desire to remove already implanted TAC pellets to achieve an abrupt termination of immunosuppression, the tissue surrounding the pellet implanted region needs to be excised in block. From our experience, the pellet identification is substantially obscured as soon as 714 days after implantation because of its structural disintegration. 3) Similarly, should there be a need to increase the dose of delivered TAC, an additional pellet(s) can be implanted. Should this be the case, TAC plasma monitoring is recommended to be performed in 5-7 day intervals for at least 23 weeks after additional pellet implant to identify potential unwanted toxic TAC concentrations. However, as shown in our current study, the plasma TAC concentrations up to $45-50 \mathrm{ng} / \mathrm{ml}$ were well tolerated for up to 3 months and only a fraction of animals displayed systemic side effects. We are currently testing the tolerability of repetitive TAC pellet implant $(3.5 \mathrm{mg} / \mathrm{kg} /$ day dose group) and have not seen any detectable side effects at 4.5 months of continuous immunosuppression at this dose (unpublished observation).

In conclusion, we demonstrated that by using an implantable 3-month lasting TAC-releasing pellet it is possible to achieve functionally effective immunosuppression in SD rats as defined by long-term survival and maturation of spinally grafted human neural precursors derived from human fetal spinal cord or from embryonic stem cell. Plasma TAC concentration of $\leq 15 \mathrm{ng} / \mathrm{ml}$ was found to be required to lead to a near complete suppression of T-cell activity in the human cell-grafted spinal cord region and in circulation blood and was readily achieved by 7-10 days after pellet implantation. Jointly, these data demonstrate that the use of implantable TAC pellets can represent an effective longlasting immunosuppressive drug delivery system which is technically simple to implement, is safe for 
an extended period of time (4.5 months) and is associated with consistent and long-term human neural precursor survival after grafting into the spinal cord of SOD+ or spinal trauma-injured SD rats. 


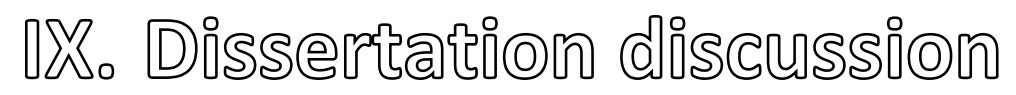

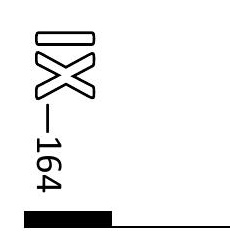




\section{Dissertation discussion}

In this chapter the results of our studies related to the research questions, as addressed in Chapter 1 , will be discussed. First, the reasons for the wide variance of pain prevalence in spinal cord injury (SCl) patient populations are explored (see below). Since most problems related to $\mathrm{SCl}$ still both have a chronic and therapy-resistant character, effective treatments are urgently needed (see below). Development of treatment strategies requires further fine-tuning of animal research for application in the clinical reality. Therefore the differentiation between spinal-reflex-mediated functionality and supra-spinally mediated functionality in rodent $\mathrm{SCl}$ is of great importance and needs first to be addressed (see below).

\section{Spinal Cord injury and its varying pain prevalence}

The first research question of this thesis was related to the large variance in reported $\mathrm{SCl}$ pain prevalence figures and therefore formulated as follows: "Is it possible to identify SCl population characteristics in published studies that can explain the differences in reported pain prevalence rates in SCl patients?". The answer to this research question can be subdivided into: A) there is a negative, and independent, relationship between reported pain prevalence rates and the study characteristics: 1) 'Strictness of the pain definition (mild, moderate or strict); more strict relates to lower pain prevalences', and 2) 'Whether or not a study was primarily a SCI pain study (yes vs. no); primary pain studies reported higher pain prevalences figures was noted, and B) a positive relationship for the population characteristics 'depression prevalence' and 'time interval of pain measurement after the initial SCl'; $\mathrm{SCl}$ populations with high depression rates and longer time intervals showed higher pain prevalences, but (in)dependence of the latter two variables could not be tested and is, indeed, possible (Chapter 2). Our meta-analysis was based on 82 studies (see Chapter 2), which reported on pain prevalence in SCl patient populations. To correct for the unwanted relationship between reported $\mathrm{SCl}$ pain prevalences and the study characteristics 'retrospective data', 'year of publication', and 'response rate', we excluded all retrospective studies, the studies published before 1960, and three studies which had reported both an extreme prevalence rate and a doubtful response rate (69 studies remained of the initial 82).

It should be noted that meta-analyses (of pooled data) do not prove any causal relationship, but only provide supporting evidence for such a correlation. A major complication in our, and most other, meta-analysis is the fact that correlations are based on regression analyses which might be suboptimal due to the use of aggregate/pooled patient data and not individual patient data.(Stroup 2000) It is easily understood that in case a study reports high pain prevalence and, at the same time, 
a high depression rate within its studied pooled patient population, it still is not possible to determine whether the individuals with pain and those with a depression are the same. An increase in the confidence on such correlations can be obtained through the inclusion of both a larger number of studies (with varying reported prevalence rates) and study samples in which all or most patients have a particular characteristic (e.g. a depression).

Hence, studies reporting on the pain prevalence of categorical subpopulations in their analysis, e.g. in patients all having a complete lesion, have also been included as such into our meta-analysis (for example: Anke et al. 1995 (Anke 1995)). Furthermore, our meta-analysis allows the study of categorical subpopulations reporting on pain prevalences of $\mathrm{SCl}$ patient which were either 1) having complete or incomplete injuries, 2) working or not working, 3) having traumatic or non-traumatic injuries, or 4) tetraplegic or paraplegic. However, these clinical patient characteristics did not result in any gain or loss of the already identified correlations with pain prevalence rates, making it unlikely that a marked relation between $\mathrm{SCl}$ pain and such clinical patient characteristics exists. However, with regard to the determinant 'depression' which did show a relation with pain prevalences; no studies were noted that report a subpopulation in which all individuals either report or do not suffer from a depression. Nonetheless, wide varieties in depression rates have been reported by the different studies, ranging from $2.2 \%$ up to $65.4 \%$ (Chapter 2).

Using categorical subpopulations one could also calculate relative risks for reporting pain for patients presenting a specific characteristic (compared to the ones not presenting that characteristic). Indeed, studies investigating pain predictors typically only report relative risks, not pain prevalences. These relative risks can also be used for a meta-analysis on pain determinants. Since our research question basically entails the identification of pain determinants, as any relationship between pain prevalence and a population characteristic is indicative for a pain determinant, one could argue that a meta-analysis of risk factors for $\mathrm{SCl}$ pain should have been more appropriate instead of a meta-analysis for $\mathrm{SCl}$ pain prevalence rates. We decided not to perform a meta-analysis of risk factors for $\mathrm{SCl}$ pain because the studies reporting relative risks for pain after $\mathrm{SCl}$ normally only do so for a limited amount of risk factors. Also, not many studies report risk factors, and the ones reported practically only involve patient-related determinants (and no methodological factors). Moreover, using risk factors no pain prevalence determinants have yet consistently been identified (Margot-Duclot 2009). Importantly, when using relative risks to determine factors influencing pain prevalence in a meta-analysis, one limits the possibility to analyze other pain determinants than the ones for which the relative risks are given (typically only a limited amount of patient-related determinants). Moreover, it is not unlikely that such other, underlying, determinants 
for pain prevalence might include methodological factors. The latter is particularly evident as our meta-analysis showed strong relationships between reported pain prevalences and methodological factors (e.g. use of retrospective data, difference in pain definition, and whether or not a study was a pain study).

By any means, from our meta-analysis on $\mathrm{SCl}$ pain prevalence rates we may conclude that pain is a major problem in the $\mathrm{SCl}$ population, as projected averages indicate that more than half of $\mathrm{SCl}$ patients has pain complaints.

\section{Spinal reflex mediated functionality and supra-spinally mediated functionality in rodent SCI models}

In order to explore the effectiveness of treatment approaches focused at the detrimental effects of $\mathrm{SCl}$, animal models are of great scientific value. However, investigating $\mathrm{SCl}$ treatments in animal models is controversial and has been the subject of an intense scientific debate since many years. For instance the subjectivity of pain in humans, let alone animals is something that is difficult, if not impossible, to catch in hard scientific data. Also (loco-) motor function differs significantly between humans and animals. An important underlying cause might be the difference in supraspinal ('conscious') CNS involvement for (sensi-)motor reflex function (Baastrup 2010b). This aspect is addressed in chapter $\mathbf{3}$ and $\mathbf{4}$ in order to answer our second research question: "Can we differentiate between spinal reflex mediated functionality and supra-spinally mediated functionality in rodent $\mathrm{SCl}$ models?".

With respect to sensory function we were able to demonstrate that while stimulus-evoked hindpaw withdrawal reflexes remained intact or even augmented after $\mathrm{SCl}$ (as we noticed decreased thresholds for paw withdrawal/jumping/kicking behavior), the evoked escape response paradigm revealed increased thresholds for below-level stimuli (chapter 3). Below-level paw withdrawal responses are thus poor outcome measures for pain/hypersensitivity tests in rodent SCl models, as they are mediated by spinal functional autonomy. Also, increased below-level as well as spinally mediated withdrawal reflexes did not coincide with muscle stretch hyperreflexia (as measured in the calf/gastrocnemius muscle; (Baastrup 2010a, Baastrup 2010b, Marsala 2005) and see chapter 3). This suggests that withdrawal reflexes do not correlate with spasticity, which then makes it more likely that paw withdrawal reflexes (after $\mathrm{SCl}$ ) relate best to clinical syndromes like spasms and/or clonus, and thus reflect functionality based on intact autonomic spinal circuitry (i.e. spinal reflexes).

Regarding the motor function we were able to demonstrate that spinal autonomic functionality is also likely to mediate a large amount of the basic forward locomotion function in rats (see chapter 
4). Motor tests which require more supraspinal control, i.e. ladder climbing, motor evoked potentials, and backwards locomotion (on a rotating rod) showed relatively more impairment after moderate or severe $\mathrm{SCl}$ than the basic locomotion tests like open-field, gait-analyses, and the rotating rod. However, these tests were still not able to significantly detect chronic deficits after mild thoracic spinal cord injury. Only one locomotion test, the backwards locomotion test, was able to show significant impairments, and thus sensitivity for loss of (even some) supraspinal control, in mild $\mathrm{SCl}$ at six weeks post injury (see chapter 4).

These findings are important as it is likely that rodent $\mathrm{SCl}$ models can be made more sensitive to treatment effects on restoration of across-lesion connectivity/functionality when outcome measures which are more supra-spinally mediated (such as escape responses and backwards locomotion ability), are used instead of the conventional sensory an motor outcome tests. For patients restoration of across-lesion connectivity and functionality is likely much more relevant than exploiting spinal autonomic function. We therefore conclude that the use of supra-spinally mediated outcome measures, such as escape responses and backward locomotion ability, can render rodent $\mathrm{SCl}$ models more sensitive to detect repair of across-lesion connectivity/functionality.

As the absence of chronic severe locomotor/sensorimotor deficits in rats can be largely ascribed to recovery mediated by the spinal autonomic circuitry, it is argued that this network should 'simply' be trained to regain adequate functionality in human $\mathrm{SCl}$ patients. In this perspective, wheelchair immobilization could have an inhibiting effect on the retraining of spinal autonomic circuitry. This was recently found to be the case in $\mathrm{SCl}$ rats, in which lower body wheelchair immobilization (in rats) led to a vast reduction in recovery of hindpaw function. This indicates that $\mathrm{SCl}$-rats normally highly train their spinal circuitry by moving around in their cages (Caudle 2011). Indeed, it is thought that sensory information associated with phasic limb movements, loading, and paw or foot contact retrains spinal circuitry below the injury (de Leon 2001). Without doubt, some of such spinally mediated sensorimotor functionality is also present in humans (Dietz 2000). However, improvements in walking ability for $\mathrm{SCl}$ patients through step training are variable at best and far less than expected based on animal studies (Dobkin 2007). This might partly be due to the fact that the extensive self-training, as is observed in $\mathrm{SCl}$ rats, is difficult to translate to humans. Nonetheless, the biomechanical properties of bipedalism in humans as compared to quadrupedalism in rats is also much more likely to require a far more complex control, necessitating supraspinal involvement for the multisensory integration and planning to execute motor commands and maintain posture and balance for walking, standing, and sitting (Dobkin 2007). Therefore, the lack of sensitivity for supraspinally mediated functionality of most rodent $\mathrm{SCl}$ outcome tests makes them not ideal to test 
therapeutic efficacy for human $\mathrm{SCl}$ treatments. In view of the fact that the backwards locomotion test did show significant locomotion deficits even in mild SCl animals (see chapter 4) and up to six weeks, we like to state that backwards locomotion is a promising behavioral test for across lesion connectivity and re-connectivity. Which specific spinal motor and/or sensory tracts are most responsible for rat backwards locomotion, remains to be investigated. Interestingly, the corticospinal tract is typically regarded of little importance to basic hindlimb locomotion in rats and other quadrupeds (Drew 2002, Muir 1999), while it has a more predominant role in human voluntary motor functions (including gait) (Barthelemy 2011). This raises the question what the role of the corticospinal tract is for backwards locomotion. If the corticospinal tract is significantly involved in backwards locomotion, then this may result in testing backwards locomotion on a treadmill as a better outcome measure than forward locomotion, in terms of clinical relevance. In view of this, the persistent general belief that treadmill tests per se (like rotating rod tests) less optimally represent voluntary locomotion, as compared to open-field or runway tests, must be questioned. Hence, it is recommended that treadmill testing allows more stringent control and acquisition of testing parameters, for example: speed, partial external weight support, and as we show, direction of locomotion. The use of an automated treadmill with video capturing and some limited fixed restraining of the animal could significantly improve this method.

Another aspect important to consider in view of assessing supraspinal response thresholds in $\mathrm{SCl}$ rats and treatment effects for below-level sensation, is the fact that both increased thresholds (hyposensitivity) as well as decreased thresholds (hypersensitivity) are both undesirable outcomes and that baseline thresholds (i.e. normosensitivity) is the aim. In other words: the closer to the original sensory baseline thresholds of an individual animal, the better the treatment. This seems obvious, but widely used analyses to compare treatment groups (such as repeated measures ANOVAs) tend to ignore this aspect. As group means are used for the analyses, any therapeutic difference between groups in, for example, a better recovery of hyposensitivity in one group might be due to a few animals which developed thresholds below their baseline values (and thus became hypersensitive). The latter might suggest that the recovery is worse, which consequently leads to misinterpretations. We here want to state that an index for the outcome of sensory function should include the following two aspects and as a consequence becomes more appropriate. First, baseline sensory thresholds of individual animals must be used to define hypo- and hyper-sensitivity. Secondly, changes of sensory thresholds are relative to baseline values in a logarithmic relation between stimulus intensity and perceptive thresholds, as generally used is a concept generally in 
most psychometric tests (Chaplan 1994, Mills 2012). Logarithmic transformation of thresholds makes that a relative increase, for example, a twofold increase in perceptive thresholds would always equal a similar (i.e. a twofold) decrease, in absolute terms.

Here we suggest implementing the abovementioned concepts in future studies. The outcome then is based on a single, easy to use, outcome variable, the 'Improvement Index', which we will further explain below:

$$
\text { Improvement Index } x_{i}=\operatorname{abs}\left(\log \left(\frac{x_{i, \text { pretreatment }}}{x_{i, \text { baseline }}}\right)\right)-\operatorname{abs}\left(\log \left(\frac{x_{i, \text { result }}}{x_{i, \text { baseline }}}\right)\right)
$$

Here, $x_{i, t}$ is the average AL-ER threshold of animal $i$ at time point $t$. To improve between-study comparability (regardless of the chosen base of the logarithm) and to help interpret the values of the Improvement Indices, we suggest to also report the reversed log transformations of the groups mean Improvement indices, which, with a log base of 10 , is simply calculated by: $10^{\text {Mean Improvement index }}$. Reversed log transformed Improvement Indices can also be plotted on a secondary $y$-axis (for example, see Fig.IX-2B), to give an idea of the effect size or the net improvement ratio).

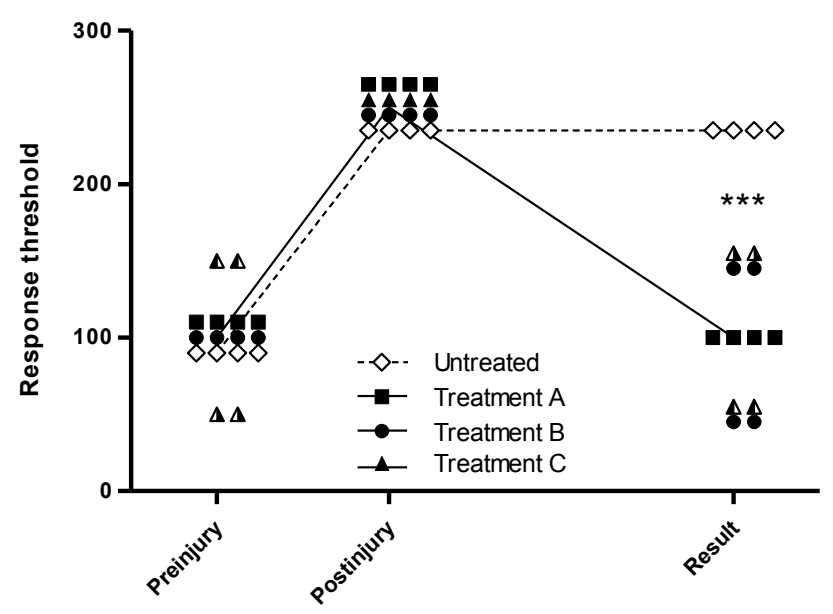

A

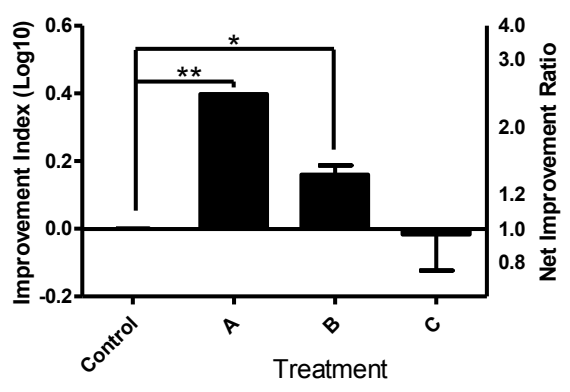

B

Figure IX-2: Example graphs, showing fictive data, to illustrate differences between classical repeated measures analyses (A) and Improvement Indices analyses (B) to explore differences in the treatment effect on sensory thresholds. 
In order to further illustrate the added value of the Improvement Index an example will be given: starting from three experimental $\mathrm{SCl}$ treatment groups, each containing four th. $\mathrm{SCCl}$ rats (Experimental groups A, B, and C in Fig.IX-2A). These experimental groups all have shown post-injury hyposensitivity, when compared to the non-treated control group based on standard statistical analyses (Bonferroni post-hoc: $\mathrm{P}<0.001$, for all). Moreover treatment groups $\mathrm{A}, \mathrm{B}$, and $\mathrm{C}$ do not statistically differ (Two Way Repeated Measures ANOVA: $P=1.00$ ). However, one can easily deduce from the graph (Fig IX-2A) that the outcome of the treatment in group A is better than in groups $B$ and $C$, as only group $A$ has no remaining individuals with hyper- and hypoalgesia. If all individual measurements would merely be logarithmically transformed (which becomes obligatory definitely for data on von Frey mechanical hypersensitivity (Chaplan 1994, Mills 2012)), the differences become more apparent, although still no significance is reached (Two Way Repeated Measures ANOVA: $P=0.56)$. Hence, based on this type of analyses the conclusion is still justified that treatment in group $A, B$ and $C$ are equally effective, thereby completely disregarding the fact that $50 \%$ of the animals in group $B$, and especially of group $C$, become hypersensitive while the other $50 \%$ only partly recovered from their hyposensitivity. With the use of the individually based Improvement Indices and statistical analysis of between-group differences (One-way ANOVA \& Bonferroni post-hoc) mean differences between treatment groups is statistically significant $(P<0.05,95 \%$ confidence intervals: group A vs. B: 0.01-0.46, group B vs. C: 0.001-0.40). Hence, Improvement Indices form a better basis for correct evaluation of treatment results in tests based on sensory thresholds.

The analysis and outcome based on Improvement Indices might have substantial positive impact on the future success of studies into this topic, but it requires quite an elaborate explanation before it can be used and allows appropriate interpretation by the reader. Instead, we have used the percentage of the maximal possible effect (\%MPE), with logarithmically transformed sensory thresholds. Although this method of \%MPE does not use individual baseline and pretreatment values, it does use the group's average baseline and pretreatment value to express the treatment efficacy. Furthermore, \%MPE does not discriminate between hyper-, hypo-, and normo-sensitivity. In the specific case of chapter 6 , the use of \%MPE did not result in different conclusions as compared to those after the use of Improvement Indices. Nevertheless, Improvement Indices can yield very different, and more realistic, results when compared to \%MPE and classic comparisons of the mean, especially in case overall treatment effects result in normo-sensitivity, as in such situations the differences between all conditions (hypo-, hyper-, and normo-sensitivity) become relatively small. 


\section{Spinal Cord Injury research and effective therapies}

The ultimate goal of animal $\mathrm{SCl}$ research is to find effective therapies to test possible effective therapies to improve behavioral outcome related to pain and functional disabilities. It is therefore that we addressed the third research question: "does transplantation of OECs or HSSCs into the lesion site provide functional improvement in an experimental SCI model".

In this thesis we focused our efforts on the use of cell (transplantation) therapies. Two different cell types were used for transplantation; rat olfactory ensheathing cells (OEC) (Chapter 5) and human spinal stem cells (HSSC) (Chapter 6), because both hold great promise in restoration of neurotrauma such as SCl (as detailed in Introduction (Chapter $\mathbf{1}$ ).

\section{Olfactory Ensheating Cells (OEC)}

With respect to the implantation of 3D OEC collagen scaffold complexes into a hemisected spinal cord no functional improvement was noted during a follow-up period of 10 weeks (chapter 5). The acute grafting of a structured 3D collagen scaffold seeded with OEC into a $2 \mathrm{~mm}$-long thoracic spinal cord hemisection rat model, did not result in beneficial nor detrimental effects in terms of gait and withdrawal reflexes. On the other hand, intraparenchymal injection of HSSC into and around a lumbar (L3) spinal cord compression injury 3 days post lesion resulted in some functional improvements in terms of gait, sensory function, and spasticity (stretch hyperreflexia), as measured up to 8 weeks post injury/grafting. Hence, small but significant functional improvements in terms of sensory function, spasticity and gait were observed after the implantation of intraparenchymal injected HSSC cells into the compressed spinal cord.

The absence of a functional therapeutic effect of our 3D OEC scaffold in an $\mathrm{SCl}$ model seems to be related to the inability of OEC to occupy and populate the entire injury site, even in the presence of a state-of-the-art 3D collagen scaffold which provides a bridging opportunity for the OEC. Other studies showing efficacy of OEC therapy in rodent models typically use relative small/gap-less injuries (Li 1997, Li 1998), of which could thus explain why these models did show a functional improvement after OEC therapy. Nevertheless, even our SCl model used is a far less challenging representation of the one found in the human condition (which include random cavitations and uneven lacerations). At the end of our 10 week 3D-OEC scaffold post-grafting experiment, the OEC's could be detected (as they had been obtained from GFP-transgenic rats) and we found the majority of these grafted cells at the border of the graft. Here, the OEC may have exerted a facilitating effect on axonal growth, as neurofilament positive staining was noted within the graft (Fig. IX-3). However it needs to be stressed that neurofilament-immunoreactivity was also observed after implantation of in non-OEC-seeded scaffolds. Clearly, as stated before, the increased neurofilament ingrowth into 
the graft is not accompanied by any behavioral improvement (at 10 weeks post injury). These rather disappointing results may relate to an insufficient in vivo survival or migration capability of OEC upon transplantation. In vitro, we observed OEC throughout the (pre-implanted) scaffolds, including the presence of OEC within the scaffold center (chapter 5). Hence, possible difficulties in optimal nutrient supply to the scaffold-center, necessary for OEC survival in vivo, might underlie the absence of these cells at this location. Unexpectedly, the center of the graft was occupied by neurofilament positive fibers, irrespective of the use of OEC-seeded or non-seeded scaffolds. Therefore, the presence of axonal profiles, throughout and including the center of the graft is not likely related to interactions induced by transplanted OEC.

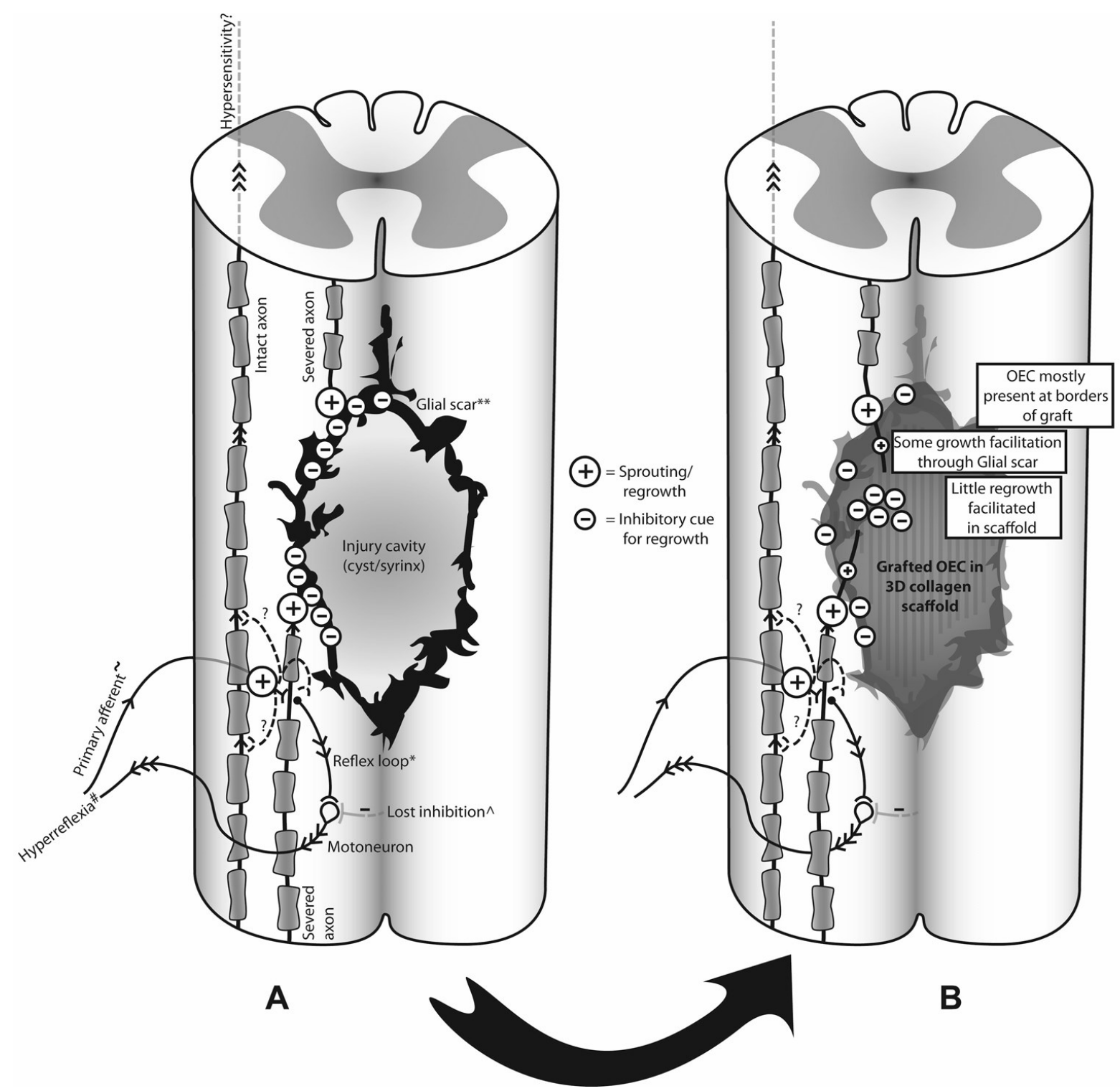

Figure IX-3: Artists drawing of a section of an injured spinal cord, after formation of an injury cavity and glial scar**. Left (A): schematic representation of the clinical problem after SCI (see thesis introduction; chapter I). Shown are severed axons which (endogenously) attempt sprouting/regrowth(+) after injury. However, 
this sprouting is inhibited(-), mainly due to the formation of an astroglial scar**. Sprouting of primary afferents causes part of the spinal (spastic) hyperreflexia ${ }^{\#}$ and possibly also hypersensitivity (/hyperalgesia). Right (B): acute grafting of 3D OEC collagen scaffolds. A few neurofilamentimmunoreactive nerve fibers were found into the graft, but this was not significantly altered by the presence of OEC in the scaffold, at best indicating a minor prevention of the inhibitory effects of the glial scar formation by the scaffold (and OEC?). Also, the center of the scaffold provided little trophic support, as the center of the scaffolds were found to be mostly unpopulated by OEC and, although to a lesser extent, by neurofilaments.

Furthermore, acute post-injury grafting fully exposes the OEC to the secondary injury cascade which follows directly after the primary injury (see chapter 1 ), which then further increases the nutritional and metabolic stress exerted upon them. An early timing of engraftment and nutritionproviding/facilitating capabilities (e.g. collagen density) of the graft are thus issues which could remain to be important factors responsible for our negative results. Timing of the grafting might thus be a critical factor in survival of the transplanted cells and thus in the effect and induction of axonal regrowth.

In contrast, any cell-based therapy used in preliminary studies in human $\mathrm{SCl}$ patients typically report improved therapeutic outcome when grafting is performed early after injury (Sykova 2006, Yoon 2007). The time-frames referred to in these clinical studies are very difficult to relate to the experimental findings. In general engraftment into the lesions of human $\mathrm{SCl}$ patients rarely occurs within the first weeks after injury, and is more often performed many months, up to years, after injury. Moreover, currently published clinical studies on cell-based therapies in SCl typically lack a sufficient number of study subjects and an (appropriate) control group. Interestingly, a first randomized double-blinded controlled (good quality) study of OEC grafted in non-modeled canine $\mathrm{SCl}$ (34 pet dogs) showed some locomotor improvement in OEC treated animals, which was not observed in control (vehicle-injected) animals, even though the treatment was initiated at least more than a year after SCI (Granger 2012, Ito 2006, Krudewig 2006). The OECs used in these canine experiments were harvested from the olfactory mucosa. This is comparable to the OECs used in a fairly successful non-randomized clinical study in SCl patients where gain with only minor pain problems (without any novel persistent pain problems) was shown (Lima 2006). In our experiments we used OEC collected from the olfactory bulb, closely mimicking the OECs used in a clinical study which showed mostly negative results (Huang 2012). Although the present available clinical OEC studies are of low quality in terms of methodological design (e.g. blinding, size, and selection of control groups), the results are suggestive for a possible important difference in clinical impact if the 
OECs implanted are either harvested from the nasal mucosa or the olfactory bulb itself. Another advantage of OEC collected from the olfactory mucosa is that they are much more easily obtained for autologous transplantation, which aids greatly in circumventing host vs. graft immune response problems (as discussed in chapter 8 ).

From this we tentatively conclude that there might be a subgroup of OECs which is able to facilitate functional locomotor recovery. These transplanted OECs might induce neuronal plasticity including axonal regrowth as has been shown in well controlled experimental studies (Li 1997, Novikova 2011). Although (OEC-filled) scaffolds might eventually facilitate across lesion functional (re-) connectivity in a perfectly specific fashion, it does not necessarily provide an answer to problems secondary to the spinal hyperexcitability found after $\mathrm{SCl}$. These problems are believed to be the basis of many clinical problems, such as pain, spasticity, spasms, and (autonomic) hyperreflexia. In fact, an increased neuronal plasticity might induce worsening of secondary problems after $\mathrm{SCl}$ (see Introduction Chapter 1). Even if perfect long tract bridging and reconnectivity can one day be facilitated, it remains an open, and yet mostly theoretical question, whether the problem of hyperexcitable propriospinal circuitry would then also be treated or that it allowed easy facilitation of increased pain and hyper-reflex states. In this context it is important to realize that the onset of problems related to spinal hyperexcitability are unlikely to be solely a direct consequence of a loss of spinal-supraspinal connections. Most secondary problems such as spasticity and pain are likely to be attributed to spinal plasticity, as they develop over time, up to months or years after the primary insult (Hiersemenzel 2000, Siddall 2003). The largest window of opportunity for OEC-therapy thus lies in the early phase after $\mathrm{SCl}$, so it might prevent the onset of secondary complications.

\section{Human Spinal Stem Cells (HSSC)}

Intraparenchymal injection of HSSC into and around a lumbar (L3) spinal cord compression injury 3 days post lesion resulted in significant improvements both on reconnectivity and regeneration of injured fibers as well as on hyperexcitability of segmental networks and development of spasticity and central pain. This is in contrast to the possible effects of OEC, which seem more limited to the improvement of reconnectivity (see above). It has been shown that HSCC engraftment into an animal model of spasticity ameliorates the spinal hyperexcitability and (due to that) locomotion recovery (Cizkova 2007). Furthermore, HSSC have also shown to be able to regenerate/relay long tracts across spinal lesions (Bonner 2011).

Our HSSC transplantation into a traumatic SCl rodent model also resulted in amelioration of spasticity (i.e. stretch evoked muscle hyperreflexia) in $\mathrm{SCl}$ animals, which was possibly due to an increased inhibitory input on the hyperexcitable spinal (reflex) circuitry (see Fig. IX-4B). The 
observed coinciding improvement in locomotion, which consisted of a slight -but significantimprovement in hindlimb paw positioning, might either been due to a secondary effect of the improvement of the spastic-syndrome or to an improvement in supraspinally mediated locomotor control. The latter would suggest some across lesion reconnectivity; a feature which is also supported by our observations as reported in Chapter 6: 1) the observation of an improvement in the $\mathrm{SCl}$-induced below-level hyposensitivity and 2) the wide-spread neuronal differentiation, cavityfilling, and host-graft synaptogenesis of transplanted HSSC (see Fig. IX-4). These observations which are indicative for improved across lesion connectivity, however, remain merely circumstantial evidence, as we have found a) only slight but non-significant improvements in cortical evoked hindlimb MMEPs, b) no difference in (myelinated) axon survival in the spinal white matter. Furthermore it should be noted that the sensory improvements could also indicate the onset of a state of hypersensitivity (hyperalgesia?) rather than an improvement of hyposensitivity. Nevertheless, we observed an amelioration of the spinal hyperexcitability, without onset of belowlevel hypersensitivity in terms of absolute thresholds. Although we did not find evidence of therapyinduced increases in axonal density at the lesion site, it needs mentioning that improved rerouting through existing axons could also still be a mechanism for the recovery of behavior. It has been shown that spontaneous plasticity can occur in the injured rat spinal cord which can facilitate functional recovery across the lesion site through mechanisms of rerouting via still existing axonal pathways (Ballermann 2006, Courtine 2008).

In view of future clinical application of our HSSCs the observations on reconnectivity and regeneration of injured fibers as well as on hyperexcitability of segmental networks and development of spasticity and central pain are important but at least equally important, no tumor formation (see chapters $\mathbf{7}$ and $\mathbf{8}$ ) was observed, with at the same time a pronounced neuronal differentiation of the transplanted HSSCs (neuronal differentiation is regarded a rare quality among different neural stem cells) as well as astroglial and oligodendroglial differentiation with cavity-filling (and syrinx-preventing) potential.

In conclusion: spasticity, hyposensitivity, and paw placement are targeted by our HSSC treatment, without worsening of other outcome in the L3 SCI model. Specifically, HSSC-therapy did not show any signs or symptoms indicative for problems of hyperalgesia. We were not able to demonstrate any direct histological evidence of improved survival of axons or the formations of new neuronal relays across the injury site (although others did show that neuronal precursors do have this capacity (Bonner 2011, Lu 2012)). Nonetheless, similar to the evidenced amelioration in the L3-SCl-induced plastic changes by HSSC engraftment, HSSC could also have facilitated rerouting of lost across-lesion 
connectivity through surviving axons by improving spontaneous plastic changes at both infra- and supra-lesional spinal segments.

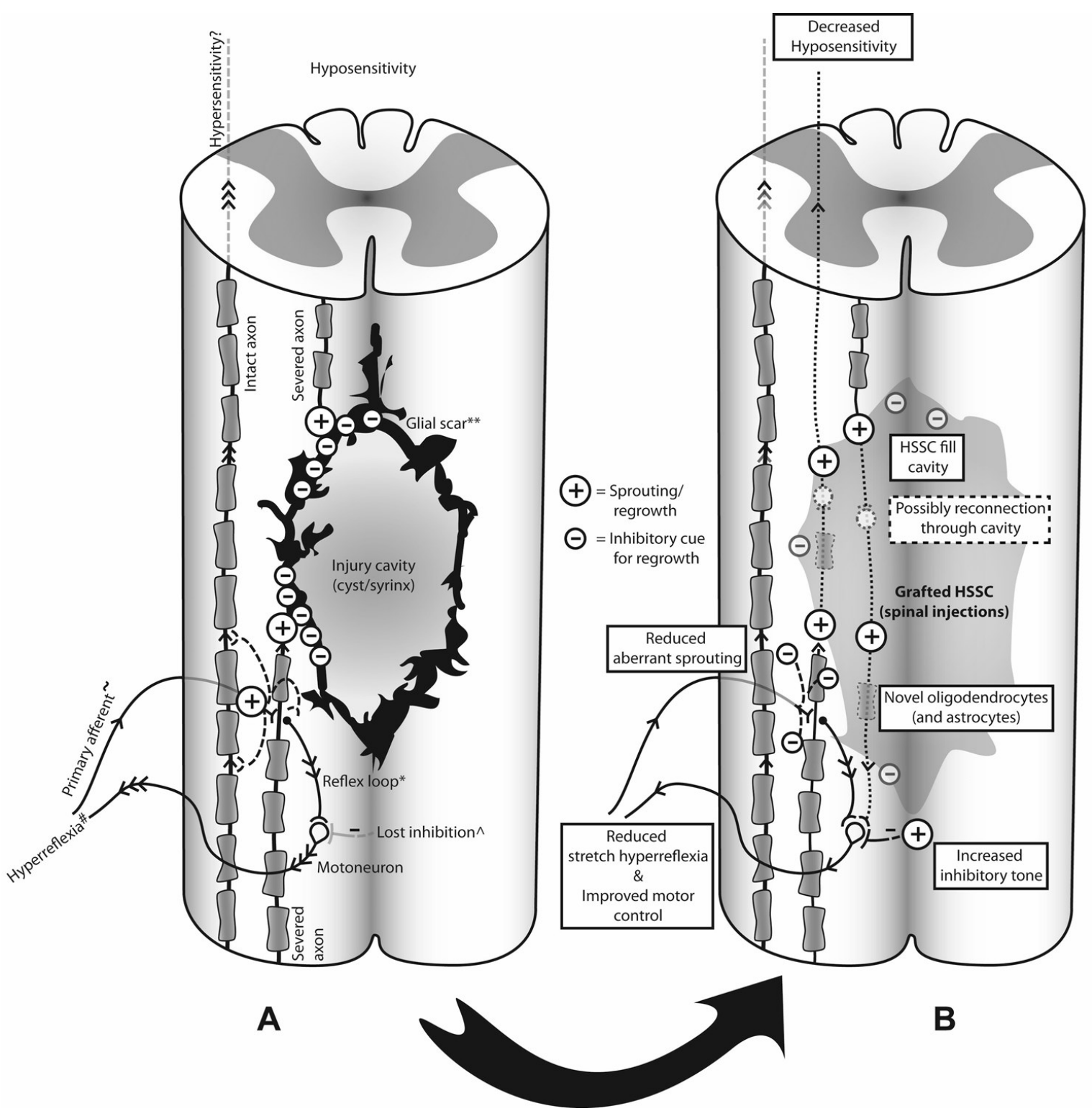

Figure IX-4: Abstract drawing of a section of an injured spinal cord, after formation of an injury cavity and glial scar**. Left (A): schematic of the clinical problem after SCI (see thesis introduction; chapter I). Shown are severed axons, causing motor deficits and hyposensitivity, which are (endogenously) attempting sprouting/regrowth(+) after injury. However, the latter is inhibited(-), mainly due to the glial scar**. Also

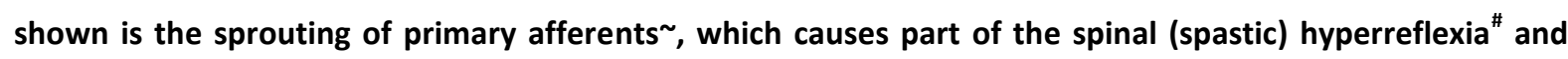
possibly also hypersensitivity (/hyperalgesia). Right (B): the treatment effect of our HSSC treatment, initiated at 3 days post-injury. HSSC 1) filled up most of the cavity, 2) decreased hypoesthesia, and aberrant primary afferent sprouting, 3) led to some improvement stretch hyperreflexia and gait, and 4) were shown to differentiate in I) neuron with synaptic (incl. GABAergic) connectivity on host neurons, II) astrocytes, and III) oligodendrocytes, at $\mathbf{2}$ months post grafting/injury. 


\section{Possible future research goals}

Although our OEC transplantation approach in a rat SCI model did not show any added benefit, the scaffold itself did seem to facilitate some neurofilament outgrowth. It is therefore imaginable that bare scaffolds (or perhaps even seeded with OEC from the nasal mucosa; see IX.3.1) eventually prove useful. Furthermore, adapting the shape (and method of delivery) of scaffolds for use in human injuries, which consist of cavities and non-smooth lacerations, should be prioritized.

The opportunities of effective therapies and $\mathrm{SCl}$ research seems to lie mainly in improving/preventing depression (chapter 2) and in the amelioration of local plasticity, both by improving aberrant sprouting or activity and (possibly) facilitating signal relay or rerouting (see above). Regarding the latter, future pharmacological studies can provide better evidence as for which pathways are most involved in which therapeutic effect, and help selecting and optimizing cell-based therapies. For instance, such a study can answer the question whether it is indeed the GABA-ergic neuronal activity which improves the spasticity, and to what extent. It is also in this perspective of local effectiveness that is was less surprising that we noted benefits in the L3 SCI model (chapter 6), but not in the thoracic SCl model (as in chapter 7), for the HSSC treatment, as the thoracic $\mathrm{SCl}$ model requires the therapeutic effects to be carried out much further from the site of injury, site of cell-grafting, and site of outcome measurement (i.e. the hindpaws). Obviously, when ameliorating local plasticity is the main treatment effect, gaining effects on larger distances is more difficult, especially when the outcome measures (such as locomotor and sensory measures) are merely gross measures and are lacking the required sensitivity to measure small effects. We thus believe and emphasize that with the use of appropriate models cell-based therapies can -and should- be further optimized in such a way that they will prove to be both a real and a predictable benefit for specific problems in human $\mathrm{SCl}$ patients. Notwithstanding, appropriate outcome measures should also be used, for which spasticity measurements, supraspinally mediated sensory and motor tests, and ideally even measures of spontaneous pain, are desirable, but still heavily underused. 
年 


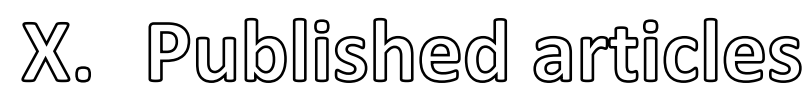

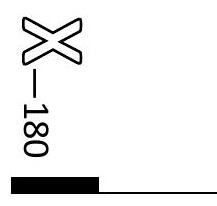




\section{List of published articles (December 2013)}

1. [Chapter 6] van Gorp S, Leerink M, Kakinohana O, Platoshyn O, Santucci C, Galik J, Joosten EA, Hruska-Plochan M, Goldberg D, Marsala S, et al: Amelioration of motor/sensory dysfunction and spasticity in a rat model of acute lumbar spinal cord injury by human neural stem cell transplantation. Stem cell research \& therapy 2013, 4:57.

2. [Chapter 8] Sevc J, Goldberg D, van Gorp S, Leerink M, Juhas S, Juhasova J, Marsala S, Hruska-Plochan M, Hefferan MP, Motlik J, et al: Effective long-term immunosuppression in rats by subcutaneously implanted sustained-release tacrolimus pellet: Effect on spinally grafted human neural precursor survival. Exp Neurol 2013, 248C:85-99.

3. [Chapter 5] Deumens R, van Gorp S, Bozkurt A, Beckmann C, Fuhrmann T, Montzka K, Tolba R, Kobayashi E, Heschel I, Weis J, Brook GA: Motor outcome and allodynia are largely unaffected by novel olfactory ensheathing cell grafts to repair low-thoracic lesion gaps in the adult rat spinal cord. Behav Brain Res 2013, 237:185-189.

4. Kakinohana O, Juhasova J, Juhas S, Motlik J, Platoshyn O, Galik J, Hefferan M, Yuan SH, Vidal JG, Carson CT, Galik J, van Gorp S, et al: Survival and differentiation of human embryonic stem cell-derived neural precursors grafted spinally in spinal ischemia-injured rats or in naive immunosuppressed minipigs: a qualitative and quantitative study. Cell transplantation 2012, 21:2603-2619.

5. Israel MA, Yuan SH, Bardy C, Reyna SM, Mu Y, Herrera C, Hefferan MP, van Gorp S, Nazor KL, Boscolo FS, et al: Probing sporadic and familial Alzheimer's disease using induced pluripotent stem cells. Nature 2012, 482:216-220.

6. Jaken RJ, van Gorp S, Joosten EA, Losen M, Martinez-Martinez P, De Baets M, Marcus MA, Deumens R: Neuropathy-induced spinal GAP-43 expression is not a main player in the onset of mechanical pain hypersensitivity. J Neurotrauma 2011, 28:2463-2473.

\section{Submitted:}

[Chapter 2] van Gorp S, Kessels AG, Joosten EA, van Kleef M, Patijn J: Pain prevalences and their 
[Chapter 3] van Gorp S, Deumens R, Leerink M, Nguyen S, Joosten EA, Marsala M: Translation of the rat thoracic contusion model; part 1 - supraspinally versus spinally-mediated pain-like responses and spasticity. Spinal Cord

[Chapter 4] van Gorp S, Leerink M, Nguyen S, Platoshyn O, Marsala M, Joosten EA: Translation of the rat thoracic contusion model; part $\mathbf{2}$ - forward versus backwards locomotion testing. Spinal Cord 
$\prod_{\substack{\infty \\ \infty}}^{m}$ 


\section{xn. Ref Rerences}

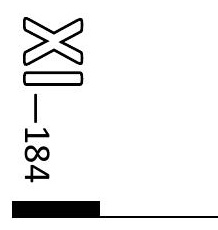


Abematsu M, Tsujimura K, Yamano M, Saito M, Kohno K, Kohyama J, Namihira M, Komiya S, Nakashima K: Neurons derived from transplanted neural stem cells restore disrupted neuronal circuitry in a mouse model of spinal cord injury. The Journal of clinical investigation 2010, 120:3255-3266.

Akhtar AZ, Pippin JJ, Sandusky CB: Animal models in spinal cord injury: a review. Reviews in the neurosciences 2008, 19:47-60.

Alexander CL, Fitzgerald UF, Barnett SC: Identification of growth factors that promote long-term proliferation of olfactory ensheathing cells and modulate their antigenic phenotype. Glia 2002, 37:349-364.

Alexander JK, Popovich PG: Neuroinflammation in spinal cord injury: therapeutic targets for neuroprotection and regeneration. Progress in brain research 2009, 175:125-137.

Alexanian AR, Svendsen CN, Crowe MJ, Kurpad SN: Transplantation of human glial-restricted neural precursors into injured spinal cord promotes functional and sensory recovery without causing allodynia. Cytotherapy 2011, 13:61-68.

Amariglio N, Hirshberg A, Scheithauer BW, Cohen Y, Loewenthal R, Trakhtenbrot L, Paz N, KorenMichowitz M, Waldman D, Leider-Trejo L, Toren A, Constantini S, Rechavi G: Donor-derived brain tumor following neural stem cell transplantation in an ataxia telangiectasia patient. PLoS medicine 2009, 6:e1000029.

Anke AG, Stenehjem AE, Stanghelle JK: Pain and life quality within 2 years of spinal cord injury. Paraplegia 1995, 33:555-559.

Ankeny DP, Popovich PG: Mechanisms and implications of adaptive immune responses after traumatic spinal cord injury. Neuroscience 2009, 158:1112-1121.

Anson CA, Shepherd C: Incidence of secondary complications in spinal cord injury. Int J Rehabil Res 1996, 19:55-66.

Apostolova I, Irintchev A, Schachner $M$ : Tenascin-R restricts posttraumatic remodeling of motoneuron innervation and functional recovery after spinal cord injury in adult mice. The Journal of neuroscience : the official journal of the Society for Neuroscience 2006, 26:78497859.

Baastrup C, Maersk-Moller CC, Nyengaard JR, Jensen TS, Finnerup NB: Spinal-, brainstem- and cerebrally mediated responses at- and below-level of a spinal cord contusion in rats: Evaluation of pain-like behavior. Pain.

Baastrup C, Jensen TS, Finnerup NB: Pregabalin attenuates place escape/avoidance behavior in a rat model of spinal cord injury. Brain research 2010a.

Baastrup C, Maersk-Moller CC, Nyengaard JR, Jensen TS, Finnerup NB: Spinal-, brainstem- and cerebrally mediated responses at- and below-level of a spinal cord contusion in rats: evaluation of pain-like behavior. Pain 2010b, 151:670-679.

Bair MJ, Robinson RL, Katon W, Kroenke K: Depression and pain comorbidity: a literature review. Archives of internal medicine 2003, 163:2433-2445.

Ballermann M, Fouad K: Spontaneous locomotor recovery in spinal cord injured rats is accompanied by anatomical plasticity of reticulospinal fibers. The European journal of neuroscience 2006, 23:1988-1996.

Barker RA, Ratcliffe E, McLaughlin M, Richards A, Dunnett SB: A role for complement in the rejection of porcine ventral mesencephalic xenografts in a rat model of Parkinson's disease. The Journal of neuroscience : the official journal of the Society for Neuroscience 2000, 20:34153424.

Barraclough KA, Isbel NM, Johnson DW, Campbell SB, Staatz CE: Once- versus twice-daily tacrolimus: are the formulations truly equivalent? Drugs 2011, 71:1561-1577.

Barrett H, McClelland JM, Rutkowski SB, Siddall PJ: Pain characteristics in patients admitted to hospital with complications after spinal cord injury. Archives of physical medicine and rehabilitation 2003, 84:789-795. 
Barriere G, Leblond H, Provencher J, Rossignol S: Prominent role of the spinal central pattern generator in the recovery of locomotion after partial spinal cord injuries. The Journal of neuroscience : the official journal of the Society for Neuroscience 2008, 28:3976-3987.

Barthelemy D, Grey MJ, Nielsen JB, Bouyer L: Involvement of the corticospinal tract in the control of human gait. Progress in brain research 2011, 192:181-197.

Basso DM, Beattie MS, Bresnahan JC: A sensitive and reliable locomotor rating scale for open field testing in rats. J Neurotrauma 1995, 12:1-21.

Basso DM, Beattie MS, Bresnahan JC: Graded histological and locomotor outcomes after spinal cord contusion using the NYU weight-drop device versus transection. Exp Neurol 1996, 139:244256.

Battistuzzo CR, Callister RJ, Callister R, Galea MP: A systematic review of exercise training to promote locomotor recovery in animal models of spinal cord injury. I Neurotrauma 2012, 29:1600-1613.

Bennett $A D$, Chastain KM, Hulsebosch $C E$ : Alleviation of mechanical and thermal allodynia by CGRP(8-37) in a rodent model of chronic central pain. Pain 2000a, 86:163-175.

Bennett AD, Everhart AW, Hulsebosch CE: Intrathecal administration of an NMDA or a non-NMDA receptor antagonist reduces mechanical but not thermal allodynia in a rodent model of chronic central pain after spinal cord injury. Brain research 2000b, 859:72-82.

Bennett DJ, Gorassini M, Fouad K, Sanelli L, Han Y, Cheng J: Spasticity in rats with sacral spinal cord injury. J Neurotrauma 1999, 16:69-84.

Bennett GJ: What is spontaneous pain and who has it? The journal of pain : official journal of the American Pain Society 2012, 13:921-929.

Berger JV, Knaepen L, Janssen SP, Jaken RJ, Marcus MA, Joosten EA, Deumens R: Cellular and molecular insights into neuropathy-induced pain hypersensitivity for mechanism-based treatment approaches. Brain Res Rev 2011, 67:282-310.

Beric A: Altered sensation and pain in spinal cord injury. In Achievements in Restorative Neurology. Volume 3. Edited by Dimitrijevic MR, Wall PD, Lindblom U. London: Karger; 1990: 27-36

Bernhard M, Gries A, Kremer P, Bottiger BW: Spinal cord injury (SCI)--prehospital management. Resuscitation 2005, 66:127-139.

Biyani A, el Masry WS: Post-traumatic syringomyelia: a review of the literature. Paraplegia 1994, 32:723-731.

Bloemen-Vrencken JH, Post MW, Hendriks JM, De Reus EC, De Witte LP: Health problems of persons with spinal cord injury living in the Netherlands. Disabil Rehabil 2005, 27:1381-1389.

Boido $\mathrm{M}$, Garbossa D, Vercelli A: Early graft of neural precursors in spinal cord compression reduces glial cyst and improves function. J Neurosurg Spine 2011, 15:97-106.

Bonner JF, Connors TM, Silverman WF, Kowalski DP, Lemay MA, Fischer I: Grafted neural progenitors integrate and restore synaptic connectivity across the injured spinal cord. The Journal of neuroscience : the official journal of the Society for Neuroscience 2011, 31:46754686.

Botterell EH, Callaghan JC, Jousse AT: Pain in Paraplegia: Clinical Management and Surgical Treatment. Proc R Soc Med 1954, 47:281-288.

Boulis NM, Federici T, Glass JD, Lunn JS, Sakowski SA, Feldman EL: Translational stem cell therapy for amyotrophic lateral sclerosis. Nature reviews Neurology 2011, 8:172-176.

Bozkurt A, Brook GA, Moellers S, Lassner F, Sellhaus B, Weis J, Woeltje M, Tank J, Beckmann C, Fuchs $P$, Damink LO, Schugner F, Heschel I, Pallua N: In vitro assessment of axonal growth using dorsal root ganglia explants in a novel three-dimensional collagen matrix. Tissue Eng 2007, 13:2971-2979.

Bozkurt A, Deumens R, Beckmann C, Olde Damink L, Schugner F, Heschel I, Sellhaus B, Weis J, Jahnen-Dechent W, Brook GA, Pallua N: In vitro cell alignment obtained with a Schwann cell enriched microstructured nerve guide with longitudinal guidance channels. Biomaterials 2009, 30:169-179. 
Bracken MB, Shepard MJ, Hellenbrand KG, Collins WF, Leo LS, Freeman DF, Wagner FC, Flamm ES, Eisenberg HM, Goodman JH, et al.: Methylprednisolone and neurological function 1 year after spinal cord injury. Results of the National Acute Spinal Cord Injury Study. Journal of neurosurgery 1985, 63:704-713.

Bracken MB, Holford TR: Effects of timing of methylprednisolone or naloxone administration on recovery of segmental and long-tract neurological function in NASCIS 2. Journal of neurosurgery 1993, 79:500-507.

Bracken MB, Shepard MJ, Holford TR, Leo-Summers L, Aldrich EF, Fazl M, Fehlings MG, Herr DL, Hitchon PW, Marshall LF, Nockels RP, Pascale V, Perot PL, Jr., Piepmeier J, Sonntag VK, Wagner F, Wilberger JE, Winn HR, Young W: Methylprednisolone or tirilazad mesylate administration after acute spinal cord injury: 1-year follow up. Results of the third National Acute Spinal Cord Injury randomized controlled trial. Journal of neurosurgery 1998, 89:699-706.

Bracken MB: Steroids for acute spinal cord injury. Cochrane database of systematic reviews (Online) 2012, 1:CD001046.

Bramlett HM, Dietrich WD: Progressive damage after brain and spinal cord injury: pathomechanisms and treatment strategies. Progress in brain research 2007, 161:125-141.

Bretzner F, Plemel JR, Liu J, Richter M, Roskams AJ, Tetzlaff W: Combination of olfactory ensheathing cells with local versus systemic cAMP treatment after a cervical rubrospinal tract injury. Journal of neuroscience research 2010, 88:2833-2846.

Brevig T, Holgersson J, Widner $\mathrm{H}$ : Xenotransplantation for CNS repair: immunological barriers and strategies to overcome them. Trends Neurosci 2000, 23:337-344.

Brodbelt AR, Stoodley MA: Post-traumatic syringomyelia: a review. Journal of clinical neuroscience : official journal of the Neurosurgical Society of Australasia 2003, 10:401-408.

Brooks ME, Brouner R, Ohry A: Long term follow up of spinal cord injury caused by penetrating missiles. Paraplegia 1992, 30:131-134.

Brown A, Weaver LC: The dark side of neuroplasticity. Exp Neurol 2012, 235:133-141.

Bruce JH, Norenberg MD, Kraydieh S, Puckett W, Marcillo A, Dietrich D: Schwannosis: role of gliosis and proteoglycan in human spinal cord injury. J Neurotrauma 2000, 17:781-788.

Bryce TN, Dijkers MP, Ragnarsson KT, Stein AB, Chen B: Reliability of the Bryce/Ragnarsson spinal cord injury pain taxonomy. The journal of spinal cord medicine 2006, 29:118-132.

Bunge RP, Puckett WR, Becerra JL, Marcillo A, Quencer RM: Observations on the pathology of human spinal cord injury. A review and classification of 22 new cases with details from a case of chronic cord compression with extensive focal demyelination. Advances in neurology 1993, 59:75-89.

Burke DC: Pain in paraplegia. Paraplegia 1973, 10:297-313.

Burns AS, O'Connell C: The challenge of spinal cord injury care in the developing world. The journal of spinal cord medicine 2012, 35:3-8.

Cairns DM, Adkins RH, Scott MD: Pain and depression in acute traumatic spinal cord injury: origins of chronic problematic pain? Archives of physical medicine and rehabilitation 1996, 77:329335.

Cardenas DD, Turner JA, Warms CA, Marshall HM: Classification of chronic pain associated with spinal cord injuries. Archives of physical medicine and rehabilitation 2002, 83:1708-1714.

Cardenas DD, Bryce TN, Shem K, Richards JS, Elhefni H: Gender and minority differences in the pain experience of people with spinal cord injury. Archives of physical medicine and rehabilitation 2004, 85:1774-1781.

Carpenter MK, Cui X, Hu ZY, Jackson J, Sherman S, Seiger A, Wahlberg LU: In vitro expansion of a multipotent population of human neural progenitor cells. Exp Neurol 1999, 158:265-278.

Caudle KL, Brown EH, Shum-Siu A, Burke DA, Magnuson TS, Voor MJ, Magnuson DS: Hindlimb immobilization in a wheelchair alters functional recovery following contusive spinal cord injury in the adult rat. Neurorehabilitation and neural repair 2011, 25:729-739. 
Celik EC, Erhan B, Lakse E: The clinical characteristics of neuropathic pain in patients with spinal cord injury. Spinal cord 2012, 50:585-589.

Chang BH, Waternaux C, Lipsitz S: Meta-analysis of binary data: which within study variance estimate to use? Stat Med 2001, 20:1947-1956.

Chaplan SR, Bach FW, Pogrel JW, Chung JM, Yaksh TL: Quantitative assessment of tactile allodynia in the rat paw. J Neurosci Methods 1994, 53:55-63.

Christensen MD, Everhart AW, Pickelman JT, Hulsebosch CE: Mechanical and thermal allodynia in chronic central pain following spinal cord injury. Pain 1996, 68:97-107.

Christensen MD, Hulsebosch CE: Spinal cord injury and anti-NGF treatment results in changes in CGRP density and distribution in the dorsal horn in the rat. Exp Neurol 1997, 147:463-475.

Cizkova D, Kakinohana O, Kucharova K, Marsala S, Johe K, Hazel T, Hefferan MP, Marsala M: Functional recovery in rats with ischemic paraplegia after spinal grafting of human spinal stem cells. Neuroscience 2007, 147:546-560.

Clarke CE, Modarres-Sadeghi H, Twomey JA, Burt AA: Prognostic value of cortical magnetic stimulation in spinal cord injury. Paraplegia 1994, 32:554-560.

Courtine G, Song B, Roy RR, Zhong H, Herrmann JE, Ao Y, Qi J, Edgerton VR, Sofroniew MV: Recovery of supraspinal control of stepping via indirect propriospinal relay connections after spinal cord injury. Nature medicine 2008, 14:69-74.

Craig AR, Hancock KM, Dickson HG: Spinal cord injury: a search for determinants of depression two years after the event. Br J Clin Psychol 1994, 33 ( Pt 2):221-230.

Cruz-Almeida Y, Martinez-Arizala A, Widerstrom-Noga EG: Chronicity of pain associated with spinal cord injury: A longitudinal analysis. J Rehabil Res Dev 2005, 42:585-594.

Cruz-Almeida Y, Felix ER, Martinez-Arizala A, Widerstrom-Noga EG: Pain symptom profiles in persons with spinal cord injury. Pain Med 2009, 10:1246-1259.

Cummings BJ, Uchida N, Tamaki SJ, Salazar DL, Hooshmand M, Summers R, Gage FH, Anderson AJ: Human neural stem cells differentiate and promote locomotor recovery in spinal cordinjured mice. Proceedings of the National Academy of Sciences of the United States of America 2005, 102:14069-14074.

Cummings BJ, Uchida N, Tamaki SJ, Anderson AJ: Human neural stem cell differentiation following transplantation into spinal cord injured mice: association with recovery of locomotor function. Neurol Res 2006, 28:474-481.

David S, Aguayo AJ: Axonal elongation into peripheral nervous system "bridges" after central nervous system injury in adult rats. Science 1981, 214:931-933.

David S, Kroner A: Repertoire of microglial and macrophage responses after spinal cord injury. Nature reviews Neuroscience 2011, 12:388-399.

Davies JE, Huang C, Proschel C, Noble M, Mayer-Proschel M, Davies SJ: Astrocytes derived from glial-restricted precursors promote spinal cord repair. J Biol 2006, 5:7.

Davies JE, Proschel C, Zhang N, Noble M, Mayer-Proschel M, Davies SJ: Transplanted astrocytes derived from BMP- or CNTF-treated glial-restricted precursors have opposite effects on recovery and allodynia after spinal cord injury. $J$ Biol 2008, 7:24.

Davis L, Martin J: Studies upon Spinal Cord Injuries II. The Nature and Treatment of Pain. Journal of neurosurgery 1947, 4:483-491.

de Leon RD, Roy RR, Edgerton VR: Is the recovery of stepping following spinal cord injury mediated by modifying existing neural pathways or by generating new pathways? A perspective. Physical therapy 2001, 81:1904-1911.

Deacon T, Schumacher J, Dinsmore J, Thomas C, Palmer P, Kott S, Edge A, Penney D, Kassissieh S, Dempsey $P$, Isacson $O$ : Histological evidence of fetal pig neural cell survival after transplantation into a patient with Parkinson's disease. Nature medicine 1997, 3:350-353.

Demirel G, Yllmaz H, Gencosmanoglu B, Kesiktas N: Pain following spinal cord injury. Spinal cord $1998,36: 25-28$. 
Department of Agriculture's Code of Federal Regulations 9: Animal Welfare. In Book Animal Welfare (Editor ed.^eds.). City; 2009.

Detloff MR, Fisher LC, McGaughy V, Longbrake EE, Popovich PG, Basso DM: Remote activation of microglia and pro-inflammatory cytokines predict the onset and severity of below-level neuropathic pain after spinal cord injury in rats. Exp Neurol 2008, 212:337-347.

Deumens R, Koopmans GC, Den Bakker CG, Maquet V, Blacher S, Honig WM, Jerome R, Pirard JP, Steinbusch HW, Joosten EA: Alignment of glial cells stimulates directional neurite growth of CNS neurons in vitro. Neuroscience 2004, 125:591-604.

Deumens R, Koopmans GC, Joosten EA: Regeneration of descending axon tracts after spinal cord injury. Progress in neurobiology 2005, 77:57-89.

Deumens R, Koopmans GC, Honig WM, Hamers FP, Maquet V, Jerome R, Steinbusch HW, Joosten EA: Olfactory ensheathing cells, olfactory nerve fibroblasts and biomatrices to promote longdistance axon regrowth and functional recovery in the dorsally hemisected adult rat spinal cord. Exp Neurol 2006a, 200:89-103.

Deumens R, Koopmans GC, Honig WM, Maquet V, Jerome R, Steinbusch HW, Joosten EA: Limitations in transplantation of astroglia-biomatrix bridges to stimulate corticospinal axon regrowth across large spinal lesion gaps. Neuroscience letters 2006b, 400:208-212.

Deumens R, Koopmans GC, Lemmens M, Mollers S, Honig WM, Steinbusch HW, Brook G, Joosten EA: Neurite outgrowth promoting effects of enriched and mixed OEC/ONF cultures. Neuroscience letters 2006c, 397:20-24.

Deumens R, Joosten EA, Waxman SG, Hains BC: Locomotor dysfunction and pain: the scylla and charybdis of fiber sprouting after spinal cord injury. Molecular neurobiology 2008, 37:5263.

Di Lazzaro V, Oliviero A, Profice P, Ferrara L, Saturno E, Pilato F, Tonali P: The diagnostic value of motor evoked potentials. Clinical neurophysiology : official journal of the International Federation of Clinical Neurophysiology 1999, 110:1297-1307.

Di Lazzaro V, Pilato F, Oliviero A, Saturno E, Dileone M, Tonali PA: Role of motor evoked potentials in diagnosis of cauda equina and lumbosacral cord lesions. Neurology 2004, 63:2266-2271.

Dickersin K: Systematic reviews in epidemiology: why are we so far behind? Int J Epidemiol 2002, 31:6-12.

Dietz V, Duysens J: Significance of load receptor input during locomotion: a review. Gait \& posture 2000, 11:102-110.

Dijkers $M$, Bryce $T$, Zanca J: Prevalence of chronic pain after traumatic spinal cord injury: a systematic review. J Rehabil Res Dev 2009, 46:13-29.

Dobkin BH: Confounders in rehabilitation trials of task-oriented training: lessons from the designs of the EXCITE and SCILT multicenter trials. Neurorehabilitation and neural repair 2007, 21:313.

Donnelly C, Eng JJ: Pain following spinal cord injury: the impact on community reintegration. Spinal cord 2005, 43:278-282.

Drew T, Jiang W, Widajewicz W: Contributions of the motor cortex to the control of the hindlimbs during locomotion in the cat. Brain Res Brain Res Rev 2002, 40:178-191.

Dwan K, Altman DG, Arnaiz JA, Bloom J, Chan AW, Cronin E, Decullier E, Easterbrook PJ, Von Elm E, Gamble C, Ghersi D, loannidis JP, Simes J, Williamson PR: Systematic review of the empirical evidence of study publication bias and outcome reporting bias. PLOS ONE 2008, 3:e3081.

Eidelberg E, Walden JG, Nguyen LH: Locomotor control in macaque monkeys. Brain : a journal of neurology 1981, 104:647-663.

Ek CJ, Habgood MD, Callaway JK, Dennis R, Dziegielewska KM, Johansson PA, Potter A, Wheaton B, Saunders NR: Spatio-temporal progression of grey and white matter damage following contusion injury in rat spinal cord. PLoS One 2010, 5:e12021. 
Emerson JD, Burdick E, Hoaglin DC, Mosteller F, Chalmers TC: An empirical study of the possible relation of treatment differences to quality scores in controlled randomized clinical trials. Controlled clinical trials 1990, 11:339-352.

Englund U, Bjorklund A, Wictorin K, Lindvall O, Kokaia M: Grafted neural stem cells develop into functional pyramidal neurons and integrate into host cortical circuitry. Proceedings of the National Academy of Sciences of the United States of America 2002, 99:17089-17094.

Erceg S, Ronaghi M, Oria M, Rosello MG, Arago MA, Lopez MG, Radojevic I, Moreno-Manzano V, Rodriguez-Jimenez FJ, Bhattacharya SS, Cordoba J, Stojkovic M: Transplanted oligodendrocytes and motoneuron progenitors generated from human embryonic stem cells promote locomotor recovery after spinal cord transection. Stem Cells 2010, 28:15411549.

Fagerlund M, Estrada CP, Jaff N, Svensson M, Brundin L: Neural Stem/Progenitor Cells Transplanted to the Hypoglossal Nucleus Integrates with the Host CNS in Adult Rats and Promotes Motor Neuron Survival. Cell transplantation 2011.

Falci S, Holtz A, Akesson E, Azizi M, Ertzgaard P, Hultling C, Kjaeldgaard A, Levi R, Ringden O, Westgren $M$, Lammertse $D$, Seiger A: Obliteration of a posttraumatic spinal cord cyst with solid human embryonic spinal cord grafts: first clinical attempt. J Neurotrauma 1997, 14:875-884.

Faulkner JR, Herrmann JE, Woo MJ, Tansey KE, Doan NB, Sofroniew MV: Reactive astrocytes protect tissue and preserve function after spinal cord injury. The Journal of neuroscience : the official journal of the Society for Neuroscience 2004, 24:2143-2155.

Fawcett JW, Asher RA: The glial scar and central nervous system repair. Brain research bulletin 1999, 49:377-391.

Fenollosa P, Pallares J, Cervera J, Pelegrin F, Inigo V, Giner M, Forner V: Chronic pain in the spinal cord injured: statistical approach and pharmacological treatment. Paraplegia 1993, 31:722729.

Ferrari D, Binda E, De Filippis L, Vescovi AL: Isolation of neural stem cells from neural tissues using the neurosphere technique. Current protocols in stem cell biology 2010, Chapter 2:Unit2D 6.

Festing MFW: Characteristics of nude rats. In Book Characteristics of nude rats (Editor ed.^eds.). pp. 41-49. City: Gustav Fisher; 1982:41-49.

Finn WF: FK506 nephrotoxicity. Ren Fail 1999, 21:319-329.

Finnerup NB, Johannesen IL, Sindrup SH, Bach FW, Jensen TS: Pain and dysesthesia in patients with spinal cord injury: A postal survey. Spinal cord 2001, 39:256-262.

Finnerup NB, Gyldensted C, Nielsen E, Kristensen AD, Bach FW, Jensen TS: MRI in chronic spinal cord injury patients with and without central pain. Neurology 2003a, 61:1569-1575.

Finnerup NB, Johannesen IL, Bach FW, Jensen TS: Sensory function above lesion level in spinal cord injury patients with and without pain. Somatosens Mot Res 2003b, 20:71-76.

Finnerup NB, Johannesen IL, Fuglsang-Frederiksen A, Bach FW, Jensen TS: Sensory function in spinal cord injury patients with and without central pain. Brain : a journal of neurology 2003c, 126:57-70.

Finnerup NB, Sorensen L, Biering-Sorensen F, Johannesen IL, Jensen TS: Segmental hypersensitivity and spinothalamic function in spinal cord injury pain. Exp Neurol 2007, 207:139-149.

Finnerup NB, Baastrup C: Spinal cord injury pain: mechanisms and management. Current pain and headache reports 2012a, 16:207-216.

Finnerup NB, Lund K, Norrbrink C, Trok K, Johannesen IL, Sørensen JC, Jensen TS, Werhagen L: A prospective study of the predictors of pain and sensory changes in the first year following spinal cord injury. Poster session presented at: World Congress on Pain 14th Biannual Conference of the International Association for the Study on Pain 2012b.

Fleming JC, Norenberg MD, Ramsay DA, Dekaban GA, Marcillo AE, Saenz AD, Pasquale-Styles $M$, Dietrich WD, Weaver LC: The cellular inflammatory response in human spinal cords after injury. Brain : a journal of neurology 2006, 129:3249-3269. 
Food and Drug Administration: Guidance for Industry - ICH S6 Preclinical Safety Evaluation of Biotechnology-Derived Pharmaceuticals. In Book Guidance for Industry - ICH S6 Preclinical Safety Evaluation of Biotechnology-Derived Pharmaceuticals (Editor ed.^eds.). City; 1997.

Food Drug Administration, Center for Biologics Evaluation and Research: Points to Consider in the Characterization of Cell Lines Used to Produce Biologicals. In Book Points to Consider in the Characterization of Cell Lines Used to Produce Biologicals (Editor ed.^eds.). City; 1993.

Fortun J, Puzis R, Pearse DD, Gage FH, Bunge MB: Muscle injection of AAV-NT3 promotes anatomical reorganization of CST axons and improves behavioral outcome following $\mathrm{SCl}$. $J$ Neurotrauma 2009, 26:941-953.

Fournier AE, GrandPre T, Strittmatter SM: Identification of a receptor mediating Nogo-66 inhibition of axonal regeneration. Nature 2001, 409:341-346.

Friedenreich CM, Brant RF, Riboli E: Influence of methodologic factors in a pooled analysis of 13 case-control studies of colorectal cancer and dietary fiber. Epidemiology 1994, 5:66-79.

Frisbie JH, Aguilera EJ: Chronic pain after spinal cord injury: an expedient diagnostic approach. Paraplegia 1990, 28:460-465.

Fuhrmann T, Hillen LM, Montzka K, Woltje M, Brook GA: Cell-cell interactions of human neural progenitor-derived astrocytes within a microstructured 3D-scaffold. Biomaterials 2010, 31:7705-7715.

Garcia-Larrea L, Convers P, Magnin M, Andre-Obadia N, Peyron R, Laurent B, Mauguiere F: Laserevoked potential abnormalities in central pain patients: the influence of spontaneous and provoked pain. Brain : a journal of neurology 2002, 125:2766-2781.

Gayman MD, Brown RL, Cui M: Depressive symptoms and bodily pain: The role of physical disability and social stress. Stress and health : journal of the International Society for the Investigation of Stress 2011, 27:52-53.

Gerhart KA, Johnson RL, Whiteneck GG: Health and psychosocial issues of individuals with incomplete and resolving spinal cord injuries. Paraplegia 1992, 30:282-287.

Gijsen VM, de Wildt SN, Tibboel D, Koren G: Tacrolimus-induced nephrotoxicity and genetic variability: A systematic review. Clin Pharmacol Ther 2010, 87:S21-S21.

Glass JD, Boulis NM, Johe K, Rutkove SB, Federici T, Polak M, Kelly C, Feldman EL: Lumbar intraspinal injection of neural stem cells in patients with amyotrophic lateral sclerosis: results of a phase I trial in 12 patients. Stem Cells 2012, 30:1144-1151.

Gold BG, Katoh K, Stormdickerson T: The immunosuppressant FK506 increases the rate of axonal regeneration in rat sciatic nerve. J Neurosci 1995, 15:7509-7516.

Granger N, Blamires H, Franklin RJ, Jeffery ND: Autologous olfactory mucosal cell transplants in clinical spinal cord injury: a randomized double-blinded trial in a canine translational model. Brain : a journal of neurology 2012, 135:3227-3237.

Greenland S: Invited commentary: a critical look at some popular meta-analytic methods. Am J Epidemiol 1994, 140:290-296.

Grillner S: Locomotion in the spinal cat. In Control of posture and locomotion Advances in behavioral biology. Volume 7. Edited by Stein RB, Pearson KG, Smith RS, Redford JB. New York: Plenum Press; 1973: 515-535

Gris $P$, Tighe A, Levin D, Sharma R, Brown A: Transcriptional regulation of scar gene expression in primary astrocytes. Glia 2007, 55:1145-1155.

Gruner JA: A monitored contusion model of spinal cord injury in the rat. J Neurotrauma 1992, 9:123-126; discussion 126-128.

Guo X, Johe K, Molnar P, Davis H, Hickman J: Characterization of a human fetal spinal cord stem cell line, NSI-566RSC, and its induction to functional motoneurons. Journal of tissue engineering and regenerative medicine 2010, 4:181-193.

Gupta N, Solomon J, Raja K: Pain after paraplegia: a survey in India. Spinal cord 2010, 48:342-346. 
Gwak YS, Nam TS, Paik KS, Hulsebosch CE, Leem JW: Attenuation of mechanical hyperalgesia following spinal cord injury by administration of antibodies to nerve growth factor in the rat. Neuroscience letters 2003, 336:117-120.

Gwak YS, Hulsebosch CE: Neuronal hyperexcitability: a substrate for central neuropathic pain after spinal cord injury. Current pain and headache reports 2011a, 15:215-222.

Gwak YS, Hulsebosch CE: GABA and central neuropathic pain following spinal cord injury. Neuropharmacology 2011b, 60:799-808.

Hagg T, Oudega M: Degenerative and spontaneous regenerative processes after spinal cord injury. J Neurotrauma 2006, 23:264-280.

Hains BC, Saab CY, Waxman SG: Changes in electrophysiological properties and sodium channel Nav1.3 expression in thalamic neurons after spinal cord injury. Brain : a journal of neurology 2005, 128:2359-2371.

Hains BC, Waxman SG: Activated microglia contribute to the maintenance of chronic pain after spinal cord injury. The Journal of neuroscience : the official journal of the Society for Neuroscience 2006, 26:4308-4317.

Haisma JA, Van der Woude LH, Stam HJ, Bergen MP, Sluis TA, Post MW, Bussmann JB: Complications following spinal cord injury: occurrence and risk factors in a longitudinal study during and after inpatient rehabilitation. Journal of rehabilitation medicine : official journal of the UEMS European Board of Physical and Rehabilitation Medicine 2007, 39:393-398.

Hall BJ, Lally JE, Vukmanic EV, Armstrong JE, Fell JD, Gupta DS, Hubscher CH: Spinal cord injuries containing asymmetrical damage in the ventrolateral funiculus is associated with a higher incidence of at-level allodynia. The journal of pain : official journal of the American Pain Society 2010, 11:864-875.

Hall ED, Oostveen JA, Gurney ME: Relationship of microglial and astrocytic activation to disease onset and progression in a transgenic model of familial ALS. Glia 1998, 23:249-256.

Halloran PF: Molecular mechanisms of new immunosuppressants. Clin Transplant 1996, 10:118123.

Hamers FP, Lankhorst AJ, van Laar TJ, Veldhuis WB, Gispen WH: Automated quantitative gait analysis during overground locomotion in the rat: its application to spinal cord contusion and transection injuries. J Neurotrauma 2001, 18:187-201.

Hamers FP, Koopmans GC, Joosten EA: CatWalk-assisted gait analysis in the assessment of spinal cord injury. J Neurotrauma 2006, 23:537-548.

Hamm RJ, Pike BR, O'Dell DM, Lyeth BG, Jenkins LW: The rotarod test: an evaluation of its effectiveness in assessing motor deficits following traumatic brain injury. $J$ Neurotrauma 1994, 11:187-196.

Hao JX, Xu XJ, Aldskogius H, Seiger A, Wiesenfeld-Hallin Z: Allodynia-like effects in rat after ischaemic spinal cord injury photochemically induced by laser irradiation. Pain 1991, 45:175-185.

Hari AR, Wydenkeller S, Dokladal P, Halder P: Enhanced recovery of human spinothalamic function is associated with central neuropathic pain after SCl. Exp Neurol 2009, 216:428-430.

Hassanpour K, Hotz-Boendermaker S, Dokladal P, Curt A: Low depressive symptoms in acute spinal cord injury compared to other neurological disorders. Journal of neurology 2012, 259:11421150.

Hasselblad V, Eddy DM, Kotchmar DJ: Synthesis of environmental evidence: nitrogen dioxide epidemiology studies. Journal of the Air \& Waste Management Association 1992, 42:662671.

Hawryluk GW, Mothe A, Wang J, Wang S, Tator C, Fehlings MG: An In Vivo Characterization of Trophic Factor Production Following Neural Precursor Cell or Bone Marrow Stromal Cell Transplantation for Spinal Cord Injury. Stem cells and development 2012. 
Hefferan MP, Johe K, Hazel T, Feldman EL, Lunn JS, Marsala M: Optimization of immunosuppressive therapy for spinal grafting of human spinal stem cells in a rat model of ALS. Cell transplantation 2011, 20:1153-1161.

Hefferan MP, Galik J, Kakinohana O, Sekerkova G, Santucci C, Marsala S, Navarro R, Hruska-Plochan $M$, Johe K, Feldman E, Cleveland DW, Marsala M: Human neural stem cell replacement therapy for amyotrophic lateral sclerosis by spinal transplantation. PLOS One 2012, 7:e42614.

Hendricks WA, Pak ES, Owensby JP, Menta KJ, Glazova M, Moretto J, Hollis S, Brewer KL, Murashov AK: Predifferentiated embryonic stem cells prevent chronic pain behaviors and restore sensory function following spinal cord injury in mice. Mol Med 2006, 12:34-46.

Henningsen P, Lowe B: Depression, pain, and somatoform disorders. Current opinion in psychiatry 2006, 19:19-24.

Heutink M, Post MW, Wollaars MM, van Asbeck FW: Chronic spinal cord injury pain: pharmacological and non-pharmacological treatments and treatment effectiveness. Disabil Rehabil 2011, 33:433-440.

Hiersemenzel LP, Curt A, Dietz V: From spinal shock to spasticity: neuronal adaptations to a spinal cord injury. Neurology 2000, 54:1574-1582.

Higgins JPT, Green S, editors: Cochrane Handbook for Systematic Reviews of Interventions. Version 5.0.1 [updated September 2008]. The Cochrane Collaboration; 2008.

Hitzig SL, Campbell KA, McGillivray CF, Boschen KA, Craven BC: Understanding age effects associated with changes in secondary health conditions in a Canadian spinal cord injury cohort. Spinal cord 2010, 48:330-335.

Hochman S, Gozal EA, Hayes HB, Anderson JT, DeWeerth SP, Chang YH: Enabling techniques for in vitro studies on mammalian spinal locomotor mechanisms. Frontiers in bioscience : a journal and virtual library 2012, 17:2158-2180.

Hoffman JM, Bombardier CH, Graves DE, Kalpakjian CZ, Krause JS: A longitudinal study of depression from 1 to 5 years after spinal cord injury. Archives of physical medicine and rehabilitation 2011, 92:411-418.

Hofstetter CP, Schweinhardt P, Klason T, Olson L, Spenger C: Numb rats walk - a behavioural and fMRI comparison of mild and moderate spinal cord injury. The European journal of neuroscience 2003, 18:3061-3068.

Hofstetter CP, Holmstrom NA, Lilja JA, Schweinhardt P, Hao J, Spenger C, Wiesenfeld-Hallin Z, Kurpad $\mathrm{SN}$, Frisen J, Olson L: Allodynia limits the usefulness of intraspinal neural stem cell grafts; directed differentiation improves outcome. Nat Neurosci 2005, 8:346-353.

Houweling DA, Lankhorst AJ, Gispen WH, Bar PR, Joosten EA: Collagen containing neurotrophin-3 (NT-3) attracts regrowing injured corticospinal axons in the adult rat spinal cord and promotes partial functional recovery. Exp Neurol 1998, 153:49-59.

Huang $\mathrm{H}$, Xi H, Chen L, Zhang F, Liu Y: Long-term outcome of olfactory ensheathing cell therapy for patients with complete chronic spinal cord injury. Cell transplantation 2012, 21 Suppl 1:S23-31.

Huedo-Medina TB, Sanchez-Meca J, Marin-Martinez F, Botella J: Assessing heterogeneity in metaanalysis: Q statistic or 12 index? Psychological methods 2006, 11:193-206.

Hulsebosch CE, Hains BC, Crown ED, Carlton SM: Mechanisms of chronic central neuropathic pain after spinal cord injury. Brain Res Rev 2009, 60:202-213.

Hurlbert RJ: Methylprednisolone for acute spinal cord injury: an inappropriate standard of care. Journal of neurosurgery 2000, 93:1-7.

Ibrahim A, Lee KY, Kanoo LL, Tan CH, Hamid MA, Hamedon NM, Haniff J: Epidemiology of Spinal Cord Injury in Hospital Kuala Lumpur. Spine 2012.

Ito $\mathrm{D}$, Ibanez $\mathrm{C}$, Ogawa $\mathrm{H}$, Franklin RJ, Jeffery ND: Comparison of cell populations derived from canine olfactory bulb and olfactory mucosal cultures. American journal of veterinary research 2006, 67:1050-1056. 
Ivanhoe $\mathrm{CB}$, Reistetter TA: Spasticity: the misunderstood part of the upper motor neuron syndrome. American journal of physical medicine \& rehabilitation / Association of Academic Physiatrists 2004, 83:S3-9.

Jacobs SR, Yeaney NK, Herbison GJ, Ditunno JF, Jr.: Future ambulation prognosis as predicted by somatosensory evoked potentials in motor complete and incomplete quadriplegia. Archives of physical medicine and rehabilitation 1995, 76:635-641.

Jacobs Y, Selden N, Al-Uzri A, Koch T, Vogel H, Huhn S, Uchida N, Dalma-Weiszhausz D, Tsukamoto A, Steiner R: Long-term Survival and Migration of Allogeneic Human Central Nervous System Stem Cells following Intracerebral Transplantation in Neuronal Ceroid Lipofuscinosis. In Book Long-term Survival and Migration of Allogeneic Human Central Nervous System Stem Cells following Intracerebral Transplantation in Neuronal Ceroid Lipofuscinosis (Editor ed.^eds.). City; June 2011.

Jan FK, Wilson PE: A survey of chronic pain in the pediatric spinal cord injury population. The journal of spinal cord medicine 2004, 27 Suppl 1:S50-53.

Jann MW, Slade JH: Antidepressant agents for the treatment of chronic pain and depression. Pharmacotherapy 2007, 27:1571-1587.

Jensen MP, Hoffman AJ, Cardenas DD: Chronic pain in individuals with spinal cord injury: a survey and longitudinal study. Spinal cord 2005, 43:704-712.

Jensen MP, Kuehn CM, Amtmann D, Cardenas DD: Symptom burden in persons with spinal cord injury. Archives of physical medicine and rehabilitation 2007, 88:638-645.

Jin Y, Neuhuber B, Singh A, Bouyer J, Lepore A, Bonner J, Himes T, Campanelli JT, Fischer I: Transplantation of human glial restricted progenitors and derived astrocytes into a contusion model of spinal cord injury. J Neurotrauma 2011, 28:579-594.

Jockenhovel F, Vogel E, Kreutzer M, Reinhardt W, Lederbogen S, Reinwein D: Pharmacokinetics and pharmacodynamics of subcutaneous testosterone implants in hypogonadal men. Clin Endocrinol 1996, 45:61-71.

Johe KK, Hazel TG, Muller T, Dugich-Djordjevic MM, McKay RD: Single factors direct the differentiation of stem cells from the fetal and adult central nervous system. Genes \& development 1996, 10:3129-3140.

Johnson RL, Gerhart KA, McCray J, Menconi JC, Whiteneck GG: Secondary conditions following spinal cord injury in a population-based sample. Spinal cord 1998, 36:45-50.

Jones CF, Cripton PA, Kwon BK: Gross Morphological Changes of the Spinal Cord Immediately After Surgical Decompression in a Large Animal Model of Traumatic Spinal Cord Injury. Spine 2012, 37:E890-E899.

Joosten EA, Veldhuis WB, Hamers FP: Collagen containing neonatal astrocytes stimulates regrowth of injured fibers and promotes modest locomotor recovery after spinal cord injury. Journal of neuroscience research 2004, 77:127-142.

Juni P, Witschi A, Bloch R, Egger M: The hazards of scoring the quality of clinical trials for metaanalysis. JAMA 1999, 282:1054-1060.

Kakinohana O, Cizkova D, Tomori Z, Hedlund E, Marsala S, Isacson O, Marsala M: Region-specific cell grafting into cervical and lumbar spinal cord in rat: a qualitative and quantitative stereological study. Exp Neurol 2004, 190:122-132.

Kakinohana O, Juhasova J, Juhas S, Motlik J, Platoshyn O, Galik J, Hefferan M, Yuan SH, Vidal JG, Carson CT, van Gorp S, Goldberg D, Leerink M, Lazar P, Marsala S, Miyanohara A, Keshavarzi $S$, Ciacci JD, Marsala M: Survival and differentiation of human embryonic stem cell-derived neural precursors grafted spinally in spinal ischemia-injured rats or in naive immunosuppressed minipigs: a qualitative and quantitative study. Cell transplantation 2012, 21:2603-2619.

Kakulas BA, Smith E, Gaekwad U, Kaelan C, Jacobsen PF: The neuropathology of pain and abnormal sensations in human spinal cord injury derived from the clinicopathological data base at the Royal Perth Hospital. In Recent Achievements in Restorative Neurology; Altered 
sensation and pain. Volume 3. Edited by Dimitrijevic MR, Wall PD, Lindblom U. Basel: Karger; 1990: $37-41$

Kalpakjian CZ, Scelza WM, Forchheimer MB, Toussaint LL: Preliminary reliability and validity of a Spinal Cord Injury Secondary Conditions Scale. The journal of spinal cord medicine 2007, 30:131-139.

Kaplan LI, Grynbaum BB, Lloyd KE, Rusk HA: Pain and spasticity in patients with spinal cord dysfunction. Results of a follow-up study. JAMA 1962, 182:918-925.

Keirstead HS, Nistor G, Bernal G, Totoiu M, Cloutier F, Sharp K, Steward O: Human embryonic stem cell-derived oligodendrocyte progenitor cell transplants remyelinate and restore locomotion after spinal cord injury. The Journal of neuroscience : the official journal of the Society for Neuroscience 2005, 25:4694-4705.

Kennedy P, Frankel H, Gardner B, Nuseibeh I: Factors associated with acute and chronic pain following traumatic spinal cord injuries. Spinal cord 1997, 35:814-817.

Kennedy $\mathrm{P}$, Sherlock $\mathrm{O}, \mathrm{McClelland} \mathrm{M}$, Short $\mathrm{D}$, Royle J, Wilson C: A multi-centre study of the community needs of people with spinal cord injuries: the first 18 months. Spinal cord 2010, 48:15-20.

Kim DS, Jung SJ, Nam TS, Jeon YH, Lee DR, Lee JS, Leem JW, Kim DW: Transplantation of GABAergic neurons from ESCs attenuates tactile hypersensitivity following spinal cord injury. Stem Cells 2010, 28:2099-2108.

Kino T, Hatanaka H, Hashimoto M, Nishiyama M, Goto T, Okuhara M, Kohsaka M, Aoki H, Imanaka H: FK-506, a novel immunosuppressant isolated from a streptomyces. 1. fermentation, isolation and physicochemical and biological characteristics. J Antibiot 1987, 40:1249-1255.

Klotz R, Joseph PA, Ravaud JF, Wiart L, Barat M: The Tetrafigap Survey on the long-term outcome of tetraplegic spinal cord injured persons: Part III. Medical complications and associated factors. Spinal cord 2002, 40:457-467.

Knutsdottir S: Spinal cord injuries in Iceland 1973-1989. A follow up study. Paraplegia 1993, 31:6872.

Ko S, Nakajima Y, Kanehiro H, Yoshimura A, Nakano H: The pharmacokinetic benefits of newly developed liposome-incorporated FK506. Transplantation 1994, 58:1142-1144.

Koelsch $A$, Feng $Y$, Fink DJ, Mata M: Transgene-mediated GDNF expression enhances synaptic connectivity and GABA transmission to improve functional outcome after spinal cord contusion. Journal of neurochemistry 2010, 113:143-152.

Koopmans GC, Deumens R, Honig WM, Hamers FP, Steinbusch HW, Joosten EA: The assessment of locomotor function in spinal cord injured rats: the importance of objective analysis of coordination. J Neurotrauma 2005, 22:214-225.

Krahn GL, Suzuki R, Horner-Johnson W: Self-rated health in persons with spinal cord injury: relationship of secondary conditions, function and health status. Qual Life Res 2009, 18:575-584.

Krause JS: Long Term Prediction of Self-reported Problems Following Spinal Cord Injury. Paraplegia 1990, 28:186-202.

Krenz NR, Weaver LC: Nerve growth factor in glia and inflammatory cells of the injured rat spinal cord. Journal of neurochemistry 2000, 74:730-739.

Krudewig C, Deschl U, Wewetzer K: Purification and in vitro characterization of adult canine olfactory ensheathing cells. Cell and tissue research 2006, 326:687-696.

Kuhn WG: The care and rehabilitation of patients with injuries of the spinal cord and cauda equina: Preliminary report on 113 cases. Journal of neurosurgery 1947, 4:40-68.

Lakatos A, Franklin RJ, Barnett SC: Olfactory ensheathing cells and Schwann cells differ in their in vitro interactions with astrocytes. Glia 2000, 32:214-225.

Lama R, Santos F, Algar FJ, Alvarez A, Baamonde C: Lung transplants with tacrolimus and mycophenolate mofetil: A review. Transplant Proc 2003, 35:1968-1973. 
Lamid S, Chia JK, Kohli A, Cid E: Chronic pain in spinal cord injury: comparison between inpatients and outpatients. Archives of physical medicine and rehabilitation 1985, 66:777-778.

Leboeuf-Yde C, Lauritsen JM: The prevalence of low back pain in the literature. A structured review of 26 Nordic studies from 1954 to 1993. Spine 1995, 20:2112-2118.

Lepore AC, Neuhuber B, Connors TM, Han SS, Liu Y, Daniels MP, Rao MS, Fischer I: Long-term fate of neural precursor cells following transplantation into developing and adult CNS. Neuroscience 2006, 142:287-304.

Levi R, Hultling C, Nash MS, Seiger A: The Stockholm spinal cord injury study: 1. Medical problems in a regional SCl population. Paraplegia 1995, 33:308-315.

Li Y, Field PM, Raisman G: Repair of adult rat corticospinal tract by transplants of olfactory ensheathing cells. Science 1997, 277:2000-2002.

Li Y, Field PM, Raisman G: Regeneration of adult rat corticospinal axons induced by transplanted olfactory ensheathing cells. The Journal of neuroscience : the official journal of the Society for Neuroscience 1998, 18:10514-10524.

Li Y, Decherchi P, Raisman G: Transplantation of olfactory ensheathing cells into spinal cord lesions restores breathing and climbing. The Journal of neuroscience : the official journal of the Society for Neuroscience 2003, 23:727-731.

Li Y, Li D, Raisman G: Interaction of olfactory ensheathing cells with astrocytes may be the key to repair of tract injuries in the spinal cord: the 'pathway hypothesis'. I Neurocytol 2005, 34:343-351.

Lima C, Pratas-Vital J, Escada P, Hasse-Ferreira A, Capucho C, Peduzzi JD: Olfactory mucosa autografts in human spinal cord injury: a pilot clinical study. The journal of spinal cord medicine 2006, 29:191-203; discussion 204-196.

Liu J, Wolfe D, Hao S, Huang S, Glorioso JC, Mata M, Fink DJ: Peripherally delivered glutamic acid decarboxylase gene therapy for spinal cord injury pain. Molecular therapy : the journal of the American Society of Gene Therapy 2004, 10:57-66.

Llado J, Haenggeli C, Maragakis NJ, Snyder EY, Rothstein JD: Neural stem cells protect against glutamate-induced excitotoxicity and promote survival of injured motor neurons through the secretion of neurotrophic factors. Mol Cell Neurosci 2004, 27:322-331.

Locker D, Grushka M: Response trends and nonresponse bias in a mail survey of oral and facial pain. Journal of public health dentistry 1988, 48:20-25.

Lopez-Vales R, Fores J, Verdu E, Navarro X: Acute and delayed transplantation of olfactory ensheathing cells promote partial recovery after complete transection of the spinal cord. Neurobiology of disease 2006, 21:57-68.

Lopez-Vales R, Fores J, Navarro X, Verdu E: Chronic transplantation of olfactory ensheathing cells promotes partial recovery after complete spinal cord transection in the rat. Glia 2007, 55:303-311.

Loy DN, Magnuson DS, Zhang YP, Onifer SM, Mills MD, Cao QL, Darnall JB, Fajardo LC, Burke DA, Whittemore SR: Functional redundancy of ventral spinal locomotor pathways. The Journal of neuroscience : the official journal of the Society for Neuroscience 2002, 22:315-323.

Lu P, Jones LL, Snyder EY, Tuszynski MH: Neural stem cells constitutively secrete neurotrophic factors and promote extensive host axonal growth after spinal cord injury. Exp Neurol 2003, 181:115-129.

Lu P, Tuszynski MH: Growth factors and combinatorial therapies for CNS regeneration. Exp Neurol 2008, 209:313-320.

Lu P, Wang Y, Graham L, McHale K, Gao M, Wu D, Brock J, Blesch A, Rosenzweig ES, Havton LA, Zheng $B$, Conner JM, Marsala $M$, Tuszynski MH: Long-distance growth and connectivity of neural stem cells after severe spinal cord injury. Cell 2012, 150:1264-1273.

Lundqvist C, Siosteen A, Blomstrand C, Lind B, Sullivan M: Spinal cord injuries. Clinical, functional, and emotional status. Spine 1991, 16:78-83. 
Lunn JS, Pacut C, Backus C, Hong Y, Johe K, Hefferan M, Marsala M, Feldman EL: The pleotrophic effects of insulin-like growth factor-I on human spinal cord neural progenitor cells. Stem cells and development 2010, 19:1983-1993.

MacGregor MS, Bradley JA: Overview of immunosuppressive therapy in organ transplantation. British journal of hospital medicine 1995, 54:276-284.

Machacova K, Lysack C, Neufeld S: Self-rated health among persons with spinal cord injury: what is the role of physical ability? The journal of spinal cord medicine 2011, 34:265-272.

Macias MY, Syring MB, Pizzi MA, Crowe MJ, Alexanian AR, Kurpad SN: Pain with no gain: allodynia following neural stem cell transplantation in spinal cord injury. Exp Neurol 2006, 201:335348.

Mackay-Sim A, Feron F, Cochrane J, Bassingthwaighte L, Bayliss C, Davies W, Fronek P, Gray C, Kerr G, Licina P, Nowitzke A, Perry C, Silburn PA, Urquhart S, Geraghty T: Autologous olfactory ensheathing cell transplantation in human paraplegia: a 3-year clinical trial. Brain : a journal of neurology 2008, 131:2376-2386.

Magnuson DS, Zhang YP, Cao QL, Han Y, Burke DA, Whittemore SR: Embryonic brain precursors transplanted into kainate lesioned rat spinal cord. Neuroreport 2001, 12:1015-1019.

Magnuson DS, Lovett R, Coffee C, Gray R, Han Y, Zhang YP, Burke DA: Functional consequences of lumbar spinal cord contusion injuries in the adult rat. J Neurotrauma 2005, 22:529-543.

Mannello F, Medda V, Tonti GA: Hypoxia and neural stem cells: from invertebrates to brain cancer stem cells. The International journal of developmental biology 2011, 55:569-581.

Marchand F, Tsantoulas C, Singh D, Grist J, Clark AK, Bradbury EJ, McMahon SB: Effects of Etanercept and Minocycline in a rat model of spinal cord injury. Eur J Pain 2009, 13:673681.

Margot-Duclot A, Tournebise $H$, Ventura M, Fattal C: What are the risk factors of occurence and chronicity of neuropathic pain in spinal cord injury patients? Ann Phys Rehabil Med 2009, 52:111-123.

Mariano AJ: Chronic pain and spinal cord injury. Clin J Pain 1992, 8:87-92.

Marsala M, Hefferan MP, Kakinohana O, Nakamura S, Marsala J, Tomori Z: Measurement of peripheral muscle resistance in rats with chronic ischemia-induced paraplegia or morphine-induced rigidity using a semi-automated computer-controlled muscle resistance meter. J Neurotrauma 2005, 22:1348-1361.

Martino G, Pluchino S: The therapeutic potential of neural stem cells. Nature reviews Neuroscience 2006, 7:395-406.

Matsumoto T, Tamaki T, Kawakami M, Yoshida M, Ando M, Yamada H: Early complications of highdose methylprednisolone sodium succinate treatment in the follow-up of acute cervical spinal cord injury. Spine 2001, 26:426-430.

McAlister VC: Liposomal tacrolimus: Drug migration within blood compartments. Transplant Proc 1998, 30:1000-1001.

McDonald JW, Liu XZ, Qu Y, Liu S, Mickey SK, Turetsky D, Gottlieb DI, Choi DW: Transplanted embryonic stem cells survive, differentiate and promote recovery in injured rat spinal cord. Nature medicine 1999, 5:1410-1412.

McDonald JW, Sadowsky C: Spinal-cord injury. Lancet 2002, 359:417-425.

McKeon F: When worlds collide - Immunosuppressant meet protein phosphatases. Cell 1991, 66:823-826.

McKeon RJ, Hoke A, Silver J: Injury-induced proteoglycans inhibit the potential for lamininmediated axon growth on astrocytic scars. Exp Neurol 1995, 136:32-43.

McKinley W, Sinha A, Ketchum J, Deng X: Comparison of rehabilitation outcomes following vascular-related and traumatic spinal cord injury. The journal of spinal cord medicine 2011, 34:410-415.

McKinley WO, Johns JS, Musgrove JJ: Clinical presentations, medical complications, and functional outcomes of individuals with gunshot wound-induced spinal cord injury. American journal 
of physical medicine \& rehabilitation / Association of Academic Physiatrists 1999, 78:102107.

McKinley WO, Gittler MS, Kirshblum SC, Stiens SA, Groah SL: Spinal cord injury medicine. 2. Medical complications after spinal cord injury: Identification and management. Archives of physical medicine and rehabilitation 2002a, 83:S58-64, S90-58.

McKinley WO, Tewksbury MA, Godbout CJ: Comparison of medical complications following nontraumatic and traumatic spinal cord injury. The journal of spinal cord medicine 2002b, 25:88-93.

Meade MA, Barrett K, Ellenbogen PS, Jackson MS: Work intensity and variations in health and personal characteristics of individuals with spinal cord injury (SCI). J Vocat Rehabil 2006, 25:13-19.

Melzack R, Loeser JD: Phantom body pain in paraplegics: evidence for a central "pattern generating mechanism" for pain. Pain 1978, 4:195-210.

Meyers AR, Bisbee A, Winter M: The "Boston model" of managed care and spinal cord injury: a cross-sectional study of the outcomes of risk-based, prepaid, managed care. Archives of physical medicine and rehabilitation 1999, 80:1450-1456.

Miladinovic K: [Spinal cord injury and chronic pain]. Med Arh 2009, 63:106-107.

Mills C, Leblond D, Joshi S, Zhu C, Hsieh G, Jacobson P, Meyer M, Decker M: Estimating efficacy and drug ED50's using von Frey thresholds: impact of weber's law and log transformation. The journal of pain : official journal of the American Pain Society 2012, 13:519-523.

Mitsui T, Shumsky JS, Lepore AC, Murray M, Fischer I: Transplantation of neuronal and glial restricted precursors into contused spinal cord improves bladder and motor functions, decreases thermal hypersensitivity, and modifies intraspinal circuitry. The Journal of neuroscience : the official journal of the Society for Neuroscience 2005, 25:9624-9636.

Miyamoto Y, Uno T, Yamamoto H, Li XK, Sakamoto K, Hashimoto H, Takenaka H, Kawashima Y, Kawarasaki $\mathrm{H}$ : Pharmacokinetic and immunosuppressive effects of tacrolimus-loaded biodegradable microspheres. Liver Transplant 2004, 10:392-396.

Modirian E, Pirouzi P, Soroush M, Karbalaei-Esmaeili S, Shojaei H, Zamani H: Chronic pain after spinal cord injury: results of a long-term study. Pain Med 2010, 11:1037-1043.

Moher D, Pham B, Jones A, Cook DJ, Jadad AR, Moher M, Tugwell P, Klassen TP: Does quality of reports of randomised trials affect estimates of intervention efficacy reported in metaanalyses? Lancet 1998, 352:609-613.

Mollers S, Heschel I, Damink LH, Schugner F, Deumens R, Muller B, Bozkurt A, Nava JG, Noth J, Brook GA: Cytocompatibility of a novel, longitudinally microstructured collagen scaffold intended for nerve tissue repair. Tissue Eng Part A 2009, 15:461-472.

Mueller BK, Mack $\mathrm{H}$, Teusch N: Rho kinase, a promising drug target for neurological disorders. Nature reviews Drug discovery 2005, 4:387-398.

Muir GD, Whishaw IQ: Complete locomotor recovery following corticospinal tract lesions: measurement of ground reaction forces during overground locomotion in rats. Behav Brain Res 1999, 103:45-53.

Munoz-Quiles C, Santos-Benito FF, Llamusi MB, Ramon-Cueto A: Chronic spinal injury repair by olfactory bulb ensheathing glia and feasibility for autologous therapy. Journal of neuropathology and experimental neurology 2009, 68:1294-1308.

Munro D: Rehabilitation of veterans paralyzed as the result of injury to the spinal cord and cauda equina. Am J Surg 1948, 75:3-18.

Munro D: Two-year end-results in the total rehabilitation of veterans with spinal-cord and caudaequina injuries. $N$ Engl J Med 1950, 242:1-16.

Musienko PE, Zelenin PV, Lyalka VF, Gerasimenko YP, Orlovsky GN, Deliagina TG: Spinal and supraspinal control of the direction of stepping during locomotion. The Journal of neuroscience : the official journal of the Society for Neuroscience 2012, 32:17442-17453. 
National Research Council: Guide for the Care and Use of Laboratory Animals. In Book Guide for the Care and Use of Laboratory Animals (Editor ed.^eds.). City: National Academy Press; 1996.

Navarro R, Juhas S, Keshavarzi S, Juhasova J, Motlik J, Johe K, Marsala S, Scadeng M, Lazar P, Tomori Z, Schulteis G, Beattie M, Ciacci JD, Marsala M: Chronic spinal compression model in minipigs: a systematic behavioral, qualitative, and quantitative neuropathological study. $J$ Neurotrauma 2012, 29:499-513.

Nepomuceno C, Fine PR, Richards JS, Gowens H, Stover SL, Rantanuabol U, Houston R: Pain in patients with spinal cord injury. Archives of physical medicine and rehabilitation 1979, 60:605-609.

Neuralstem Inc. - Emory University: A Phase I, Open-label, First in Human, Feasibility and Safety Study of Human Spinal Cord Derived Neural Stem Cell Transplantation for the Treatment of Amyotrophic Lateral Sclerosis - Identifier: NCT01348451 - cited: 2012 Aug 10. ClinicalTrialsgov [Internet] National Library of Medicine (US) Bethesda (MD) 2000.

New PW, Lim TC, Hill ST, Brown DJ: A survey of pain during rehabilitation after acute spinal cord injury. Spinal cord 1997, 35:658-663.

New PW, Rawicki HB, Bailey MJ: Nontraumatic spinal cord injury: demographic characteristics and complications. Archives of physical medicine and rehabilitation 2002, 83:996-1001.

New PW, McFarlane CL: Retrospective case series of outcomes following spinal cord infarction. Eur J Neurol 2012, 19:1207-1212.

Nicholson Perry K, Nicholas MK, Middleton J: Spinal cord injury-related pain in rehabilitation: a cross-sectional study of relationships with cognitions, mood and physical function. Eur J Pain 2009, 13:511-517.

Noldus LP, Spink AJ, Tegelenbosch RA: EthoVision: a versatile video tracking system for automation of behavioral experiments. Behavior research methods, instruments, \& computers : a journal of the Psychonomic Society, Inc 2001, 33:398-414.

Norrbrink Budh C, Lund I, Ertzgaard P, Holtz A, Hultling C, Levi R, Werhagen L, Lundeberg T: Pain in a Swedish spinal cord injury population. Clin Rehabil 2003, 17:685-690.

Novikova LN, Lobov S, Wiberg M, Novikov LN: Efficacy of olfactory ensheathing cells to support regeneration after spinal cord injury is influenced by method of culture preparation. Exp Neurol 2011, 229:132-142.

Okada S, Nakamura M, Katoh H, Miyao T, Shimazaki T, Ishii K, Yamane J, Yoshimura A, Iwamoto Y, Toyama Y, Okano H: Conditional ablation of Stat3 or Socs3 discloses a dual role for reactive astrocytes after spinal cord injury. Nature medicine 2006, 12:829-834.

Ondarza $A B, Y e Z$, Hulsebosch $C E$ : Direct evidence of primary afferent sprouting in distant segments following spinal cord injury in the rat: colocalization of GAP-43 and CGRP. Exp Neurol 2003, 184:373-380.

Oudega M: Molecular and cellular mechanisms underlying the role of blood vessels in spinal cord injury and repair. Cell and tissue research 2012, 349:269-288.

Packard MJ: Use of slow-release pellets to administer calcitriol to avian embryos - effects on plasma calcium, magnesium and phosphorus. Gen Comp Endocrinol 1992, 85:8-16.

Pagliacci MC, Franceschini M, Di Clemente B, Agosti M, Spizzichino L: A multicentre follow-up of clinical aspects of traumatic spinal cord injury. Spinal cord 2007, 45:404-410.

Pirsch JD, Miller J, Deierhoi MH, Vincenti F, Filo RS: A comparison of tacrolimus (FK506) and cyclosporine for immunosuppression after cadaveric renal transplantation. Transplantation 1997, 63:977-983.

Post MW, De Witte LP, Van Asbeck FW, Van Dijk AJ, Schrijvers AJ: Predictors of health status and life satisfaction in spinal cord injury. Archives of physical medicine and rehabilitation 1998, 79:395-401.

Prysak GM, M. AE, Meyers AR: Prevalence of Secondary Conditions in Veterans with Spinal Cord Injury and Their Interference with Life Activities. Top Spinal Cord Inj Rehabil 2000, 6:34-42. 
Przepiorka D, Devine S, Fay J, Uberti J, Wingard J: Practical considerations in the use of tacrolimus for allogeneic marrow transplantation. Bone Marrow Transplant 1999, 24:1053-1056.

Purves AM, Penny KI, Munro C, Smith BH, Grimshaw J, Wilson B, Smith WC, Chambers WA: Defining chronic pain for epidemiological research : assessing a subjective definition. Pain Clinic 1998, 10:139-147.

Putzke JD, Richards JS, DeVivo MJ: Quality of life after spinal cord injury caused by gunshot. Archives of physical medicine and rehabilitation 2001, 82:949-954.

Putzke JD, Barrett JJ, Richards JS, DeVivo MJ: Age and spinal cord injury: an emphasis on outcomes among the elderly. The journal of spinal cord medicine 2003a, 26:37-44.

Putzke JD, Richards JS, Ness T, Kezar L: Interrater reliability of the International Association for the Study of Pain and Tunks' spinal cord injury pain classification schemes. American journal of physical medicine \& rehabilitation / Association of Academic Physiatrists 2003b, 82:437-440.

Ragnarsson KT: Management of pain in persons with spinal cord injury. The journal of spinal cord medicine 1997, 20:186-199.

Raichle KA, Hanley M, Jensen MP, Cardenas DD: Cognitions, coping, and social environment predict adjustment to pain in spinal cord injury. The journal of pain : official journal of the American Pain Society 2007, 8:718-729.

Raisman G, Li Y: Repair of neural pathways by olfactory ensheathing cells. Nature reviews Neuroscience 2007, 8:312-319.

Raissi GR, Mokhtari A, Mansouri K: Reports from spinal cord injury patients: eight months after the 2003 earthquake in Bam, Iran. American journal of physical medicine \& rehabilitation / Association of Academic Physiatrists 2007, 86:912-917.

Ramon-Cueto A, Avila J: Olfactory ensheathing glia: properties and function. Brain research bulletin 1998, 46:175-187.

Ramon-Cueto A, Cordero MI, Santos-Benito FF, Avila J: Functional recovery of paraplegic rats and motor axon regeneration in their spinal cords by olfactory ensheathing glia. Neuron 2000, 25:425-435.

Ravenscroft A, Ahmed YS, Burnside IG: Chronic pain after SCl. A patient survey. Spinal cord 2000, 38:611-614.

Reis A, Reinhard T, Sundmacher R, Braunstein C, Godehardt E: Effect of mycophenolate mofetil, cyclosporin $\mathrm{A}$, and both in combination in a murine corneal graft rejection model. $\mathrm{Br} \mathrm{J}$ Ophthalmol 1998, 82:700-703.

Richards JS, Stover SL, Jaworski T: Effect of bullet removal on subsequent pain in persons with spinal cord injury secondary to gunshot wound. Journal of neurosurgery 1990, 73:401-404.

Richter MW, Fletcher PA, Liu J, Tetzlaff W, Roskams AJ: Lamina propria and olfactory bulb ensheathing cells exhibit differential integration and migration and promote differential axon sprouting in the lesioned spinal cord. The Journal of neuroscience : the official journal of the Society for Neuroscience 2005, 25:10700-10711.

Rintala DH, Hart KA, Fuhrer MJ: self-reported pain in persons with chronic spinal cord injury [Abstract only]. J Am Paraplegia Soc 1991, 14:83.

Rintala DH, Loubser PG, Castro J, Hart KA, Fuhrer MJ: Chronic pain in a community-based sample of men with spinal cord injury: prevalence, severity, and relationship with impairment, disability, handicap, and subjective well-being. Archives of physical medicine and rehabilitation 1998, 79:604-614.

Rintala DH, Hart KA, Priebe MM: Predicting consistency of pain over a 10-year period in persons with spinal cord injury. J Rehabil Res Dev 2004, 41:75-88.

Rintala DH, Holmes SA, Fiess RN, Courtade D, Loubser PG: Prevalence and characteristics of chronic pain in veterans with spinal cord injury. J Rehabil Res Dev 2005, 42:573-584.

Robertson CE, Brown RD, Jr., Wijdicks EF, Rabinstein AA: Recovery after spinal cord infarcts: longterm outcome in 115 patients. Neurology 2012, 78:114-121.

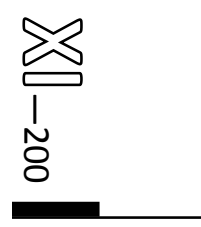


Rose $M$, Robinson JE, Ells $P$, Cole JD: Pain following spinal cord injury: results from a postal survey. Pain 1988, 34:101-102.

Rosenzweig ES, Courtine G, Jindrich DL, Brock JH, Ferguson AR, Strand SC, Nout YS, Roy RR, Miller DM, Beattie MS, Havton LA, Bresnahan JC, Edgerton VR, Tuszynski MH: Extensive spontaneous plasticity of corticospinal projections after primate spinal cord injury. Nature neuroscience 2010, 13:1505-1510.

Rossignol S, Frigon A: Recovery of locomotion after spinal cord injury: some facts and mechanisms. Annual review of neuroscience 2011a, 34:413-440.

Rossignol S, Frigon A, Barriere G, Martinez M, Barthelemy D, Bouyer L, Belanger M, Provencher J, Chau C, Brustein E, Barbeau H, Giroux N, Marcoux J, Langlet C, Alluin O: Chapter 16--spinal plasticity in the recovery of locomotion. Progress in brain research 2011b, 188:229-241.

Rota Nodari L, Ferrari D, Giani F, Bossi M, Rodriguez-Menendez V, Tredici G, Delia D, Vescovi AL, De Filippis $L$ : Long-term survival of human neural stem cells in the ischemic rat brain upon transient immunosuppression. PLoS One 2010, 5:e14035.

Rothstein JD, Dykes-Hoberg M, Pardo CA, Bristol LA, Jin L, Kuncl RW, Kanai Y, Hediger MA, Wang Y, Schielke JP, Welty DF: Knockout of glutamate transporters reveals a major role for astroglial transport in excitotoxicity and clearance of glutamate. Neuron 1996, 16:675-686.

Rupp I, Triemstra M, Boshuizen HC, Jacobi CE, Dinant HJ, van den Bos GA: Selection bias due to nonresponse in a health survey among patients with rheumatoid arthritis. European journal of public health 2002, 12:131-135.

Saab $\mathrm{CY}$, Hains $\mathrm{BC}$ : Remote neuroimmune signaling: a long-range mechanism of nociceptive network plasticity. Trends Neurosci 2009, 32:110-117.

Sahni V, Kessler JA: Stem cell therapies for spinal cord injury. Nature reviews Neurology 2010, 6:363-372.

Saikkonen J, Karppi P, Huusko TM, Dahlberg A, Makinen J, Uutela T: Life situation of spinal cordinjured persons in Central Finland. Spinal cord 2004, 42:459-465.

Saunders LL, Krause JS, Focht KL: A longitudinal study of depression in survivors of spinal cord injury. Spinal cord 2012, 50:72-77.

Saxena K, Patro N, Patro I: FK506 protects neurons following peripheral nerve injury via immunosuppression. Cellular and molecular neurobiology 2007, 27:1049-1057.

Schmidt CO, Raspe H, Pfingsten M, Hasenbring M, Basler HD, Eich W, Kohlmann T: Does attrition bias longitudinal population-based studies on back pain? Eur J Pain 2011, 15:84-91.

Schouenborg J, Holmberg H, Weng HR: Functional organization of the nociceptive withdrawal reflexes. II. Changes of excitability and receptive fields after spinalization in the rat. Exp Brain Res 1992, 90:469-478.

Schulz KF, Chalmers I, Hayes RJ, Altman DG: Empirical evidence of bias. Dimensions of methodological quality associated with estimates of treatment effects in controlled trials. JAMA 1995, 273:408-412.

Schuurman HJ: The nude rat. Human and Experimental Toxicology 1995, 14:122-125.

Semler J, Wellmann K, Wirth F, Stein G, Angelova S, Ashrafi M, Schempf G, Ankerne J, Ozsoy O, Ozsoy $U$, Schonau E, Angelov DN, Irintchev A: Objective measures of motor dysfunction after compression spinal cord injury in adult rats: correlations with locomotor rating scores. $J$ Neurotrauma 2011, 28:1247-1258.

Setoguchi T, Nakashima K, Takizawa T, Yanagisawa M, Ochiai W, Okabe M, Yone K, Komiya S, Taga T: Treatment of spinal cord injury by transplantation of fetal neural precursor cells engineered to express BMP inhibitor. Exp Neurol 2004, 189:33-44.

Sheng $\mathrm{H}$, Wang H, Homi HM, Spasojevic I, Batinic-Haberle I, Pearlstein RD, Warner DS: A nolaminectomy spinal cord compression injury model in mice. J Neurotrauma 2004, 21:595603.

Siddall P, Yezierski RP, Loeser JD: Pain Following Spinal Cord Injury: Clinical Features, Prevalence, and Taxonomy. IASP newsletter 2000, 3:1-8. 
Siddall PJ, Taylor DA, Cousins MJ: Classification of pain following spinal cord injury. Spinal cord 1997, 35:69-75.

Siddall PJ, Taylor DA, McClelland JM, Rutkowski SB, Cousins MJ: Pain report and the relationship of pain to physical factors in the first 6 months following spinal cord injury. Pain 1999, 81:187-197.

Siddall PJ, Yezierski RP, Loeser JD: Taxonomy and epidemiology of spinal cord injury pain. In Spinal Cord Injury Pain: Assessment, Mechanisms, Management Progress in Pain Research and Management. Volume 23. Edited by Burchiel KJ, Yezierski RP. Seattle IASP Press; 2002: 9-24

Siddall PJ, McClelland JM, Rutkowski SB, Cousins MJ: A longitudinal study of the prevalence and characteristics of pain in the first 5 years following spinal cord injury. Pain 2003, 103:249257.

Siekierka JJ, Hung SHY, Poe M, Lin CS, Sigal NH: A cytosolic binding-protein for immunosuppressant FK506 has peptidyl-prolyl isomerase activity but is distinct from cyclophilin. Nature 1989, 341:755-757.

Silver J, Miller JH: Regeneration beyond the glial scar. Nature reviews Neuroscience 2004, 5:146156.

Simard JM, Tsymbalyuk O, Keledjian K, Ivanov A, Ivanova S, Gerzanich V: Comparative effects of glibenclamide and riluzole in a rat model of severe cervical spinal cord injury. Exp Neurol 2012, 233:566-574.

Srinivasan V, Pendergrass JA, Kumar KS, Landauer MR, Seed TM: Radioprotection, pharmacokinetic and behavioural studies in mouse implanted with biodegradable drug (amifostine) pellets. Int J Radiat Biol 2002, 78:535-543.

Staatz CE, Tett SE: Clinical pharmacokinetics and pharmacodynamics of tacrolimus in solid organ transplantation. Clin Pharmacokinet 2004, 43:623-653.

StemCells Inc.: Clinical Trials with 'huCNS-SC'; NLM database search results. ClinicalTrialsgov [Internet] National Library of Medicine (US) Bethesda (MD) 2000 - cited: 2012 Aug 10.

Stensman R: Adjustment to traumatic spinal cord injury. A longitudinal study of self-reported quality of life. Paraplegia 1994, 32:416-422.

Stormer S, Gerner HJ, Gruninger W, Metzmacher K, Follinger S, Wienke C, Aldinger W, Walker N, Zimmermann $\mathrm{M}$, Paeslack V: Chronic pain/dysaesthesiae in spinal cord injury patients: results of a multicentre study. Spinal cord 1997, 35:446-455.

Stroup DF, Berlin JA, Morton SC, Olkin I, Williamson GD, Rennie D, Moher D, Becker BJ, Sipe TA, Thacker SB: Meta-analysis of observational studies in epidemiology: a proposal for reporting. Meta-analysis Of Observational Studies in Epidemiology (MOOSE) group. JAMA 2000, 283:2008-2012.

Studd J, Magos A: Hormone pellet implantation for menopause and premenstrual-syndome. Obstet Gynecol Clin N Am 1987, 14:229-249.

Su VCH, Greanya ED, Ensom MHH: Impact of mycophenolate mofetil dose reduction on allograft outcomes in kidney transplant recipients on tacrolimus-based regimens: a systematic review. Ann Pharmacother 2011, 45:248-257.

Su Z, Yuan Y, Chen J, Cao L, Zhu Y, Gao L, Qiu Y, He C: Reactive astrocytes in glial scar attract olfactory ensheathing cells migration by secreted TNF-alpha in spinal cord lesion of rat. PLoS One 2009, 4:e8141.

Summers JD, Rapoff MA, Varghese G, Porter K, Palmer RE: Psychosocial factors in chronic spinal cord injury pain. Pain 1991, 47:183-189.

Svendsen CN, ter Borg MG, Armstrong RJ, Rosser AE, Chandran S, Ostenfeld T, Caldwell MA: A new method for the rapid and long term growth of human neural precursor cells. J Neurosci Methods 1998, 85:141-152.

Sykova E, Jendelova P, Urdzikova L, Lesny P, Hejcl A: Bone marrow stem cells and polymer hydrogels--two strategies for spinal cord injury repair. Cell Mol Neurobiol 2006, 26:11131129. 
Tan M, New PW: Retrospective study of rehabilitation outcomes following spinal cord injury due to tumour. Spinal cord 2012, 50:127-131.

Tator $\mathrm{CH}$ : Review of treatment trials in human spinal cord injury: issues, difficulties, and recommendations. Neurosurgery 2006, 59:957-982; discussion 982-957.

Teasell RW, Mehta S, Aubut JL, Ashe MC, Sequeira K, Macaluso S, Tu L: A systematic review of the therapeutic interventions for heterotopic ossification after spinal cord injury. Spinal cord 2010, 48:512-521.

Teh LK, Dom SHM, Zakaria ZA, Salleh MZ: A systematic review of the adverse effects of tacrolimus in organ transplant patients. Afr J Pharm Pharmacol 2011, 5:764-771.

Thompson SG: Why sources of heterogeneity in meta-analysis should be investigated. BMJ 1994, 309:1351-1355.

Thomson AW, Bonham CA, Zeevi A: Mode of action of tacrolimus (FK506) - Molecular and cellular mechanisms. Ther Drug Monit 1995, 17:584-591.

Thuret S, Moon LD, Gage FH: Therapeutic interventions after spinal cord injury. Nature reviews Neuroscience 2006, 7:628-643.

Tillakaratne NJ, Guu JJ, de Leon RD, Bigbee AJ, London NJ, Zhong H, Ziegler MD, Joynes RL, Roy RR, Edgerton VR: Functional recovery of stepping in rats after a complete neonatal spinal cord transection is not due to regrowth across the lesion site. Neuroscience 2010, 166:23-33.

Turner JA, Cardenas DD: Chronic pain problems in individuals with spinal cord injuries. Semin Clin Neuropsychiatry 1999, 4:186-194.

Turner JA, Cardenas DD, Warms CA, McClellan CB: Chronic pain associated with spinal cord injuries: a community survey. Archives of physical medicine and rehabilitation 2001, 82:501-509.

Tze WJ, Tai J, Cheung SSC, Ricordi C, Starzl TE: FK 506 - An effective immunosuppressant for islet xenotransplantation. Transplant Proc 1992, 24:2849-2850.

U.S. National Archives and Records Administration: Code of Federal Regulations. Title 21 section 211. Current good mamufacturing practice for finished pharmaceuticals. In Book Code of Federal Regulations. Title 21 section 211. Current good mamufacturing practice for finished pharmaceuticals (Editor ed.^eds.), vol. 4. City; 2012a.

U.S. National Archives and Records Administration: Code of Federal Regulations. Title 21 section 312.23. Investigational New Drug Application. In Book Code of Federal Regulations. Title 21 section 312.23. Investigational New Drug Application (Editor ed.^eds.), vol. 5. City; 2012b.

U.S. National Archives and Records Administration: Code of Federal Regulations. Title 21 section 58. Good laboratory practice for nonclinical laboratory studies. In Book Code of Federal Regulations. Title 21 section 58. Good laboratory practice for nonclinical laboratory studies (Editor ed.^eds.), vol. 1. City; 2012c.

U.S. National Archives and Records Administration: Code of Federal Regulations. Title 21 section 210. Current good manufacturing practice in manufacturing, processing, packing, or holding of drugs; general. In Book Code of Federal Regulations. Title 21 section 210. Current good manufacturing practice in manufacturing, processing, packing, or holding of drugs; general (Editor ed.^eds.), vol. 4. City; 2012d.

Uchida N, Buck DW, He D, Reitsma MJ, Masek M, Phan TV, Tsukamoto AS, Gage FH, Weissman IL: Direct isolation of human central nervous system stem cells. Proceedings of the National Academy of Sciences of the United States of America 2000, 97:14720-14725.

Ullrich PM, Smith BM, Poggensee L, Evans CT, Stroupe KT, Weaver FM, Burns SP: Pain and PostTraumatic Stress Disorder Symptoms During Inpatient Rehabilitation Among Operation Enduring Freedom/Operation Iraqi Freedom Veterans With Spinal Cord Injury. Archives of physical medicine and rehabilitation 2012.

Usvald D, Vodicka P, Hlucilova J, Prochazka R, Motlik J, Kuchorova K, Johe K, Marsala S, Scadeng M, Kakinohana O, Navarro R, Santa M, Hefferan MP, Yaksh TL, Marsala M: Analysis of dosing regimen and reproducibility of intraspinal grafting of human spinal stem cells in immunosuppressed minipigs. Cell transplantation 2010, 19:1103-1122. 
van Gorp S, Leerink M, Kakinohana O, Platoshyn O, Santucci C, Galik J, Joosten EA, Hruska-Plochan M, Goldberg D, Marsala S, Johe K, Ciacci JD, Marsala M: Amelioration of motor/sensory dysfunction and spasticity in a rat model of acute lumbar spinal cord injury by human neural stem cell transplantation. Stem cell research \& therapy 2013, 4:57.

van Loo MA, Post MW, Bloemen JH, van Asbeck FW: Care needs of persons with long-term spinal cord injury living at home in the Netherlands. Spinal cord 2010, 48:423-428.

van Loveren $\mathrm{H}$, Schuurman HJ, Vos JG: Immune deficiency syndrome in rodents: the nude rat. Dordrecht: Martinus Nijhoff; 1988.

Vandertop WP, Notermans NC, Algra A: [Methylprednisolone in traumatic spinal cord injuries: not proven to be beneficial to the patient at the present time]. Nederlands tijdschrift voor geneeskunde 1998, 142:1061-1064.

Vescovi AL, Parati EA, Gritti A, Poulin P, Ferrario M, Wanke E, Frolichsthal-Schoeller P, Cova L, Arcellana-Panlilio $M$, Colombo A, Galli R: Isolation and cloning of multipotential stem cells from the embryonic human CNS and establishment of transplantable human neural stem cell lines by epigenetic stimulation. Exp Neurol 1999, 156:71-83.

Vicari-Christensen M, Repper S, Basile S, Young D: Tacrolimus: review of pharmacokinetics, pharmacodynamics, and pharmacogenetics to facilitate practitioners' understanding and offer strategies for educating patients and promoting adherence. Progress in Transplantation 2009, 19:277-284.

Vierck CJ: Animal models of pain. In Wall and Melzack's Textbook of Pain. Edited by McMahon S, Koltzenburg M. Philadelphia: Elsevier Churchill Livingstone; 2006: 175-186

Vierck CJ, Jr., Lee CL, Willcockson HH, Kitzmiller A, Bullitt E, Light AR: Effects of anterolateral spinal lesions on escape responses of rats to hindpaw stimulation. Somatosensory \& motor research 1995, 12:163-174.

Ville I, Ravaud JF: Subjective well-being and severe motor impairments: the Tetrafigap survey on the long-term outcome of tetraplegic spinal cord injured persons. Soc Sci Med 2001, 52:369-384.

Vogel LC, Krajci KA, Anderson CJ: Adults with pediatric-onset spinal cord injury: part 2: musculoskeletal and neurological complications. The journal of spinal cord medicine 2002, 25:117-123.

Walker BF: The prevalence of low back pain: a systematic review of the literature from 1966 to 1998. J Spinal Disord 2000, 13:205-217.

Walter JS, Sacks J, Othman R, Scianna J, Nemchausky B, Chintam R, Anfinsen L: Characteristics of persons with spinal cord injury as documented by use of an interactive data system. The journal of spinal cord medicine 2000, 23:257-262.

Walter JS, Sacks J, Othman R, Rankin AZ, Nemchausky B, Chintam R, Wheeler JS: A database of selfreported secondary medical problems among VA spinal cord injury patients: its role in clinical care and management. J Rehabil Res Dev 2002, 39:53-61.

Wang D, Bodley R, Sett P, Gardner B, Frankel H: A clinical magnetic resonance imaging study of the traumatised spinal cord more than 20 years following injury. Paraplegia 1996, 34:65-81.

Wang $Q$, Uno T, Miyamoto $Y$, Hara $Y$, Kitazawa $Y$, Lu FZ, Funeshima N, Fujino M, Yamamoto $H$, Takenaka H, Kawashima Y, Li XK: Biodegradable microsphere-loaded tacrolimus enhanced the effect on mice islet allograft and reduced the adverse effect on insulin secretion. American journal of transplantation : official journal of the American Society of Transplantation and the American Society of Transplant Surgeons 2004, 4:721-727.

Wasner G, Lee BB, Engel S, McLachlan E: Residual spinothalamic tract pathways predict development of central pain after spinal cord injury. Brain : a journal of neurology 2008, 131:2387-2400.

Wente MN, Sauer P, Mehrabi A, Weitz J, Buchler MW, Schmidt J, Schemmer P: Review of the clinical experience with a modified release form of tacrolimus [FK506E (MR4)] in transplantation. Clin Transplant 2006, 20:80-84. 
Werhagen L, Budh CN, Hultling C, Molander C: Neuropathic pain after traumatic spinal cord injury-relations to gender, spinal level, completeness, and age at the time of injury. Spinal cord 2004, 42:665-673.

Werhagen L, Hultling C, Molander C: The prevalence of neuropathic pain after non-traumatic spinal cord lesion. Spinal cord 2007, 45:609-615.

Wessels M, Lucas C, Eriks I, de Groot S: Body weight-supported gait training for restoration of walking in people with an incomplete spinal cord injury: a systematic review. Journal of rehabilitation medicine : official journal of the UEMS European Board of Physical and Rehabilitation Medicine 2010, 42:513-519.

White TE, Lane MA, Sandhu MS, O'Steen BE, Fuller DD, Reier PJ: Neuronal progenitor transplantation and respiratory outcomes following upper cervical spinal cord injury in adult rats. Exp Neurol 2010, 225:231-236.

Widerstrom-Noga EG, Felipe-Cuervo E, Broton JG, Duncan RC, Yezierski RP: Perceived difficulty in dealing with consequences of spinal cord injury. Archives of physical medicine and rehabilitation 1999, 80:580-586.

Widerstrom-Noga EG, Finnerup NB, Siddall PJ: Biopsychosocial perspective on a mechanisms-based approach to assessment and treatment of pain following spinal cord injury. $J$ Rehabil Res Dev 2009, 46:1-12.

Wirth ED, 3rd, Reier PJ, Fessler RG, Thompson FJ, Uthman B, Behrman A, Beard J, Vierck CJ, Anderson DK: Feasibility and safety of neural tissue transplantation in patients with syringomyelia. J Neurotrauma 2001, 18:911-929.

Wollaars MM, Post MW, Van Asbeck FW, Brand N: Spinal cord injury pain: the influence of psychologic factors and impact on quality of life. Clin J Pain 2007, 23:383-391.

Woodhall E, West AK, Chuah MI: Cultured olfactory ensheathing cells express nerve growth factor, brain-derived neurotrophic factor, glia cell line-derived neurotrophic factor and their receptors. Brain research Molecular brain research 2001, 88:203-213.

Woolsey RM: Chronic pain following spinal cord injury. J Am Paraplegia Soc 1986, 9:39-41.

Wyndaele $\mathrm{M}$, Wyndaele JJ: Incidence, prevalence and epidemiology of spinal cord injury: what learns a worldwide literature survey? Spinal cord 2006, 44:523-529.

Xu G, Wang L, Chen W, Xue F, Bai X, Liang L, Shen X, Zhang M, Xia D, Liang T: Rapamycin and tacrolimus differentially modulate acute graft-versus-host disease in rats after liver transplantation. Liver transplantation : official publication of the American Association for the Study of Liver Diseases and the International Liver Transplantation Society 2010, 16:357363.

Xu L, Yan J, Chen D, Welsh AM, Hazel T, Johe K, Hatfield G, Koliatsos VE: Human neural stem cell grafts ameliorate motor neuron disease in SOD-1 transgenic rats. Transplantation 2006, 82:865-875.

Yamauchi A, Oishi R, Kataoka Y: Tacrolimus-induced neurotoxicity and nephrotoxicity is ameliorated by administration in the dark phase in rats. Cellular and molecular neurobiology 2004, 24:695-704.

Yan J, Welsh AM, Bora SH, Snyder EY, Koliatsos VE: Differentiation and tropic/trophic effects of exogenous neural precursors in the adult spinal cord. J Comp Neurol 2004, 480:101-114.

Yan J, Xu L, Welsh AM, Chen D, Hazel T, Johe K, Koliatsos VE: Combined immunosuppressive agents or CD4 antibodies prolong survival of human neural stem cell grafts and improve disease outcomes in amyotrophic lateral sclerosis transgenic mice. Stem Cells 2006, 24:1976-1985.

Yan J, Xu L, Welsh AM, Hatfield G, Hazel T, Johe K, Koliatsos VE: Extensive neuronal differentiation of human neural stem cell grafts in adult rat spinal cord. PLoS medicine 2007, 4:e39.

Yap EC, Tow A, Menon EB, Chan KF, Kong KH: Pain during in-patient rehabilitation after traumatic spinal cord injury. Int J Rehabil Res 2003, 26:137-140.

Yezierski RP, Vierck CJ: Reflex and pain behaviors are not equivalent: lessons from spinal cord injury. Pain 2010, 151:569-570. 
Yoon SH, Shim YS, Park YH, Chung JK, Nam JH, Kim MO, Park HC, Park SR, Min BH, Kim EY, Choi BH, Park $\mathrm{H}, \mathrm{Ha}$ Y: Complete spinal cord injury treatment using autologous bone marrow cell transplantation and bone marrow stimulation with granulocyte macrophage-colony stimulating factor: Phase I/II clinical trial. Stem Cells 2007, 25:2066-2073.

Yuan SH, Martin J, Elia J, Flippin J, Paramban RI, Hefferan MP, Vidal JG, Mu Y, Killian RL, Israel MA, Emre N, Marsala S, Marsala M, Gage FH, Goldstein LS, Carson CT: Cell-surface marker signatures for the isolation of neural stem cells, glia and neurons derived from human pluripotent stem cells. PLoS One 2011, 6:e17540.

Zeilig G, Enosh S, Rubin-Asher D, Lehr B, Defrin R: The nature and course of sensory changes following spinal cord injury: predictive properties and implications on the mechanism of central pain. Brain : a journal of neurology 2012, 135:418-430.

Zhao P, Waxman SG, Hains BC: Modulation of thalamic nociceptive processing after spinal cord injury through remote activation of thalamic microglia by cysteine cysteine chemokine ligand 21. The Journal of neuroscience : the official journal of the Society for Neuroscience 2007, 27:8893-8902. 
i

$\stackrel{3}{3}$ 


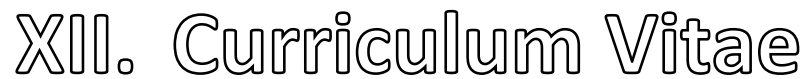

Sebastiaan Franciscus Johannes "Bart" van Gorp was born in Breda, the Netherlands, in 1984 on Friday the $30^{\text {th }}$ of March. From 1996 to 2002 he attended the Sint-Oelbertgymnasium in Oosterhout, Noord-Brabant. In 2002 he started university. First, Biomedical engineering at the Eindhoven University of Technology and, one year later, he transferred to the Maastricht University to study Medicine. During his six years of Medical training, Sebastiaan followed the International Track in Medicine and volunteered in research projects of the Department of Anesthesiology at the Maastricht University Medical Center. He also attended both a research internship at the Montpellier Neuroscience Institute in France and a course on Health Care Systems at Birmingham University in England. From 2009 to 2013 he was a PhD-student at the Department of Anesthesiology at the Maastricht University Medical Center, during which he worked for about two years at the University of California, San-Diego (United States) in the laboratory for neuroregeneration led by Prof. Martin Marsala. Since February 2013 he is working as an Anesthesiology and Reanimation resident at the Université Catholique de Louvain in Brussels, Belgium. 
옥

छ 


\section{Summary}

Current $\mathrm{SCl}$ treatment options are limited. Promising treatments to promote regrowth and regeneration in the injured spinal cord carry the risk of worsening outcomes, such as pain, which is likely due to further stimulating aberrant neural plasticity. However, little is known on the mechanisms of $\mathrm{SCl}$ pain or aberrant neural plasticity. Clues to its etiology might be found by analyzing differences between studies on different $\mathrm{SCl}$ populations, as widely varying pain prevalences have been reported. On the other hand, one can also accept the limited understanding of aberrant neural plasticity etiology and take a perhaps more pragmatic path by screening the currently most promising regenerative treatments, such as cell-based-therapies, in an adequate preclinical in vivo model. However, translation of positive preclinical results into the clinical setting has thus far been disappointing, which calls for a reevaluation of our preclinical tools.

Therefore, this dissertation 1) explores causes for the heterogeneity in $\mathrm{SCl}$ pain prevalences and searches for pain determinants, 2) explores tools (sensory and motor assays, and immunosuppression protocols) for the preclinical and translational evaluation of $\mathrm{SCl}$ treatments, and 3) investigates the relevance and feasibility of promising candidate cell-based therapies (olfactory ensheathing cells, OEC, and human fetal spinal cord-derived neural stem cells, HSSC) for $\mathrm{SCl}$ treatment in preclinical models. The research questions asked are:

1. Is it possible to identify which study and/or population characteristics show a relationship with the differences in reported pain prevalence rates in $\mathrm{SCl}$ patient populations? (chapter 2)

2. Can we differentiate between spinal reflex mediated functionality and supraspinally mediated functionality in rodent SCI models? (chapter 3 and 4)

3. Does transplantation of OEC or HSSC into the lesion site provide functional improvement in an experimental $\mathrm{SCl}$ model? (chapter 5 and 6)

4. Is long-term HSSC engraftment safe for clinical use and achievable with the use of slowrelease immunosuppressive drug formulas? (chapter 7 and 8)

Ad 1) The answer is yes. Eighty-two studies reporting on SCl pain prevalence were examined. Study design related determinants of $\mathrm{SCl}$ pain prevalence reports were: pain definition strictness (mild, moderate, or high), primary study goal (pain study or not), data source (retrospective or not), and, in 
a limited number of cases, response/attrition rates. While correcting for latter items, populated characteristics found to determine pain prevalence rates were both the proportion of patients with a depression and the average time after injury (positive correlations). Between-study heterogeneity may remain even after the identification/correction of abovementioned causes of heterogeneity. Hence, pain after $\mathrm{SCl}$ seems to relate to the duration of the injury and depression, yet, major causes of bias in reported pain prevalence are found to be related to the primary study goal (pain study or not), choice of pain definition, and the use of retrospective data.

Ad 2) The answer is yes. Reduced hindpaw withdrawal thresholds (after rat thoracic SCI), which are often believed to model below-level pain states in human Spinal cord Injury patients do not reflect any decrease in thresholds for pain perception (i.e. hyperalgesia), but instead, coincide with a decrease in below-level perception (i.e. hypoesthesia; which, indeed, is also more the rule than the exception in human $\mathrm{SCl}$ patients, and a limiting factor in recovery of locomotion). Yet, increased hindpaw withdrawal responses might still relate to another clinical problem in $\mathrm{SCl}$ patients; the "spastic"- or "upper motor neuron"-syndrome (which is thus far largely ignored in translational research). Hence, it was tested whether spasticity/muscle stretch hyperreflexia co-occurred with the increased hindpaw responses, but no such spasticity was observed. Therefore we suggest/discuss that hindpaw withdrawal responses are best interpreted as either 1) a different subset of clinical problems related to the spastic syndrome (i.e. spasms and/or clonus), or 2) as mostly a speciesspecific phenomenon. Also, widely-used existing locomotion tests can be questioned for their translational value, as, unlike human $\mathrm{SCl}$ patients, rats 1 ) easily recovers hindpaw walking ability after severe $\mathrm{SCl}, 2$ ) can even do this without sensory perception (see above), 3) have a vastly easier (more stable) bodily position during walking (quadrupedalism vs erect bipedalism), 4) require merely simple 'stepping patterns' for normal/daily-life function, and 5) learn to walk much faster in the postnatal period. This indicates that rat locomotion has a low dependence on spinal integrity or supra-spinal input (i.e. depend on their "Central Pattern Generator" or "Spinal" walking), while humans are much more dependent on supra-spinal control. During preclinical therapy evaluations this difference should be kept in mind and an adequate/translatable testing paradigm which is sensitive for supra-spinal mediated motor function must be used. A novel locomotion test is proposed in this dissertation, i.e. backward walking on a rotating rod, and is shown to be more sensitive to $\mathrm{SCl}$ than existing tests, even in the chronic phase post $\mathrm{SCl}$, and is likely to be more dependent on supra-spinal input.

Ad 3) The answer is 'possibly not' and 'yes', respectively. OEC are a promising graftable cell-based therapy, but only few studies have focused on experimental models with large cavitations (as occurs 
in humans), which will require bridging substrates to transfer and maintain OEC within the lesion site. A state-of-the-art collagen-based multi-channeled three dimensional scaffold is used to deliver olfactory ensheathing cells to $2 \mathrm{~mm}$ long unilateral low-thoracic hemisection cavities in rats. Hyperreflexia of the hindpaws was monitored using the Von Frey hair filament test, while an extensive analysis of motor ability was performed. No substantial improvement or deterioration of motor functions was induced and there was no effect on lesion-induced hyperreflexia. On the basis of these data, it can be concluded that relatively large spinal cord lesions with cavitation may present additional hurdles to the therapeutic effect of OEC. Also, intraspinal grafting of clinical grade HSSC in a rat model of acute lumbar (L3) SCl was tested. Spasticity, lesion volume, various motor and sensory dysfunctions, and neural integration was assessed. Treatment led to a progressive improvement in lower extremity paw placement, amelioration of spasticity and thermal and tactile pain/escape thresholds. Near complete injury-cavity-filling by grafted cells and development of putative GABA-ergic synapses between grafted and host neurons was observed.

Ad 4) The answer is very likely 'yes'. Achievement of effective, safe and long-term immunosuppression represents one of the challenges in experimental allogeneic and xenogeneic cell and organ transplantation. A reliable, long-term immunosuppression protocol in rats was developed by: 1) comparing the pharmacokinetics of four different subcutaneously delivered tacrolimus formulations (tacrolimus is a widely clinically used immunosuppressive agent which inhibits Tlymphocyte signal transduction and IL-2 transcription), and 2) defining the survival and immune response in animals receiving spinal injections of human neural precursors. It is demonstrated that use of TAC pellets can represent an effective, long-lasting immunosuppressive drug delivery system that is safe, simple to implement and is associated with a long-term spinal graft survival in $\mathrm{SCl}$ or SOD+ rats. Furthermore, especially the potential tumorigenic property of many contemporary experimental stem cell therapies requires special preclinical safety assessments, but no standard guidelines or any stem cell safety studies have yet been published. A preclinical assessment of the safety of HSSC therapy for a phase 1 clinical trial application is described. The design of the reported safety study is the result of a dialogue between researchers in this field and the national drug authority (the Food and Drug Administration). The negative results found for tumorigenicity, toxicity, and worsening outcomes, in conjunction with data from HSSC efficacy/therapeutic studies have resulted in the approval of the currently ongoing clinical trial of spinally applied HSSC in human patients. 
$\stackrel{m}{\stackrel{n}{*}}$ 
KUV. AcknoWled:gnemis

The thesis which lies here in front of you, is the result of quite some work, motivation, aspiration, and enthausiasm, but, above all, a great team (in its most broadest meaning). This thesis would definitely not have reached its current state if I could not have relied on the wholehearted support of many others. Therefore, I would like to proclaim this page as the most important page of my thesis, and use this page to express my sincere words of thanks to all who have supported me, in any way, in finalizing this thesis: thank you all!

In this context, I especially want to mention a few people by name;

Since their support had such a crucial role in my endeavor, I would like to thank my promotors and co-promotors: Professor Bert Joosten PhD, Professor Maarten van Kleef MD PhD, Jaap Patijn MD $\mathrm{PhD}$, and Ronald Deumens PhD, the Principal Investigator of the laboratory of neuroregeneration at the University of California - San Diego: Professor Martin Marsala MD, the chief scientific officer of Neuralstem Inc.: Karl Johe PhD, and my wife/colleague/secretary: Marjolein Leerink MPhil.

Also, I would like to mention my direct day-to-day colleagues for their outstanding support, their more than perfect amount of humor, and their blindingly bright perspectives: Alex Platoshyn, Andreas Glud, Camila Santucci, Danielle Goldberg, Dasa Dolezalova, Jan Strnadel, Jose Corleto, Liesbeth Knaepen, Marian Hruska-Plochan, Michiel Truin, Mike Hefferan, Osamu Kakinohana, Rachel Slangen, Robby Jaken, Sabien van Neerven, Sandee Nguyen, Silvia Marsala, Wiel Honig, Wouter Pluijms, and my allways-there-coffee-colleague: Pieter Hoogland.

And, last but not least, I would like to give very special thanks to my parents: Joost and Anita.

Furthermore, I would like to give thanks to the facilitating support of the Maastricht University Medical Centert, and in particular the department of Anesthesiology and the department of Neuroscience. 


\section{Colofon}

\section{Uitgeverij BoxPress}

Printed by: Proefschriftmaken.nl || Uitgeverij BOXPress

Published by: Uitgeverij BOXPress, 's-Hertogenbosch

Published in 2014

ISBN: 978-90-8891-828-5 